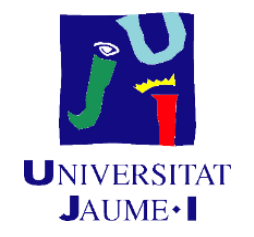

\title{
The effect of family structure and family support on Women's Coping with fertility treatments
}

\author{
Dissertation for Doctoral Degree \\ Presented by: \\ Limor Rimer
}

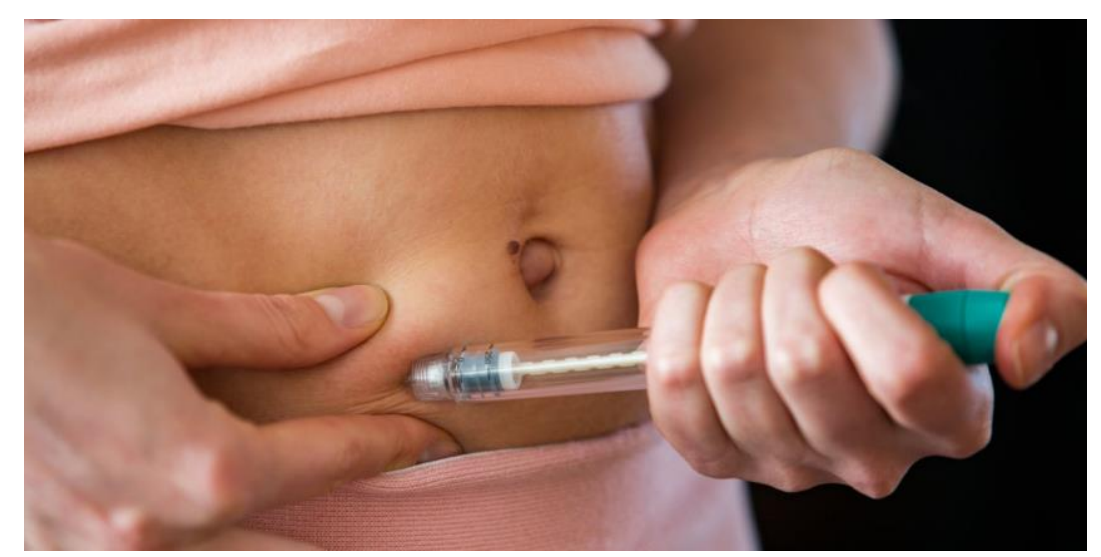

Under the supervision of: Dr. Adelaida Zabalegui Yárnoz

July 2020 


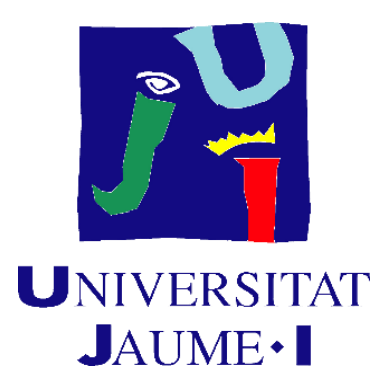

Doctoral Programme in Nursing science

Universitat Jaume I Doctoral School

\section{The effect of family structure and family support on Women's Coping with fertility treatments}

Report submitted by: Limor Rimer in order to be eligible for a doctoral degree awarded by the Universitat Jaume I
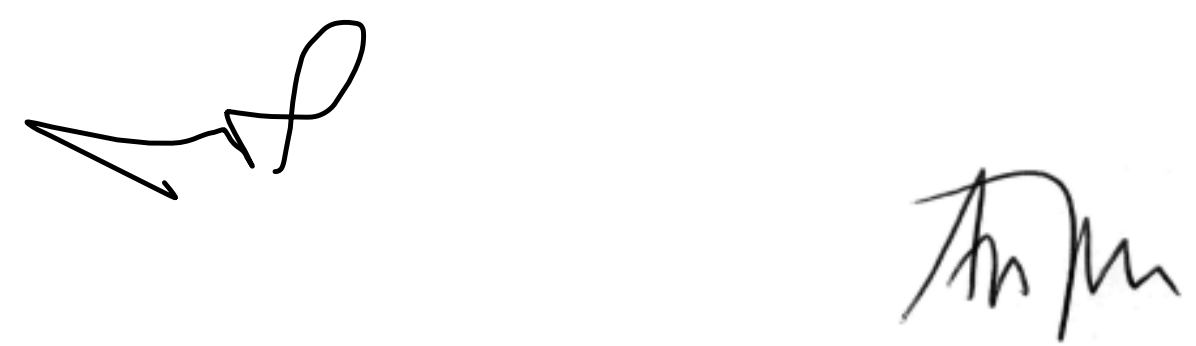
A son, if only I had one"

A small and clever boy

With dark curls to hold his hand

And to slowly walk along the garden paths

A small boy"

(Rachel Bluwstein's poetry) 


\section{Acknowledgments}

As dissertation's writing comes to its end, I would like to thank many persons who were important to me during the whole process.

Firstly, I extend much gratitude to those who participated in this study and contributed to its success. Thanks for volunteering and kindly sharing your experiences.

To my beloved son- Almog, Thank you for your support and encouragement throughout the process. For inspiring me as well as believing in me. You share this achievement!

I would like to express my most sincere gratitude to Dr. Adelaida Zabalegui my supervisor for your knowledge, your professional academic accompaniment and support throughout the whole period and for pointing out details as well as the big picture.

My sincere thanks also to Dr. Ray Silverman my advisor, for your guidance, patience, and assistance at every stage. Thank you for always being there for me.

Also, I would like to thank Dr. Yfat Viner MD, the clinic's director, for your assistance in applying for the ethics committee, and to Dr. Amir Ravhon MD the fertility specialist, for your professional consultancy, your wise advises and encouragement.

I also would like to thank my colleagues who assisted with locating and recruiting participants for this study.

Special appreciation to Zeev Minkin who was responsible for the doctoral administrations throughout the whole process. Thanks for your assistance in achieving my dream.

\section{And finally,}

I would like to dedicate this dissertation in memory of Dr.Tal Dror my advisor, who kindly accompanied me about halfway and unfortunately succumbed to cancer. 


\section{LIST OF SCIENTIFIC PAPERS}

1. Rimer, L. (2019). The Effect of Family Structure and Family Support on Women's Coping with Fertility Treatments - A Systematic Review. International Journal of Nursing, 6(2), pp. 102-111. DOI: 10.15640/ijn.v6n2a11. URL: http://dx.doi.org/10.15640/ijn.v6n2a11 ISSN- 2373-7662 (print), 2373-7670 (electronic version).

2. Rimer, L. (2020). The Effect of Family Structure and Family Support on Women's Coping with Fertility Treatments. International Journal of Nursing, 7(1), pp. 18-29.

DOI: 10.15640/ijn.v7n1a3. URL: http://dx.doi.org/10.15640/ijn.v7n1a3 ISSN- 2373-7662 (print), 2373-7670 (electronic version).

3. Rimer, L. (2020). Fertility treatments among single mothers by choice in Israel, their experience and support systems. International Journal of Advance Research in Nursing, 3 (2), pp. $48-52$.

DOI: $10.33545 /$ nursing.2020.v3.i2.A.100.

URL : http://www.nursingjournal.net/article/view/100/3-2-10

ISSN: 2617-9806 (print), 2617-9814 (electronic version).

Publication in Hebrew- Rimer, L. (2020). The Effect of Family Structure and Family Support on Women's Coping with Fertility Treatments: A Cross-Sectional study. The Israeli Nurses Association Magazine, pp. 38-42. 


\section{Table of contents}

Acknowledgments ........................................................................... II

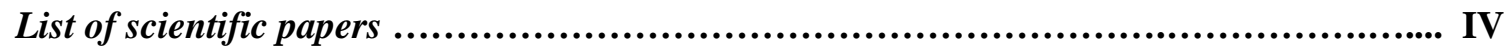

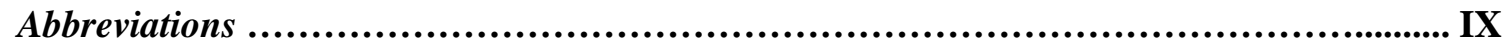

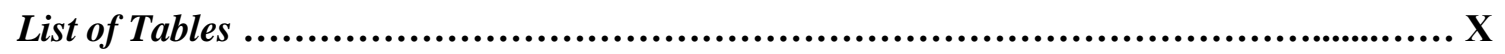

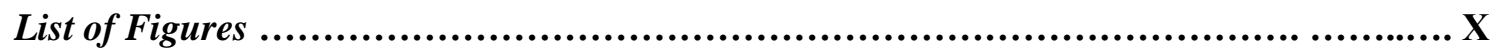

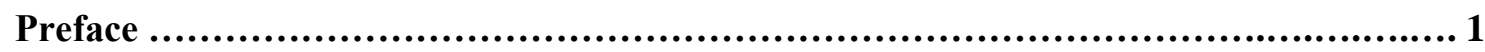

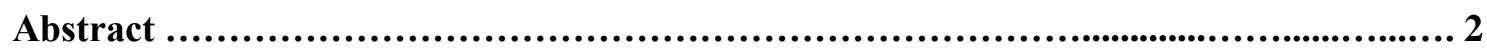

Chapter 1: Introduction ........................................................................... 4

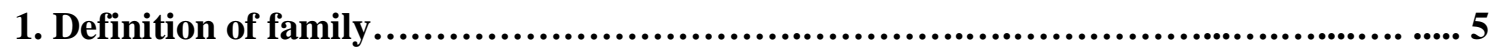

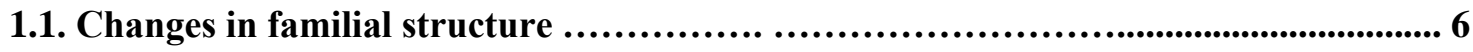

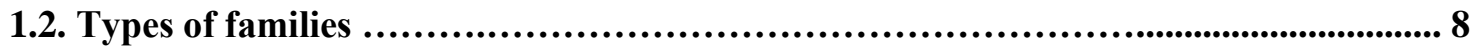

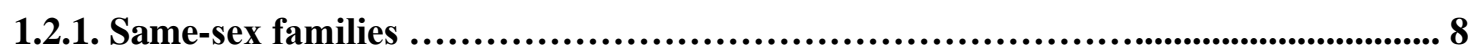

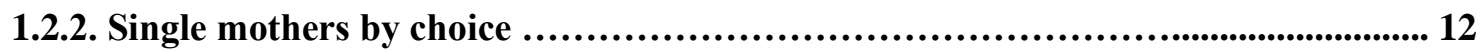

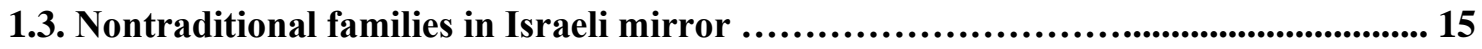

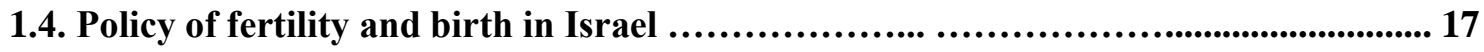

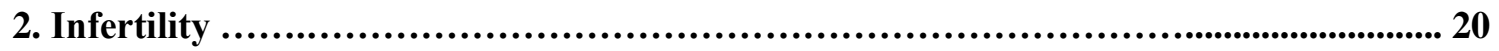

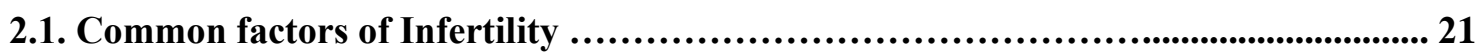

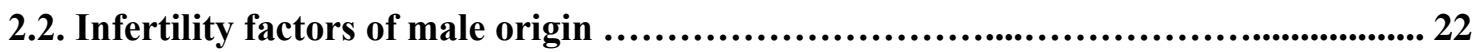

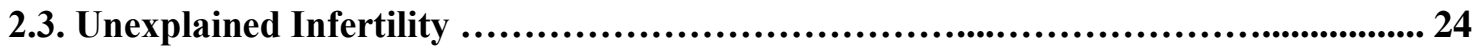

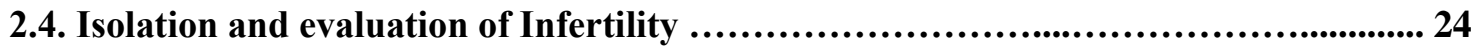

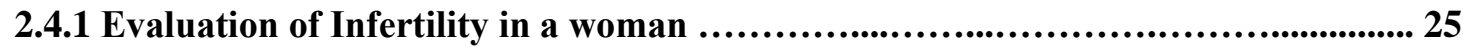

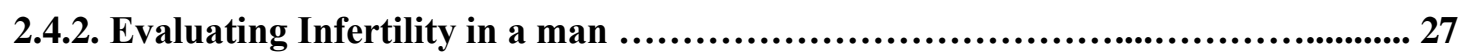

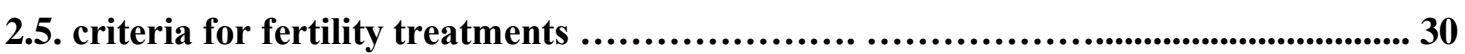

2.5.1. Treating Infertility of female source .................................................... 32

2.5.2. Infertility treatment of male origin ........................................................ 35

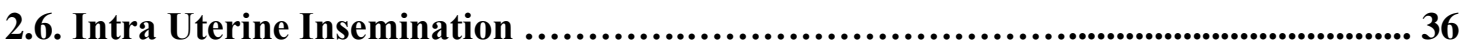




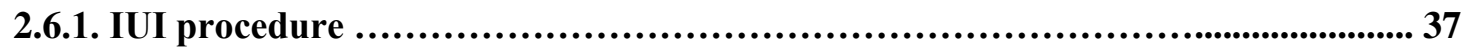

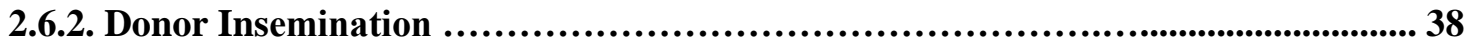

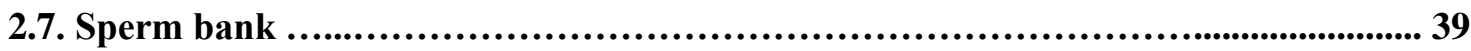

2.7.1. Bill of sperm donation regularization in Israel ............................................ 40

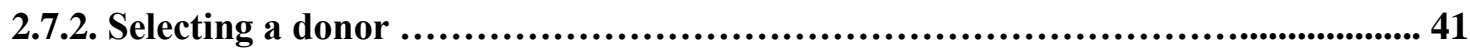

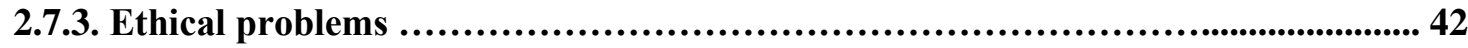

2.8. Number of treatments cycle before transition to IVF ......................................... 43

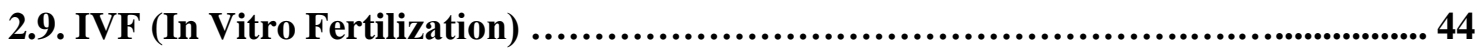

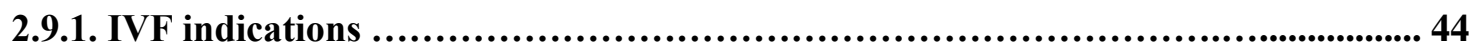

2.9.2. Advantages and shortcomings of IVF treatments ................................... 46

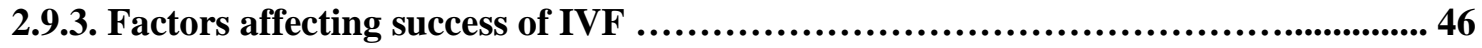

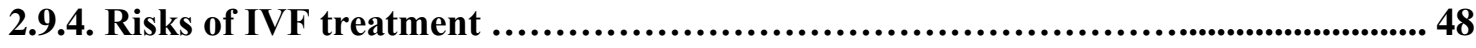

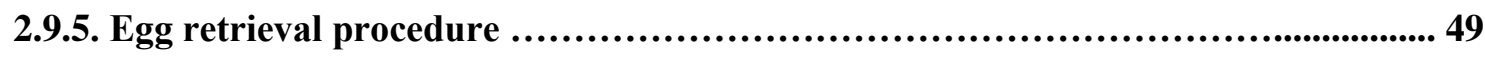

2.9.6. Cryopreserved embryo transfer ......................................................... 49

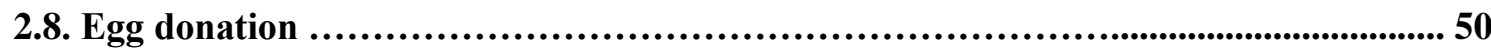

3. Fertility difficulties, A psychological and emotional aspect ....................................... 51

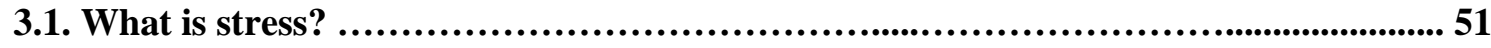

3.2. Infertility and treatment as stress factors ..................................................... 51

3.3. Emotional distress of fertility treatments ..................................................5 52

3.4. Effect of fertility treatments upon intimacy ...............................................55

3.4.1. Emotional distress amongst women .................................................... 56

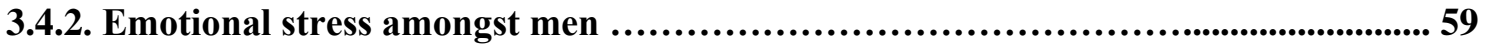

3.4.3. Differences between men and women ...............................................60

4. Coping with fertility treatments of women ...................................................... 62

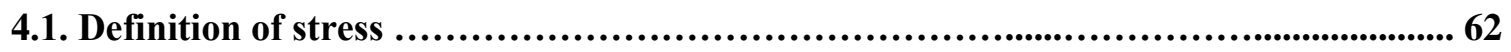

4.2. Transactional model of stress and coping .................................................63

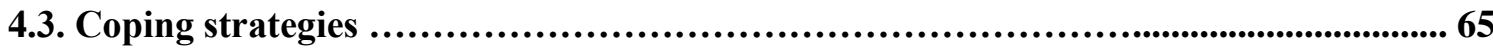

4.3.1. Coping with infertility and fertility treatments ............................................ 68 


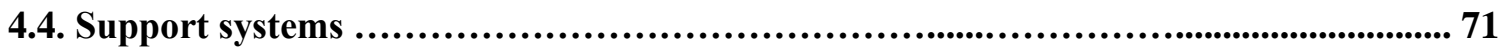

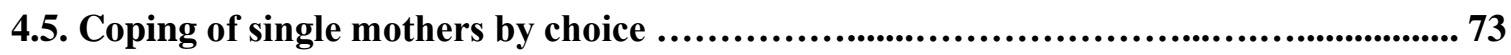

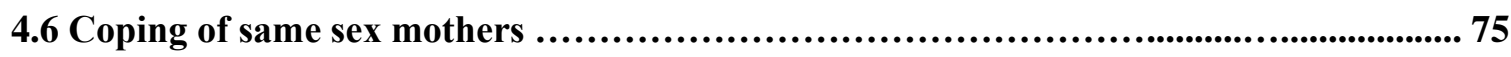

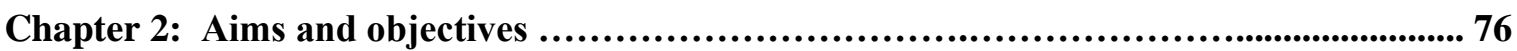

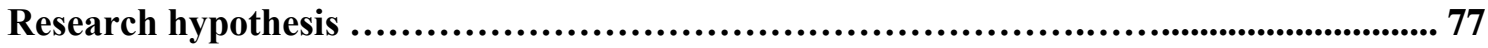

Chapter 3: Methodology ........................................................................... 77

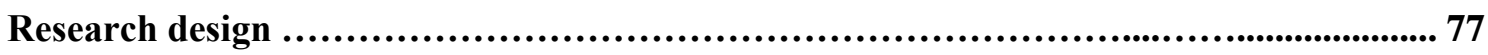

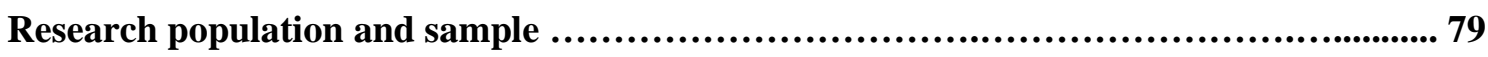

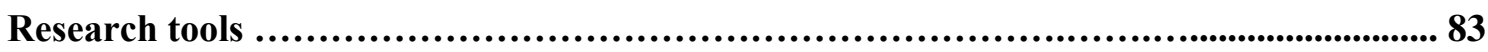

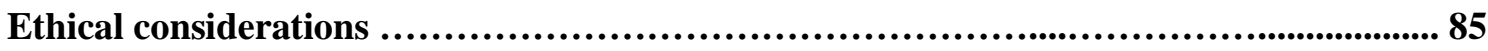

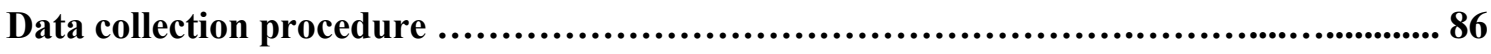

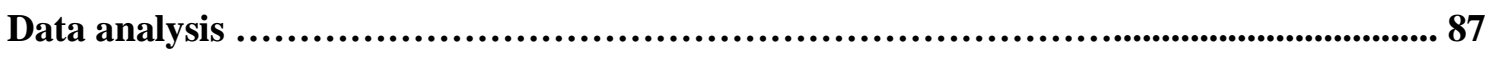

Chapter 4: Findings ............................................................................... 88

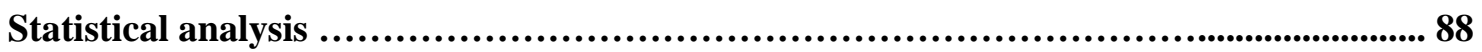

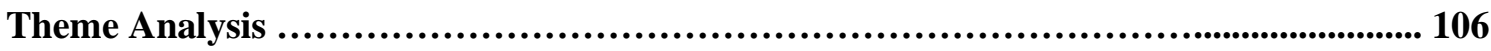

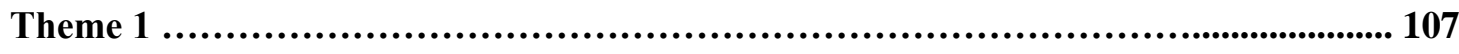

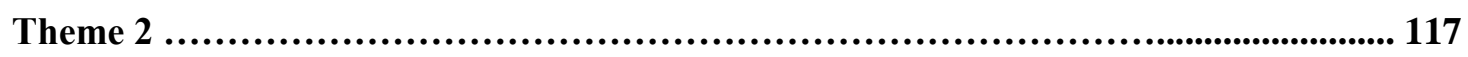

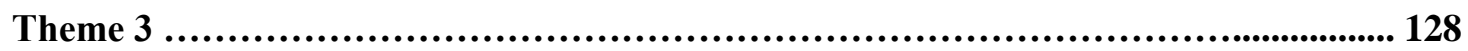

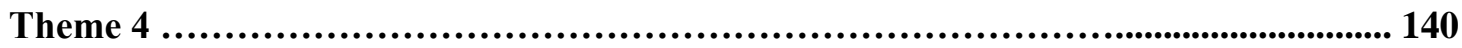

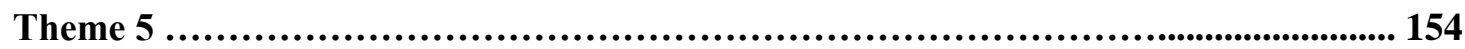

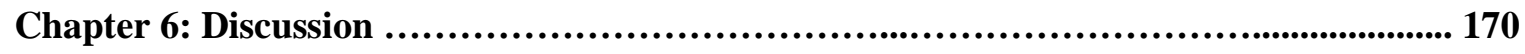

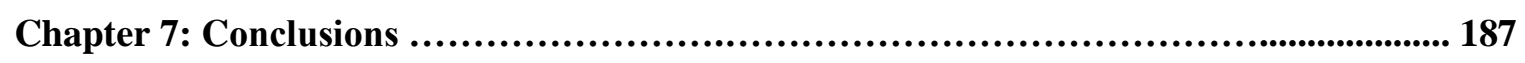

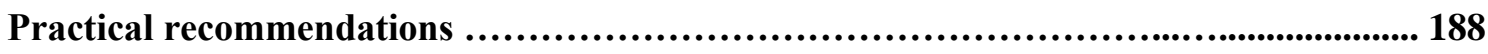

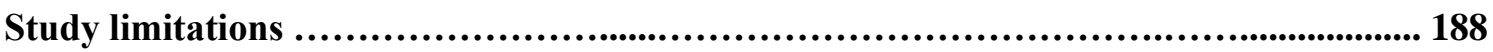

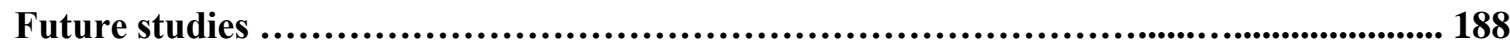

Chapter 7: References ............................................................................ 190

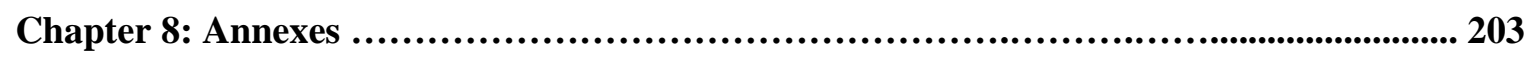




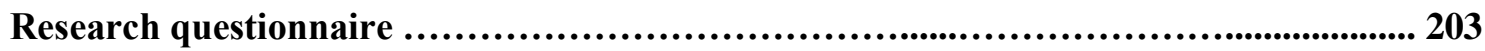

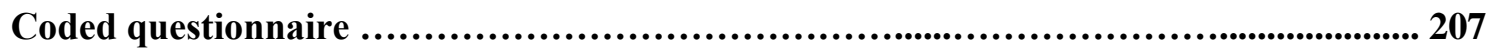

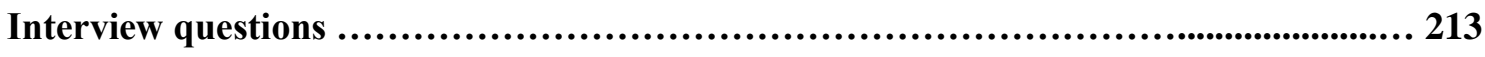

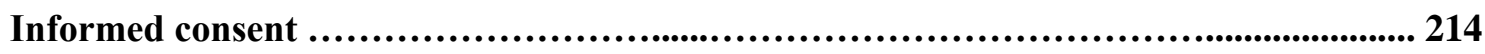

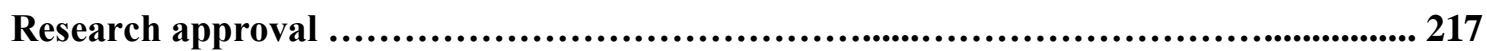




\begin{tabular}{|c|c|}
\hline AMH & Anti Mullerian Hormone \\
\hline ART & Assisted Reproductive Technology \\
\hline ASRM & American Society for Reproductive Medicine \\
\hline BMI & Body Mass Index \\
\hline $\mathbf{C F}$ & Cystic Fibrosis \\
\hline CMV & Cytomegalovirus \\
\hline $\mathrm{COH}$ & Controlled Ovarian Hyperstimulation \\
\hline $\cos$ & Controlled Ovarian Stimulation \\
\hline E2 & Estradiol \\
\hline ECG & Electrocardiography \\
\hline EMF & Electromagnetic Field \\
\hline FSH & Follicle Stimulating Hormone \\
\hline GnRh & Gonadotropin Releasing Hormone \\
\hline HCG & Human Chorionic Gonadotropin \\
\hline HIV & Human Immunodeficiency Virus \\
\hline HMG & Human Menopausal Gonadotropin \\
\hline HMO & Health Maintenance Organization \\
\hline HCG & Hysterosalpingography \\
\hline ICSI & Intracytoplasmic Sperm Injection \\
\hline IUI & Intra Uterine Insemination \\
\hline IVF & In Vitro Fertilization \\
\hline $\mathbf{L H}$ & Luteinizing Hormone \\
\hline MNC & Modified Natural Cycle \\
\hline NIS & New Israeli Shekel \\
\hline OHSS & Ovarian Hyperstimulation Syndrome \\
\hline OTA & Oligo- Terato Asthenospermia \\
\hline PCOs & Polycystic Ovary Syndrome \\
\hline PCT & Post Coital Test \\
\hline PID & Pelvic Inflammatory Disease \\
\hline PGD & Pre Implantation Genetic Diagnosis \\
\hline SART & Society for Assisted Reproductive Technology \\
\hline STD & Sex Transmitted Disease \\
\hline TESA & Testicular Sperm Aspiration \\
\hline TESE & Testicular Sperm Extraction \\
\hline WHO & World Health Organization \\
\hline
\end{tabular}




\section{List of Tables}

Table1- Quantitative part: descriptive statistics of the demographic variables ......................... 80

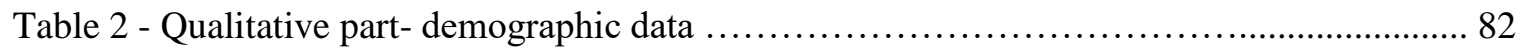

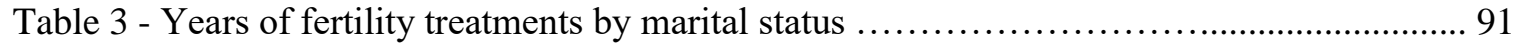

Table 4 - Actions during trying to get pregnant by marital status ......................................... 92

Table 5 - Testing following attempts to get pregnant by marital status .............................. 92

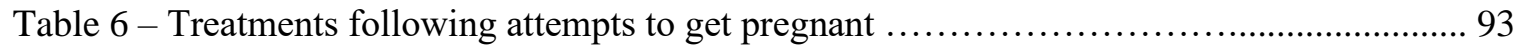

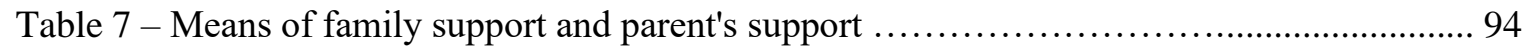

Table 8 - Correlations with family and partner support ............................................ 95

Table 9 - Mental treatments following attempts to get pregnant ................................. 95

Table 10 - Feelings following attempts to get pregnant by marital status .......................... 96

Table 11 - Sharing feelings with other people by marital status .................................... 96

Table 12 - The effect of reproductive treatments on partner's involvement and relationship........ 97 satisfaction.

Table 13 - Coping strategies as a couple following failure of reproductive treatments ........... 98

Table 14 - Sharing feelings with other people by marital status ................................... 99

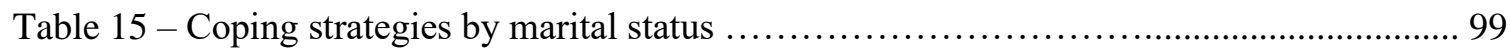

Table 16- Differences in effects of treatments by marital status ................................. 100

Table 17 - Differences in effects by type of treatment ................................... 101

Table 18 - Differences in coping strategies by type of treatment .............................. 103

Table 19 - Differences in effects of treatments by children .................................. 104

Table 20- Differences between women with and without children in coping strategy ............. 105

Table 21- Correlations between emotionally coping strategies and age ....................... 105

\section{List of Figures}

Figure 1 - Transactional model of stress and coping of Richard Lazarus ................................ 64

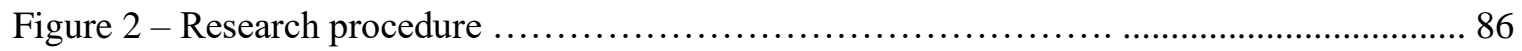

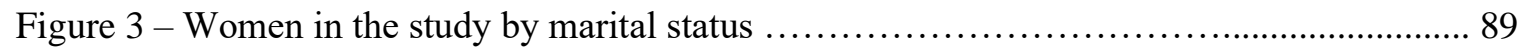

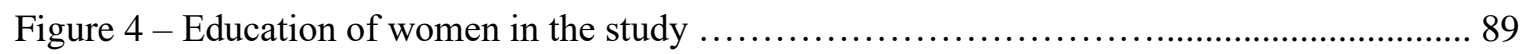

Figure 5 - Education of women's spouse in the study ........................................................... 90

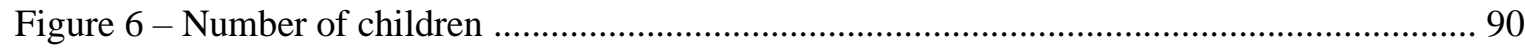

Figure 7 - Common coping strategy of couples following failure of reproductive treatments .... 98 
Figure 8 - Differences in effects of treatments by marital status .................................. 101

Figure 9 - Differences in effects of treatments by type of treatments ............................ 103

Figure 10 - Differences in effects of treatments by children ........................................ 104

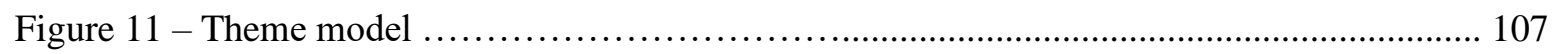




\section{PREFACE}

The role of the nurse in fertility treatments and IVF is continuing to evolve at a fast pace, presenting a variety of challenges and opportunities. Traditional nursing scenarios involved clinic management, supervision of patients and data collection. With the increase in requirements for fertility treatments, the nurse is involved in all kind of aspects of the treatment. nowadays, the main role of the fertility nurse is to obtain the desire, strength, and knowledge the patients need in order to cope with the psychological situation caused by the treatments. Nurse is also the main healthcare personal with whom the patients are in touch throughout the treatment. Following twelve years as a fertility nurse, I got involved in every aspect of the treatment starting with the diagnosis, gained expertise in the medical protocol, the routine follow-up and provided the required information and above all, provided emotional support through the ups and downs accompanied the treatments.. During my years of experience, I got to know each and every patient individually and fostered a relationship with each patient, this close acquaintance assists in evaluating their individual needs with a holistic perspective, so I was able to identify problems, provide their care differently and provide support and encouraging in order to achieve a successful pregnancy.

This thesis results in light of the crucial role we have that connects the patients to the dream of becoming parents, the importance of guiding throughout fertility treatments, help navigate them through the process and preventing the crisis resulting from the exhausting and frustrating process. 


\section{ABSTRACT}

Introduction: The use of innovative fertility treatments contributed to the creation of new family structures that did not exist before, such as single-sex families and single-parent families. In the past, the only acceptable way to have a child was within marriage, nowadays fertility treatments solve various fertility problems and are technologically advanced and effective, yet many report repercussions for other areas of life, such as marital and social relations.

Aim: To examine three main family structures: married couple, single mother and same-sex couple, as well as the coping of women undergoing fertility treatments, the difficulties they experience during treatment and their support systems.

Materials and Methods: The study employed a mixed methodology, in which quantitative and qualitative data were gathered simultaneously to explore women's difficulties while undergoing fertility treatments and their support systems. a questionnaire created for the purpose that evaluates coping with the fertility treatments and their support systems if they exist. The questionnaire based on the literature review was distributed to 450 women who were undergoing fertility treatments in Israel. tracing and recruiting the participants was accomplished through sampling layers which were determined according to the research variable that corresponded with the population that was represented in the sample. All the data in the questionnaires were recorded in an Excel table according to the encoded questionnaire and analyzed with SPSS software. in addition, in phase II based on the phenomenological design interviews to 15 patients overall. Data collection was quota sample - three clusters were selected and in each cluster a convenience sample was conducted until an affixed quota was filled. A qualitative content analysis approach (Graneheim \& Lundman, 2004) was used to analyze the interview transcripts. Each interview was tape recorded, read multiple times to grasp the entirety of the data collected and transcribed verbatim. the written text was validated against the sound recordings. The transcribed text was divided into meaning units depending on content, the meaning units were then divided into categories and subcategories by scrutinizing their similarities and differences, finally Then 5 central themes were created.

Results: for the quantitative part 450 women participated in this study. Sample was divided into three groups $-33.3 \%$ were married, $33.3 \%$ were single and $33.3 \%$ had a female partner with average age of 36.41 . Results show that single women conducted more treatments $(73.3 \%)$ 
in comparison with married women (65.3\%) and women with female partner (54.7\%). women with female partner reported higher partners' support in comparison with married women (F $(1,298)=121.57, \mathrm{p}<.01)$. Women who have no children are more negatively influenced in comparison with women with children, Finally, younger women have more support, but they also more negatively affected from fertility treatments in comparison with older women. in the qualitative study 15 interviews were conducted ( 5 of each family structure), mean age of women was of 36. The qualitative part yield 5 main themes: Attempts to conceive and the attendant difficulties, response to finding out about the problem, choosing an appropriate solution, and beginning treatment, coping with the outcomes of the chosen treatment and being in treatment. Conclusions: family structure affects women's coping with fertility treatments. Married women reported more emotional support from their family than single women and women with female partner, hence, they reported fewer negative emotions and more hope regarding the treatments. On the other hand, results showed that women with female partner reported higher partners' support in comparison with their married counterparts. 


\section{Chapter 1: Introduction}

The twentieth century has seen many revolutions; none with more impact on the family in the industrialized world than the changes to the traditional familial framework in the last third of the last century Marriage age has increased, and the percentage of men and women who never marry has as well. No less important is that family has decreased, and instead became increasingly relevant families of much more diverse structures compared to the past (Bar-Sela 2003).

The use of innovative fertility treatments has contributed to the emergence of new family structures such as single-sex families and single-parent families. In the past, the only acceptable way to have a child was within the marital framework, whereas today there is an upward trend among single women and lesbians to try getting pregnant with the help of medical assistance. (Hashiloni- Dolev,2013).

All fertility patients are affected emotionally: couples with difficulties conceiving, as well as single women or lesbians who choose this method as a way of bringing children into the world. People struggling with fertility problems and treatments are usually young and healthy (apart from their difficulty conceiving), but the treatment of this condition requires medical interventions that can have the same emotional impact as a severe chronic disease. The grief and loss felt by individuals or couples that might accompany fertility difficulties is often exacerbated by the fact that it is suffered in private and not socially recognized or because sometimes the couple or individual keep their experiences to themselves, thus contributing to their sense isolation and distress (Kornik, 2012).

Though fertility treatments offer the prospect of parenthood to many more than ever before, they also involve complex mental coping: they are demanding, complicated, and cumbersome to carry out, as well as damaging to the self-image, spousal relations, and the very fabric of one's personal life. This crisis is referred to in the literature as the reproductive trauma (Schwerdtfeger \& Shreffler 2009).

The literature acknowledges the difficulties resulted from fertility treatments. A significant amount of research started emerging describing the psychological effects and distress due to fertility treatments among heterosexual couples, their way of coping, gender-based outcomes, and associated marital and sexual consequences. However, very little is known about the difficulties, way of coping and psychological effects among single mothers and lesbians undergoing fertility treatments. This current study examined the traditional family unit (married 
couples) compared to lesbian couples and single mothers in their way of coping during fertility treatments as well as their support systems.

\section{Definition of family}

Family is the most basic unit in society and therefore, it is essential for the development and maintenance thereof. It is hard to imagine someone who never experienced being part of a family. Despite the historic centrality of a nuclear family unit (a mother, a father and a child) there are many definitions to family. According to Census Bureau (2000), a family can be a group of people being together by virtue of marriage, birth or adoption or by mutual residence or close emotional ties. Families can include people who claim that they are descendant of mutual fathers in a dynasty or a tribe. The family system is a basic unit of society which evolved with changes in needs and demands of individuals and society, family was a means for development of cultures and ideas (Jade et al., 2007).

Bails and Parsons begin with defining the functions of a family, whereas the first is the socialization process, through which it is possible to learn and generalize between the social system, personality and culture. Human beings coexist due to a moral consensus and family has a central role in endowing social order, this consensus is built on. The second function of family is its being a skilled agent of segregation and specialization. Family according to Parsons is "neolocal" - living in its own house, separate from the spouses' parents, an independent financial unit and the division of roles in the house is based upon gender and age. Division according to age is biological, so an inevitable datum is the dependence of a child on his parents and inequality is expressed in authority relations and in the duty of children to obey their parents. However, division according to gender is not only biological however social and functional for society as well, as a nuclear family that has specialization is more stable.

Hence, there is great importance to gender roles division in a family. A woman fills the expressive-emotional role while a man fills the instrumental-task role. As family and the employment system are dependent upon one another, there is a need for specialization of each spouse in the different roles suitable for them. This approach recognizes only a nuclear, white, middle-class heterosexual family, and does not legitimize different forms of families and different cultures, hence, it ignores social differentiations. Additionally, theoreticians ignore balance of power and conflicts within a family as well as historical processes. Meaning - they 
assume a sequence of developments, however, there is no relation to specific historical processes.

Other definitions of family tend to be influenced by social factors. Family definitions which include joint residence and local partnerships can be considered as partially grey areas, however, they are more acceptable socially and those families often receive legal rights of "family" (Hanson \& Lynch, 2013).

In summary, the task of defining a family is not easy and the difficulty stems from the mythology surrounding the idea of a family (Aulette, 2010).

\subsection{Changes in familial structure}

Many a revolutionary change has occurred in the twentieth century, however, the dominant revolution in the last third of the century, as related to the industrialized world, is revolution of a family. In recent 35 years, several processes have taken place simultaneously, integrated with one another. Birth in Western countries reduced to a negative procreation balance of entire industrialized world. Women are much more included in employment, in higher education, in professions, in policy, in art; and in comparison, even in diseases considered "male" such as heart diseases. Matrimonial age has increased, and rate of men and women who are not getting married at all has increased. And finally, as important: the stability of family has lessened. Every second or third family in the industrialized West is expected to a divorce. In Israel, Japan, Italy and Spain the rates are slightly lower, however, the rate of divorce has increased in them as well. Even amongst those living in couples, many renounces a formal marriage ceremony and some of the privileges and duties involved in marriage (Bar Sela, 2002).

Increasingly, both women and men wish to first establish themselves in labor market before funding a family and the decline of marriage rates is related to the emergence of more nontraditional family forms such as cohabiting, single women and same-sex families (OECD 2011).

New familial patterns and increased instability creates complex familial and financial connections spanning across a great number of households: the unstable life arrangements and complex familial relations affect as well, the health and welfare of children and adults and create challenges for both families and policy makers. As compared to the 50's, men and women have more socially acceptable decisions regarding how, when and with whom to create proximity relations and in what context to have children. Marriages, shared residence, and parenting are no longer always go together and marriage is as well not necessarily perceived as commitment for lifetime. As families become more complex, the relations between family members become 
complex as well. In complex families, there is a variety of biological, social and familial relations amongst family members and within home (such as stepfamilies and relations of halfbrothers). Definition and estimation of such families is challenging, as each family member cannot "consider" or present his relations with all other of family members in same way (Venaman \& Scomegna, 2016).

In time of change from single dominant model of family order to a legitimate multiplicity of models, family weakens, gets undermined and the tensions within it increase. There are multiple tension aspects in a family. One witnesses severe familial phenomena of poverty, violence, neglect and abuse. There is an increase in families exhibiting distress and pain and dissatisfaction with life quality in the family. Distress of a family stems in most cases, from lack of resources to reshape the signs of order. It as well stems from the need to turn a big portion of family's energies to the task of shaping order while fulfilling its instrumental and emotional tasks (Stryar, 2009).

Peterson (2002) maintained that with all these changes in individuals, chronic changes within a family and changes in society, familial stability might be undermined. In order to become more flexible, families need to achieve balance. They can do that by achieving stability and control over the following four protection mechanisms: familial consolidation, familial flexibility, familial communication and familial meaning. Consolidation relates to the extent of a family's emotional attachment and dependency on one another. Flexibility relates to the extent a family can change its patterns upon occurrence of an event. Familial communication describes the amount and methods in which a family talks freely and expresses emotions towards one another and the term of familial meaning relates to capabilities of families to identify their identity and their worldview when they discover solutions or manage a challenge in their lives. Composition of a family influences the choice of the family's strategies. Families headed by a single parent often develop different strategies than those of a household headed by two parents. The lesbian family system might as well develop different strategies than those of a heterosexual familial system. The composition of a family affects family dynamic by shaping the strategies activated as part of the system's effort to execute the tasks. Determining familial functioning is anchored in culture. Strategies accepted by society become standard according to which effectivity is being judged. When strategies taken by a family are in one line with those accepted by society, the family is judged as effective whereas when strategies taken by a family deviate slightly from familial norms, the family has higher chance to be judged as noneffective (Aulette, 2010). 
Rice and Degenova (2002) indicate changes in the way people see family. They testify that currently; family fills a bigger role of meeting personal needs such as emotional confidence rather than only filling the traditional role of a family as an institution responding to needs of society.

\subsection{Types of families}

A common perception of traditional family turned in recent years into an array of diverse family structures. Two prominent structures in this array are families headed by lesbian mothers and heterosexual single mothers by choice. Those family structures take their position alongside with more traditional types of families and as a result, these mothers face new adaptation challenges (Cabc 2010)

\subsubsection{Same-sex families}

A same-sex family is composed of two people of same sex, having a permanent intimacy connection. As a familial structure, a family of lesbian two mothers-parents is not a new structure, however, the way in which lesbian families are created already develops. Increase in public recognition (increase of acceptance of parenting of same sex) and extended legal recognition of relations of people of same sex, have reshaped the social view, in which homosexuals and lesbians create a romantic partnership. The number of married couples of same sexes rapidly increases, following the decision of United States Supreme Court in June 2015, to permit same-sex marriage in every State in the United States. In October 2015, it was estimated that same-sex marriages constitute $45 \%$ of all same-sex couples (Gates et al., 2015). California was the first state which legislated throughout the country the recognition of samesex couples when it created its local partnership registration in 1999. The local partnership offered same-sex couples some of the benefits usually related with marriage, meaning, sick visitation rights and the ability to be registered as heirs in a will of the spouse. Massachusetts has become the first State allowing marriage of same-sex couples in 2004 (Gates, 2015).

Sexual minorities structure a family in variety of ways:

- In an atmosphere of social stigma, many a lesbian mother, conceived her children through heterosexual relationships or marriage before she had acknowledged her lesbian identity. In recent years, lesbians gained greater access to fertility treatments and more chances to build their families without a direct involvement of male spouses.

- Some of them enter same-sex relations after their children were born or adopted. 
- Others become parents in a context of same-sex commitment, these families are often called: intentional or planned same-sex families, a phenomenon that has grown amongst other things due to advancement in fertility technology.

Out of all above mentioned family types, a stepparent in same-sex family arrangements, might represent the dominant familial structure (Goldberg et al., 2014).

Probably, a number of factors have led to this change. First and foremost is the fact that positions towards sexual minorities have changed, which leads to a greater public acceptance of lesbian families, public policy changed accordingly. For example, in England, the phrase "a child's need for a father" was deleted from clause 13(5) of The Human Fertilization and Embryology Act - 2008. Similar changes were recommended previously by the American Society for Reproductive Medicine's Ethics Committee in 2006 (Scheib \& Hastings, 2012).

There are evidences implying that marriage has a stabilizing effect upon same-sex couples, as lesbians have a desire to be married and have views regarding the purpose of marriage that are similar to views of the general population. A survey held by Pew Research Center in 2013, numbered the reasons to get married, the answers same-sex couples gave were not different than those of the general population in their belief that love, friendship as well as commitment for lifetime were the three most important reasons to get married. The only significant difference between same-sex couples and the general population in this context was that samesexual couples assigned greater importance to the rights of legal benefits as a reason to get married than the general population. This might not be a surprising difference, as the significant media attention focused on rights and legal benefits that were not available to same-sexual couples in those places where they were not able to get married.

In comparison to 20 years ago, same-sex couples live in shared residence and they separate and divorce in similar rates as those of heterosexual couples. In March 2015, Gallup estimated that approximately $40 \%$ of same-sex couples were married. Around the world, marriage or any other form of legal recognition through civil partnerships or registrations are now available for samesex couples in Northern, Western and Eastern Europe, big parts of North and South America and in South Africa, Australia and New Zealand. Opposite to that, homosexuality is still considered to be criminal, and in some cases with death punishment, in all of Africa, Middle East, South-East Asia and in Russia and in many countries in the South Pacific and the Caribbean (Gates, 2015). 
Same-sex couples usually choose one of several courses for pregnancy: sperm donation, adoption, or surrogacy. It is no surprise that those populations constitute presently most of the clients of sperm donation in United States. The end result is that presently, there are more families headed by lesbians that raise children who conceived through sperm donation than ever before (Scheib \& Hastings, 202).

Reproduction possibility for same-sex couples requires an intervention of a third party from the other sex in order to provide gametes. Medical treatment spans from a relative routine to a more complex, however, the process is always involved with making difficult decisions, legal considerations, psychological demands, and substantial expenses. The journey for parenthood can often be challenging for lesbian women who might encounter barriers to treatment such as: prohibitive expense, homophobia, and geographic location (Greenfeld \& Seli, 2016).

In recent years, social change as well as an increasingly growing presence of lesbian mothers, assists to a greater awareness and sensitivity on the part of professionals in field of healthcare who encounter a sexual minority requesting to get pregnant through sperm donation. Although reports regarding insensitive treatment by such professionals continue to appear in literature, for example, women from a sexual minority continue to encounter clinics which are not "suitable" for these patients (Goldberg et al., 2014).

How does women from a sexual minority experience and cope with failed treatment attempts is a subject that has been scarcely researched. Despite a study that found that women from a sexual minority on average would invest less in bringing a biological offspring and might turn to adoption as a means to parenting. In fact, same-sex couples are more expected to adopt than different sex couples (Gates, 2013).

A research by Goldberg et al. (2009) reveals that lesbian women in average move with greater ease from an attempt to conceive to adoption as compared with heterosexual women, however, there is still a considerable number of lesbians who are still struggle with a passion to give birth to biological children, they have greater desire to justify their roles as parents and are less concerned with traditional purposes of raising children than heterosexual parents.

While society has changed in its views towards same-sex couples and their family members, it had changed as well in its positions towards gender and the norms in which men and women organize their relations and their families. Changing social norms that relate to gender and parenthood might fulfill a role in explaining the decisions regarding how to divide time between work and family, there is evidence to the fact that same-sex couples deliberately prefer a more 
equalitarian division of work particularly as rejection of traditional roles of men/women in parenthood and in comparison to heterosexual fathers, all lesbian mothers were more emotionally involved, supportive and respectful of children's autonomy and less belligerent (Scheib \& Hastings, 2012).

Gates (2013), for example, demonstrated that most children raised by same-sex couples are identified as biological children of the house owner or as stepchildren of the house owner, in case of the last, these children are probably biologically related to the female spouse.

Same-sex families are exposed to alienation and lack of support from their origin families. They occasionally experience persecution, disinheritance of rights and invalidation thereof. These families cope as well with social stigma, nonrecognition and support on a legal plain, as there is a legal difficulty to institutionalize the same-sex intimacy relations and there is a difficulty to recognize joint parenting upon absence of blood relation (Goldberg, 2010).

Until 1975, same-sex sexual tendency was included in the psychiatric disturbances manual (the DSM - Diagnostic and Statistical Manual) and was defined as being psychopathological and was considered as a criminal perversion. Psychiatrists defined Lesbianism as a type of neurosis which includes narcissistic pleasure and lack of sexual maturity. However, in 1973, the American Psychiatric Association removed homosexuality as a psychopathological syndrome from the DSM (Greenfield \& Seli, 2016).

A large portion of legal and political debate of recent decade regarding awarding of a possibility for same-sex couples to get married, focused on the adjustment of those couples as parents. After a meticulous examination of evidences presented by researchers from both sides of the problem, it was found that same-sex couples are as good parents as their heterosexual peers. All differences in welfare of children who were raised in same-sex families and in heterosexual families might be explained not by the gender composition of their parents, but rather due to the fact that children who were raised by same-sex couples experienced in average a greater familial instability, as most of those children were born to heterosexual parents one of which being presently engaged in same-sex relations. This pattern currently changes in light of a reduced social stigma following which more same-sex people come out of the closet in earlier stage in their lives and thus tend less than their peers in the past to give birth to children in a frame of heterosexual relations, but rather tend to have children who were born as part of samesex family, being in a stable relationship (Gates, 2015). 


\subsubsection{Single-mothers by choice}

In the past, single-parent families stemmed from death of a spouse, collapse of marriage or births by unmarried women. Presently, financially, socially and politically, most women can operate consciously and decide whether to reproduce, when to reproduce and how to reproduce. A combination of a change of social moral and technological advancement enabled the appearance of single-parent family formation by choice (Van Kreiken et al., 2014).

In recent years, there is an increase in number of children born to unmarried single-parent mothers as a result of unplanned pregnancies. However, the most recent type of single-parent family is of single women becoming mothers intentionally without a partner, usually called solo mothers, or single-parents by choice. Single mothers currently constitute about $19 \%$ of all households with children, this number is expected to grow in light of a demographic change of first-time mothers in older age. In the European Union, in majority of cases, the phenomenon relates to women (Golombok et al., 2016).

Single mothers by choice, are mainly heterosexual women in their late 30 's and 40 's, educated and financially independent, who can achieve motherhood through various courses, however they often do that through treatment in fertility clinics for treatment from sperm donation. However, the possibility that single women have access to fertility treatments aroused extensive public and political debate, conservative parliament member Lain Duncan Smith described the bill as "the last nail in the coffin of traditional family life and an additional strike for fatherhood, as his departure from the nuclear family, the ethics of the choice of going for motherhood without a male spouse is doubtful". Opposite to the ideology of a traditional giving of birth, single women using sperm donation raise connotations of consuming, "buying a baby", selecting a "father" from a catalogue of sperm donors, therefore, it is possible to consider singlemothers by choice as abandoning not only the physicality of the nuclear family ideal, but rather the ideology behind it as well (Graham, 2012).

In the past, there was a stigma related to be an unmarried mother, currently, this stigma has decreased, yet it still exists. It is possible that this change has occurred since single parent families are more common with an increase of shared residence, divorce, lesbian mothers and women who chose to become mothers. This change reflects bigger willingness of women to separate motherhood from sexual relations, parenthood and marriage (Kapell, 2014).

Studies revealed that the decision to become single-mothers is made when these women encounter a limited chance of finding a partner to bring a child with in the time frame when age 
increases, and fertility decreases, and they feel that time runs out for them to give birth to a child. Many a single mother by choice would prefer to become a mother in a context of relationship. However, should they desire to become mothers, they would not have an actual choice. The idea of being a mother through sperm donation and only later to find a partner, enables those women to get as close as possible to the picture of the ideal of nuclear family they have imagined for themselves. Despite the sadness that they cannot achieve the family they imagined for themselves, being single and experience an "urge" for motherhood means that these women need to overcome their grief and consider the aspiration of motherhood without a spouse (Graham \& Braverman, 2012).

Many are certain that the problem with single women is that they are "fastidious" and that is the reason why they do not get married (the problem is not to get married, but rather to get married with the right man), but the problem is more complex: in current age, people spend most of their time at work and the rest of social life is managed at home in front of the computer. In older age, there are not any more frames such as High school / army and university and therefore, it is very difficult to get acquainted with new people. Due to the fact that the biological clock it is "ticking" and there is no need to compromise for an unsuitable spouse, they reach a conclusion that although they want a spouse, they do not need one. This decision takes them out of the strains of pressure and despair (Matis, 2011).

The dominant culture tends to label a single-mother's action as a disaster (an accident of death or a divorce), as irresponsibility (an accident of absence of contraceptive methods), as immorality (selfish desire to improve her life) and/or not normative or anti-male (lesbian choice), in all those levels, an independent female action capability not submitted to the society's dictations is the central component in a choice of a single-mother (Kapell, 2014).

In chasing motherhood, these women do not reject the nuclear family externally, but rather reshape the ideas regarding motherhood and relations with a purpose to save at least part of the ideal of a nuclear family they have imagined for themselves. Most of the concern around women who actively aspire to single motherhood relates to norms and ideals regarding how a family should be. It is possible to consider the role of fertility treatments in creation of single-parent families as undermining the nuclear family's ideal, by reason of single-mothers no longer being circumstance (such as a collapse of nuclear family), but rather being actively created from start (Graham, 2012). 
The condition of single mothers by choice is more problematic in many ways than of other women. Indeed, those mothers are expected to achieve poverty (their chance of poverty is $30 \%$ compared with $17 \%$ in couples with children), to be unemployed, to take part-time job in order to combine professional and family life, often, they will try to work more due to lack of resources, however, it will cause them to feel very guilty as it contradicts the dominant vision of motherhood. They will have to withdraw their leisure, hobbies and passions in order to spend time with their children. If they have the means to afford for themselves an addition of childcare they will often not relay on it too much since they are afraid to neglect their children. Those mothers can have lower physical and mental health - depression rate is extremely high amongst single-mothers and they have difficulties in structuring new relationships (Heine, 2016).

Single motherhood by choice not only casts doubt on it leaving the traditional family structure but as well on undermining the perceived importance of the knowledge regarding the genetic origin of man. By aspiration for motherhood through a sperm donor, instead of having a child in a short-term relationship, they feel that not only that they will both avoid pain and confronting separation for themselves and their child but as facilitate creating a future relationship without presence of a "messy ex" (Graham, 2012).

Children to single-mothers by choice are not exposed to parental confrontations and they tend less to experience the financial distress or mental problems usually caused by collapse of marriage or as a result of an unplanned single-motherhood. However, they grow up without a father from start and those who were born from a sperm donation in fertility treatments do not know what the identity of their biological father is. Even though those mothers chose to enter single parenting, the practical difficulties of parenthood without a partner have remained. When single women aspire at motherhood by mean of an unknown donor, not only that there is an absence of physical and legal father, but there as well is no intimate knowledge about him (Golombok et al., 2016).

It was found that children who were raised in single-parent families of all types, reported on high familial consolidation and close and prolonged connection with the mother. This consolidation has double meaning, it provides something to lean on and emotional support, however as well a possible factor for difficulty in development and emotional separation (Godkar, 2009). 


\subsection{Nontraditional families in Israeli mirror}

Even though Israel is an industrialized country, traditional terms of family structure will continue to dominate Israeli society. While in Western countries, the diversity in family structures increases considerably, in Israel, the centrality of traditional nuclear family is dominant and continues being the hallmark of Israeli society. In this context, parenthood is evaluated positively, and heterosexual marriage is considered as the most legitimate frame of having children (according to data of Central Bureau of Statistics, about 95\% of couples in Israel are married). Firstly, in Israel, religious law governs matters of marriage and divorce and all these marriage arrangements are centered under religious institutions, secondly, a family is considered to be the common denominator which holds together diverse groups constituting the Israeli society. However, untraditional family models began appearing lesbians and single mothers by choice, these belong to minority groups that were forced out to the fringes of Israeli society. Having children outside traditional family model is often accepted with mixed emotions of family members and friends and it gains an insufficient support (Shechner, 2013).

The law in Israel does not recognize same-sex couples' marriages, therefore, the pattern of their lives is parallel to those who are "common-law spouses", meaning, shared life and residence without marriage. Following the recognition Israeli law awarded the "common-law spouses" institution, same-sex couples are entitled to all financial rights given to heterosexual couples. There are same-sex couples who choose to anchor their financial matters between them in a legal agreement including division of property (in case of separation) and a will (Rodin et al., 2008).

The stigmatic relation to same-sex people causes same-sex individuals and couples to hide their sexual identity and their intimacy relations and force them to lead double lives. Same-sex people in Israel still do not enjoy full equality of legal rights and they fight for their place in society. Same-sex couples are still being discriminated in marriage and in adoption of children and in July 2017, there was a media noise regarding the refusal of the State to allow same-sex couples to adopt children, in a claim that the existing law stating that "there cannot be adoption but of a man and his wife together" will not be changed in order to enable same-sex couples to adopt children and the justifications for that were: "a child sent for adoption bears a sense of abnormality and it is desired to avoid loading additional load upon the child, in an image of placing him in a family unit still perceived in the Israeli society as a new and different phenomenon". 
The Israeli society is still diffident regarding the lifestyle of the homo-lesbian community, many in the secular sector resisted the existence of the annual "pride parade" in Jerusalem with the claim that the parade and its messages undermine the religious, national and historic character of the Jewish people's capital (Rodin et al., 2008).

In Israel, where motherhood and parenthood hold a high position socially and indicate a higher status than that that of a lonesome male or female singles, single-parent families increase continuously. According to Central Bureau of Statistics, in 2015, about 20\% of all singlemothers with children are unmarried, however alongside these facts it is possible to see from an article published in April 2017 in the Israeli medical news channel (E-med) that number of "religious" hospitals, amongst them Shearei Tzedek, Maayanei Hayeshua and Leniado refuse to provide fertility treatments to women who are not married according to Jewish law, in Rabbinic courts or who are not married at all (including lesbians) despite the fact that this refusal is opposed to the law and constitutes an inappropriate usage of the medical system for sake of religious coercion.

In a single-parent family, earning capability are limited to a single provider, which puts it usually at a lower income level than the income level existing in a family where both parents work, furthermore, the fact that a woman heads most of these families, puts the family's livelihood in even greater inferiority (a gap in wages of women that is usually lower than wages of men) and therefore, due to the great difficulty imposed on single mothers, the state of Israel legislated in 1992 a Single-Family Law (in 2014 the name of the law was changed to "Assistance to Families Headed by an Independent Parent Law") which defines who is a singleparent and what are the benefits that will be given to them by The National Insurance Institute as part of this law, following are the rights as given as part of the law:

- Guaranteed income guarantee pension - which guarantees single-mothers a possibility to provide for their children in dignity and provide for them the most basic things they need.

- Help with housing - eligibility for aid in financing of rent, discount of $20 \%$ in city taxes.

- Employment - single-mothers are entitled to receive credit points in income tax (should they work) and are entitled to be absent more from their work due to illness of a child.

- Education - single mothers to children of 6-14 years of age are entitled to a grant for studies, as well as discount in tuition fees, for children's after-curricular activities and help in financing summer day camps. 
In summary, the state of Israel is at a crossroad between keeping norms of traditional nuclear family structure and the appearance of alternative family models created as a result of the influence of post-modern social processes (Shechner, 2013).

\subsection{Policy of fertility and birth in Israel}

Israel is a unique country which is defined legally as being Jewish and democratic with strong emphasis on the political and legal power of religious law tradition especially in matters of family laws and is identified with pro-birth ideology, meaning, an array of beliefs, views and practices encouraging birth, elevating the role of parenthood and emphasize the benefits of giving birth to children and raising thereof. Under the cover of this ideology, a comprehensive cultural work is being done in Israel which is enlisted for birth encouragement, and by virtue of this fact, parenthood is perceived as extensive cultural default. Worldwide demographic data teach that in recent decades, the expectation for birth of children erodes: in the Western world the rate of those who want a child decreases more than at any other time in history and the Western population faces negative birth rates and depopulation (Donath, 2015).

According to Central Bureau of Statistics, comprehensive fertility rate in Israel is the highest amongst developing countries and in 2011 it was 3 as compared to 1.7 in OECD countries and birthrate in Israel for 2013 was $28 \%$ and is the second amongst OECD countries.

The attitude of the State and its policy to encourage birth stems mainly from its strong tradition molded by the Biblical obligation and collective duty "be fruitful and multiply" accompanied by stories of Biblical mothers: Sara, Rachel, Rebecca and the desperate desire of Hanna for children. This commandment is important and central, and it considers parenthood a moral and religious decree. Infertility of a woman is an architype of suffering in Jewish imagination and a man who has no children is as good as dead. Be fruitful and multiply commandment meets the demographic balance threat and together they are organized around the horrible memory of the holocaust, the consistent loss of life in terrorist attacks and military battles, and the fear of demographic threat caused by Arab countries surrounding the country (Sperling, 2010).

The religious national demographic narrative reveals that children are perceived as a national asset due to their essentiality for continuation of society and realization of biological motherhood in Israel is perceived first and foremost as basic civilian moral duty and as a patriotic mission of highest degree. Coercion of motherhood is operating in a way that lack thereof might be considered almost as a national treason. An additional aspect sees a tight linkage between children and happiness and since a woman can be mother, it was concluded 
that not only does she need to be a mother, but she as well must be, and that only in motherhood can she find her happiness. In Israel, the condition of happiness and birth is conclusive and unequivocal even more and there is a belief that people who do not have children have an empty life. Rearing of children is such a definite norm in the State that the variety of experiences connected with it are perceived as an inseparable part of the course of life (Donath, 2015).

Despite the fact that according to Jewish law a man or a woman who cannot be fruitful and multiply due to medical problems are exempted from this commandment, and due to the strong Jewish ethos of a mental will to have children, couples and even individuals make great efforts - health, medical and financial in order to have the privilege and bring children to the world (Viner-Uman, 2012).

The rate of pregnancy terminations in Israel is low as compared with most West European countries. According to law, a woman has no general right to terminate her pregnancy and pregnancy termination can occur under specific circumstances by pregnancy termination committees operating in medical institutions by virtue of "Penal Law" from 1977, which were established by hospitals or the Ministry of Health. The Law permits pregnancy termination in following cases (From pregnancy terminations according to law 1990-2013, Ministry of Health):

- The woman is below 17 or over 40 years of age.

- The pregnancy stems from relationship forbidden by law, from incest or outside of wedlock.

- The embryo might have physical or mental defect.

- Continuation of pregnancy might jeopardize the woman's life or cause her physical or mental damage.

An article published this year, in the medical journal (E-med), reveals that those hospitals defined as religious, refuse to in practice perform pregnancy termination out of religious reasons, and do not approve the procedure in the pregnancy termination committees. In Laniado hospital in Netanya, for example, there is no pregnancy termination committee at all, as the hospital opposes performing abortions out of religious reasons and in the other two hospitals (Shearei Tzedek and Maayanei Hayeshua) there is a pregnancy termination committee, however, women who are interested are turned to a committee in another hospital as the committee in these hospitals approves abortions only in cases of severe defects in an embryo, 
or in cases of life endangerment and do not approve abortions out of other reasons which are legally approved and this, naturally, is due to a religious cause (as according to Jewish law, an embryo is considered as a living creature from the moment of its creation and it is forbidden to hurt it in any way).

In the Israeli society, the subject of fertility and birth has enormous social and cultural significance. Parenthood is perceived as one of the indexes of success and is considered as the basic calling of humans. A deliberate and utter renunciation of parenthood is still an uncommon phenomenon in Israel. Usually, the society recognizes the right of a person to get married and have children in a sense that his way of structuring a family is not to be blocked (freedom of procreation), however, in Israel, there is a claim towards the State not only to enable having a family but actually to assist, meaning, supplying medical service in realization of the right of parenthood. This issue is significantly expressed in acknowledging the duty of the State to finance fertility treatments in a scope that does not exist in other countries. The great number of treatment cycles as well expresses in case of old single mothers conceiving both from egg donation and sperm donation and undergoing more than 17 treatment cycles per woman. It is not surprising that in Israel there are more fertility clinics per capita than in any other country. The excessive data of IVF treatments and use of fertility treatments in Israel are supported by the pro-birth approach of the State to advance the liberty of procreation (Heyd, 2011).

The Ministry of Health regulation enables use of IVF and PGD (Preimplantation Genetic diagnosis) to choose the sex of the future newborn for medical and nonmedical purposes, there are precedents enabling to produce a sperm from a dead person (including parents' request for a soldier who was killed in a terrorist attack) and use of sperm deposited prior to the person's death. Professional instructions enable, under certain conditions, a transfer of up to four fertilized eggs to the womb, they as well authorize execution of insemination for a married woman who undergoes divorce proceedings. The Supreme Court issued a warrant in favor of a request of a political murderer to perform in-vitro fertilization for his wife under security limitations, however, rejected surrogacy of an unmarried women. Even though according to the law, it being a discrimination and violation of the principle of equality (Sperling, 2010).

A pro-birth attitude in Israel is best expressed in law and policy that provide: birth services and free hospitals, birth grants for new mothers and significant child pensions for large families which are an additional means to promote the public interest to encourage procreation and birth. It is not surprising that in such social, political and cultural environment, women who have no 
children in Israel suffer from a prolonged stigma that can only be remedied using fertility treatments / IVF (Sperling, 2010).

\section{Infertility}

Infertility is defined as failure to conceive after 12 months of orderly intercourse without use of any contraceptive methods in women under age 35 and after 6 months of orderly intercourse without use of any contraceptive methods in women of 35 years of age and above and regarding women over 40 years of age, even less than 6 months of attempts to conceive without success (Kuohung \& Hornstein, 2016).

This is a phenomenon of the new millennium and the estimation is that its incidence is at 9$12 \%$ amongst couple in fertility age around the world, nonetheless, in some areas of the world rates of infertility are much higher and reach even $30 \%$ in some populations, including: SouthEastern Asia, Africa south to the Sahara, Middle East and North America, Central and Eastern Europe and Central Asia (Inhorn \& Patrizio, 2015).

According to data of ASRM (American Society for Reproductive Medicine, 2012) in 2010, $1.9 \%$ of women in the world of age $20-44$, encountered infertility problems and $10.5 \%$ of women who were pregnant in the past could not conceive again.

Fertility of men and women is age dependent. In women, the extent of their fertility begins to drop as early as in their early 30's and plummets sharply at the end of their 30's following a decrease in number of eggs remaining in the ovary that is normal for the age. As opposed to decrease of fertility in a woman as her age increases, in a man, reduction in sperm characteristics occurs in a much later age. Although the quality of sperm reduces slightly as a man ages, however, this usually does not constitute a problem for a man prior to the age of 60 . The change is also not felt sharply in men as it is in women, changes in fertility and sexual functioning occur in men as they get older - their testicles become softer and morphology of semen and its motility tend to drop. Despite these changes, there is no maximal age in which a man can fertilize as testified by men in their 60's and 70's who became fathers from their young spouses.

In a book by Hashiloni-Dolev (2013), in current society, age-dependent fertility becomes more common due to a wide variety of reasons such as postponing the age of marriage, many women wait until the age of 30 in order to begin family life. In studies conducted in Israel, Europe and USA, which examined the reasons for postponing the age of parenting, it was found that the more women are educated they tend more to postpone their first delivery to an older age. For 
them, a late entry to parenthood constitutes a way to reduce the conflict between career and motherhood which is the central conflict in a life of a modern woman.

The diagnosis of infertility in the literature refers to heterosexual couples, because most medical definitions of infertility rely upon heterosexual activity, whereas single women and lesbians, infertility is not necessarily a reason referring to treatment, but the social context which does not allow getting pregnant naturally - called in the literature- Social Infertility. This term is used to refer to individuals who are unable to reproduce due to relationship status, so in a sense, lesbian and single women are all infertile (Lo \& Campo-Engelstein, 2018).

\subsection{Common factors of infertility according to (Kuohung \& Hornstein, 2016).}

- Interruption in ovulation - $25 \%$ - seldom ovulation, or absence of ovulation causes infertility, as the egg is not available every month for fertilization. Absence of orderly ovulation can bring about difficulty in pregnancy.

- Aging in eggs - dropping in fertility capability with age, apparently due to reduction in number of eggs and their quality.

- Endometriosis - $15 \%$ - anatomic deformity that causes pelvic adhesions and damage to ovarian tissue. Production of materials (such as cytokines) that harm the regular process of ovulation, fertilization, and implantation of the embryo.

- Pelvic adhesions - $12 \%$ - due to previous surgeries (abdomen opening and caesarian section), endometriosis, pelvic inflammation - PID (because of intrauterine device or performing, chronic bowel inflammation.

- Obstruction of fallopian tubes - $11 \%$ - obstruction in a fallopian tube prevents a normal passage of ta egg and the semen through the fallopian tube. The primary factor of obstruction of fallopian tubes is pelvic inflammatory disease - PID, additional factors can be endometriosis, post-surgery adhesion or inflammation of fallopian tubes.

- Other deficiencies in fallopian tubes - $11 \%$ - hydrosalpinx - reduces fertility by returning of fallopian content tube liquid into the uterine cavity and thus creating a hostile environment for embryo implantation.

- Prolactin surplus - 7\% - prolactin is milk hormone. Its increased secretion often causes infertility and ovulatory disorder. 
- Cervix factors - 3\% - in ovulation, cervical mucus facilitates sperm motility. Congenital defects and cervical trauma might cause narrowing and inability of the cervix to create normal mucus and thus harm fertility.

Additional factors:

- PCOs - polycystic ovary syndrome - is a common hormonal disorder that affects 5$10 \%$ of women. This syndrome is a collection of problems that are revealed mutually. Not all women with PCOs have all the symptoms. In order to diagnose PCOs, a woman must have 2 out of 3 possible problems: chronic deficiency of absence of ovulation, high chronic levels of testosterone hormone and ovaries that have a large number of small follicles (American Society of Reproductive Medicine, 2014).

- Womb anomalies - considered to be factors of infertility by disorder of the normal implantation. Other defects in uterus structure that are related to infertility include polyps in womb mucus and adhesion as a result of curettage).

- Vaccination factors - for example Celiac disease. Women with an untreated Celiac disease might have a higher incidence of reproductive defects such as infertility and abortions.

- Genetic factors - childless couples have a higher incidence of karyotype defects. The commonest of which that is related to infertility is Terner syndrome (45X0) According to data of American Society for Reproductive Medicine (ASRM), in her 30's, the chances of a woman to conceive each month is at $20 \%$ whereas in her 40 's the chance is less than 5\% each month. Kuohung and Hornstein (2016) defined infertility as a common condition which has psychiatric, economic demographic and medical important implications and its factors can be attributed to a woman (37\%) a man (8\%), combined problems of woman \& man (35\%) or an inexplicable factor $(28 \%)$. Another factor of infertility that comprises $8 \%$ of all the problems, is a problem in a couple's intercourse.

\subsection{Infertility factors of male origin according to (Swerdolff \& Wang, 2016).}

- Unknown cause - 30-40\% - despite a cautious estimation of various possible mechanical reasons, a factor of improper sperm count, morphology or motility cannot be identified in a part of infertile men. While many a man with infertility has oligospermia (low number if sperm cells in relation to range) or azoospermia (no sperm cells at all), part of infertile men have normal sperm count. Over $80 \%$ of men with 
infertility problems have low sperm concentration related to drop in sperm motility and proper morphology, others might have reduction of sperm motility and improper morphology.

- Sperm transport disorders - 10-12\% - causes reduction of male fertility due to conditions that affect male genital system after production of sperm in the testicles and includes defects in genital tract as well as ejaculation problems. For example: cryptorchidism - a man with a history of cryptorchidism has low sperm count, low sperm quality and low fertility rates.

- Varicocele - expansion of testicle veins in the scrotum. Varicocele on the left side is 10 times more common than on the right side, might be due to an anatomic structure.

- Vas deferens defects - male infertility can be caused by congenital or acquired defects in the vas deferens. Bilateral obstruction of the vas deferens can be caused by inflammation (chlamydia infection / tuberculosis).

- Retrograde ejaculation - a condition in which sperm enters the bladder instead of getting outside, there is practically no ejaculation and therefore no fertility capability. This phenomenon can be caused by use of medical drugs, surgeries in the area that damage innervation or damage to muscles.

- Genetic disorder of sperm production - 10-20\% - although infertility of male origin is a common disorder, a number of genetic factors were identified only recently by special techniques. Severe disorders in sperm morphology were identified as genetic mutations in men with severe disorders to sperm morphology.

- $\mathrm{Y}$ chromosome and disorders relating to linkage - defect in $\mathrm{Y}$ chromosome is more identified with a genetic factor for small quantity of sperm cells and severe absence of sperm motility.

- Endocrine and systemic disorders - 10-20\% - testicle disease - primary defects in testicles in sperm production - is the most significant factor of absence of sperm cells or a small quantity of sperm cells. The factor of lack of testicle functionality is unknown and can fit a congenital defect in sperm production or as a response to an event in the past.

Additional factors:

- Genetic factors - Klinefelter syndrome (XXY) one of the most common reasons for hypogonadism that can occur in 10-15\% of infertile men with absence of sperm cells and small testicles. 
- Acquired testicle disorder - almost any disorder acquired in the testicles can cause infertility, for example - viral infection particularly mumps/tuberculosis.

- Medical drugs and radiation - many a medical drug is related to delay in sperm production. Ionized radiation delays sperm production - low levels temporarily suppress sperm production whereas high levels of radiation cause absence of sperm cells and irreversible infertility.

\subsection{Unexplained infertility}

When couples are not able to get pregnant with no identifiable reason, about $30 \%$ of infertility are defined as unexplained infertility and is a multifocal phenomenon of reproduction. Despite significant improvement made in diagnosis and care of reproduction, many couples still have no explanation for its infertility even after a series of most accurate diagnostical tests (Galliano \& Pellicer, 2015). There is no uniform definition of unexplained infertility and it changes in literature and administration of therapy depends upon length of infertility period and age of the female spouse (Ray, Shah, Gudi \& Homburg, 2012). Many an unexplained infertility is apparently caused by presence of multiple factors (for example - female spouse of over 35 years of age with low ovary reserve and male spouse with low parameters of normal sperm) that each one in itself does not significantly reduce fertility, however can reduce the number of pregnancies (Kuohung \& Hornstein, 2016).

\subsection{Isolation and evaluation of infertility}

Isolation is usually performed after one year of unprotected regular intercourse in women under the age of 35 and after 6 months of unprotected intercourse in women of 35 years of age and above. Nonetheless, the evaluation might be in an earlier time in women with irregular menstrual cycles, or with known risk factors of infertility, such as: endometriosis, history of PID (Pelvic Inflammatory Disease), or defect in the reproductive system. Evaluation of basic infertility can be performed by a family doctor or an experienced gynecologist. A family doctor usually refers the patient to a specialist doctor for fertility treatments whereas many a gynecologist tends occasionally to begin with treatments even prior to referral to a fertility specialist doctor. This decision depends upon the results of tests and experience of provider of the treatment. It is important to remember that the couple might have many factors that cause their infertility and therefore, the full initial diagnostical evaluation should be done to identify the most common factors of fertility problems should they exist. Evaluation of both partners is to be performed in parallel (Kuohung \& Hornstein, 2016). 


\subsubsection{Evaluation of infertility in a woman}

History and physical examination - findings on history and physical examination can indicate the cause of infertility and thus assist focusing the diagnosis. Physical examination is conducted to isolate signs for potential causes of infertility. The BMI (Body Mass Index) of a patient is to be considered - extreme BMI is related to low fertility and improper weight gain is related to insulin resistance.

Primary points in history:

- Length of infertility and results of prior evaluations and treatments.

- Menstrual history (length of menses and its character) - that assists in determining the ovulation status.

- Medical, surgery, genecology history (including diseases transmitted by sexual contact - STD, pelvic inflammatory disease and treatment following abnormal PAP tests such as conization. Searching for procedures or medications that might be related to infertility.

- Midwifery history for events with potential of infertility.

- Sexual history - including problems in sexual performance and frequency of sexual intercourse. Too seldom or ineffective intercourse can serve as an explanation for infertility.

- Personal history and lifestyle - including age, profession, physical exercise, tension, weight changes - diet, smoking and drinking alcohol that might harm fertility (Swerdolff \& Wang, 2016).

Evaluation of ovulation functioning - this evaluation is a central component of evaluation of the female spouse, due to the fact that ovulation problems are a common factor of fertility problems. Women with regular menstruation periods of 28 days with symptoms such as: breast sensitivity, swelling, tiredness, etc., are likely to assume that they ovulate. In women who do not describe their period as such, an evaluation is to be conducted by blood tests in a lab. Ovulation is easily documented by level of progesterone in the luteal-middle phase occurring about a week before arrival of the expected menstrual cycle. For a typical cycle of 28 days, the test will be performed on the $21^{\text {st }}$ day. Progesterone with a level of higher than $8 \mathrm{pmol} / \mathrm{ml}$ is an evidence of ovulation recently occurred. Another alternative is to propose a patient to use a kit for prediction of ovulation (urine test). This kit is not effective for identifying the LH hormone, an increase of which predicts ovulation. A more exact method of determining ovulation is 
performed by daily ultrasound in order to monitor development of the follicle and its eventual disappearance (Kuohung \& Hornstein, 2016).

Evaluation of ovarian reserve - a low ovarian reserve can relate to a reduced quality of eggs, amount of eggs or reproduction potential. Identification of ovarian reserve is a most important component of primary evaluation. In women over age of 35, or women with risk factors of early ovary failure, the ovarian reserve is tested according to Follicle Stimulation Hormone (FSH) and estradiol in blood on $3^{\text {rd }}$ day of the menstrual cycle (whereas the first day is the first day of menses). The basic assumption of this test is that women with ovarian reserve have sufficient production of hormones in the ovaries from small follicles in the early phase of the menstrual cycle to maintain a low level of FSH. Oppositely, women with a reduced reserve of follicles and eggs do not have enough production of hormones in the ovaries in order to provide a normal delay of FSH secretion from the pituitary gland that brings about a rise in FSH levels.

A proper FSH concentration in $3^{\text {rd }}$ day that should be below 10 IU/I, implies a sufficient ovarian reserve, whereas a level of 10-15 IU/I is borderline. The level of estradiol is tested as well on the $3^{\text {rd }}$ day (a value of $<80$ implies a proper ovarian reserve), although there are contradictory data regarding the question whether this is an index of ovarian reserve prediction. High levels of estradiol are caused by early enlisting of follicles that occurs in women with very low ovarian reserve. High estradiol levels might delay production of FSH from the pituitary gland and thus mask one of the signs of reduction in ovarian reserve in women around the menopause age (Kuohung \& Hornstein, 2016).

Anti-Mullerian Hormone (AMH) - a hormone produced by immature follicles in the ovary that contain eggs. Amount of AMH gives an indication of the number of eggs to be developed, or an ovarian reserve and it can be tested by a simple blood test in any phase of the female menstrual cycle. Amount of AMH measurable in the blood remains permanent until early maturity and from age 25 its level begins to drop. From age 35, the level of AMH continually reduces until it reaches 0 in menopause age. Interpretations of levels of AMH are lab-dependent and there is no international standard (City Fertility Center website).

Hysteroscopy - is a test performed in the first half of the menstrual cycle (between end of menses and before ovulation time) in which a telescope is inserted into the uterine cavity through the vagina and the cervix in order to demonstrate the uterine cavity as well as the Fallopian tubes openings, the cervix canal and the vagina. Washing liquid is dripped - saline (which is most suitable as a medium for inflating the cavity) in order to well inflate the cavity 
to allow for a proper endoscopic view and thus it is possible to give a pathological evaluation of the uterine cavity (Mencaglia \& Calvacanti \& Alvarez, 2013). Should abnormal findings be revealed in diagnostic hysteroscopy, or in ultrasound test, a surgical hysteroscopy is performed which is an invasive action performed under general anesthesia, and a one day hospitalization. In the process of surgical hysteroscopy, an electrode is attached through which tissue, myomas, polyps and remains of pregnancy can be cut, as well as adhesions or congenital defects such as a septum (Goldshmit, H. Hysteroscopy and Laparoscopy website).

It is not possible to evaluate by means of pathological hysteroscopy in the Fallopian tubes, or external outlines of the uterus and the middle layer of uterine wall and thus it is insufficient in order to evaluate these anatomic structures as part of an infertility evaluation. For this purpose, hysterosalpingography is performed (Bradley, 2016).

Hysterosalpingography (HSG) - is intended for ruling out obstruction of Fallopian tubes and for evaluating the uterus and cavity structure. A contrast material (iodine) is employed in order to demonstrate the uterus structure and Fallopian tubes openings. After injection of the contrast material, a series of X-ray pictures is conducted in order to follow up on dispersal of the contrast material which clearly demonstrates the uterine cavity and if the Fallopian tubes are open, the contrast material will disperse into the Uterine cavity, and should they not, the contrast material will accumulate in the Fallopian tube. Hysterosalpingography has a therapy aspect as well - in most cases a obstruction in the Fallopian tube can be opened by air pressure.

Hysterosalpingography is not effective for locating adhesions in the Fallopian tubes or endometriosis. Defects found in hysterosalpingography usually require further clarification by additional imaging means such as a 3D ultrasound (Kuohung \& Hornstein, 2016).

Karyotyping - performed on women with exceedingly early menopause (before the age of 40) and on both partners, should they have a history of repeating miscarriages in their past (Kuohung \& Hornstein, 2016).

\subsubsection{Evaluating infertility in a man}

According to the article of Swerdloff and Wang (2016) evaluation of infertility of a male spouse is frustrating both to a patient and a therapist as only in a small number of men, etiology or specific treatment can be located. Disorders in most men are characterized by description of abnormalities such as: reduction in number of sperm cells, motility, or ability to fertilize an egg. Evaluation of a sterile man should begin with a detailed history focusing on the potential cause of infertility. 
Components of the evaluation include:

- Developmental history - including testicle descent, puberty, hair loss or excessive hair, or reduction in shaving frequency.

- Chronic medical deceases.

- Inflammations, such as: mumps, STD and urinary and genital tract infections including prostate.

- Surgical procedures involving the groin and scrotum areas.

- Sexual history - including libido, frequency of sexual intercourse and evaluation of previous fertility of the man and his spouse (Swerdolff \& Wang, 2016).

General physical examination is intended to locate evidence of androgen deficiency that might be attending reduction of fertility. Examination of a man should include the following components:

- General appearance - torso/lower body ratio indicates androgen deficiency that preceded maturation. On the other hand, a rise in body mass (fat) and decrease in muscle mass testifies to current androgen deficiency.

- Skin - loss of pubic, axillary, and facial hair testifies to overtime androgen deficiency.

- External genitals - disorders that might affect fertility can be located by examination of external genitals. Diseases that affect sperm maturity and transport thereof can be identified by examination of the scrotum such as varicocele.

- Breasts - breast enlargement in a man (gynecomastia) can imply reduction in androgen in relation to estrogen.

Semen analysis - is the cornerstone of male evaluation as part of infertility examination. Standard semen analysis is composed of the following indexes:

- Measuring volume of seminal fluid and PH.

- Microscopy for searching for waste and viscosity

- Evaluation of semen concentration, motility, and morphology.

- Leukocytes count in semen (Swerdolff \& Wang, 2016).

It is common knowledge that in recent decades, sperm quality in normal men has dropped. Furthermore, meta-analysis of reports published around the world, revealed that over past decades, there has been a trend of decrease in semen count as well as in volume of seminal fluid, despite known factors, such as: specific deceases, chronic injuries, health problems and lifestyle that can lead to male infertility, unknown factors (infertility due to idiopathic male 
factor) play a key role in this global problem. Studies conducted recently reveal that male infertility can stem from factors such as reduced semen production or deformed semen, or lack of motility of sperm cells can be related to human exposure to electromagnetic fields (EMFs). Many a study revealed that exposure to common sources of EMF such as: cellphones, phone chargers, laptops that are connected to wireless internet with low frequency or exposure to electromagnetic frequency of very low frequency have caused a reduction in quality of human sperm (Mahmoudi et al., 2015).

Semen analysis should be performed after 2-7 days of abstaining from sexual intercourse, it can be delivered directly into a sterile cup (by musterbation) and when it is not possible, the sample can be collected in a condom with no chemical additives and be delivered to a lab within an hour from delivery of the sample. Due to the nature of semen analysis, minimally two tests should be performed within a gap of one to two weeks between them.

Semen analysis is performed by standardization methods such as those of WHO (World Health Organization).

Reference values for the analysis:

- Volume - $1.5 \mathrm{ml}$

- Concentration $-15 * 10$ million sperm cells

- Total number of sperm cells - 39 million

- Morphology $-4 \%$ undamaged cells

- Vitality $-58 \%$ live

- Progressive motility - 32\%

- Total - progressive and local motility - 40\% (Swerdolff \& Wang, 2016).

Low volume and concentration with presence of azoospermia (with no presence of sperm cells) or severe oligospermia (minority of sperm cells) indicates an obstruction in genital tract. Low sperm volume with a proper sperm concentration indicates, apparently, a problem of sperm collection and partial retrograde ejaculation. Androgen deficiency is as well related to low sperm volume and low sperm concentration. Some men with sperm count that is considered to be low, can be fertile while others above bottom limit or normal can be infertile. Normal morphology criteria based in the past mainly on form as observed microscopically. Now they include the area as well that sperm cells take up by acrosome and neck and tail defects. Sperm motility is evaluated by observing through a microscope and is classified as: progressive motility, local motility and no motility. At least $40 \%$ of sperm cells need to have motility and at least $32 \%$ of them to have progressive motility. 
Leukocytes - while blood cells, are often present in sperm cells, however a large presence of white blood cells in the fluid might be a sign of genital inflammation and might be related to low sperm quality. Sperm culture is often performed in men whose semen analysis contains inflammatory cells; however, the results are usually unanalyzable. Over viscosity can interfere with semen analysis and evaluation of sperm motility particularly, despite the fact that the cause of over viscosity is unclear, it is evaluated that it is caused by inflammation of genital and urinary tracts (Swerdolff \& Wang, 2016).

Post coital Test (PCT) - is a test performed in middle of menstrual cycle, usually around 10$12^{\text {th }}$ day (should the cycle be a 28-day cycle) the purpose of the test is primarily evaluating the cervical mucus of a woman and secondarily, estimating sperm functioning of the spouse in the mucus (number of sperm cells and their motility). Should the test be performed in the morning, intercourse should be performed in previous night. Appearance of motile sperm cells (under a microscope) in the cervical mucus is considered a normal PCT, lack of presence of sperm cells and immotile sperm cells, is considered as not proper. PCT test as part of infertility evaluation, can give an idea whether a cervical factor is the cause of infertility (FertilityBristol.com, 2014). Endocrine test - evaluation of an infertile man includes a test of values of the following hormones: testosterone, LH and FSH. When concentration of testosterone in a serum is low, concentrations of FSH and LH in the serum rise which indicates a primary hypogonadism (caused by lack of testosterone production related to damage to sexual functioning) and when the values are low or normal, this indicates secondary hypogonadism (Swerdolff \& Wang, 2016).

\subsection{Criteria for fertility treatments}

After isolation of the cause of infertility, a treatment is given, which purpose is to correct factors of reversible source and overcome irreversible factors. In the beginning of the meeting, the couple is consulted to perform changes in lifestyle in order to improve fertility, such as: stopping smoking, reducing excessive consumption of caffeine and alcohol and frequency of sexual intercourse (every day or two around the expected ovulation time (Kouhung \& Hornstein, 2016).

Israel, as a pro-natalist country is the only one in the world that funds fertility treatments for its citizens in full - whether they are married couples, single women, lesbian women, Jews or Arabs. Fertility treatments are funded up to the late age of 45 for a woman attempted to conceive through her own eggs and even to a later age for women assisted by egg donation. The funding 
is extended with no bearing to financial situation, emotional condition, level of education of those desiring to become parents. The funding is all-inclusive, to the extent that the State finances treatments as well to couples who have children from previous marriages up to the birth of two mutual children. Nonetheless, despite the subsidy, a deductible is still required (Hashiloni-Dolev, 2013).

In term of absence from work for fertility treatments, employees who undergo fertility treatments are eligible to be absent from work on account of sick leave accumulated for them (and that according to advance notice on the absences). The maximal number of days in which an employee is eligible to be absent due to treatments is: for those who work 5 days a week, up to 64 days annually, on account of sick leave and for those working 6 days a week, up to 80 days annually, on account of sick leave. Additionally, in a period of absence due to treatments and for the period of 150 days afterwards, an employer cannot fire those employees or hurt their wages (any privilege).

Patients are to be involved in possibilities of fertility treatments. These possibilities involve 4 primary factors: effectiveness (rate of live birth), liability of treatment (frequency of injections and visits in a clinic), safety (risk of ovarian over-stimulation and multi-fetal pregnancy) and financial expenses for treatments. Categoric contraindications of fertility treatments are contraindications of pregnancy and against the use of medication in order to improve fertility. Marital status of parents, sexual preference, HIV decease, should not constitute a factor according which fertility treatments are being prevented (Kouhong \& Hornstein, 2016).

Since the cause of infertility is more common amongst women, it is customary to examine thoroughly the female spouse and treat her while the male spouse undergoes clarification. Problems in a female spouse, such as lack of ovulation, irregular ovulation, high levels of prolactin, endometriosis and obstruction of Fallopian tubes necessitates medication treatments concurrently or even prior to treating the male spouse. Until recently, managing infertility of a male factor was a frustrating experience both to a therapist and a patient due to a reduced understanding of pathogenesis of infertility and lack of ability to treat in most cases of male infertility. Thus, treating the female spouse can often compensate infertility of a male factor, such as low to medium drop in sperm parameters and as a result, achieve pregnancy without treating the male partner (Swerdloff \& Wang, 2016). 


\subsubsection{Treating infertility of female source}

In an article by Kouhung \& Hornstein (2016), a woman with deficit of eggs that is not related to ovarian malfunction, can usually be treated with medication for ovulation induction. These women achieve fertility capability almost identically to that of a normal couple (meaning 15$25 \%$ probability of achieving pregnancy in a single menstrual monthly cycle). Nonetheless, ability to conceive is achieved on the account of an elevated risk of multi-fetal pregnancy. The chosen treatment of ovulation induction should be based on the basic cause of absence of ovulation and on effectiveness, risks and potential complications attributed to every treatment and that are adjusted to each woman. The possibilities include:

- Weight regulation - women with a high basic weight or with BMI above 27, who do not ovulate, are advised to lose weight. For fat women with polycystic ovaries, loss of $5-10 \%$ of body weight is sufficient in order to regain ovulation in $55-100 \%$ of these women within 6 months. Weight loss is cheap, low level in interference, with no side effects and with other health advantages and therefore, should be a first-line treatment.

- Women who do not ovulate, with low BMI (lower than 7), with eating disorders or who perform strenuous physical activity, might develop hypogonadism (disorder in secretion of LH and FSH hormones from the hypophysis or the hypothalamus - Israeli Journal of Pediatrics, 2009) or even amenorrhea. Psychological stress might also disrupt secretion of GnRh hormone and thus harm ovary functioning. These women are advised to gain weight, change diet, and reduce physical exercise. Nonetheless, these women usually refuse to change their conduct.

- Ovulation monitoring - young couples with less than 12 months of attempts to conceive are advised to focus the initial interference on monitoring natural ovulation by means of ovulation kit in urine or by performing ultrasound and blood tests in order to locate the time of ovulation and to direct intercourse in the right time. And are advised waiting at least 12 months if no pregnancy.

- Metformin - for women with PCOs, insulin resistance is a common phenomenon. Remedying hyperinsulinemia with metformin has a positive effect in women with PCOS who do not ovulate, as it leads to a rise in menstrual cyclicity and improves spontaneous ovulation.

- Clomiphene citrate -is anti-estrogen that affects ovulation. Clomiphene in itself does not cause ovulation directly, but rather increases the effect of negative feedback on receptors of estrogens in hypothalamus and brings about a secretion of GnRh hormone 
which brings about the secretion of gonadotrophin hormones from the hypophysis gland $(\mathrm{FSH} / \mathrm{LH})$ that operate on the ovaries for induction and growing of follicles. This treatment is given to women who do not ovulate or those with minority of ovulations. The treatment is begun on $3-5^{\text {th }}$ day of the menstrual cycle, it is common to begin with one or two tablets a day for 5 days. Should it be required, it is possible to administer up to 3 pills a day $(150 \mathrm{mg})$ which is the maximal dosage. Clomiphene has negative effect on the cervical mucus due to a rise in its viscosity, so that in $30 \%$ of the cases there is a negative effect for achieving pregnancy.

Side effects:

Heat waves $-10 \%$

Abdominal pain and swelling $-5.5 \%$

Nausea and vomiting $-2.2 \%$

Chest congestion $-2 \%$

Vision disorders $-1.5 \%$

Headaches $-1.3 \%$

Dryness and hair loss $-0.3 \%$

Percentage of multi-fetal pregnancies in such a treatment is very low. About $80 \%$ would ovulation following the taking of clomiphene and about $40 \%$ of the women would conceive following the treatment. It is customary to perform 3 cycles of treatment with clomiphene. And should ovulation or pregnancy not have been achieved; one moves to gonadotrophins treatment protocol (Caremed website).

Gonadotrophins - since their introduction to the clinical practice in 1961, gonadotrophins have been produced from urine of women in menopause and constituted a central role in ovulation induction. Refining of the product brought about availability of particularly pure and clean FSH and LH (Menogon / Menopur). Since 1996, the human recombinant FSH are being used which are easy to operate (subcutaneous rather than intramuscular injection) and are purer (Gonal-F/ Puregon) (Fauser, 2016). The medication is administered by subcutaneous injection daily and usually from $3-5^{\text {th }}$ day of the menstrual cycle. Injection of the medicine can be performed by a nurse or by self-injection of a patient or a relative. Length of time of treatment and medication dosage is determined personally for each woman according to her age, weight, her ovary structure and response to past treatment. In comparison to clomiphene treatment, injection 
treatment necessitates a severe monitoring of the ovarian response through monitoring of follicles through ultrasound and blood test for level of estradiol and progesterone. Usually, monitoring begins after 4-5 injections and continues every two or three days. Majority of women would ovulate after 7-14 days of injections. The purpose of the treatment in a woman who does not ovulate at all, is to cause ovulation of a single follicle, oppositely, in women with regular ovulations, the purpose of the treatemet is to create "super-ovulation" of a number of follicles and thus raise the chance of pregnancy (Caremed website).

In a paper of Fauser (2016), should three follicles or more develop with diameter of $15 \mathrm{~mm}$, ovary stimulation is to be ceased and administer a medicine of immediate suppression of LH antagonist in order to prevent independent ovulation and avoid ovarian over-stimulation and multi-fetal pregnancy. Measurements of estradiol levels in blood have usefulness in order to predict ovarian over-stimulation (when it is higher than the normal range).

Common side effects: lower-abdominal pain, nausea, vomiting, diarrhea, sense of swelling, sense of shortness of breath, edemas in limbs, weight gain, headaches, chills and pimples. In most cases, ovulation is achieved, similar rate of success in women who ovulate spontaneously.

Multiple fetuses - up to $35 \%$ (5\% triplets or more)

Embryo reduction - 3-10\% of pregnancy loss

Spontaneous miscarriages $-25 \%$

Ovarian over-stimulation $-1-10 \%$

Severe ovarian stimulation $-0.1-10 \%$

Multiplicity of extrauterine pregnancy (Mavor, 2015).

The chance for pregnancy is conditioned by a woman's age, sperm details and other fertility problems. However, the chance to conceive in each cycle is at $20 \%$, so that over half of the couples would achieve pregnancy after 3 treatment cycles (Caremed website).

- Human Chorionic Gonadotropin - (Obetrol) administered as causing ovulation when at least one follicle seems ripe, when its diameter is $18 \mathrm{~mm}$ and/or level of estradiol in blood is at $734 \mathrm{pml} / \mathrm{L}$. Ovulation takes place about 36 hours after administration of obetrol. Obetrol can as well be administered as a support of the corpus luteum after ovulation. Obetrol is administered by subcutaneous injection in dosage of $250 \mathrm{mg}$. Inducing ovulation is almost certain should Obetrol be administered in right timing (Caremed website). 


\subsubsection{Infertility treatment of male origin}

Regarding infertility treatment of male origin - there is a variety of reasons for infertility that is irreversible, with no available treatment, for example, there is no known treatment for encouraging sperm production when vas deferens is severely damaged. A condition, in which the testicles produce a small amount of sex hormones or no production at all, is called Hypogonadotropic hypogonadism and it can be brought about due to following reason: should the condition be derived from excessive prolactin in the blood, it is possible to correct the hypogonadotropic condition and rehabilitate fertility by lowering prolactin concentration in the blood.

Normal production of sperm cells takes three months, in this case, renewal of sperm count does not take place for 3 months, or occasionally even for six months or more, at least after prolactin and testosterone in the serum have returned to normal level. Men who suffer from hypogonadism following diseases in the hypothalamus or in the pituitary gland, can be treated with gonadotrophins. The treatment is administered with HCG 1500-2000 IU three times a week by subcutaneous or intramuscular injection for a minimum of six months, HCG having biological activity like LH. The HCG dosage is supposed to be adjusted according to symptoms of hypogonadism, concentration of testosterone in the blood and perm parameters. Some patients with acquired hypogonadism can be treated with HCG only, in order to produce enough sperm, however, should a patient, after 6-9 months, still have severe azoospermia or oligospermia, treatment with HMG or FSH recombinants should be added as well (Wang \& Swerdloff, 2016).

In case of varicocele - there are contradictive data regarding the efficiency of repairing varicocele, therefore, an operation to repair varicocele is not recommended routinely amongst couples with infertility, although it might help men with a level three varicocele. Additionally, this solution can be suitable for relatively young people with a short duration of infertility (Wang \& Swerdloff, 2016).

Empiric treatment - many a treatment is performed empirically for male fertility, including aromatase inhibitors as well as other hormones and additionally, administering vitamins. As a principle, a recommendation is given to infertile men to wear boxer shorts instead of tight underwear and not to take a shower or baths with boiling hot water from the rationale that high temperature in testicles can damage sperm production (Wang \& Swerdloff, 2016). 
Occasionally, ovulation induction is combined with intra-uterine insemination in cases where ovulation problems exist, combined with a male factor which causes fertility problems in spouses (Fauser, 2016).

\subsection{Intra-Uterine Insemination - IUI}

Intra-uterine insemination is a process in which processed and concentrated sperm is inserted directly into the uterine cavity. Clinical use of IUI is based upon the hypothesis that placing great number of sperm cells inside the uterine cavity improves the chance of conceiving. IUI is particularly useful for couples with disorders in sexual performance (Vaginismus or ejaculation problems), or when someone is a carrier of diseases transferred by sexual contact, in cases of cervix problems or infertility of light male origin. IUI enables the sperm to bypass hostile cervix factors and thus increasing the number of sperm cells entering the uterine cavity.

For women who have undergone ovulation induction, including those with unexplained infertility problems or light or mild endometriosis, pregnancy rates are considered higher when IUI is used as complementary procedure instead of timed intercourse. In those couples, IUI is used as treatment of a basic and economical level, before reaching in vitro fertilization. Pregnancy rates after IUI are dependent upon male and female factors and technical factors. Intra-uterine insemination is usually done from fresh sperm of a spouse. The sample is collected to a sterile cup in order to minimize the risk for uterine infection from pollutants.

It is advised to avoid use of lubricants which are usually poisonous to sperm. In the process of sperm preparation, it is required to separate the sperm cells from excretions containing prostaglandins and from the seminal fluid, as it can cause contractions in the uterus and seldom an anaphylactic response should the sperm be injected as-is directly to the uterine cavity. Additionally, sperm enhancement results in maximal number of motile sperm cells, removes cellular waist and brings about concentration of the sample (Ginsburg, 2016).

IUI is performed as treating a case of retrograde ejaculation in which a spouse's sperm is collected after a patient takes bicarbonate in order to neutralize urine PH. Due to the fact that without neutralization, most of the sperm or all of it can die upon entering the urinary bladder. Additionally, instruction is given to drink water to dilute the urine for decreasing the intense effect of urine upon the sperm. The spouse receives two cups: initially he should urinate before the sample, afterwards, sperm is collected to a sterile cup and immediately afterwards he should urinate into the second cup (medicine.missuri.edu/fertility). 
There are two techniques for semen enhancement: swim up and use of density gradient centrifugation. A systematic review of random studies comparing these two techniques has not revealed any statistically significant difference in pregnancy rates, however, the percentage of motile sperms cells that recovered after enhancement is higher in gradient technique than in swim up technique, therefore, for men with a small quantity of sperm cells or with a low motility sperm, this technique is preferable. The ovum can be fertilized up to 24 hours after ovulation and sperm can fertilize up to 48 hours after entering the female genitourinary system. The timing of IUI is based on combination of those facts. The question whether to do one or two IUIs is controversial. In last two random studies and a meta-analysis from 2010, it was found that pregnancy rate was similar whether one IUI was performed or whether two were performed. Out of this reason, IUI is performed one day after the LH level is at its peak (Ginsburg, 2016).

Natural cycles (without ovarian stimulation) are suitable for patients with ejaculation problems, vaginismus, or infertility due to cervix factors, usually causing ovulation before the IUI whereas a controlled ovarian stimulation is common with IUI for fertility treatments in couples with unexplained infertility problems. In four random studies of patients with unexplained infertility, pregnancy rates were higher when IUI was performed in a cycle that in which ovarian stimulation was induced than in natural treatment cycles - 25\% as compared to $14 \%$ (Ginsburg, 2016).

Ovulation with HCG - IUI is performed 36 hours after injection of HCG. The minimal size of 15-23 mm of the leading follicle, prior to injecting HCG is controversial. For those women who received ovarian stimulation with Clomiphene, pregnancy rates were higher when IUI was performed after spontaneous ovulation based on LH levels as compared to IUI after injecting HCG to induce ovulation (Ginsburg, 2016).

\subsubsection{IUI procedure}

Prior to performing IUI, a correct identification of both patient and sperm sample must be verified in the clinic lab. The processed sperm is extracted with the medium solution into a syringe, the syringe is connected to $18 \mathrm{~mm}$ polyethylene catheter intended for insemination (there are two types of catheters, one is soft and flexible which can be bended - Kremer, and the other is inflexible and curved - Cook. It is preferable to use soft and flexible catheter which can be adjusted to the curve of a patient's uterus and it is less traumatic to the endometrium. If 
there is any difficulty to go through the internal- Os with the catheter, it is possible to perform the procedure under abdominal ultrasound and instruct the patient to a full bladder.

As compared to these, inflexible catheters are more easily inserted into the uterine cavity as they do not bend, however they cause discomfort to a patient and are more traumatic to the endometrium and thus they are associated to more cases of vaginal bleeding. After injecting the sperm, the patient is asked to lie on her back or in a Trendelenburg position for 10 minutes, despite the fact that there is limited available data regarding the question whether rest has some value in improving pregnancy rates.

After stay in the clinic, a patient can return to her normal routine activity. It must be emphasized that wetness is possible after the procedure and it stems from watery excretions of the cervical mucus - this does not mean that the sperm sample has come out, there might be contractions and a sensation of discomfort in the abdomen. Painkillers can be taken if necessary, additionally, slight bleeding or blood stains are a possibility. Additionally, it is possible to have intercourse after IUI should the couple want it. About two weeks after the IUI, blood/urine test for pregnancy should be done should there be no menstruation. However rare but still possible complication is called PID - Pelvic Inflammatory Disease. It is not clear whether the chance for ectopic pregnancy is higher for a woman who has undergone IUI with identical risk factors to a woman who conceived without IUI (Ginsburg, 2016).

\subsubsection{Donor Insemination}

In the past, insemination from sperm donation was mainly in cases of infertility of a male factor. However, over the years, the indications for IUI from a donor have expanded and it has become an alternative approach for some women. The indications are:

- Male spouse infertility - when the male spouse has azoospermia, severe minority of sperm cells or other severe deficiencies in the sperm causing infertility.

- Couples in which one or both spouses are carriers of hereditary disease - when there is a potential of hereditary disease with the offspring, sperm donation is to be considered in couples in which one or both spouses have a disease or both are carriers and they can give birth to a damaged child. According to type of genetic disorder, these couples can prefer IUI from sperm donor who is not a carrier (or to perform PGD).

- A single woman without a male spouse and lesbian couples - ASRM - the ethics committee of the American Society for Reproductive Medicine approved the rights of women - both single and lesbian, to gain access to fertility services. Insemination from 
sperm donation is usually used for achieving pregnancy amongst women without a spouse such as single or lesbian women.

- In couples that have no match in the red blood cells antigens (for example D or KELL) which are related to a Hemolytic Anemia disease of the newborn or a history of irreparably damaged baby.

Counterindications: women with fertility problems due to Fallopian tubes factors, uterus deficiencies, active pelvic inflammation or women who do not ovulate do not constitute good candidates for sperm donation insemination (Ginsburg \& Srouji, 2016).

\subsection{Sperm bank}

Sperm bank is an entity that was established in order to assist women and men to give birth to a child when the "natural" way fails. Sperm bank is a pool of sperm donations. Currently, in Israel, 17 sperm banks operate, some are public, operating in hospitals and a small number of private banks. Sperm bank constitutes a connecting link between women or couples who need sperm donation and men who give sperm donation (Fertility and fertilization, a complete guide, 2011).

Instructions of ASRM from 2013 regarding donation of sperm and embryos provide detailed and updated recommendations for evaluation of potential sperm and ovum donors in combination with an updated information regarding survey and blood tests for diseases transferable by sexual intercourse (including HIV), genetic diseases and psychologic evaluation. Sperm donors are to be of legal age (above 18), however preferably under 40 . However, many a sperm bank limits its donors' age under 35. The top age limit was designed to limit the possibility of genetic mutations related to parents' age, despite the contradicting data. Psychologic evaluation by a professional in the field of mental health should be given to all donors.

Emotional, psychological, and social aspects of sperm donors constitute part of this evaluation. Donors are as well required to fill questionnaires regarding their viewpoints or concerns regarding transfer of information for future contact with the biological offspring. Donors are limited to create 25 births amongst population of 800,000 people in order to minimize the chance of unintentional blood relation, however instructions and limitations differ between countries due to differences in size of population and social and psychological influence (Ginsburg \& Srouji, 2016). Most of Israeli donors are young, mostly students, soldiers, or those after their military service. The minority are older men. 
Additional cases in which sperm preservation is performed, are in cases that men preserve their sperm cells in cryopreservation should they know that they have a medical disease such as: cancer or amputation of testicles that in the future might prevent them from having children. The cryopreserved sperm dose will serve the specific man in the future and in any case, is not used for sperm donation. The Ministry of Health determined several criteria for potential sperm donors. It was done for guarantying the quality of donations. Basically, from 10 potential donors only 3 (or less) will be approved (Fertility and fertilization, the complete guide, 2011).

\subsubsection{Bill of sperm donation regularization in Israel}

In October 2016, the Ministry of Health promotes a comprehensive bill to regularize sperm donation in Israel and to operate sperm banks in the State (in addition to those existing). The new law is expected to limit the number of women whom a man can donate to, to just nine. Until present time, sperm donation array was conducted according to nation health regulations and instructions of the Attorney General, however, the process was yet not anchored in main legislation. Sperm banks in various hospitals might limit the number of women receiving donation from a single donor, however, there is no coordination between the banks.

As there is no central registration showing were each man donated his sperm and in Israel there is no limit to the amount of the sperm donations one can donate, a need arises for establishment of national information center that will be managed by the State and this step will enable children to avoid incest. A pool will be activated which will enable a person to find out whether he was born from a sperm donation, while preserving confidentiality of the donor and will enable the spouses to find out before their marriage whether they are related. The law as well proposes enabling a sperm donor to withdraw his consent, as long as the sperm has not yet been used in fertilization. Should a sperm donor change his mind, the bank manager can demand the donor to refund the financial compensation received. Additionally, the bill might put limitations upon unmarried couples, which might create multiple difficulties in the future for lesbians and single mothers (Health News, 2016).

It is important to indicate that in Israel, there is a constant shortage of sperm donations and following that many a woman turns to importing sperm donations from abroad. Many a bank enables a woman to purchase sperm donation from abroad, however, it must be indicated that sperm imported from abroad is more expensive due to the fact that it should be delivered from abroad in special containers (Fertility and fertilization, the complete guide, 2011). 
A sperm donation cost can change according to a sperm bank and based upon how the sperm was prepared (washed compared to unwashed) and according to characteristics of a donor (level of sample). Typical prices of unwashed sperm can span between $180 \$$ and $500 \$$, as compared to between $250 \$$ and $700 \$$ for washed sperm. These prices do not include storage, transport, routine evaluation and blood tests or payment to a doctor for performing the insemination (Ginsburg \& Srouji, 2016). In Israel, a woman interested in sperm donation, purchases the sperm doses by herself. In the public sector, the price of sperm dose is 350 NIS, sperm wash 300 NIS and a doctor's consultation and insemination - 350 NIS (given free of charge by a patient's HMO with form 17). Full price: 1,000 NIS (not including tests) and after a discount with form $17-650$ NIS, whereas in the private sector, the payment is a little higher and might reach 1,200 NIS.

When a sperm donation is foreign, the price can span between 2,500 and 4,500 NIS and even higher for a sperm dose, this cost depending upon several criteria: quantity of purchased sperm donations, type of sperm doses (washed or unwashed), quality of dose - depends upon results of tests performed on the sperm sample and the donor and type of sperm donor - for example, if the donor possesses specific qualities which are highly demanded (Fertilization and Fertility the complete guide, 2011).

\subsubsection{Selecting a donor}

Sperm banks offer different types of information regarding their donors, information types given include donors' profiles (race, height, weight, hair, skin and eye color, level of education, religion and ethnic background), audio recordings, hobbies, their baby photos, personal profiles, reports of impression of the staff, papers written by the donor, reports of facial features and history of previous success in producing pregnancy. This information can be free of charge, however, there are banks that require payment for more detailed descriptions or photos. There

are some banks that even offer consultation service for matching a donor (Ginsburg \& Srouji, 2016).

In order to improve pregnancy rates, the focus is on: number of inseminations, timing of insemination and methods of insemination. The primary variable which is not changeable and which reduces the chance of successful outcome of the procedure includes old age of a mother, as age of mother fills a key role in determining fertility of a woman regardless any measures taken for achieving pregnancy. Effect of advanced age on success of insemination was illustrated in review of accumulating pregnancy rates in women above and below the age of 30 
and included details from about 3,000 cycles in a span of 10 years. Women below 30 had higher pregnancy rates in three, six and 12 months than women above $30(21 \%, 40 \%$ and $62 \%$ as compared with $17 \%, 26 \%$ and $44 \%$, respectively).

Some claim that cryopreservation of sperm and defrosting thereof later, reduces its motility and vitality, which appears to cause low pregnancy rates. A random crossed study that was conducted, demonstrated low fertility with cryopreserved sperm as compared with fresh one 381 patients were examined in the study who have done alternate cycles of fresh sperm as compared to cryopreserved sperm which was defrosted, fresh and cryopreserved sperm cycles gave fertility of $18.9 \%$ as compared to $5 \%$ respectively. New evidence indicates that long-term sperm storage in cryopreservation does not affect sperm motility which was perceived as a predictor of sperm functioning after defrosting (Ginsburg \& Srouji, 2016).

A control study data demonstrates that the amount of fresh sperm injected in IUI process must be above critical limit in order to achieve maximal pregnancy rates after low pregnancy rates were observed of women after insemination with cryopreserved sperm, and therefore, data of Denmark Central Sperm Bank ( $n=3,418$ cryopreserved doses) revealed that increasing the quantity of sperm in general motility from $10 \times 10^{6}$ to $11 \times 10^{6}$ to $19 \times 10^{6}$, the chance to achieve pregnancy increased, however, as soon as the quantity was above $19 \times 10^{6}$, no further advantage was observed. Most centers make an effort to select donors with consistently high sperm concentration in their sample and many donors are rejected due to inadequate sperm count.

There is no data from random studies in comparison of success rates of one insemination as compared with two inseminations in each cycle, in women who received cryopreserved sperm donation. However, two observational studies revealed an increase in fertility when two consequential inseminations were performed in middle of a cycle. Out of this reason, inseminations are performed on a day the $\mathrm{LH}$ level is at its peak and on the following day (Ginsburg \& Srouji, 2016).

\subsubsection{Ethical problems}

In Israel, sperm donation is allowed only in anonymous route. The public committee for examination of fertility and birth subjects headed by Prof. Mor-Yosef, recommended establishing additional non-anonymous route. The existence of the two routes supplies a response to a concern of reduction of number of donors should only identified donation be possible. Furthermore. the committee determined that despite the fact that it is a right of a person 
to discover his roots, there is justification for continuation of existence or nonexistence of a father who takes part in their life. In recent years, support of revealing the fact of contribution to an offspring intensifies, as well as a trend to enable access to non-identified information regarding donors to an offspring who wishes to know about his origin (Ethics Committee, 2015).

Other ethical dilemmas include the idea of whether or not it should be obligated that identity of a donor is available in order to enable later contact (medical or according to need) and whether or not parents are required to reveal to their child the way they were conceived. In USA, the ASRM ethics committee highly recommends revealing success of conceiving to donors, however it admits that parents who were donation recipients have the right to make that decision. The number of programs proposing donors an open identity in USA is increasing and the ratio between open identity of donors and anonymous donors tends to increase over time in programs that offer this service (Ginsburg \& Srouji, 2016).

Revealing to offspring born from donation occurs earlier amongst lesbian couples and single mothers as compared to heterosexual couples. Even those heterosexual couples who expressed intention to reveal their offspring information regarding the donor, less than $5 \%$ did so by the time their child was 3 years old. A study regarding offspring's of donors revealed that late revelation is more correlated to negative emotions towards their donor. In Sweden, when legislation that was passes obligated the identity of a donor to be maintained and available for an offspring's request upon their reaching maturity, recruitment of donors dropped only temporarily after the new regulations came in force (Ginsburg \& Srouji, 2016).

\subsection{Number of treatment cycles before transition to IVF}

Women who have undergone insemination from a donor without fertility diagnosis can continue inseminations in natural cycles. After 3-6 natural cycles a transition to treatment with ovulation induction with Clomiphene or gonadotrophins. After 3-4 cycles of ovulation induction (depending on age of a woman) without achieving pregnancy, IVF - In Vitro Fertilization treatment should be considered (Ginsburg \& Srouji, 2016).

As for couples in fertility treatments, without absolute obstacles preventing conceiving (bilateral obstruction) of the Fallopian tubes or severe sperm problem) couples are offered 3-6 treatment cycles of ovulation induction with IUI before moving to IVF treatments. For young couples without a clear reason for a problem of conceiving, a total of one year of unprotected sexual intercourse and number of conventional treatment cycles are offered as chances of 
conceiving are reasonable during this period (about $85 \%$ will conceive in the course of these treatments).

As for older couples, the trial is for a shorter period of time as for them, conventional treatment is less successful, and time has a bigger role in chances of conceiving. It is not improbable to offer IVF as initial treatment possibility for couples when the female spouse is above 40 (Paulson, 2016).

\subsection{IVF (In Vitro Fertilization)}

In Vitro Fertilization is one of the most common treatments amongst the assisted reproductive technology (ART) treatments. IVF is the most common treatment technique in modern age, in this process, an initiative encounter occurs between an ovum and a spermatozoon in laboratory conditions, as an alternative for their natural place of encounter - the Fallopian tube in a woman's body. Later, one embryo or more are inserted to the uterine cavity while administering giving a supportive hormonal treatment. This procedure takes place in a time space of two weeks and is called - IVF cycle. Originally, this treatment was invented in order to bypass the need for Fallopian tubes for women whose Fallopian tubes are missing or blocked (Ministry of Health website). However, in recent years, the indications were extended significantly since the first IVF that suppled a response for Fallopian tube factor and led to pregnancy and the birth of Louise Brown. Since then, there has been much increase in demand for ART which was greatly affected by a changing socio-economic dynamic of our society. The estimation is that almost $50 \%$ of couples with continuous fertility problems, or who are in treatment for more than two years will eventually need a referral for IVF treatment (Mahmoud et al., 2013).

\subsubsection{IVF indications}

In a paper by Paulson (2016), IVF is the first treatment line when there is complete obstruction in both Fallopian tubes, however, it became available for women with other causes for infertility, including one or more of the following:

- Severe infertility of male factor - (light male factor can be treated with insemination) when it is Azoospermia, or one or a combination of the following in more than one sample, in a gap of three months from one another: severe minority of sperm, (less than $10 \times 10^{6}$ ), low sperm motility (less than $40 \%$ motility) or damaged morphology.

- Low ovary reserve (when time of conceiving is crucial and chances of success with other treatments are low). 
- All other reasons for infertility after treatment failure with less invasive treatments (problems with ovulation, Endometriosis and unexplained infertility).

- Ovary failure.

- Cause of uterine source (such as Asherman's Syndrome or irreversible deformation of uterine cavity).

- Choosing gender of a newborn - for couples interested in offspring of a gender (male or female) without having a medical indication for this.

- Preimplantation Genetic Diagnosis - PGD.

- For woman born without uterus or woman that medically cannot conceive (IVF for surrogacy).

And in a case of preserving fertility be it for medical reasons (women with decreased response, severe endometriosis, women suffering from autoimmune diseases or women before ovary surgery), or from non-medical reasons (women interested in preserving eggs who are above 30 but below 41 years of age) for future use (Ministry of Health website).

Medical Administration Circular of Ministry of Health 2014: according to State Health Law, In Vitro Fertilization treatments will be financed by HMOs for couples who do not have children in their current marriage (up to two children), and as well for a woman without children who wishes to start a family headed by an independent parent (single-parent family) for giving birth to first and second child. The funding is awarded with no bearing to financial situation, emotional state, or education level of those wishing to become parents (Hashiloni-Dolev, 2013). Following are recommendations of National Council for Gynecology, Neonatology and Genetics for several treatment cycles:

- IVF treatments are given to women as early as of the age 18 and maximal age for treatments is 45 (until the $45^{\text {th }}$ birthday).

- For women who are already 39 years of age, it is possible out of medical considerations to perform IVF treatments as a first option for fertility treatments.

- For women above 42 years of age, not more than three consecutive IVF treatment cycles will be performed, in which a stage of embryo transfer has not been achieved (not including cryopreserved embryo transfer from previous treatments). 
- In any age, after four consecutive treatment cycles, in which a stage of embryo transfer has not been achieved, or after eight IVF cycles without clinical pregnancy, there will be a discussion of treating staff and program for further treatment, including IVF treatments will be determined according to the conclusions of the discussion.

According to Fertility and Sterility report, Israel provides optimal usage of $100 \%$ of IVF treatments, meaning, the highest number of IVF cycles provided by the healthcare system. Since 1990, the rate of IVF cycles in Israel has almost doubled amongst women in their fertility years. According to Ministry of Health, a rise in IVF treatments is higher than rate of population increase. In 2010, there were 17.8 cycles for 1,000 women (ages 18-49) as opposed to 12 cycles in 2000 and 8 cycles in 1995. In 2010, 4.2\% of all live births in Israel were following IVF treatments as compared to $2.6 \%$ in 2000 . The rate of live births stems from an increase in number of treatment cycles in women, however, the average success rate of IVF remained fixed over recent decade, about 15-17\% live births per treatment cycle (Simonstein et al., 2014).

\subsubsection{Advantages and shortcomings of IVF treatment}

In a paper by Kuohung and Hornstein, 2016, IVF treatment indeed has its advantages, however, it as well conceals within itself some shortcomings. The advantages are:

- Better success rate for each treatment cycle than other fertility treatments

- Less invasive procedure than Fallopian tubes operation.

- Ability to overcome other infertility factors should these exist (male factor, cervix factor, low ovarian reserve).

- Location and extent of Fallopian tube damage has no effect on the outcome.

Shortcomings:

- High cost of each treatment cycle and multiple treatment cycles might be required

- There is a need for IVF each time pregnancy is desired

- Requires frequent injections and monitoring

- Raises risk of multi-fetal pregnancy

- Raises risk of ovarian hyper-stimulation.

- There might possibly be there a little higher risk of complications around birth than in natural pregnancy

\subsubsection{Factors affecting success of IVF}

There are several factors that can affect the success of IVF (age, diagnosis of infertility, obstetric history), however, a woman's age is the main factor determining success of IVF treatments. 
Even though IVF might overcome fertility problems, especially amongst young women, it cannot "rectify" age-dependent fertility drops amongst older women, particularly those above 40 years of age. In 2012, the percentage of treatment cycles with fresh embryos from an egg of a woman that resulted in live birth, according to a mother's age was as follows: under 35 $40.7 \%, 35-37-31.3 \%, 38-40-22.2 \%, 41-42-11.8 \%$ and above $42-3.9 \%$. The top limit of age for performing IVF (from a woman's egg) is controversial and changes according to location, however it usually spans between 41-45 years (Paulson, 2016).

Additional factors:

- Ovary response (number and quality of the eggs) - the second most important decisive factor of rate of success of live birth after a woman's age. Low ovary reserve has lesser results than women in same age with normal ovary reserve as a response to ovarian stimulation.

- Infertility duration - there is opposite ratio between duration of infertility and chances of success after IVF. Fertility duration of six years or more, seems as predicting a bad outcome continuously.

- Number of unsuccessful previous treatment cycles - the chance of live birth decreases as the number of unsuccessful treatment cycles increases and begins to decline rapidly after four unsuccessful treatment cycles, however, it does not affect rate of spontaneous pregnancy (Mahmoud et al., 2013).

- Hydrosalpinx - caused by damage or obstruction in end of the Fallopian tube which causes the Fallopian tube to be filled with fluid. Hydrosalpings can be caused by old infection in the Fallopian tubes occasionally due to STD, other factors include: previous operations (especially Fallopian tube operations), severe pelvic adhesions, endometriosis or other indications of infection such as appendicitis (Reproductivefacts.org). $10-30 \%$ of women treated with IVF are found to have unilateral or bilateral hydrosalpings, the diagnosis is done by laparoscopy which is the gold standard, uterine x-ray has sensitivity of $65 \%$ and specificity of $83 \%$ as compared to laparoscopy (Loutradis, 2011). Studies consistently reveal that presence of hydrosalpings is related to low IVF results, moreover, random studies proved that Fallopian tubes amputation prior to IVF treatment in women with hydrosalpings improved pregnancy rate (Paulson, 2016).

- Smoking can as well affect success of IVF (less eggs develop). 
- Obesity - infertility is more common amongst overweight or obese women (BMI 25-29 or above 30) and IVF might be less successful in such women. In a retrospective study of almost 500,000 spontaneous IVF cycles, it was found that in obese women, there is a drop of $6 \%$ in rate of intrauterine pregnancy and a drop of $13 \%$ in rate of live birth as compared with women of a proper weight (Paulson, 2016).

\subsubsection{Risks of IVF treatment}

Controlled ovarian stimulation has negative implications and risks which include:

- Ovarian hyper-stimulation OHSS - is rare however a severe complication related to Controlled Ovarian stimulation- COS. Medium to severe OHSS occurs in about 1-5\% of treatment cycles. The syndrome includes a wide variety of findings, such as: ovarian enlargement, edema, blood concentration, Thrombophilia, and electrolyte imbalance. Sever OHSS can cause serious complications, such as: Pleural Effusion, acute kidney failure and venous thrombosis (ASRM, 2016). In a study by Toftager et al. from 2016, instance of medium OHSS in an antagonist cycle is about $5.1 \%$ as opposed to $8.9 \%$ in an agonist cycle. An instance of hospitalization is about $1.7 \%$ as opposed to $3.6 \%$ accordingly.

- Multi-fetal pregnancy - occurs in about $30 \%$ of all pregnancies in patients who have undergone ovarian stimulation and this phenomenon is correlated to an increase in risk of pregnancy loss, birth complications, premature babies and illness of long-term damage to the newborn (Elnashar, 2012).

- Ovarian torsion - is an acute abdominal condition requiring immediate intervention. After IVF treatment, ovarian hyper-stimulation might increase the ovaries and thus cause for their torsion. Its incidence is above $0.1 \%$ of total IVF treatments (Tsai et al., 2015).

- Extrauterine pregnancy - rate of instance of extrauterine pregnancy after IVF treatment with embryos transfer is $2.1-8.6 \%$ of total clinical pregnancies, which is higher than spontaneous pregnancies $-2 \%$ (Cheng et al., 2015).

- Ovarian cancer - there are few reports discussing an increase of incidence of ovarian cancer after treatment with ovulation elements over a long period of time, however, those reports were not confirmed in additional studies and information is still being collected conclusions thereof will be consolidated in several more years. It is understood 
that as number of in-vitro fertilization treatments rises, thus a patient is exposed to a higher risk (Asaf Harofe Hospital website).

Additional complications of IVF treatments according to a paper of Mahmoud et al. from 2013, include:

- Allergic response to injections of the treatments itself, be it Gonadotrophins or agonist or antagonist medical drugs.

- Bleeding during egg retrieval from the cervix, the ovaries, or in rare cases from blood vessel in the pelvis.

- Infection created mainly from inserting a needle through endometrium.

\subsubsection{Egg retrieval procedure}

After administering ovarian stimulation with Gonadotrophins, monitoring the response to stimulation is performed by ultrasound and level of hormones in blood $(\mathrm{E} 2+\mathrm{P}+\mathrm{LH})$. When hormone level reaches the expected levels and when there are minimally 3 follicles that are bigger than $17 \mathrm{~mm}$, the final maturation of the ovum is brought about by HCG injection. Egg retrieval is planned for about 36 hours after administering the HCG (to prevent ovulation which occurs 40 hours after injection of $\mathrm{HCG}$ ). The procedure is performed in operating room under short-term general anesthesia, the egg retrieval is performed under ultra-sound machine through vaginal transducer equipped with a needle, the eggs are received by puncturing the follicles and extraction of the fluid (Cauffman - global fertility academy website) There are few complications after this procedure: infection and bleeding. Pelvic inflammation is rare $-3 \%$ and passes after administering antibiotics. This risk reaches $0 \%$ should preventive-prophylactic antibiotics be administered (Paulson, 2016).

\subsubsection{Cryopreserved embryo transfer}

Cryopreserved embryo transfer can be performed through different treatment cycles including natural ovulation period and artificial period in which the endometrium is prepared by administering estrogen. The transfer timing is dependent upon development of the endometrium. In natural period, conceiving of the uterus is monitored by ultrasound and the transfer is planned about 3-5 days after LH increase in blood serum (meaning, ovulation) and dependent upon an embryo's stage ( $3^{\text {rd }}$ or $5^{\text {th }}$ day) and support is given in the luteal phase in day of transfer through progesterone for two weeks. Opposite to that, an artificial period is used in patients with irregular periods who do not ovulate. Development of the endometrium is 
dependent upon administering estrogen PO from second day to the menstrual period and monitoring its development is done by ultrasound until the endometrium achieves $8 \mathrm{~mm}$ and then the transfer is executed. Administering hormonal support with progesterone begins about 3-5 days before the transfer and continues to the $9^{\text {th }}-12^{\text {th }}$ week of the pregnancy. Natural and artificial periods are equally efficient from an aspect of clinical pregnancy results and that is according to a study conducted in 2010, which included 22 random studies, compares methods of endometrium preparation (artificial/natural) and additionally, in 2013, a meta-analysis including 20 papers was published and no evidence was found that one method of endometrium preparation is better than another (Pelkonen, 2016).

\subsection{Egg donation}

IVF is more effective in advanced age, however, there are low rates of success amongst women of age 40 and above, usually less than $20 \%$ per treatment cycle. Women above age 42 , who did not manage to conceive with other treatments (including IVF) or with premature ovarian failure known as early menopause, treatment possibilities for them are limited.

The only alternative they are facing is egg donation from a woman in her 20's or early 30's. High success rate of conceiving from egg donation supports the fact that the quality of an egg is related to age which constitutes the main obstacle to pregnancy amongst older women. Due to low egg quality, there are less chances of conceiving and more chances of miscarriages.

Significant change in egg quality is in the frequency of genetic defects that is termed aneuploidy (too many or too little chromosomes in an egg). The older a woman is, more and more eggs have too many or too few chromosomes and the meaning of that is that should fertilization occur, the embryo would have too many or too few chromosomes. Most of these embryos do not end up in pregnancy at all or they cause miscarriage. This reason explains the low chances of getting pregnant and high chances of miscarriages amongst older women. Although the chance of successful pregnancy in older age is considerably higher in an IVF cycle with egg donation, many a couple or single woman in their 40's would prefer the low chance of pregnancy and use their own eggs.

At 43, the chance of getting pregnant through IVF is less than 5\% and at 45 the only probable treatment option is through egg donation. In a cycle of egg donation - the egg donor receives treatment with fertility medical drugs in order to stimulate production of multiple eggs in the ovaries, and at the same time, the donation recipient receives hormonal treatment in order to prepare her uterus to receive the fertilized eggs (embryos). After egg retrieval from the donor 
they are fertilized in a lab with sperm of the spouse of the donation recipient while administering supporting hormonal treatment. The remaining embryos can be cryopreserved for future cycle. Although IVF with egg donation offers a woman in advanced age to experience pregnancy, delivery and motherhood, however, the child is not genetically related to the mother although being genetically related to the father and the donor. Many programs recommend consultation so that all those involved in the egg donation understand the ethical, legal, psychological and social implications involved (ASRM, 2012).

\section{Fertility difficulties, a psychological and emotional aspect}

\subsection{What is stress?}

According to a book by Folkman, "Appraisal and coping", from 2013 - stress is traditionally defined as stimulation, that often called a stressful factor that happens to a person as a response characterized by a negative psychological stimulation, particularly, anxiety. The cognitiverelative theory of Lazaros defines stress as a specific relationship between a person and the environment, which the person evaluates as burdensome or surpassing his resources and which jeopardizes his wellbeing. The evaluations are determined simultaneously by perceptions of environmental demands and personal resources, they can change over time due to efficiency of coping, change of demands or improvements in personal abilities.

Stress arises when there is unsuitability between physical or psychological demands of a situation and resources of biological, psychological, or social systems thereof (Mcleod, 2015).

\subsection{Infertility and treatment as stress factors}

According to a paper by Ginor, from 2015, in every civilization, parenting is considered as one of the main roles in lives of the adult person. Infertility, according to Man's development theory of Arikson, might cause damage to mental development of an adult - in ability of creation of intimate relations and ability of creativity (generativity). Since parenting is an important component in identity of an adult person, response of people to discovery of infertility is often involved with a feeling of despair.

Fertility treatments that offer more and more people a possibility of becoming parents are involved in complex mental coping: they are demanding and difficult, complex, and cumbersome for execution, damaging for self-image, spouse relations and the fabric of life. This crisis is called in literature - Reproductive Trauma (Kaplan, 2015) 
Infertility experience is usually connected to unexpected pressures that can influence the personal life of a person, the social support system and marriage relations. Those pressures can bring about significant disruptions in life of a person and be related to intensified psychological distress in both women and men. One of main distress symptoms reported amongst fertility patients is depression (Brennan et al., 2014). Compared with patients with other medical conditions, psychological symptoms related with infertility are like those related with cancer, blood pressure and heart diseases.

Many feel that reproduction is their basic nature and consider it to be a basic function of life. Most people, naturally, take for granted the ability to conceive in any time in their life they are ready. When this event does not take place, many of a couple feel sensations of great stress and distress, anxiety, anger, cognitive damage and feelings of non-attraction, there is no doubt that patients understand that fertility treatments constitute a challenge, both physical and psychological. Through an easy access to information through thousands of websites focusing on meticulous fertility treatments and stories and complaints of acquaintances, most of infertile women are expected to have a negative impression regarding the treatment even prior to consulting with a doctor (Domar et al., 2012).

Yaun (2013) found that In Vitro Fertilization is stressful psychologically and emotionally, the tension of before, during and after an IVF treatment is multi-dimensional. There is a chronic stress source caused by a constant threat of infertility and lose of hope. Another origin of stress is a threat of the treatment itself: daily injections, drawing blood, ultrasound, egg retrieval and a possibility of failure in each of the various stages. The third origin of stress is the possibility of spontaneous miscarriage. Egg retrieval and results of pregnancy tests proved that these constitute the most stressful stages in an IVF cycle.

Patients who have difficulty in accept their infertility and who have no children, tend more to experience anxiety and depression when they are notified that a treatment has failed. And patients who experience helplessness regarding infertility and the treatment accompanying it, tend more to experience anxiety and depression when they are notified that a treatment has failed. In couples, the way one spouse responds to infertility and the treatment accompanying it, is related to the way the other spouse responds (Gameiro, 2015).

\subsection{Emotional distress of fertility treatments}

In a paper by Domar (2015), the belief that psychological factors play a role in infertility is years long and there are evidences that high stress levels might influence the results of fertility 
treatments as well as contribute to decision of patients to continue a treatment. Psychological implication of fertility treatments is high and one of the factors causing it is that result is unclear. Furthermore, mental distress is connected to failure of fertility treatments and interventions in order to relieve the pressure relate to rise in pregnancy rates. Additionally, tension before conceiving might increase a risk of infertility. Psychological pressure seems to be more common in a spouse who has fertility problem. Incidence of psychological stress in female fertility patients was demonstrated in a study conducted by a psychiatrist who held personal interviews amongst 112 barren women who visited a fertility clinic prior to medical assessment - $40 \%$ of the patients fitted criterions of a psychiatric disorder, when the most common diagnosis was an anxiety disorder (23\%) and next to it, a depression disorder (17\%).

The first response for acknowledging that it is infertility, is usually of amazement. Following it, appear feelings of: distress, anxiety, guilt and depression and sometimes a feeling of loss. Loss of ability to bring a baby to the world in a natural way, a feeling that the medical processes are alienated, lacking intimacy that take control over life. All these might damage self-image of a man and a woman as a person who is healthy, creative, and human. Unique and different sense of grief might be added to all these, as discovery of infertility is gradual, therefore, a feeling of grief that is derived from it is not finite. After a strong emotional response which can as well can be experienced as a crisis, usually comes a period of acceptance and recognition. This period can be characterized by sharp ups and downs in mood. Hope for success of fertility treatments bring along with it encouragement and happiness, however, these sensations can be quickly changed with bitter disappointment and despair facing failure of the treatment (Ginor, 2012).

In an article by El-Kissi from 2013, it was found that $87.5 \%$ of infertile couples reported that finding out regarding infertility is tiresome with no significant differences between men and women. A number of studies focused on the nature of the stressful and demanding fertility treatments. Physically, some procedures cause pain such as: egg retrieval or testicle biopsy, others, like hormonal treatments, cause side effects for both men and women. Furthermore, fertility treatments are experienced as a tense atmosphere with fear of failure in each attempt. A couple attempting to conceive will therefore feel frustration and uncertainty.

Psychological pressure leads to imbalance of the endocrine mechanism and contributes to infertility. In men, it is expressed in decreased testosterone in the serum and thus might damage 
sperm quality by reducing sperm amount, motility and number of morphologically improper sperm cells (Bnongade et al., 2015).

Stress and anxiety are higher when patients are expecting results (for example, a waiting period before pregnancy test, before eggs retrieval and embryo transfer). Patients experience high emotional distress when they are being informed that a treatment did not succeed. 1-2 women out of 10 experience significant clinical levels of depression symptoms (Gameiro, 2015).

Studies conducted around the world, found correlation between stress levels of women prior to or upon beginning of an IVF treatment cycle. Majority of studies reveled that high levels of stress are indeed correlated with low success levels of IVF, meaning, decrease in pregnancy rates (Hagela et al., 2016).

Studies reveal that the main sources of pressure of fertility patients are impact of infertility upon their social lives, their social health and their relationship with their spouses. Stress level in fertility patients tends to grow as the treatment is more intensive and as the duration of treatment prolongs. Therefore, IVF patients are expected to experience more pressure than patients who are in early stages of their infertility assessment. Additionally, medical drugs that are employed for treating infertility might contribute to depression symptoms. An observational study of women receiving GnRH Agonist found that $75 \%$ had depression symptoms.

Many an IVF patient reports depression symptoms even prior to beginning of their treatment cycle, which probably reflects the implications of recurrent less invasive treatments with no success. Most IVF patients report that the treatment is more stressful psychologically than physically. Almost half IVF female patients report that infertility was the most troubling experience of their lives. Most IVF patients report symptoms of depression, anxiety, anger and isolation after an unsuccessful treatment and many of these symptoms exist for long periods of time. According to a retrospective study - 86 couples who have not succeeded in conceiving after IVF treatments, it was found that $66 \%$ of the women and $40 \%$ of the men reported on symptoms of depression due to failure of their IVF treatment and about one third reported on symptoms of depression 18 months later. The main reason due to which patients stop a treatment is a psychological burden of an IVF process rather than the financial burden involved in it as was a common thought in the past. Additional reason for stopping a treatment - lack of success (Domar, 2015).

The chances of conceiving are about $72 \%$ should infertile spouses be willing undergo recurrent fertility treatments. However, the part of patients that do not withstand the recommended 
treatment is around $15 \%$ for insemination and $22 \%$ for IVF. Factors that increase vulnerability of patients to lack of adaptation psychologically, during treatment, might affect the willingness of patients to withstand the treatment demands (Lopes et al., 2013).

For fertility patients who undergo treatments, a history of depression constitutes an extremely strong risk factor for future depression. Men and women with depression prior to fertility treatment, have probably less physical, emotional and social resources in order to cope with the pressure of fertility treatments and failure of a treatment and therefore might be considered as a "risk group" (Brennan et al., 2014).

\subsection{Effect of fertility treatment upon intimacy}

Most fertility patients undergo the treatment as a couple, so in addition to the challenge that fertility treatments face them with, they substantially influence their intimate relations and put them to a test. Endurance of long hard tasks involved with suffering and uncertainty might strengthen or destroy intimacy. Spouses experience difficult, complex and wearing experience, they both are in a state of emotional need and therefore find it difficult to be attentive to each other and support each other and it is often possible to see reactions of interiorization and anger towards a spouse.

Spouses might pay the price of treatments by a decline of their intimacy and damage to their sex life, and the reasons can be starting with thoughts about the longed-for baby in intimate moments, or due to a presence of a third party or any medical intervention that might turn sex life from an experience of love and closeness to a means of conceiving as sex becomes less spontaneous and performed only for reproduction purposes and limited to the period of fertile days. This fact can drain the mental energy and even sabotage sexual attraction existing between the spouses. In first stages of treatment, most couples exhibit coping capability, however, this capability decreases after about two years and many find themselves in a situation of harm to intimacy in general and to sex life in particular (mostly a rise in intimate conflicts and adjustment difficulties are perceived) (Ginor, 2015).

Lack of sexual functioning amongst couples in fertility treatments is commonplace, it was found that sexual and personal life quality amongst spouses is low and that is with or without diagnosis of infertility of a male source. Furthermore, the male spouses amongst infertile couples reported on less sexual satisfaction than their female spouses, possibly due to a psychological pressure involved in efforts to conceive or the effort to time the sexual intercourse around ovulation period of the woman - which causes a rise in stress level. Infertile men might experience 
feelings of incompetence, anxiety, guilt and depression. Those negative feelings contribute to the distress and are related to problems in male sexual functioning (Song et al., 2016).

Ferreira et al., indicated in their paper from 2014 that there are two opposite viewpoints on the subject of satisfaction in marriage, on one hand, fertility is perceived as an experience supplying opportunity to spouses to grow by it operating and serving as a challenge increasing the unity between spouses and creating new communication lines and solving problems, on the other hand, infertility constitutes a turning point from which various characteristics of married life can be emptied due to an awakening of issues spouses have not come across until that time.

Faria et al. (2012) indicated that in most cases, infertility has great impact on emotional condition of the spouses, which creates emotional disturbances of an individual or on the intimacy and might contribute to development and/or worsening of problems in married life.

\subsubsection{Emotional distress amongst women}

Infertility has social stigma which has destructive impact on health of a woman. In many specific cultures, there is great pressure on women to bring a child to the world and occasionally she is the one being cast out and blamed for not delivering am offspring and an inability to deliver a child even constitutes reason for divorce. Social implications of infertility mostly affect women and some women agree to undergo the treatments just to preserve marriage (Hagela et al., 2016).

According to an article by El Kissi from 2013, incidence of mental distress amongst women with infertility is between $48 \%-96 \%$.

Several studies were conducted on the subject of whether (or not) anxiety or depression contribute to infertility as a primary factor. A European study revealed that it takes more time for anxious women to conceive and they tend more to miscarriages than women with lower levels of anxiety (Hagela et al., 2016).

Infertility and pressure of medical treatment might put women at a risk of depression symptoms especially after failure of a treatment. On the other hand, women with depression symptoms might have higher chance of experiencing infertility due to impact of depression on biological mechanisms influencing manufacturing of hormones and ovulation (Brennan et al., 2014).

Fertility treatments are often involved with intrusive examinations and proceedings for a long period of time, administration of intricate medical drugs according a predetermined program - 
all these add to the pressure of diagnosis. Psychological influence of hormones women intake during fertility treatments were have not been properly researched, even though fluctuations in mood and depression are known as side effects of ovulation induction medical drugs and that women undergoing ovulation induction often complain about psychological problems.

It is common knowledge for a long time that depression is twice as common in women then in men and that women are at a higher risk of developing depression during their fertility years. The primary cause of mood problem a woman experiences during fertility treatments is unclear, as it is known that there is a connection between mood symptoms and hormonal fluctuations. Probably part of the distress women experience during ovulation induction/insemination or a cycle of embryo transfer is connected to the hormones they receive. In a study done recently, symptoms of mood and anxiety were examined in women undergoing IVF treatments / embryo transfer, a moderate rise in depression and anxiety indexes was observed during entire treatment cycle.

The highest depression levels occurred as response to decrease of estrogen levels. The role of progesterone was examined as well, usually, progesterone is considered as a contributing to depression and in that study, higher levels of progesterone are correlated with lower levels of depression. There are multiple causes for these symptoms, including psychological problems involved with a need to undergo fertility treatments, duration of infertility affects as well as to how a woman can be susceptible to symptoms of depression and anxiety. Currently, there are evidences for the fact that amongst women who have received hormonal treatment in fertility treatments, changing levels of hormones, especially low levels of estrogen will affect mood in addition to a psychological distress (Lake, 2012).

In a study conducted by Domar et al. (2012), 40\% of barren women felt "embarrassed" and more than half, 55\%, agreed that they feel "not woman enough" or "damaged as women" $(58 \%)$, and that they have waited too long to conceive (58\%). Women who have been treated, tended to feel more hurt and emotionally overwhelmed and have significantly focused on trying and coping than those who have not been treated. The most reported negative emotions for all women were those of frustration and impatience. Women who have been treated, were more exposed to feel "vulnerable" and "emptiness" while those who have not been treated felt more "confused". When one considers treatment that involves injections, women had mixed feelings while most sensed "hope" 46\%, and after it, "anxiety" $39 \%$. 
Even though a big obstacle to treatment being emotional, women have as well concerns regarding functional problems such as the injection process, the possibility of multi-fetal pregnancy, timing, consequences of cost of treatment and the side-effects. Fear of failure was the most significant emotional barrier to treatment, while most (72\%), quote a sense of anger when treatments "do not work" as a main problem.

One of the renown side-effects at time of fertility treatment is gaining weight, which hurts a woman's body image. In a paper by Ofra Lior (supporter, guide and coach of couples in fertility treatments) from 2009, many a woman tends to assign the blame for their gaining weight upon the hormones injected into their body and family members and the environment understand it. However, in fact, hormones do not cause directly gaining of weight, some of the hormones affect the stress hormones that might stimulate secretion of insulin and create a feeling of hunger; however, this is an indirect effect that is possible to overcome.

The reasons for gaining weight at time of fertility treatments is slightly similar to other cases: consumption of excessive calories the cause of which is not in medications, but rather in the vast stress a woman is in during treatments, increase of time spent at home, self-renunciation and reduction in physical exercise due to "over-prohibitions" a woman imposes upon herself to assist in success of treatments. Most smoking women stop smoking before beginning of treatment and food being a sort of substitute in the curse of stop of smoking.

Opposite to her, Orna Malki Livne who deals in the field of nutrition for pregnant women, claims that although gain of weight during fertility treatment is a result of excessive consumption of calories, however, hormones especially in amount injected, disrupt operational mechanisms in the body in more intense levels than in periods of pregnancy and menstruation, and it is common knowledge that excessive hormonal operation is expressed, amongst other things, in accumulation of fluids and a rise in appetite and therefore, in an operational body in which hunger and satiety system is disrupted due to hormonal activity with combination of environmental and emotional factors, there is a chance it will bring about gaining of weight even should a patient insist on balanced nutrition and physical exercise.

An article by Haelyon from 2010, indicates that IVF treatments manufacture a complex and unique experience for women which includes at least 3 dimensions: body, identity, and soul. The uniqueness of the feminine physical experience is hidden in the woman and she becomes the subject of medical treatment even when a fertility problem stems from the male spouse or when its cause is unknown. Regardless of source of a problem, a woman's body would always 
undergo hormones injections, invasive interventions, weekly Ultrasound examinations, egg retrieval and other procedures including hospitalization and anesthetization.

An additional experience regarding women is "identity confusion". In Israeli context, "feminine identity" relates to conceiving, birth and motherhood. While fertility treatments realize the yearning for motherhood, they as well emphasize an inability of a woman to become a mother. Research literature regarding this impact indicates that the feeling of "damaged identity" following fertility problems causes stress, social isolation, frustration and conflict with the environment. Women who suffer from infertility probably tend to alienate from social events with presence of little children.

Alongside with experiences related to body and identity, IVF female patients encounter as well emotional challenges, such as dealing with physical pain caused by surgical intervention. Furthermore, IVF treatments are involved with appearance of a new series of feelings: feeling of discomfort towards therapists, sorrow and despair after a failed treatment cycle, anxiety regarding maintaining intimacy and more. Women tend to consider themselves as responsible for the fertility problem and they do not involve the male spouse in this responsibility.

\subsubsection{Emotional distress amongst men}

Like women, men suffer from coping with fertility treatments both physically and psychologically. While there are many evidences of emotional adjustment of women to infertility, there are no systematic studies focusing on psychological adjustment of men to infertility and the treatments accompanying it and most of the studies regarding psychological aspects of infertility focused only on effect of infertility amongst women (Mikkelson et al., 2012).

In a study by Martins et al. (2016) it appears that symptoms of lack of adjustment amongst men have increased significantly about one year after the primary fertility assessment.

Evidences of anxiety, depression, sense of disaster and difficulties in communication with the female spouse, were found.

Man's infertility influences his life and might cause a life crisis. Although fertility treatments have become common in recent years, men often feel alienated and detached from a treatment process. Fertility treatments relate to gender asymmetry; therefore, a woman is the patient and most part of the treatment is aimed at the female spouse. The role of a man is mostly reduced 
to giving sperm sample in time, this applies as well on couples in which the problem is with the man.

Reports in the media usually represent infertile men as vulnerable and insinuate that male infertility threatens the perception of manhood as well as sexual performance. Men with a fertility problem were more distressed than men with other problems (Mikkelson et al., 2012).

In several very traditional cultures, infertility is perceived only as a woman's problem and therefore, a diagnosis of male cause is not acceptable. The negative impact of a diagnosis of "male infertility" on sexuality of a man was clearly proved in number of studies, for example, one out of every nine subjects was unable to supply the required sperm sample after he was informed about a deficient sperm quality in previous examination. Additional example: when a man who is supposed to supply a sample for IUI, he finds it difficult due to pressure imposed upon him following it. And eventually, the effect of fertility treatments - from timed sexual intercourse to surgical interventions is in many cases very extremely towards men. In a study conducted in a fertility clinic $-45.4 \%$ out of 487 men reported that having sex according to the clock is the most stressful, in $26 \%$ of men erection problems appeared after unsuccessful TESE as compared with $0.4 \%$ of men undergoing a successful TESE (Wischman, 2013).

Additionally, in an article of Wischman (2013) - until recent years, women were at the center of attention of fertility treatments and the responsibility for fertility deficiency was assigned to them as well and a man was required to be accepting and supporting. Currently, it is known that men and women have similar responsibility for infertility and therefore, greater attention must be given to mental experience of a male patient and his place not only as a supportive spouse, but as a partner being responsible for infertility. In most public as well as private fertility clinics, there is no requirement for participation of mental health personnel in a treatment or as external assistance and there are as well still no protocols regulating method of corporation between a mental health professional and the medical team and the role of medical personnel in initial mental support for fertility patients.

\subsubsection{Differences between men and women}

Wiscman (2013) mentioned that for $49 \%$ of women however only $15 \%$ of men, infertility is considered as the most upsetting experience of their lives. In a study he conducted regarding the psychological impact of infertility on women and men, it was found that there still are differences between genders: women are more negatively emotional than men. 
According to a paper by El-Kissi (2013), in an Italian study, 14.7\% of women had anxiety symptoms and $17.9 \%$ had symptoms of depression as compared with only $4.5 \%$ of men who had anxiety symptoms and 6.9\% who had depression symptoms. Furthermore, an American study conducted in 2011, which compared indexes of psychological distress between men and women facing an IVF treatment, found that the scores of psychological distress were higher amongst women than amongst men for symptoms of depression, state of anxiety, specific fertility problems and general stress.

After receiving results of a pregnancy test after IVF/ICSI, one out of four women and one out of 10 men have depression disorder and one out of seven women and one out of 20 men suffer from anxiety disorders (Gameiro, 2015).

Women are more susceptible to anxiety, stress, depression, and/or psychiatric illnesses than men. Patients with prior history of vulnerability of mental health disorders are more susceptible to experience depression, anxiety and/or psychiatric illness in a course of treatment (Zaig et al., 2013).

Many a study attempted to explain the psychopathological mechanisms of psychological distress in infertile couples, they assumed that coping and attachment strategies are different according to gender, as there is difference in infertility perception between women and men. Women perceive their infertility as a threat and loss more than men, which intensifies their distress (El-Kissi, 2013).

As compared to infertile, men, barren women express higher distress regarding infertility and tend to initiate more in a pursuit after treatment possibilities. These data indicate that women are more willing to undergo the required treatment for achievement of parenthood (Lopes et al., 2013).

The motives for searching for help differ between men and women, women find that support is efficient as it explains their reaction and legitimizes them while men seek information and practical advices (El-Kissi, 2013).

As indicate by O'Donnell (2009) - psychological support is still being offered, especially for women. Men in particular, indicate that they can overcome their feelings on their own and therefore, men are usually not defined as a target group for psychological consulting. 


\section{Coping with fertility treatments of women}

\subsection{Definition of coping}

Over last 20 years, an interest exists in how people handle/cope with stress - which is the coping process. The most common definition for coping is by Lazarus and his colleague Susan Folkman who defined coping as cognitive and behavioral efforts changing incessantly in order to manage specific external and/or internal demands which are perceived as incumbering burdensome one's resources and thus jeopardizing his health or well-being. Lazarus and Folkman emphasize the relationship between a person and his personal character and the events occurring within the environment, out of a recognition that the extent to which events are defined as pressure is dependent upon cognition of a person regarding these events and how he evaluates them with regard to his abilities to cope with the situation. Pressure occurs when competing demands lead to overload, inability is perceived as either preventing an event or coping demands of a situation and therefore, certain measure of pressure would be created (Bilney \& Pillay, 2015).

The definition relates to cognitive, emotional, and behavioral aspects of the coping process and focuses as well on the effort related to response of an individual, as well as conscious or unconscious behavior directed at managing a stressful event. Lazarus and Folkman (1984) indicated as well that stress management includes: reconciliation/acceptance, tolerance, avoiding or narrowing stress factors as well as according to a more traditional viewpoint of coping, a sort of control over the environment. Furthermore, coping is not limited to successful efforts, but rather includes any intentional attempts to control the pressure regardless of their efficiency. Coping is a dynamic process that changes over time while responding to demands according to objective and subjective evaluations (Frydenberg, 2014).

Stressors are events or situations evaluated as being discouraging which stimulate stress response that burdens the physiological and psychological resources of a person, as well as stimulating a subjective situation of physical or mental stress. Regardless of extent of their severity, stressors might cause physiological and behavioral disturbances beginning with psychiatric disturbances to a malfunction in the immune system (Anisman \& Merali, 2009).

As indicated by Folkman (2010), “coping is a critical point of embarking upon defending mental and physical health from damaging effects of stress". 
Stress is not always inherently bad, as a matter of fact, moderate levels of stress which Lazarus termed eustress (defined as positive response to stress factors) can in fact improve performances and provide a sense of achievement or accomplishment. Eustress is desired as a means for a healthier problem resolution. One requires certain amount of stress in order to retain survival, otherwise, nothing difficult would have ever been achieved and the human race would not have been able to achieve most of the progress that has occurred over time, therefore, pressure is essential for human development and assists adaptation (Bilney \& Pillay, 2015).

Gruner (2006) defines pressure as "mental or physical state of pain, sorrow, suffering, misery or inconvenience". High level of stress is not supposed to last long as it causes distress, however, lack of stress occurring over a long period of time can as well cause distress.

When low stress or low arousal low performance is manufactured and when stress is high, there is as well decrease in performance. There is a level in which stress is moderate and then positive pressure - eustress occurs, in this stage, performance is optimal. This model has drawn criticisms due to the fact that it is considered as too simplified by nature and it does not distinguish between different types of pressure which makes the related stress situation both a challenge and an obstacle (Yarkes \& Dodson, 2007).

\subsection{The Transactional model of stress and coping}

The Transactional model of stress and coping constitutes a frame for appraisal of processes of coping with stressful events and adaptation to a stressful experience is determined according to the interaction of changing cognitions of situations and between choosing of efficient coping strategies (Gourounti et al., 2014).

Interpretation of Lazarus and Folkman (1984) of stress focuses on transaction between people and their external environment. According to the model, stress cannot be stressing factor should a person not consider stress as a threat, but rather as being positive or even challenging. Additionally, should a person have proper coping skills and be able to use them, stress not necessarily can be a result of or develop due to the stressing factor. The model proposes to people to manage their stress and cope with it, they might learn how to change their viewpoint regarding the stressing factor and provide them with an ability and confidence to improve their lives and cope with any type of pressure as shown in figure 1. 


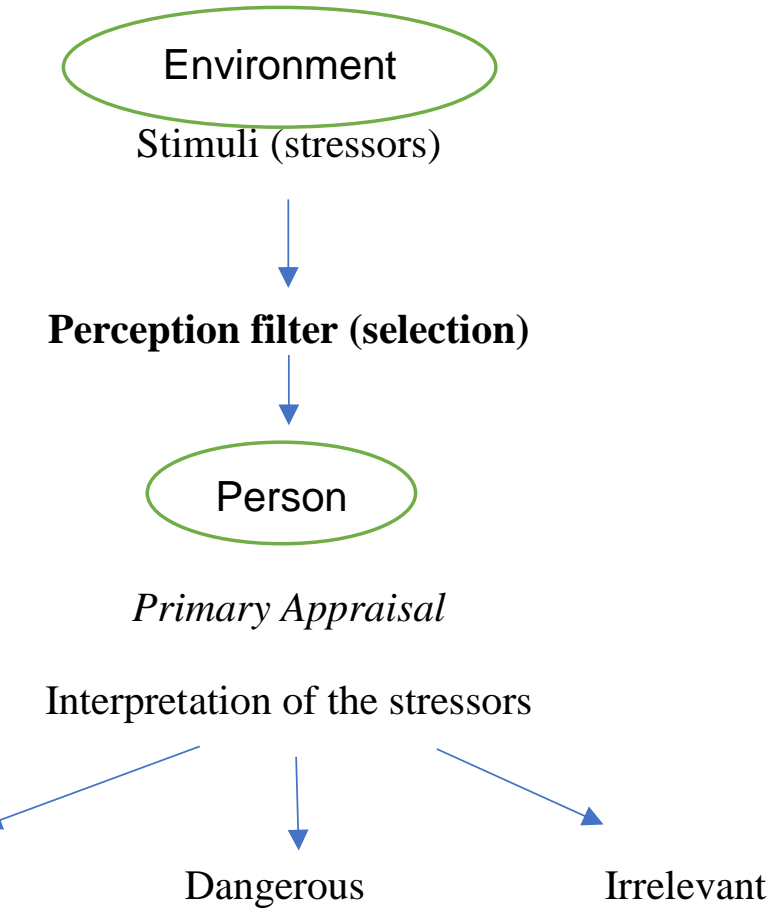

Positive

Challenge, threat harm-loss

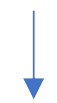

Secondary appraisal

Analysis of available resources

Insufficient Resources

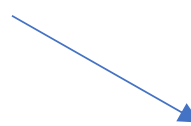

Problem-focused

Change situation itself
Sufficient Recourses

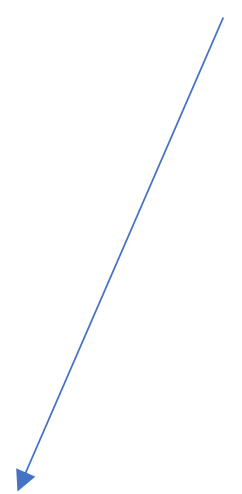

Overcoming stress
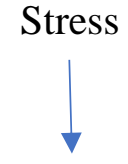

Coping
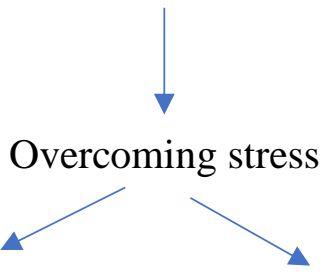

\section{Emotion-focused}

Change relation of the situation

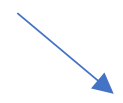

Reappraisal 


\section{Pacing and learning}

Figure 1: Transactional model of stress and coping of Richard Lazarus

To summarize, a number of factors can dictate a person's response to environmental stress factors, including characteristics of stress factors (for example, type of a stress factor and its control ability, capability of prediction and its chronicity, biological factors (meaning age and genetics) and previous history with stress factors and past experience (Anisman \& Merali, 2009).

\subsection{Coping strategies}

Lazarus and Folkman (1984) proposed two types of primary ways of coping:

Emotion-focused coping - involved to reduce negative emotional responses connected with stress, such as: embarrassment, fear, anxiety, depression, excitement, and frustration. This option can be efficient when stress origin is exterior to one's control. Medication treatment can be given as emotional coping as it focuses on the stimulating factor of stress rather than the problem. Other emotion-focused copings include:

- Diversion, such as be busy with something to not deal with the problem.

- Emotional exposure - involved in expressing a strong feeling by talking or writing about negative events which brought about those feelings.

- Alienation, self-control, self-accusation.

- Excessive eating - "comfort" food.

- Consuming alcohol.

- Suppression of negative thoughts or feelings. Suppressing feelings for a long period of time hurts immunological capability and leads to low physical health.

A meta-analysis conducted by Peneley and Weibe (2012) revealed that emotion-focused strategies such as: eating, drinking and medications reported on reduced health results, these strategies are not efficient when ignoring the basic reason for stress. 
Emotion focusing does not provide long-term solution and might have negative side-effects as it hinders one from coping with the problem. However, it can be a good option when stress is external to one's control. Differences between sexes were reported: women tend to use emotion focused strategies more than men (McLeod, 2014).

Problem-focused coping - in problem resolution, stress factors are being directed in certain ways which cope with a problem or a stressful situation which causes stress and as a result directly reduce the pressure. Problem-focused strategies that are aimed at reducing the stress factor include:

- Problem resolution

- Managing or changing the situation and search for information

- Accepting useful social support

- Planning and direct action

In general, problem-focused coping is the best, as it removes the pressure, deals with the root of a problem, and supplies a long-term solution. However, it is not always possible to employ problem-focused strategy, for example in a case when sense of loss accompanies fertility patients, those strategies will not be particularly useful, as coping with the sense of loss requires emotion-focused coping. Moreover, problem-focused approach will not work in any situation where remove the stress is beyond one's control. They operate better when one can control the source of stress.

This method is not productive for all people, meaning, not all people can control the situation or see a certain situation as solvable, for example, optimistic people with positive expectations from the future would probably use problem focused strategies as opposed to pessimistic people who tend more to use emotion-focused strategies (McLeod, 2014).

Following these two approaches, Carver et al. (1989) proposed a third dimension, meaning avoidance coping - which is of importance as well and includes approaches considered in the past as being emotion-focused, such as cognitive actions and changes intended to avoid a stressful situation or to diminish thoughts and emotions connected with it. In this coping style, efforts are directed at reduction, denial or ignoring the occupation with a stressful situation and even though some researchers added avoidance coping with emotion-focused coping, the 
styles are conceptually different. Avoidance coping is focused on ignoring a stress factor and therefore, it is passive while emotion-focused coping is active (Karekla, 2010).

Avoidance coping was found to be connected with a bigger distress than other coping styles. In general, people who employ this coping style experience higher dis-functioning.

Avoidance coping is positively correlated with depression symptoms. In a ten-year longitudinal study, a positive correlation was observed between avoidance coping and between stress and additionally, anxiety and depression might occur as avoidance coping fails to remove minor stresses and hence, the stress factors become bigger and more stressful, and as a result, one experiences increased anxiety and depression. A negative circle can develop when depressed people might appraise their ability to cope with stress factors when these are low and to be more pessimistic regarding future results. This negative thinking might lead them to cope by more passive coping styles, such as avoidance coping and thus, the negative circle continues (Holanan et al., 2005).

Dyadic coping - achieved as a process of tension communication that initiates the coping response of both spouses. This dyadic reaction is activated when one of the spouses who appraises the stress communicates with their spouse and later the spouse perceives and interprets the exuded stress signal and responds through positive or negative coping strategies in an attempt to diminish the sense of stress of the spouse (Levesque et al., 2014).

Bondenman (2008) described several different dyadic strategies in the context of spouse relationship:

- Negative dyadic coping - relates to a display of lack of interest or exhibiting sarcasm towards the stress of a spouse. Employing coping strategies which are not authentic and in response to the involuntary exuded stress signal or not to believe that the spouse is of need of support.

- Noble dyadic coping - relates to when one spouse adopts additional tasks in order to relieve the burden of another.

- Common dyadic coping - relates to both spouses operating together in order to resolve the stressful situation together.

Dyadic coping serves two main roles: reducing the pressure of both spouses and improving the quality of relations. In situations when one spouse copes with a significant stress or when 
both spouses are challenged with same stress factor, dyadic coping strategy is supposed to assist in managing the distress. In addition to the purpose of reducing stress, dyadic coping is considered as cultivating a sense of welfare amongst couples, it is considered as vital to quality of relations as well, as dyadic coping cultivates mutual trust, respect, commitment in relations and a sensation that the relation is comforting and supporting. Empathic caring amongst women and assuming a perspective amongst men proved that these improve the dyadic coping strategies of spouses. Following those findings, it is possible to state that men are usually more comfortable when matter are being managed from a rational, logical and emotion-absent viewpoint, while women choose to focus on problems by their emotional response.

And therefore, it is possible that when people behave in a way that suits expectations of their spouses (meaning, when men play the cognitive component role and women play the emotional component role), a spouse will be more confident with their ability to cope with a stressful situation. The dyadic functioning of an individual is strongly correlated to their emotions, thoughts and behaviors (in this case, empathy and their dyadic coping), particularly when these are opposed to their relation factors (Levesque et al., 2014).

In summary, the initial ability to share an emotional response being felt by posing yourself in another's footsteps, operates directly on dyadic coping strategy and on behaviors (Levesque et al., 2014).

Literature distinguishes between coping as a stable personal trait and a dynamic one that changes according to state (Lazarus \& Folkman, 1984). When such a big differentiation exists in type of events, their intensity and time of occurrence, it seems that the choice of certain strategy would be influenced by complex of components that compose the situation, the environment, the person and interaction between them (Lazarus, 1993).

A certain strategy might not be efficient under one group of terms, however, be efficient under other terms. Eventually, the ability to maintain flexibility and be prepared to use various strategies could be the hallmark efficient coping (Anisman \& Merali, 2009).

\subsubsection{Coping with infertility and fertility treatments}

Couples can report on a feeling of threat to the relationship occurring simultaneously. As infertility can be quite a stressing experience, couples usually make various behavioral or emotional efforts to manage, change or regain control over their lives. These coping strategies 
are methods by which men and women can cope with their infertility problems even though they do it by different methods. Couples who take active avoidance coping look for a relief of pressure by active avoidance of situations and of reminders of their fertility problems. The findings that women's use of active avoidance coping reduces marital benefit of relations with their male spouses supports previous studies testifying to the fact that active avoidance coping brings about increased stress amongst male spouses. However, there was an unpredictable finding that a man's use of this strategy increased marital benefit of his female spouse.

The gender difference might allegedly be the extent of emotional support each of the spouses can offer to the other. Considering that men sense this burden of caring for their wives while they undergo treatments, use of avoidance coping might be perceived by a woman somehow as joining her suffering and thus create a perception of increased understanding and support. Couples who use meaning-based strategies perceive positively the fertility problem, define the goals of life and values besides having children and believe that there is a meaning to their difficulties in giving birth to children (Peterson et al., 2011).

Gourounti et al. (2010) evaluated the effect of psychological variables on stress connected with infertility. Most of the studies included, examined the correlation between coping and emotional response to infertility and failed fertility treatment and found consistent negative influence of escape/avoidance, coping of self-accusation, coping with acceptance of responsibility, coping with positive reevaluation and coping of search for social support.

It was found that problem-managing coping strategies were correlated to higher levels of infertility distresses and worse adaptations, while emotional approach and strategies of problems appraisal (positive reinterpretation) were correlated to lower levels of infertility distresses and better adaptation (Benyamini et al., 2009).

A study conducted recently, has found that coping strategies of avoidance and active coping were positively correlated to higher levels of infertility distress (Lykeridou et al., 2011).

Ability of control infertility and fertility treatment is not related to psychological distress and it seems that there are differences between previous findings and that existing evidences are not yet conclusive. According to the researchers, only one study examined the correlation between perception of control capability and coping strategies and found that a sensation of loss of control over the lives of people due to infertility and fertility treatments was positively correlated to escape coping strategy and low perception of control capability is positively correlated to avoidance coping. Findings of the study revealed that high perception of control 
capability over infertility is positively correlated to problem-focused coping. In particular, high perception of treatment control capability was positively correlated to problem-managing coping. Therefore, it is possible to conclude that despite the fact that infertility is a condition of low control, perception of women regarding the treatment efficiency (treatment control capability) might cause adaptation of problem-focusing (Mahajan et al., 2009).

Women with high levels of stress related to fertility and a state of anxiety had lower perception of personal control than women with lower levels of stress and anxiety. Women with higher levels of stress related to fertility, state of anxiety and depression symptoms, have employed more often avoidance and emotion-focused coping strategies and often problemcoping (Gouruonti et al., 2010).

In response to stressful situations of low control, problem-focused coping strategies such as problem-managing coping, might have harmful influences as those motivations awaken elicit a sense of frustration and disappointment (Terry \& Hynes, 1998).

\subsubsection{Coping strategies after IVF failure}

In IVF / fertility treatments, as the time goes by without pregnancy, the situation turns gradually to a type of loss and therefore, a series of responses to sorrow occurs in a path towards acceptance and resolution of loss or a coping process. Usually, it is possible to conclude that problem-focused coping strategy leads to better adaptation whereas emotionfocused strategies are related to outcomes of lack of adaptation. However, it is possible that emotion-focused strategies are useful for coping with short-term events which are incontrollable. Coping strategies are conceptually complex and are differently classified in many a study. Therefore, both problem-focused coping and emotion-focused coping are not able to clearly demonstrate the real meaning of coping (Lee et al., 2014).

Terry \& Hynes divided coping strategies of couples who have experienced failure in IVF treatment as following: coping with appraisal problems (meaning, management of the appraisal of stress of a situation) is related to better adaptation whereas escapist strategies and problem-management (meaning, managing solution to a problem) are related to maladaptation.

It was found that after IVF treatment failure, women tend more to use the following coping strategies: confrontation (meaning, women wanted to find out more details about the problem and then to learn something new in order to cope with the problem), optimism (meaning, 
women tried to think positively and hoped the situation would improve) and relying on oneself (meaning, women kept their emotions to themselves and wanted to be alone in order to think things through) as efficient coping strategies, whereas emotional, palliative and evasive coping strategies were less efficient. Therefore, participants tend more to use problem-focused strategies than emotion-focused strategies to cope with psychosocial distress. Problem-focused strategies try to cope with the stressful situation itself while emotion-focused strategies try to cope with the variety of emotions awakened as a result of the situation (Lee et al., 2014).

As infertility is a stressful factor with low control, women can do extraordinarily little and even nothing in order to actively change the nature of the situation. As a result, passive coping styles and emotion-focused strategies which include efforts to focus on something else than the stress source and relieve the anxiety can also be suitable. While coping strategies themselves can influence directly the stress related to infertility, they can as well be used as mediator variables between social support and stress (Martins et al., 2011).

\subsection{Support systems}

The emotional impact of infertility creates problems not only to spouses, but rather to their family members and friends as well, and therefore social support can be a critical component of the way a woman adapts to the unexpected stress of infertility, especially as most women expose their infertility to others, and to a larger extent than men. Social support is defined as a perception that someone has an available confidant or that he experiences a caring attitude demonstrated by a specific source that is usually desired and given by the spouses, family, and friends. Social support is related to lower levels of depression and anxiety and reduction of stress of infertility (Martins et al., 2014).

According to Transactional stress theory, social support from a given context amongst other things can affect a cognitive appraisal of a stressful encounter together with coping strategies creates stress response.

Many people want to be useful and supportive, however they do not know how. Despite their good intentions and their efforts, they usually say and do the wrong thing, whether hurting a person or hurting themselves. An increasing number of parents, siblings, coworkers and employers come across similar harsh situations and they do not know how to respond. 
One of the main reasons for family and friends to find it difficult to help is that they know so little about the emotional aspects of infertility, starting with the shocking diagnosis and the demanding treatment to disrupting daily experiences. This emotional overload can cause one to become depressed, angry and filled with guilt, and family and friends often add to the pain with good intentions nondeliberate remarks and actions. Furthermore, people and mainly spouses, might cause damage should they not be confident regarding their emotions, not be able to handle pain of another person and changing moods, uncomfortableness with the sexual contexts of infertility or unwillingness to accept a coping method. Therefore, many people and couples often turn to support groups in which they can express the harm, the anger and the resentment that they cannot or will not expose in front of those close to them. They feel safer amongst others who share same pain (Mahlstedt, 2009).

People in fertility treatments might turn their anger and resentment towards their friends and loved ones and thus they feel more isolated and start avoiding social and familial gatherings, in which they feel unusual, embarrassed, or "unwanted" and thus a magic circle is created (Mahlstedt, 2009).

Appreciated familial support can influence the way a woman experiences stress of infertility both directly and indirectly. Findings of the study insinuate that perceived support from a family might assume a more direct role in relieving the stress of social infertility. Positive perceptions regarding the support a family provides not only reduce social problems related to infertility, but as well are indirectly useful in four fields of infertility stress (worrying regarding relations, sexual worry, rejection due to lifestyle without children and need for parenthood).

High level of support from friends can lower the levels of social stress of women by use of active coping as a coping strategy (Martins et al., 2014).

Relations with family and friends can be tense should the couple believes that they are not taken seriously, or should their decisions not be respected. This can as well occur amongst spouses. Additional problem arises when people need and expect a certain type of support that others do not know how to provide. Occasionally, people lose patience regarding an observed obsession of infertility. They have no idea how infertility influences each aspect of a person's life (Mahlstedt, 2009).

There is a strong correlation between support of a male spouse and stress in relations. This indicates that the coping strategy a woman adopts during achieving pregnancy through 
treatment has no bearing, the perception is that the moment the male spouse is supportive it can affect not only communication between the spouses regarding effect of infertility upon their relations but rather as well, for example upon their timed sexual relations (Martins et al., 2014).

A problem between the spouses might start when they are both hurt, stressed and depleted of physical and mental energy, less capable of fulfilling each other's needs and they feel apart. This is mainly true should their ways of coping be different like when one of the spouses who prefers privacy feels embarrassed or abandoned while the other is outspoken and has the will to talk and share with others (Mahlstedt, 2009).

Studies emphasize the positive effect of support on the part of family members and friends in psychological situations of couples with infertility, however, social attitudes towards births can cause couples to sense a social pressure to have children. Therefore, the question arises: too involved or not involved enough? Despite the fact that many a couple wants and appreciates support from family members and friends, couples feel pressure from their social networks and reported that "distance helps": to be apart from their families protects couples from questions of good intensions however incessant about infertility and the accompanied treatment. Many couples sense that people without fertility problem do not understand their situation and therefore are not being comforted by the proposed support in social networks. In general, many couples are socially isolated, however this isolation is occasionally enforced and there are couples who want support specifically from people with fertility problems.

To summarize: social support from family members and friends has positive and negative influences over stress levels of couple undergoing fertility treatments. Social and familial pressure for having children might add to the stress already existing upon the couples, therefore, support of family members, people who do not have fertility problem and friends cannot be perceived as useful (Read et al., 2013).

\subsection{Coping of single mothers by choice}

The fear of time window to close with it the possibility to childbirth to vanish motivates many a woman with a male spouse or alone, to turn to medical treatments that would help her bring a child into the world. The decision to bring a child into the world is not simple, about $85 \%$ of women arrive for treatments due to age and not as it being their natural choice (Naor, 2012). 
Most of single mothers by choice support the marriage institute and it was as well found that this is their first choice, however, their choosing single parenthood is related amongst other things to lack of ability in finding a suitable spouse alongside with the determination of these women to realize their motherhood (Segal \& Engelchin, 2008).

Additional motive to a decision to conceive is "social clock" and it is guided by social norms and sets a timetable according to which one is expected to follow their tasks of life and is even judged regarding their meeting this timetable. Family as cultural code as well dictates an individual their family life cycle (marriage duty, bringing legitimate children to the world and more). These codes might increase the indecision of women regarding giving birth to children in a late age when it is the last chance of realizing motherhood. The motive of choice of single motherhood should be profoundly considered - is the decision stems from pressure of environment, from age or from pressure of being alone and not necessarily from the real desire of being a mother (Nevo, 2015).

The Israeli society which sanctifies the subject of marriage and intimacy views bachelorhood as an unacceptable condition out of free choice of a person. Adult singles are perceived by society as less adults and less socially adaptive. Bachelorhood gains denunciation of merci and a variety of negative stereotypes and this is more obvious when the subject is single women. This difference is expressed in the multiple pressures exerted upon women and the stereotypes attributed to them and therefore, single women experience more social pressure regarding marriage and starting a family than single men (Shahar \& Sharon, 2010).

The predicted reaction of family members and friends can be divided to two: on one hand, many a single mother receives a lot of support and a lot of sympathy for their decision to have children, which is considered a central value. On the other hand, the decision might bring about offensive remarks, sometimes the family might exhibit disappointment towards parents who did not fulfil their dream and gave birth to children in the traditional way within a marital system or an orderly couple system. Additionally, there are parents who feel sorry for their daughter who is a single mother due to the great burden which she is expected to bear and opposite to them, others would admire her for managing to do everything alone.

Single mothers by choice with no dependency on the path they choose to conceive have extensive social networks composed of family, friends and additional single mothers by choice, while part of these networks was prepared by the women prior to their becoming 
mothers. These networks help these women mentally and financially and constitute a compensation for the lack of the intimate relation with the male spouse (Nevo, 2015).

\subsection{Coping of same-sex mothers}

One of the most significant decisions that same-sex couples have to make on their way to parenthood is which one of them would be the first one to conceive: is it right for the young one to conceive first or whether the older one is to be given precedence. This is not a simple decision and it might bring forth existing intimate charges and conflicts. Like in a heterosexual family in which a man senses as being outside the frame and competes with the baby, it might as well happen when a lesbian couple, therefore there is importance to discussion and to giving profound expression to feelings of the person who still has not gave birth. There are as well compromises to go through the procedure together, meaning to conceive at the same time. This can be an exciting experience that can tighten the intimacy between the spouses, however, mutual support in pregnancy can be not an easy coping, not only due to the fact that they would have to share moments of anxiety, unpleasant examinations, etc., while both are in a "sensitive" state.

Additional subject that should be considered is that pregnancy and the process of achieving it is going to be not natural. Despite the fact that both female spouses are fertile and healthy they would have to conceive medically, meaning through fertility treatment, which means at least one of the female spouses would have to undergo various tests, to cope with embarrassment of an invasive process of IUI and it is possible that in the future, a more complex stage of fertility treatments will arrive that would include injections and hormones and even IVF. If that is the case, and thus although the physical burden would fall on one of them, however, similarly to heterosexual couples, emotional, mental and intimate overload would be common to both. This can be a complex period that would serve as a test for intimacy, bring about the tightening of relations or even disintegrate them, maybe hurt intimacy (Glinor, 2012).

In addition to the anxiety accompanying a fertility treatment, lesbians as well suffer additionally from stress factors relating to their being a minority in fringes of society (for example - discrimination) (Holley et al., 2015).

Same-sex family faces the challenge of coping with the place of genetic charge in parenthood. In these families, biological-genetic relation exists only with one parent while the other parent is not a biological parent and defined as a guardian or adopting parent. Even should the 
spouses prefer to keep biological parenthood issue hidden and unsolved and mark it as irrelevant, reality does not allow it (like the right for maternity leave which is awarded only to a biological parent).

In fact, all parenting exceeding the acceptable pattern in society is in defense and must gather scientific evidence in order to receive the social approval it needs so much and to dispel doubts regarding inadequacy of the different for parenting task, as well due to the fact that in that intimacy there is no representation of both sexes (Aviram, 2011).

\section{Chapter 2- Aim and objectives}

The emotional aspects are integral to coping with the difficulties of infertility and treatments, and many studies have indeed focused on them with respect to couples. This study, however, also examines the coping of women who have received far less attention in the literature. This study therefore examined the difficulties and support systems of women in three different family frameworks: traditional family headed by a heterosexual married couple, single-mother families, and same-sex families.

Five research questions were designed to examine:

1. Does the family structure have an impact on a woman's ability to cope with fertility treatments?

2. Is the dyadic support system of a heterosexual couple necessarily more stable than that of the support systems of single women or lesbians?

3. Does the type of treatment influence women's coping with fertility treatments?

4. Do women with children cope differently than women without children?

5. Does a patient's age affect her coping with fertility treatments? 


\section{Research hypothesis:}

1. A patient's family structure affects her coping method with fertility treatments.

2. The dyadic support system for married women is more stable as compared to the support system of lesbians and singles.

3. IVF treatment has a positive correlation with the coping method.

4. Coping difficulty has a negative correlation should spouses already have children.

5. Woman's age has a positive correlation with motivation for coping with fertility treatments.

\section{Objectives:}

\section{General objective}

To examine whether there are differences in the way women from different family structures cope with fertility treatments.

\section{$\underline{\text { Specific objectives- to examine }}$}

1. the existence / absence of support systems during fertility treatments as well as kind of support.

2. The stability of the dyadic support of married women compared to that of the lesbians.

3. To examine whether there is a difference in the way of coping if the women have children or not

4. If younger women and older ones cope differently with treatments.

\section{Chapter 3: Methodology}

\section{Research design}

A mixed-method research design was used in this study and quantitative and qualitative data were collected simultaneously. Combining data made it possible to better understand and deepen the interpretations of the findings. Quantitative and qualitative methodology each has limitations and biases; the purpose of using both was to overcome the biases and thus contribute to the findings' validity (Johnson et al, 2007). 
The quantitative part is based on a survey research study which use of self-report measures on carefully selected samples. information is being collected from a sample of individuals through their responses to questions, using a questionnaire include a series of items reflecting the research aims. this method used for collection of information from a pool of respondentswomen undergoing fertility treatments as in this study, by asking multiple survey questions. survey researchers have a strong preference for large random samples because they provide the most accurate estimates of what is true in the population (Check \& Schutt, 2012)

The qualitative study based on the phenomenological approach which have emphasized the role of subjective experience, the goal of phenomenology is to describe the meaning of this experience both in terms of what was experienced and how it was experienced. It is also developing a complete description and understanding of a human experience or experiential moment (Neubauer et al, 2019).

The rational of selecting those methods:

-For the quantitative study: conducting this type of method to collect answers regarding definite, significant questions to report directly on the thoughts, feelings, behaviors, coping methods, support systems and difficulties of women undergoing fertility treatments. This research type includes recruitment of individuals, collection, and analysis of data (Ponto, 2015).

-For the qualitive study: to understand the meaning of women undergoing fertility treatments lived experience, a phenomenological approach was chosen in order to explore what women experiences, focuses on their experience of a phenomena and exploring it from the perspective of those who have experienced it. using this approach complete description of human and meaning (Beutler et al, 1998).

Participants included women with similar religious affinity as well as being treated in the HMO's institutions -where the ethics committee approved conducting the study, to standardize responses. in general, fertility treatments are conducted at HMO's women's healthcare clinics. In three main clinics such as: Tel-Aviv, Modiin and Rishon Lezion IVF treatments are also conducted in addition to the conventional treatments in collaboration with Assuta medical centers located in Tel-Aviv and Rishon Lezion. While the first stage of ovulation induction and the follow-up is performed at the HMO and the second stage where operating room and the lab work are required are performed at the two medical centers mentioned. 
Patients who came to the clinic for appointment or to perform tests and were found eligible to the study's criteria were asked to participate in the study and the questionnaire was given to them during their stay at the clinic. As for the qualitative part - recruiting five participants for the preliminary study (pilot study) was by convenience sampling- patients whom I'm familiar with were asked their permission to conduct the interviews at the clinic or at their homes, according to their preference. Saturation of data was reached after 15 interviews, when I recognized that I have heard themes before in multiple prior interviews, also Guest, Bunce and Johnson (2006) suggested that between six to twelve interviews with the target population is adequate to achieve theoretical saturation.

\section{$\underline{\text { Avoiding risk bias }}$}

-For the quantitative study- I used a large sample of 450 participants who were selected randomly, the questionnaire questions were written in accordance with the analysis that was designed.

-For the qualitive part - the interviews were conducted following the guidelines, open ended questions were used to get information, at first the interviewees were asked general questions and then continued to more specific ones. The questions were simple, and I avoided leading questions. Maintained naturality as not influence the participant's responses nor judging them.

\section{Research population and sample}

All women have undergone fertility treatments (treatments with medications) and IVF treatments including frozen embryo transfer.

Excluded from this study were women:

- who needed a sperm donation due to a severe fertility problem of their spouses.

- who had undergone cycles of ovulation follow-up that did not involve a medical procedure (without medications).

- older than 45 who underwent egg donation treatments.

- who were surrogates.

- receiving fertility preservation treatments (cryopreservation of their eggs).

Quantitative part -table 1 summarizes the demographic variables of the participants. 450 women participated in this study. Age of women ranged between 22 and 45, with an average 
of 36.41 (Standard Deviation = 5.18). Education distribution showed that $3.8 \%$ finished Highschool, $16.7 \%$ had a non-degree certificate, $49.7 \%$ had BA and $29.8 \%$ had MA or higher degree. Sample was divided into three groups $-33.3 \%$ were married, $33.3 \%$ were single and $33.3 \%$ had a female partner. Among 300 women in this study who had a romantic partner, age of partner ranged between 24 and 54, with an average of 35.89 (Standard Deviation = 5.65). Partners' education distributed with 8\% finished High-school, 24\% had a non-degree certificate, $52 \%$ had BA and $16 \%$ had MA or higher degree.

Years of being in relationship ranged between 1 year and 20 years, with an average of 5.77 $(\mathrm{SD}=3.80)$.

About $50 \%$ of women in sample had children while, average number of children was 1.55 $(\mathrm{SD}=0.79)$.

Qualitative part- table 2 summarizes the demographic variables of the participants. 15 interviews were conducted ( 5 of each family structure), age of women ranged between 28 and 44, with an average of 36. Education distribution showed that $6.6 \%$ finished High-school, $46.6 \%$ had a non-degree certificate, $33.3 \%$ had BA and $13.3 \%$ had MA or higher degree. Years of being in relationship ranged between 2 years and 9 years with an average of 4.9. $33.3 \%$ of women in sample had children while, average number of children was 1.8 .

Table 1- Quantitative part - descriptive statistics of the demographic variables

\begin{tabular}{|l|l|l|l|}
\hline Marital status & Married & Singles & Same sex \\
\hline Age group & 12 & 0 & 8 \\
$22-27$ & $8 \%$ & & $5.3 \%$ \\
\hline $28-33$ & 55 & 0 & 67 \\
& $36.6 \%$ & 49 & $44.6 \%$ \\
\hline $34-39$ & 59 & $32.66 \%$ & 52 \\
& $39.3 \%$ & 101 & $34.6 \%$ \\
\hline $40-45$ & 24 & & 23 \\
\hline
\end{tabular}




\begin{tabular}{|c|c|c|c|}
\hline & $16 \%$ & $67.33 \%$ & $15.3 \%$ \\
\hline Education & 9 & 3 & 5 \\
\hline high school & $6 \%$ & $2 \%$ & $3.3 \%$ \\
\hline $\begin{array}{l}\text { non-degree } \\
\text { certificate }\end{array}$ & $\begin{array}{l}23 \\
15.3 \%\end{array}$ & $\begin{array}{l}22 \\
14.6 \%\end{array}$ & $\begin{array}{l}31 \\
20.6 \%\end{array}$ \\
\hline Bachelor's Degree & $\begin{array}{l}55 \\
36.66 \%\end{array}$ & $\begin{array}{l}72 \\
48 \%\end{array}$ & $\begin{array}{l}96 \\
64 \%\end{array}$ \\
\hline $\begin{array}{l}\text { Master's Degree and } \\
\text { above }\end{array}$ & $\begin{array}{l}63 \\
42 \%\end{array}$ & $\begin{array}{l}53 \\
35.3 \%\end{array}$ & $\begin{array}{l}18 \\
12 \%\end{array}$ \\
\hline $\begin{array}{l}\text { Age of the spouse } \\
24-31\end{array}$ & $\begin{array}{l}31 \\
20.6 \%\end{array}$ & - & $\begin{array}{l}41 \\
27.3 \%\end{array}$ \\
\hline $32-39$ & $\begin{array}{l}75 \\
50 \%\end{array}$ & - & $\begin{array}{l}75 \\
50 \%\end{array}$ \\
\hline $40-46$ & $\begin{array}{l}37 \\
24.6 \%\end{array}$ & - & $\begin{array}{l}33 \\
22 \%\end{array}$ \\
\hline $47-54$ & $\begin{array}{l}7 \\
4.6 \%\end{array}$ & - & $\begin{array}{l}1 \\
0.6 \%\end{array}$ \\
\hline $\begin{array}{l}\text { Spouse's education- } \\
\text { high school }\end{array}$ & $\begin{array}{l}17 \\
11.3 \%\end{array}$ & - & $\begin{array}{l}7 \\
4.6 \%\end{array}$ \\
\hline $\begin{array}{l}\text { non-degree } \\
\text { certificate }\end{array}$ & $\begin{array}{l}34 \\
22.6 \%\end{array}$ & - & $\begin{array}{l}38 \\
25.3 \%\end{array}$ \\
\hline Bachelor's Degree & $\begin{array}{l}68 \\
45.3 \%\end{array}$ & - & $\begin{array}{l}88 \\
58.7 \%\end{array}$ \\
\hline $\begin{array}{l}\text { Master's Degree and } \\
\text { above }\end{array}$ & $\begin{array}{l}31 \\
20.7 \%\end{array}$ & - & $\begin{array}{l}17 \\
11.3 \%\end{array}$ \\
\hline
\end{tabular}




\begin{tabular}{|c|c|c|c|}
\hline $\begin{array}{l}\text { years of marriage / } \\
\text { shared living } \\
1-3\end{array}$ & $\begin{array}{l}34 \\
22.6 \%\end{array}$ & - & $\begin{array}{l}74 \\
49.3 \%\end{array}$ \\
\hline $4-6$ & $\begin{array}{l}45 \\
30 \%\end{array}$ & - & $\begin{array}{l}54 \\
36 \%\end{array}$ \\
\hline $7-10$ & $\begin{array}{l}43 \\
28.6 \%\end{array}$ & - & $\begin{array}{l}3 \\
2 \%\end{array}$ \\
\hline $11-13$ & $\begin{array}{l}12 \\
8 \%\end{array}$ & - & 0 \\
\hline 14 and above & $\begin{array}{l}16 \\
10.6 \%\end{array}$ & - & 0 \\
\hline $\begin{array}{l}\text { number of children } \\
0\end{array}$ & $\begin{array}{l}58 \\
38.6 \%\end{array}$ & $\begin{array}{l}99 \\
66 \%\end{array}$ & $\begin{array}{l}65 \\
43.3 \%\end{array}$ \\
\hline 1 & $\begin{array}{l}48 \\
32 \%\end{array}$ & $\begin{array}{l}44 \\
29.3 \%\end{array}$ & $\begin{array}{l}46 \\
30.6 \%\end{array}$ \\
\hline 2 & $\begin{array}{l}31 \\
20.6 \%\end{array}$ & $\begin{array}{l}7 \\
4.6 \%\end{array}$ & $\begin{array}{l}25 \\
16.6 \%\end{array}$ \\
\hline 3 and above & $\begin{array}{l}13 \\
8.6 \%\end{array}$ & 0 & $\begin{array}{l}14 \\
9.3 \%\end{array}$ \\
\hline
\end{tabular}

Table 2- Qualitative part-demographic data

\begin{tabular}{|l|l|l|l|}
\hline Marital status & Married & Singles & Same sex \\
\hline $\begin{array}{l}\text { Number of } \\
\text { participants }\end{array}$ & 5 & 5 & 5 \\
\hline Average age & 31.2 & 42.6 & 33.2 \\
\hline
\end{tabular}




\begin{tabular}{|l|l|l|l|}
\hline Education & - & 1 & - \\
\hline $\begin{array}{l}\text { non-degree } \\
\text { certificate }\end{array}$ & 2 & $20 \%$ & 4 \\
\hline Bachelor's Degree & 2 & 1 & $80 \%$ \\
\hline $\begin{array}{l}\text { Master's Degree } \\
\text { and above }\end{array}$ & 1 & $20 \%$ & 1 \\
\hline $\begin{array}{l}\text { Average years of } \\
\text { marriage / shared } \\
\text { living }\end{array}$ & 6.4 & $40 \%$ & $20 \%$ \\
\hline $\begin{array}{l}\text { Average number of } \\
\text { children }\end{array}$ & 1 & $20 \%$ & - \\
\hline
\end{tabular}

\section{Research tools}

The semi-open questionnaire used in this study was consisted of three parts:

1. demographic questionnaire, asking participants to indicate their age, level of education, their spouse's age and his level of education, number of years of marriage / shared living, and number of common children.

2. The fertility problem inventory (FPI; Newton et al., 1999), which takes a comprehensive approach to evaluating stress stemming from infertility, by measuring the effect upon dimensions of social life, sexual life, and intimacy and the importance of parenting in the lives of fertility patients. The FPI indexes contain 46 items, the scores for which are organized on five subscales and one global score composed of all previous factors. The subscales are social concern (10 items), for example: "To what extent do family / friend get-togethers affect you?"; sexual concern (8 items), for example: "How much stress has your fertility problem caused in your sex life?"; caring for the intimacy (10 items); the need for parenting (10 items); and rejecting lifestyle without children (8 items).

According to Newton et al., Cronbach's $\alpha$ for these five indexes ranges between 0.77 and 0.87, showing good reliability (while the general stress scale has Cronbach $\alpha$ of 0.93). The 
FPI was extensively studied by, among others, Van Der Broek et al (2010), Slade et al (2007), Peterson et al $(2003,2006,2007)$ and by O'Donnel (2007) as clinical filtering tool.

3. Ways of Coping Questionnaire and Charles Carver and colleagues' Coping Orientation of Problem Experience (COPE) (1985). This questionnaire is composed of eight central indexes examining different coping strategies and coping functions with emotion regulation. The coping indexes are (a) confrontive coping (6 items), (b) distancing (6 items), (c) selfcontrolling (7 items), (d) seeking social support (6 items), for example: "Have you been assisted by support outside the family framework - from a psychologist or social worker or from support groups, etc., - and if so, how?"; (e) accepting responsibility (4 items), for example: "Have you accepted the situation, and if so, what helped you do so?"; and (f) escape-avoidance (8 items), (g) painful problem- solving (6 items) and (h) positive reappraisal (7 items). Cronbach's $\alpha$ for these indexes ranges between 0.56 and 0.85 . -For the qualitative study, a semi-structured interview format was used which characterized by flexibility and consisting of open-ended questions from different content areas, Aims and rationale for using semi structured interviews were:

1. To clarify the interviewees' perceptions of their experiences in their own language. Thus, the interview took on the nature of an informal conversation that allowed the interviewer to move among the issues that are judged to be significant, depending on their order of occurrence and the interview's progression (Berg, B.L.1995)

2. The course of the interview and its character are largely dependent on the degree of trust, and the openness between the interviewer and the interviewee and on the nature of their interaction. The interviewer is responsible for leading the interview so in such a way that as many topics as possible are investigated. maximum study of the subject is investigated (Taylor, S.J., \& Bogdan,R. 1984).

3. The dynamics that form between the interviewer and interviewee, shift throughout; the purpose of the interview is to attempt to understand the system of norms, faiths, and motives of the interviewee (Shkedi, A.2003).

\section{Constructing the semi structured interviews}

A semi-structured interview format was used and followed an interview guide (Kvale, 2009), which characterized by flexibility and consisting of questions from different content fields and topics that stemmed from the research questions and their derivatives such as: difficulties and emotions accompanied the process, as well as coping strategies, support systems and continuing / quitting treatments. 
Eight open ended questions were designed as an interview guide which were evaluated in five pilot interviews. Subsequently, minimal changes were made: one more question was added and few more sub-questions, which helped improve the guide.

The interview began with general demographic questions and continued with more focused ones (for example, "Tell me about your desire to bring a child into the world" or "What kind of reactions did you receive from your family, from society, from your spouse / female spouse, from your workplace, and from friends throughout the whole process?" The interviews were tape recorded and literally transformed into text for its analysis.

\section{Ethical Considerations}

Approval has been obtained from the ethics committee of the institution- Maccabi health services. Institutional application number- 0127-17-BBL, after making minor changes in the study protocol, encoding the research questionnaire (for statistical analysis) and signed informed consent form from each patient. The approval was valid for one year. Permission to conduct the study was given by the supervisors at six women's healthcare centers across the country and two medical centers of Assuta hospitals. (women's healthcare centers in: BeerSheba, Haifa, Jerusalem, Modiin, Rishon Lezion, and Tel-Aviv. two medical centers of Assuta in Tel-Aviv and in Rishon Lezion).

Participants who met the study criteria received detailed information about the study's purpose as well as the anonymous questionnaire and were guaranteed that complete confidentiality would be maintained for all the information gathered from them, whether in writing or verbally, and that the research data would only be used for the current study. They were also informed that they could discontinue their participation without having to give any explanation. Patients who agreed to participate and signed the informed consent form were then asked to fill out the questionnaire.

If a problem occurred while a participant was completing the questionnaire, whether in intimacy, medical, or other, the patient was referred to suitable treatment: the treating physician, therapist, family therapy, etc. In such cases, the committee was regularly reported to with respect to those patients' treatments, as required.

The interview did not begin until the interviewer collected from the participant the signed consent form. The participants were also informed of the study's purpose and its importance. The participants were guaranteed that their anonymity would be protected, and that the findings would be archived after completing the study. 


\section{Data collection Procedure}

Data collection was performed from May 2018 to April 2019. Participants who arrived the fertility clinic and met the study's criteria were asked to participate in the study Tracing and recruiting the participants was accomplished through sampling layers, each sampled systematically; the layers were determined according to the research variable that corresponded with the population that was represented in the sample. Locating patients with female spouses was not an easy task since most of them had undergone treatments in private hospitals and there was no direct contact with them. Clinic patients offered me their help locating additional patients and thus opened their community to me. I also approached their centers and even visited them in the effort to broaden my study sample. After obtaining the participants' verbal consent and signing the informed consent form, then they were asked to fill out the questionnaire. once they have completed answering the questionnaire's questions, the questionnaires were returned within envelops.

For the qualitative study, the method of data collection was quota sample - three clusters were selected and in each cluster a convenience sample was conducted until an affixed quota was filled. This method guaranteed representation of every cluster.

prior to beginning of an interview, the participant's consent was needed, and had them sign a consent form to have the interview as well as recording it. The participants were also informed regarding the research purpose and its importance. the participants were promised to maintain anonymity, that there will be no publication and that the findings will be archived upon completion of the study.

The interview took place at the clinic or at the patient's home according to her preference. The duration of the interviews was estimated between 45-60 minutes. Each interview was recorded with the consent of the participant, and general notes were taken during the interview.
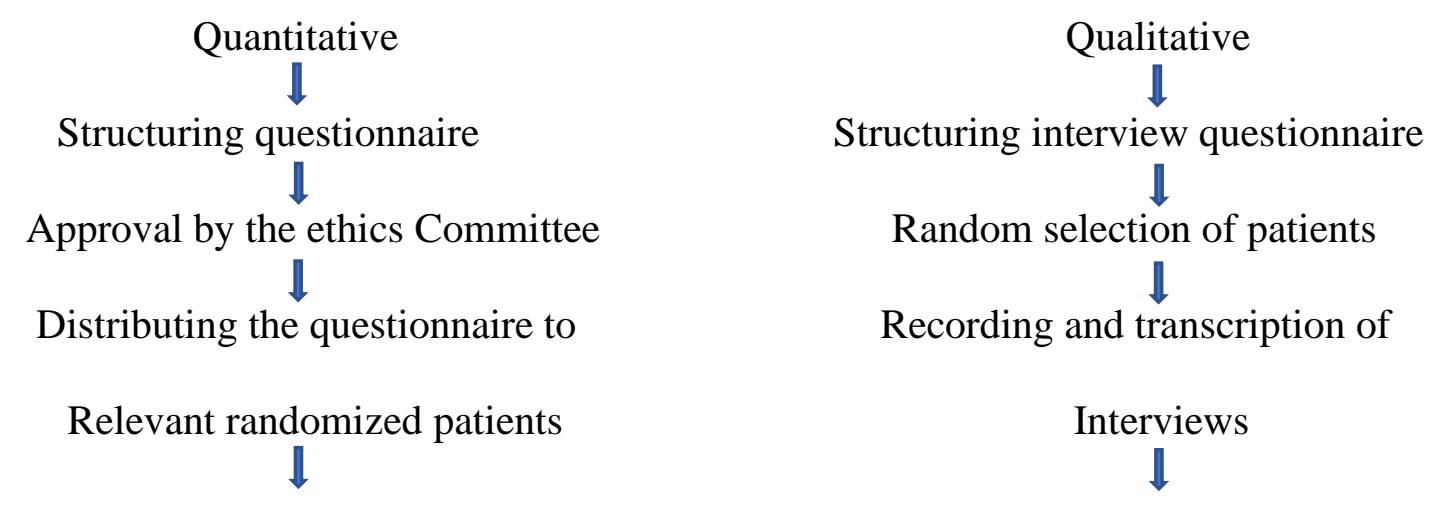
Encoding the questionnaire

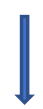

Statistical analysis
Assigning key topics to separate

Categories

$\downarrow$

Creating themes

Figure 2. Research procedure for quantitative and qualitative studies.

\section{Data Analysis}

All the data in the questionnaires were encoded to an Excel table, mostly according to Likert scale. (Example of a question: how does your spouse/ female spouse support you? The answers: 1. Incredibly supportive, 2. Partially supporting, 3. Barely support, 4.not supporting at all, and 5.not relevant - for singles). Some of the answers to the questions were encoded according to answers that patients have given in the questionnaire (example for a question: how do you overcome a failure of a treatment? The answers: 1. Cry/sadness, 2.

Drinking/eating, 3. Go to a movie, 4. Optimistic / carry on, etc.).

Data analysis was conducted using SPSS software. To assess the marital support system for women in fertility treatments, correlations between marital status (single, married and women with female partners) and support systems of women with fertility treatments were computed. To assess correlation with categorical variables (e.g. type of support) Chi Square tests were conducted, this type of analysis compares rates between women from various marital status groups.

* Ethics issues of the study: should a problem appeared during the questionnaire, whether in intimacy, medical or otherwise, the patient was referred to suitable treatment, meaning, a treating doctor, therapist, family therapy, etc. Additionally, the committee was reported on an irregularity when a patient was referred to treatment as required.

A qualitative content analysis approach (Graneheim \& Lundman, 2004) was used to analyze the interview transcripts. Data processing began during data collection to determine when sufficient rich data were collected in relation to the study's purpose (Patton, 2002). Each interview was tape recorded, read multiple times to grasp the entirety of the data collected and transcribed verbatim. the written text was validated against the sound recordings. The text was 
further divided into meaning units - depending on content (Creswell, 2000). These steps were followed during analysis:

1. Interviews were transcribed verbatim after reading multiple times to grasp the entirety of the data; and then the written text was validated against the sound recording.

2. The transcribed text was divided into meaning units - depending on content.

3. The meaning units were then divided into categories and subcategories by scrutinizing their similarities and differences. As illustrated in these steps, analyses of the transcribed interviews in the beginning remained close to the text. Later, the analyses became more interpretative to be able to describe experienced meaning from interviewees' narrations.

4. Then 5 central themes were created.

\section{Chapter 4: Findings}

\section{$\underline{\text { Statistical Analysis }}$}

\section{Study population}

450 women participated in this study. Sample was divided into three groups $-33.3 \%$ were married, $33.3 \%$ were single and $33.3 \%$ had a female partner (figure 3). Age of women ranged between 22 and 45, with an average of 36.41 (Standard Deviation = 5.18). Education distribution showed that $3.8 \%$ finished High-school, $16.7 \%$ had a non-degree certificate, $49.7 \%$ had BA and $29.8 \%$ had MA or higher degree (figure 4).

Among 300 women in this study who had a romantic partner, age of partner ranged between 24 and 54, with an average of 35.89 (Standard Deviation $=5.65$ ). Partners' education distributed with $8 \%$ finished High-school, $24 \%$ had a non-degree certificate, $52 \%$ had BA and $16 \%$ had MA or higher degree (figure 5)

Years of being in relationship ranged between 1 year and 20 years, with an average of 5.77 $(\mathrm{SD}=3.80)$.

About $50 \%$ of women in sample had children while, average number of children was 1.55 $(\mathrm{SD}=0.79)$ (figure 6) 


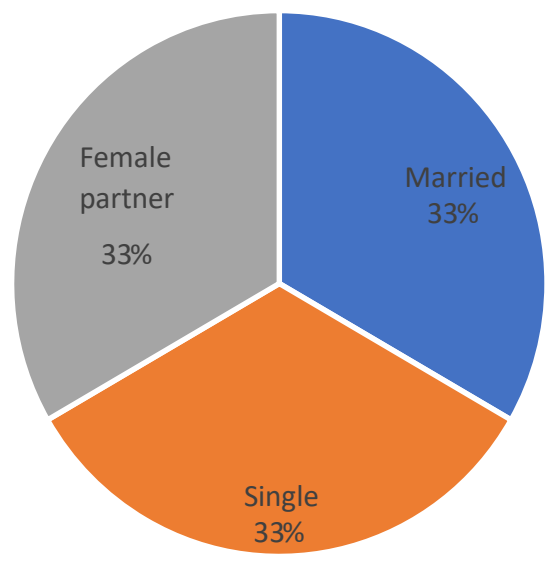

Figure 3: Women in the study by marital status

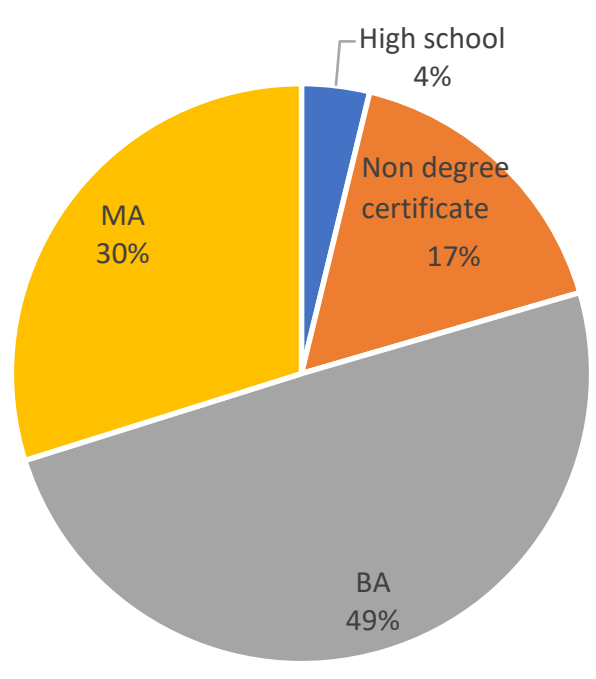

Figure 4: Education of women in the study 


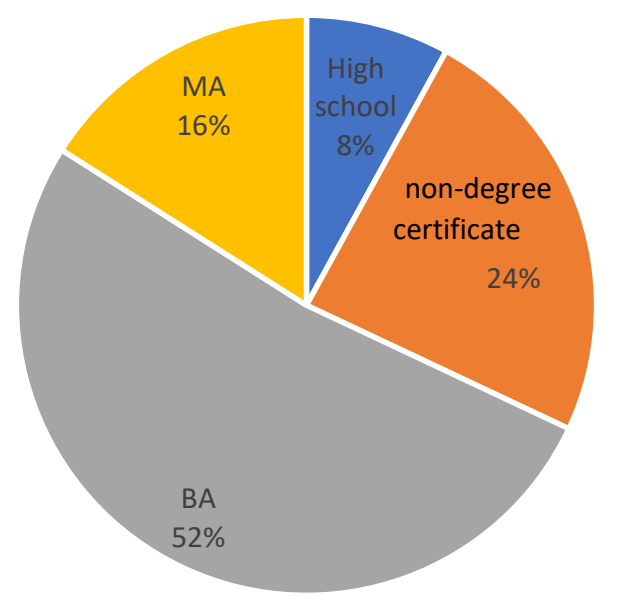

Figure 5: Education of women's partners in the study

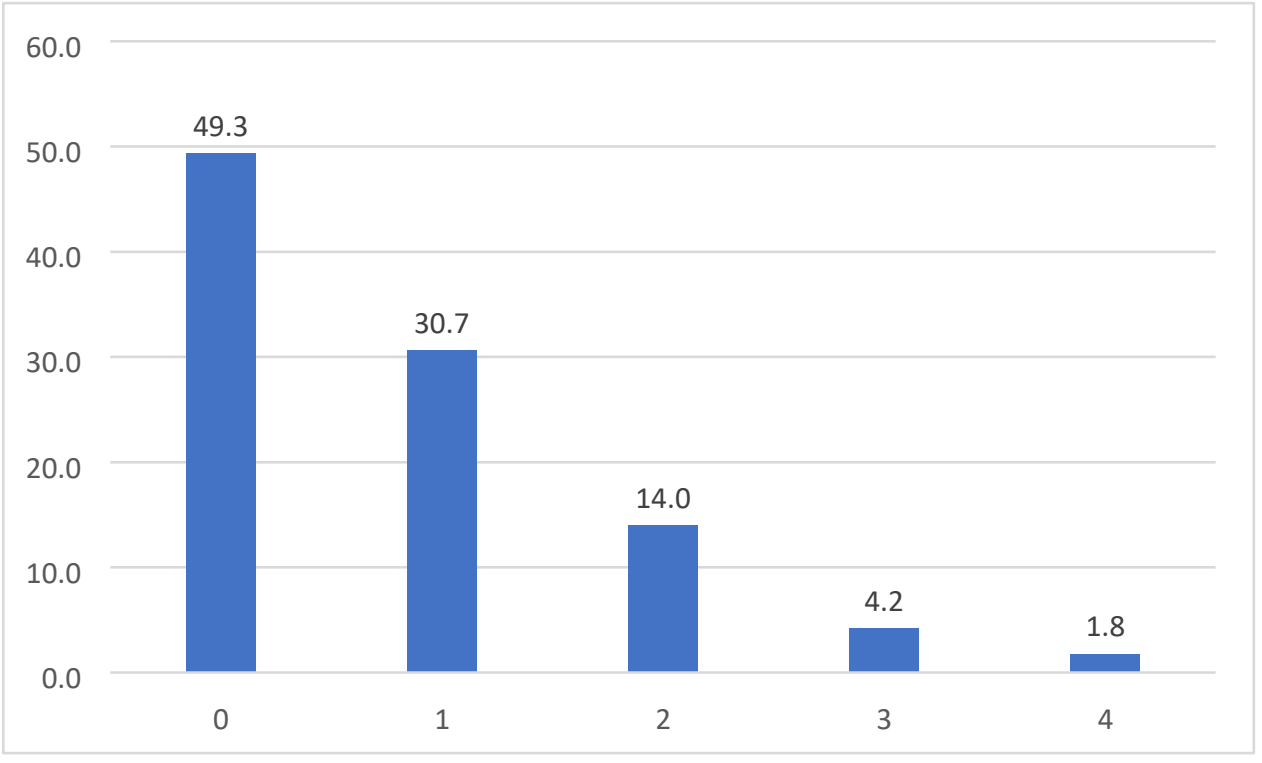

Figure 6: Number of children (\%) 


\section{Results}

In order to examine relationship between marital status and clinical information regarding pregnant attempts, Chi-Square tests were conducted, as shown in tables 4-7. This type of analysis compares rates between women from various marital status groups.

\section{Research Question 1. Does the family structure affect women's coping with fertility treatments?}

To address the first research question, correlations between marital status (single, married and women with female partner) and coping with fertility treatments were computed. To assess correlation with categorical variables (e.g. type of treatments) Chi-Square tests were conducted.

Table 3: Years of fertility treatments by marital status

Married Single $\begin{gathered}\text { Female } \\ \text { partner }\end{gathered}$

\begin{tabular}{lrrrr}
\hline \multirow{2}{*}{$\begin{array}{l}\text { Less than 1 } \\
\text { year }\end{array}$} & 21 & 20 & 45 & 86 \\
\cline { 2 - 5 } $\mathbf{1 - 2}$ & $14.1 \%$ & $13.5 \%$ & $30.2 \%$ & $19.3 \%$ \\
\cline { 2 - 5 } & 70 & 80 & 90 & 340 \\
\hline $\mathbf{2 - 3}$ & $18.3 \%$ & $54.1 \%$ & $60.4 \%$ & $53.6 \%$ \\
\cline { 2 - 5 } & $12.1 \%$ & $20.3 \%$ & $6.7 \%$ & $13.0 \%$ \\
\hline $\mathbf{3 - 4}$ & 13 & 9 & 2 & 24 \\
\hline $\mathbf{4 - 5}$ & $8.7 \%$ & $6.1 \%$ & $1.3 \%$ & $5.4 \%$ \\
\hline & 11 & 3 & 0 & 14 \\
\hline $\begin{array}{l}\text { More than 5 } \\
\text { years }\end{array}$ & $7.4 \%$ & $2.0 \%$ & $0.0 \%$ & $3.1 \%$ \\
\hline
\end{tabular}

As seen in Table 3, most women (73\%) try to get pregnant two years or less. A significant association was found between marital status and years of pregnant attempts $\left(\chi^{2}=63.75, p<\right.$ .001 ), while about $30 \%$ of women with female partner try to get pregnant less than 1 year in comparison with only half of this rate among single women or married women. 
Table 4: Actions during trying to get pregnant by marital status

\begin{tabular}{|c|c|c|c|c|}
\hline & Married & Single & $\begin{array}{l}\text { Female } \\
\text { partner }\end{array}$ & Total \\
\hline \multirow{2}{*}{$\begin{array}{l}\text { Contact a } \\
\text { fertility } \\
\text { specialist }\end{array}$} & 55 & 84 & 120 & 259 \\
\hline & $36.7 \%$ & $56.0 \%$ & $80.0 \%$ & $57.6 \%$ \\
\hline \multirow{2}{*}{$\begin{array}{l}\text { Tests to } \\
\text { investigate } \\
\text { fertility }\end{array}$} & 48 & 27 & 21 & 96 \\
\hline & $32.0 \%$ & $18.0 \%$ & $14.0 \%$ & $21.3 \%$ \\
\hline \multirow[t]{2}{*}{ IVF } & 47 & 38 & 8 & 93 \\
\hline & $31.3 \%$ & $25.3 \%$ & $5.3 \%$ & $20.7 \%$ \\
\hline \multirow{2}{*}{$\begin{array}{l}\text { Alternative } \\
\text { medicine }\end{array}$} & 0 & 1 & 1 & 2 \\
\hline & $0.0 \%$ & $0.7 \%$ & $0.7 \%$ & $0.4 \%$ \\
\hline
\end{tabular}

As seen in Table 4, a significant association was found between marital status and actions during trying to get pregnant $\left(\chi^{2}=42.12, \mathrm{p}<.001\right)$. Most women $(57.6 \%)$ consulted fertility specialists, while rates were different by marital status. $80 \%$ of women with female partner contacted fertility specialists, only $56.0 \%$ and $36.7 \%$ did it among single and married women, respectively. In addition, $21.3 \%$ of women reported they conducted tests to investigate fertility, while rates were different by marital status. $14 \%$ of women with female partner investigated fertility, $18.0 \%$ and $32.0 \%$ did it among single and married women respectively. About $20.7 \%$ of women conducted IVF treatment, while rates were different by marital status. It was found that most women conducted IVF treatment were married (31.3\%) or single (25.3\%). Only 5.3\% of women with female partner did IVF.

Table 5: Testing following attempts to get pregnant by marital status

\begin{tabular}{lrrrr}
\hline & Married Single & \multicolumn{3}{c}{$\begin{array}{c}\text { Female } \\
\text { partner }\end{array}$} \\
\hline PAP test & 95 & 75 & 69 & 239 \\
\cline { 2 - 5 } & $63.3 \%$ & $50.0 \%$ & $46 \%$ & $53.1 \%$ \\
\hline Hysterosalpingography & 53 & 31 & 21 & 105 \\
\cline { 2 - 5 } & $35.3 \%$ & $20.7 \%$ & $14.0 \%$ & $23.3 \%$ \\
\hline Hysteroscopy & 6 & 22 & 6 & 34 \\
\cline { 2 - 5 } & $4.0 \%$ & $14.7 \%$ & $4.0 \%$ & $7.6 \%$ \\
\hline
\end{tabular}




\begin{tabular}{lrrrr}
\hline Blood tests & 11 & 12 & 18 & 41 \\
\cline { 2 - 5 } & $7.3 \%$ & $8.0 \%$ & $12.0 \%$ & $9.1 \%$ \\
\hline $\begin{array}{l}\text { Ultrasound tracking } \\
\text { follicles }\end{array}$ & 1 & 0 & 0 & 1 \\
\cline { 2 - 5 } & $0.7 \%$ & $0.0 \%$ & $0.0 \%$ & $0.2 \%$ \\
\hline $\begin{array}{l}\text { Breast / } \\
\text { mammography }\end{array}$ & 5 & 6 & 5 & 16 \\
\hline $\begin{array}{l}\text { ECG } \\
\text { (Electrocardiography) }\end{array}$ & $3.3 \%$ & $4.0 \%$ & $3.3 \%$ & $3.6 \%$ \\
\hline
\end{tabular}

As shown in Table 5, a significant association was found between marital status and testing following attempts to get pregnant $\left(\chi^{2}=39.54, \mathrm{p}<.001\right)$. About half of total sample $(53.1 \%)$ conducted PAP tests to get pregnant, while higher percentage was found among married women $(63.3 \%)$ in comparison with single women (50\%) and women with female spouse (46\%). In addition, about one quarter (23.3\%) conducted hysterosalpingography, while higher percentage was found among married women (35.3\%) in comparison with single women $(20.7 \%)$ and women with female partners (14\%).

7.6\% of total sample had Hysteroscopy, with higher percentage among single women $(14.7 \%)$. In addition, blood tests were conducted by $9.1 \%$ of total sample, with higher percentage among women with female partners $(12 \%)$.

Finally, mammography and ECG were conducted by $3.6 \%$ and $3.3 \%$ of total sample, respectively.

Table 6: Treatments following attempts to get pregnant by marital status

\begin{tabular}{|c|c|c|c|c|}
\hline & Married & Single & $\begin{array}{l}\text { Female } \\
\text { partner }\end{array}$ & Total \\
\hline \multirow{2}{*}{$\begin{array}{l}\text { IVF / frozen cycle } \\
\text { treatment }\end{array}$} & 24 & 35 & 7 & 66 \\
\hline & $16.0 \%$ & $23.3 \%$ & $4.7 \%$ & $14.7 \%$ \\
\hline \multirow{2}{*}{$\begin{array}{l}\text { Clomiphene citrate } \\
\text { IUI }\end{array}$} & 28 & 5 & 61 & 94 \\
\hline & $18.7 \%$ & $3.3 \%$ & $40.7 \%$ & $20.9 \%$ \\
\hline \multirow[t]{2}{*}{ Hormonal therapy } & 98 & 110 & 82 & 290 \\
\hline & $65.3 \%$ & $73.3 \%$ & $54.7 \%$ & $64.4 \%$ \\
\hline
\end{tabular}

As shown in Table 6, a significant association was found between marital status and treatments following pregnant attempts $\left(\chi^{2}=29.23, \mathrm{p}<.001\right)$. Most women in total sample $(64.4 \%)$ had 
conducted hormonal therapy while single women conducted more treatments $(73.3 \%)$ in comparison with married women (65.3\%) and women with female partner (54.7\%). In addition, about $14.7 \%$ of total sample conducted IVF treatments (frozen cycle treatment), with higher percentage among single women (23.3\%) in comparison with married women (16\%) and women with female partner $(4.7 \%)$.

Finally, $20.9 \%$ of women in total sample reported they conducted Clomiphene citrate+ IUI, while women with female partner reported on the highest rate of this type of treatment $(40.7 \%)$ relatively to married women $(18.7 \%)$ and single women $(3.3 \%)$.

\section{Research Question 2. Is the marital support system for women in fertility treatments necessarily more stable than that of female partner and single women?}

To address the second research question, correlations between marital status (single, married and women with female partners) and support systems of women with fertility treatments were computed. To assess correlation with categorical variables (e.g. type of support) Chi Square tests were conducted. To assess differences between groups in continuous variables (the degree women feel support), Analysis of Variance were computed. In addition, to examine correlation between marital status and support of relatives and partner, Pearson correlations were conducted.

As shown in Table 7, a significant difference was found, while married women reported the highest level of family support during pregnant attempts, in comparison with single women or women with female partners $(\mathrm{F}(2,446)=8.57, \mathrm{p}<.05)$. In addition, results showed that women with female partner reported higher partners' support in comparison with married women (F $(1,298)=121.57, \mathrm{p}<.01)$.

Table 7: Means of family support and partner's support levels among women by marital status:

\begin{tabular}{lrrrr}
\hline $\begin{array}{c}\text { Marital } \\
\text { status }\end{array}$ & \multicolumn{2}{c}{ Family support } & \multicolumn{2}{c}{ Partner's support } \\
\hline Mean & $\begin{array}{c}\text { Std. } \\
\text { Deviation }\end{array}$ & Mean & $\begin{array}{c}\text { Std. } \\
\text { Deviation }\end{array}$ \\
\hline Married & 4.282 & 1.987 & 3.287 & 0.780 \\
\hline Single & 3.927 & 1.754 & - & - \\
\hline
\end{tabular}




\begin{tabular}{lcccc}
\hline $\begin{array}{l}\text { Female } \\
\text { partner }\end{array}$ & 3.967 & 1.640 & 3.993 & 0.082 \\
\hline Total & 4.058 & 1.802 & 3.640 & 0.657 \\
\hline
\end{tabular}

Assessing Pearson correlations between level of family support and other demographic variables did not yield significant correlations (table 8). However, weak negative correlations were found between women's education and partner's age, while lower education and younger partner predicted high family support.

Examining correlations with partner support revealed negative correlations with education, partner's age, and years of relationship. Meaning that lower education, younger partner, and low number of relationship years are correlated with higher partner support during attempts to get pregnant.

Table 8: Correlations with family and partner support

\begin{tabular}{lcc}
\hline Variable & Family support & Partner support \\
\hline Age & .019 & .028 \\
\hline Education & -.102 & $-.134^{*}$ \\
\hline Partner's age & -.109 & $-.132^{*}$ \\
\hline Partner's education & .029 & .101 \\
\hline Years of relationship & .064 & $-.334^{* *}$ \\
\hline Number of kids & .021 & .029 \\
\hline
\end{tabular}

Table 9: Mental treatment following attempts to get pregnant by marital status

\begin{tabular}{|c|c|c|c|c|}
\hline & Married & Single & $\begin{array}{l}\text { Female } \\
\text { partner }\end{array}$ & Total \\
\hline \multirow{2}{*}{$\begin{array}{l}\text { Did not } \\
\text { need } \\
\text { mental } \\
\text { assistance }\end{array}$} & 51 & 17 & 142 & 210 \\
\hline & $34.0 \%$ & $11.3 \%$ & $94.7 \%$ & $46.7 \%$ \\
\hline \multirow[t]{2}{*}{ Therapist } & 38 & 63 & 3 & 104 \\
\hline & $25.3 \%$ & $42.0 \%$ & $2.0 \%$ & $23.1 \%$ \\
\hline \multirow{2}{*}{$\begin{array}{l}\text { Support } \\
\text { groups }\end{array}$} & 51 & 58 & 5 & 114 \\
\hline & $34.0 \%$ & $38.7 \%$ & $3.3 \%$ & $25.3 \%$ \\
\hline \multirow{2}{*}{$\begin{array}{l}\text { Alternative } \\
\text { treatment }\end{array}$} & 10 & 12 & 0 & 22 \\
\hline & $6.7 \%$ & $8.0 \%$ & $0.0 \%$ & $4.9 \%$ \\
\hline
\end{tabular}


As shown in Table 9, a significant association was found between marital status and mental assistance following pregnant attempts $\left(\chi^{2}=47.29, \mathrm{p}<.001\right)$. About half of total sample $(46.7 \%)$ reported they did not need any mental assistance (external support), while almost all women with female partner did not need external help (94.7\%) in comparison with single women $(11.3 \%)$ and married women (34\%). In addition, $23.1 \%$ of total sample received therapist help (psychologist or social worker) while higher percentage of single women were treated by therapist (42\%) in comparison with married women $(25.3 \%)$ and women with female partner $(2 \%)$.

About $25 \%$ of total sample consulted with support groups, with higher percentage among married women $(34 \%)$ and single women $(38.7 \%)$. Only $5 \%$ had alternative treatments.

Table 10- Feelings following attempts to get pregnant by marital status

\begin{tabular}{lc}
\hline Feeling & Percent \\
\hline Frustration & 32.0 \\
\hline Stress & 16.4 \\
\hline Pain & 14.0 \\
\hline Disappointment & 10.4 \\
\hline Lack of self-control & 7.3 \\
\hline Mood swing & 5.1 \\
\hline Anger & 4.9 \\
\hline Guilt & 3.6 \\
\hline Fear & 3.3 \\
\hline Hope & 1.6 \\
\hline Sadness & 0.9 \\
\hline Loneliness & 0.4 \\
\hline
\end{tabular}

As shown in Table 10, the most dominant feeling reported by women in this study following attempts to get pregnant is frustration (32\%). In addition, women reported strong feelings of stress $(16.4 \%)$, pain (14\%) and disappointment (10.4\%). It is interesting to note that the least experienced feelings are hope (1.6\%), sadness $(0.9 \%)$ and loneliness $(0.4 \%)$.

Table 11. Sharing feelings with other people by marital status

Married Single $\begin{gathered}\text { Female } \\ \text { partner }\end{gathered}$ Total




\begin{tabular}{lrrrr}
\hline Do not share anyone & 22 & 43 & 5 & 70 \\
\cline { 2 - 5 } & $14.7 \%$ & $28.7 \%$ & $3.3 \%$ & $15.6 \%$ \\
\hline Do not share everything & 23 & 16 & 17 & 56 \\
\cline { 2 - 5 } Only spouse & $15.3 \%$ & $10.7 \%$ & $11.3 \%$ & $12.4 \%$ \\
\hline Only a family member & 27 & 0 & 123 & 150 \\
\hline Close friends & $18.0 \%$ & 0 & $82.0 \%$ & $32.9 \%$ \\
\hline With everyone & $18.0 \%$ & $27.3 \%$ & $0.7 \%$ & $15.3 \%$ \\
\hline & 33 & 43 & 0 & 76 \\
\hline & $22.0 \%$ & $28.7 \%$ & $0.0 \%$ & $16.9 \%$ \\
\hline
\end{tabular}

As shown in Table 11, a significant association was found between marital status and sharing feelings with social environment following pregnant attempts $(\chi 2=35.13, p<.001)$.

$32.9 \%$ of women reported they share their emotions only with their spouses, stronger preference of women with female partner (82\%). In addition, $15.3 \%$ of women share only with a family member, with highest rate among single women (27.3\%). Another $16.9 \%$ share with close friends, while this channel is most preferable for married (22\%) and single (28.7\%) women. In order to examine differences at effect of reproductive treatments on relationship with partner, independent t-tests were conducted. Results show that women with female partner reported higher partner's involvement following reproductive treatments in comparison with married women $(\mathrm{t}=13.26, \mathrm{p}<.01)$. However, married women reported higher relationship satisfaction following reproductive treatments $(\mathrm{t}=37.23, \mathrm{p}<.01)$. (table 12).

Table 12: The effect of reproductive treatments on partner's involvement and relationship satisfaction

\begin{tabular}{lcccc}
\hline & \multicolumn{2}{c}{ Partner's involvement } & \multicolumn{2}{c}{ Relationship satisfaction } \\
\hline & Mean & Std. Deviation & Mean & Std. Deviation \\
\hline Married & 3.033 & 0.855 & 4.820 & 0.464 \\
\hline Female partner & 3.980 & 0.182 & 2.000 & 0.803
\end{tabular}

Participants were asked regarding their coping strategies as a couple following failure of reproductive treatments. 
Table 13: coping strategies as a couple following failure of reproductive treatments

\begin{tabular}{lc} 
Feeling & Percent \\
\hline Shared recreation & 19.9 \\
\hline Disappointment & 19.6 \\
\hline Optimism & 16.3 \\
\hline Expectation & 13.3 \\
\hline Everyone faces his own way & 12.6 \\
\hline Listening & 9.3 \\
\hline Pain & 9.0 \\
\hline
\end{tabular}

As shown in Table 13, the most common coping strategy of both couples in the sample following failure of reproductive treatments are: Shared recreation (19.9\%), Disappointment $(19.6 \%)$ but also Optimism (16.3\%). On the other hand, the less common feeling of women after failure of reproductive treatments is pain $(9 \%)$.

It is interesting to note that among married women $12.6 \%$ reported that both women and their spouses faced their feelings in different ways (their own way).

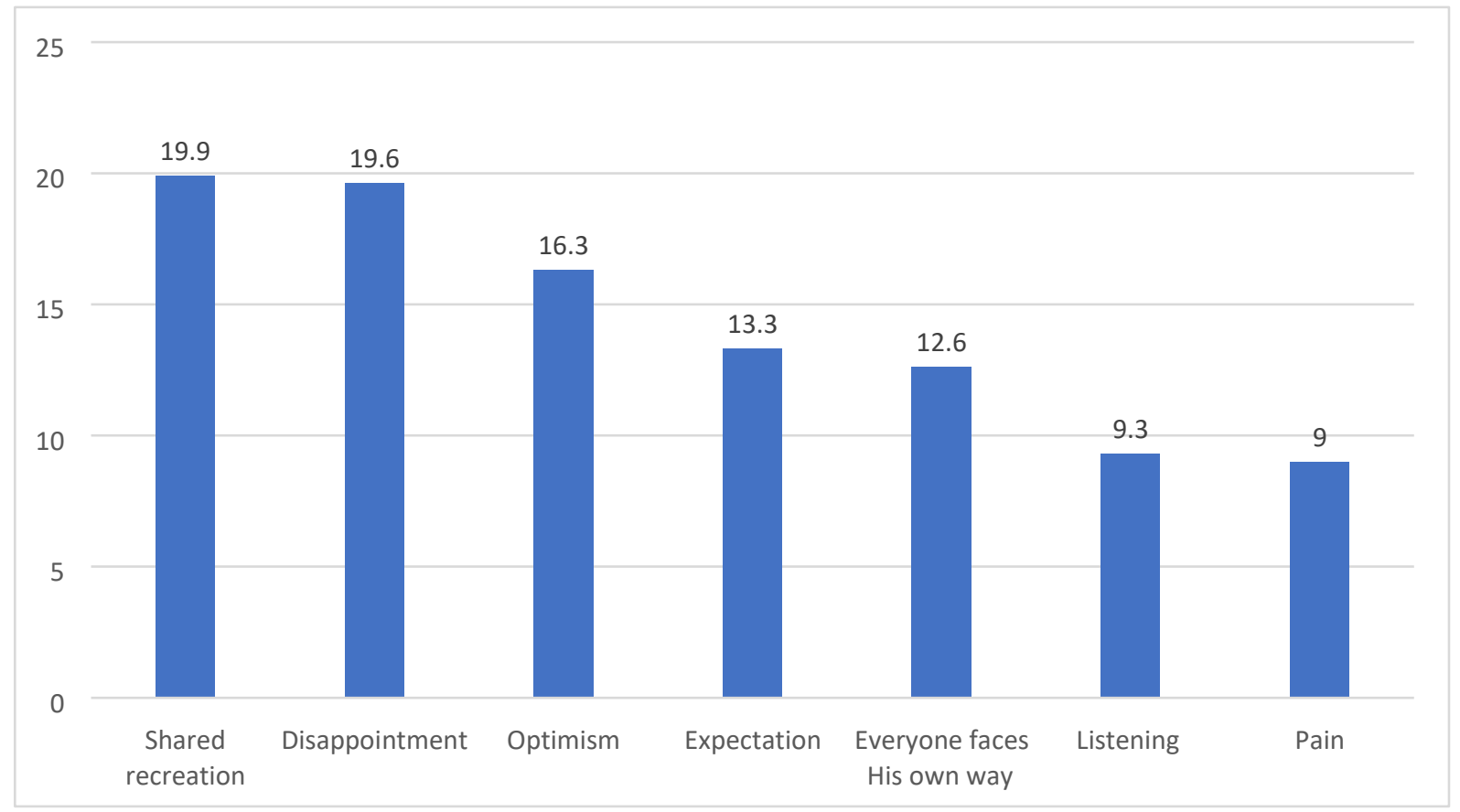

Figure 7: Common coping strategy of couples following failure of reproductive treatments

In order to examine types of family's support women get by marital status, Chi-Square test was conducted. 
Table 14: type of family support by marital status

\begin{tabular}{lcccc}
\hline & Married & Single & $\begin{array}{c}\text { Female } \\
\text { partner }\end{array}$ & Total \\
\hline Encouragement & 45 & 30 & 25 & 100 \\
\cline { 2 - 5 } / empathy & $30 \%$ & $20 \%$ & $28.7 \%$ & $22.3 \%$ \\
\hline Listening & 19 & 11 & 14 & 44 \\
\cline { 2 - 5 } & $12.7 \%$ & $7.4 \%$ & $9.3 \%$ & $9.8 \%$ \\
\hline Giving advice & 10 & 22 & 10 & 42 \\
\hline \multirow{nyyyy}{*}{ Reinforcements } & $6.7 \%$ & $14.8 \%$ & $6.7 \%$ & $9.4 \%$ \\
\cline { 2 - 5 } & $17.3 \%$ & $11.3 \%$ & $5.3 \%$ & $11.4 \%$ \\
\hline No support & 50 & 70 & 93 & 213 \\
\cline { 2 - 5 } & $17.5 \%$ & $46.6 \%$ & $62 \%$ & $47.1 \%$ \\
\hline
\end{tabular}

As shown in Table 14, a significant association was found between marital status and type of family's support $(\chi 2=26.16, p<.001)$. Among all women, encouragement and empathy were rated as the most common type of emotional support (22.3\%), while married women report the highest rate of empathy (30\%). In addition, 9.8\% of women reported on listening and conversations as another type of support, while married women enjoy the most this kind of support $(12.7 \%)$. It is interesting to note that about half of sample $(47.1 \%)$ report they have no emotional support from close family, with higher rate among women with female partner (62\%) and single women $(46.6 \%)$.

Table 15: Coping strategies by marital status

\begin{tabular}{|c|c|c|c|c|}
\hline & Married & Single & $\begin{array}{l}\text { Female } \\
\text { partner }\end{array}$ & Total \\
\hline \multirow[t]{2}{*}{ Crying } & 35 & 29 & 25 & 89 \\
\hline & $23.3 \%$ & $19.3 \%$ & $16.8 \%$ & $19.8 \%$ \\
\hline \multirow{2}{*}{$\begin{array}{l}\text { Drinking, } \\
\text { eating, and } \\
\text { smoking }\end{array}$} & 4 & 8 & 9 & 21 \\
\hline & $2.7 \%$ & $5.3 \%$ & $6.0 \%$ & $4.7 \%$ \\
\hline \multirow[t]{2}{*}{ Shopping } & 7 & 2 & 4 & 13 \\
\hline & $4.7 \%$ & $1.3 \%$ & $2.7 \%$ & $2.9 \%$ \\
\hline \multirow[t]{2}{*}{ Optimism } & 47 & 44 & 47 & 138 \\
\hline & $31.3 \%$ & $29.3 \%$ & $31.5 \%$ & $30.7 \%$ \\
\hline \multirow[t]{2}{*}{ Meditation } & 13 & 6 & 22 & 41 \\
\hline & $8.7 \%$ & $4.0 \%$ & $14.8 \%$ & $9.1 \%$ \\
\hline
\end{tabular}




\begin{tabular}{lrrrr}
\hline Routine & 11 & 6 & 9 & 26 \\
\cline { 2 - 5 } & $7.3 \%$ & $4.0 \%$ & $6.0 \%$ & $5.8 \%$ \\
\hline $\begin{array}{l}\text { Do not } \\
\text { overcome }\end{array}$ & 33 & 55 & 33 & 121 \\
\cline { 2 - 5 } & $22.0 \%$ & $36.7 \%$ & $22.1 \%$ & $26.9 \%$ \\
\hline
\end{tabular}

As shown in Table 15, a significant association was found between marital status and coping strategies $(\chi 2=37.15, \mathrm{p}<.001)$. Among all women, the most common coping strategy to failure in reproductive treatment is being optimistic (30.7\%). However, $19.8 \%$ of women report they cry and get sad, while married (23.3\%) and single women (19.8\%) cry the most. Other coping strategies were drinking, eating, and smoking (4.7\%), shopping (2.9\%), meditation $(9.1 \%)$ and keeping routine $(5.8 \%)$.

To understand how the treatments, affect lives of the women in the sample, according to their marital status, Analysis of Variance procedures were conducted as shown in table 16.

Table 16: Differences in effects of treatments by marital status

\begin{tabular}{|c|c|c|c|c|c|}
\hline Marital & & Mean & $\begin{array}{c}\text { Std. } \\
\text { Deviation }\end{array}$ & $\mathbf{F}$ & p \\
\hline \multirow{4}{*}{$\begin{array}{l}\text { Effects of } \\
\text { social } \\
\text { encounters }\end{array}$} & Married & 2.32 & 0.922 & 3.31 & .012 \\
\hline & Single & 2.34 & 0.784 & & \\
\hline & $\begin{array}{l}\text { Female } \\
\text { partner }\end{array}$ & 2.11 & 0.891 & & \\
\hline & Total & 2.26 & 0.872 & & \\
\hline \multirow{4}{*}{$\begin{array}{l}\text { Effects of } \\
\text { treatments } \\
\text { on routine }\end{array}$} & Married & 3.86 & 1.099 & 14.74 & $<.001$ \\
\hline & Single & 4.02 & 0.847 & & \\
\hline & $\begin{array}{l}\text { Female } \\
\text { partner }\end{array}$ & 3.45 & 0.863 & & \\
\hline & Total & 3.78 & 0.972 & & \\
\hline \multirow[t]{5}{*}{ Faith } & Married & 3.05 & 1.706 & 3.09 & .042 \\
\hline & Single & 3.49 & 1.483 & & \\
\hline & $\begin{array}{l}\text { Female } \\
\text { partner }\end{array}$ & 3.19 & 1.517 & & \\
\hline & Total & 3.24 & 1.579 & & \\
\hline & Married & 3.12 & 1.451 & 0.591 & .554 \\
\hline
\end{tabular}




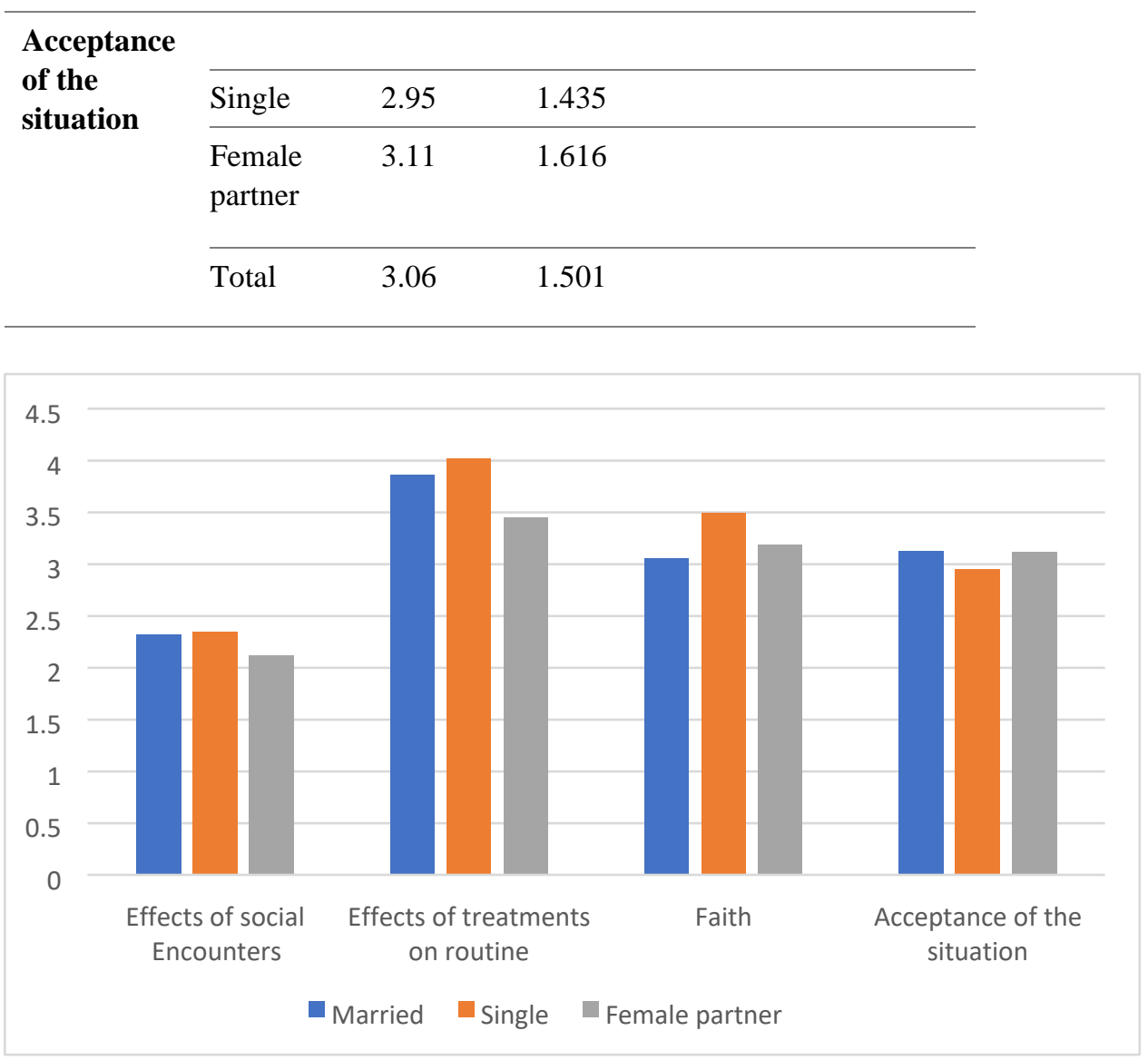

Figure 8: Differences in effects of treatments by marital status

It was found that social encounters affected most of the women in the sample during their treatments with a slightly higher tendency in single women. It was also found that the daily routine of single women is slightly more affected than for married women and women with female partner. single women believe that it was a matter of fate while married women and women with a female partner have accepted the situation somewhat more than single women

\section{Research Question 3. Does the type of treatment influence how to deal with fertility}

\section{treatments?}

To address third question, Analysis of Variances procedures were conducted to assess differences between types of treatments and emotionally coping with their situation.

Table 17: Differences in effects of treatments by type of treatment

\begin{tabular}{llcccc}
\hline Marital & & Mean & $\begin{array}{c}\text { Std. } \\
\text { Deviation }\end{array}$ & F & p \\
\hline $\begin{array}{l}\text { Effects of } \\
\text { treatments } \\
\text { on routine }\end{array}$ & $\begin{array}{l}\text { IVF / } \\
\text { frozen } \\
\text { cycle } \\
\text { treatment }\end{array}$ & 4.015 & 0.886 & & \\
\hline
\end{tabular}




\begin{tabular}{|c|c|c|c|c|c|}
\hline & $\begin{array}{l}\text { Clomiphene } \\
\text { citrate + } \\
\text { IUI }\end{array}$ & 3.585 & 0.955 & & \\
\hline & $\begin{array}{l}\text { Hormonal } \\
\text { therapy }\end{array}$ & 3.783 & 0.987 & & \\
\hline & Total & 3.776 & 0.972 & & \\
\hline \multirow[t]{4}{*}{ Faith } & $\begin{array}{l}\text { IVF / } \\
\text { frozen } \\
\text { cycle } \\
\text { treatment }\end{array}$ & 3.333 & 1.543 & 1.23 & .391 \\
\hline & $\begin{array}{l}\text { Clomiphene } \\
\text { citrate }+ \\
\text { IUI }\end{array}$ & 3.223 & 1.539 & & \\
\hline & $\begin{array}{l}\text { Hormonal } \\
\text { therapy }\end{array}$ & 3.231 & 1.604 & & \\
\hline & Total & 3.244 & 1.579 & & \\
\hline \multirow[t]{4}{*}{$\begin{array}{l}\text { Acceptance } \\
\text { of the } \\
\text { situation }\end{array}$} & $\begin{array}{l}\text { IVF / } \\
\text { frozen } \\
\text { cycle } \\
\text { treatment }\end{array}$ & 3.030 & 1.488 & 1.09 & .524 \\
\hline & $\begin{array}{l}\text { Clomiphene } \\
\text { citrate }+ \\
\text { IUI }\end{array}$ & 3.053 & 1.602 & & \\
\hline & $\begin{array}{l}\text { Hormonal } \\
\text { therapy }\end{array}$ & 3.072 & 1.476 & & \\
\hline & Total & 3.062 & 1.501 & & \\
\hline
\end{tabular}

As shown in Table 17 a significant difference was found between women according type of treatment. Women who conducted IVF treatment were significantly more emotional affected in comparison with women who conducted IUI of hormonal therapy. However, level of faith or acceptance of the situation were not different between women according to type of treatment. 


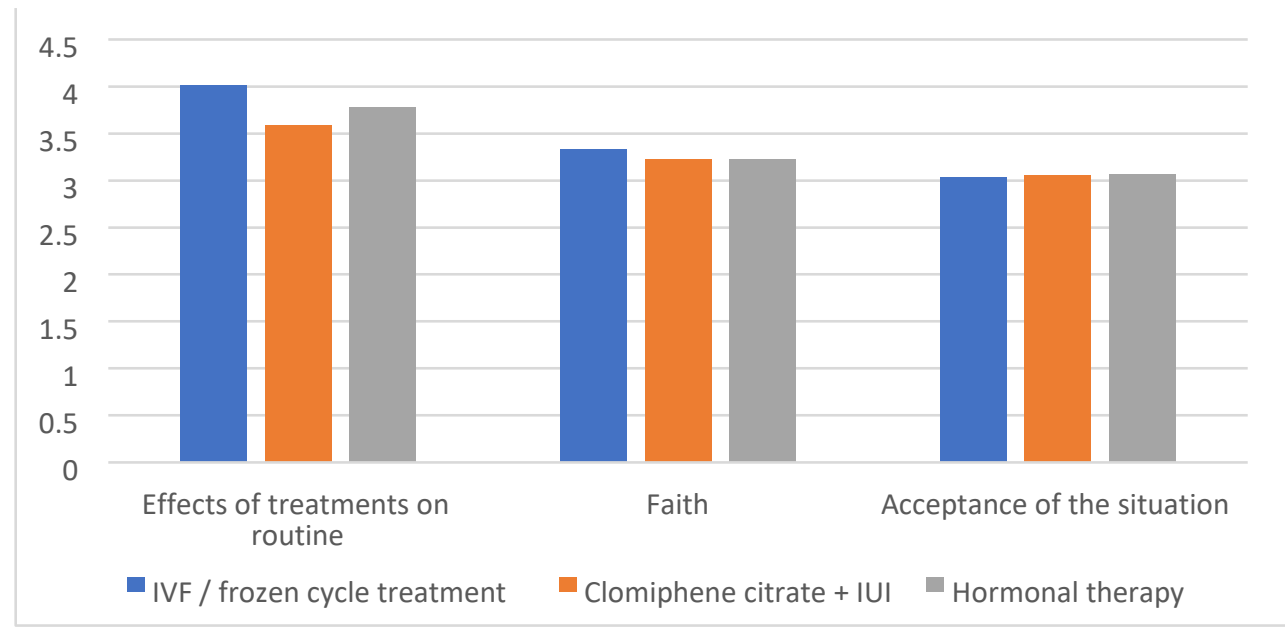

Figure 9: Differences in effects of treatments by type of treatment

In addition, correlations between type of treatment and coping strategies were calculated using Chi-Square tests.

Table 18: Differences in coping strategies by type of treatment

\begin{tabular}{|c|c|c|c|c|}
\hline & $\begin{array}{c}\text { IVF / } \\
\text { frozen } \\
\text { cycle } \\
\text { treatment }\end{array}$ & $\begin{array}{l}\text { Clomiphene } \\
\text { citrate } \\
\text { + IUI }\end{array}$ & $\begin{array}{l}\text { Hormonal } \\
\text { therapy }\end{array}$ & \\
\hline \multirow[t]{2}{*}{ Crying } & 15 & 23 & 51 & 89 \\
\hline & $22.7 \%$ & $24.7 \%$ & $17.6 \%$ & $19.8 \%$ \\
\hline \multirow{2}{*}{$\begin{array}{l}\text { Drinking, } \\
\text { eating and } \\
\text { smoking }\end{array}$} & 0 & 3 & 18 & 21 \\
\hline & $0.0 \%$ & $3.2 \%$ & $6.2 \%$ & $4.7 \%$ \\
\hline \multirow[t]{2}{*}{ Shopping } & 4 & 2 & 7 & 13 \\
\hline & $6.1 \%$ & $2.2 \%$ & $2.4 \%$ & $2.9 \%$ \\
\hline \multirow[t]{2}{*}{ Optimism } & 18 & 24 & 96 & 138 \\
\hline & $27.3 \%$ & $25.8 \%$ & $33.1 \%$ & $30.7 \%$ \\
\hline \multirow[t]{2}{*}{ Meditation } & 4 & 14 & 23 & 41 \\
\hline & $6.1 \%$ & $15.1 \%$ & $7.9 \%$ & $9.1 \%$ \\
\hline \multirow[t]{2}{*}{ Routine } & 5 & 5 & 16 & 26 \\
\hline & $7.6 \%$ & $5.4 \%$ & $5.5 \%$ & $5.8 \%$ \\
\hline \multirow{2}{*}{$\begin{array}{l}\text { Do not } \\
\text { overcome }\end{array}$} & 20 & 22 & 79 & 121 \\
\hline & $30.3 \%$ & $23.7 \%$ & $27.2 \%$ & $26.9 \%$ \\
\hline
\end{tabular}

As shown in Table 18, among women who conducted IVF, they mostly cried (22.7\%), or did not overcome the crisis (30.3\%). However, $27.3 \%$ of women reported they were optimistic. 
Similarly, among women who conducted IUI, $24.7 \%$ reported they mostly cry, and $23.7 \%$ report they did not overcome the crisis. However, 25.8\% of women reported they were optimistic. Finally, among women who received hormonal therapy, level of optimisms was the highest (33.1\%).

\section{Research Question 4. Is the coping process different to the extent that the couple already}

\section{has children?}

To address fourth question, independent t-tests procedures were conducted in order to assess differences between women with and without children on emotionally coping with their situation.

Table 19: Differences in effects of treatments by children

\begin{tabular}{|c|c|c|c|c|c|}
\hline & & Mean & $\begin{array}{c}\text { Std. } \\
\text { Deviation }\end{array}$ & $\mathbf{t}$ & p \\
\hline \multirow{2}{*}{$\begin{array}{l}\text { Effects of } \\
\text { treatments } \\
\text { on routine }\end{array}$} & $\begin{array}{l}\text { No } \\
\text { children }\end{array}$ & 3.838 & 0.947 & 1.32 & .180 \\
\hline & $\begin{array}{l}\text { With } \\
\text { children }\end{array}$ & 3.115 & 0.994 & & \\
\hline \multirow[t]{2}{*}{ Faith } & $\begin{array}{l}\text { No } \\
\text { children }\end{array}$ & 3.446 & 1.496 & 2.71 & .007 \\
\hline & $\begin{array}{l}\text { With } \\
\text { children }\end{array}$ & 3.048 & 1.635 & & \\
\hline \multirow{2}{*}{$\begin{array}{l}\text { Acceptance } \\
\text { of the } \\
\text { situation }\end{array}$} & $\begin{array}{l}\text { No } \\
\text { children }\end{array}$ & 3.126 & 1.468 & 0.901 & .374 \\
\hline & $\begin{array}{l}\text { With } \\
\text { children }\end{array}$ & 3.000 & 1.534 & & \\
\hline
\end{tabular}

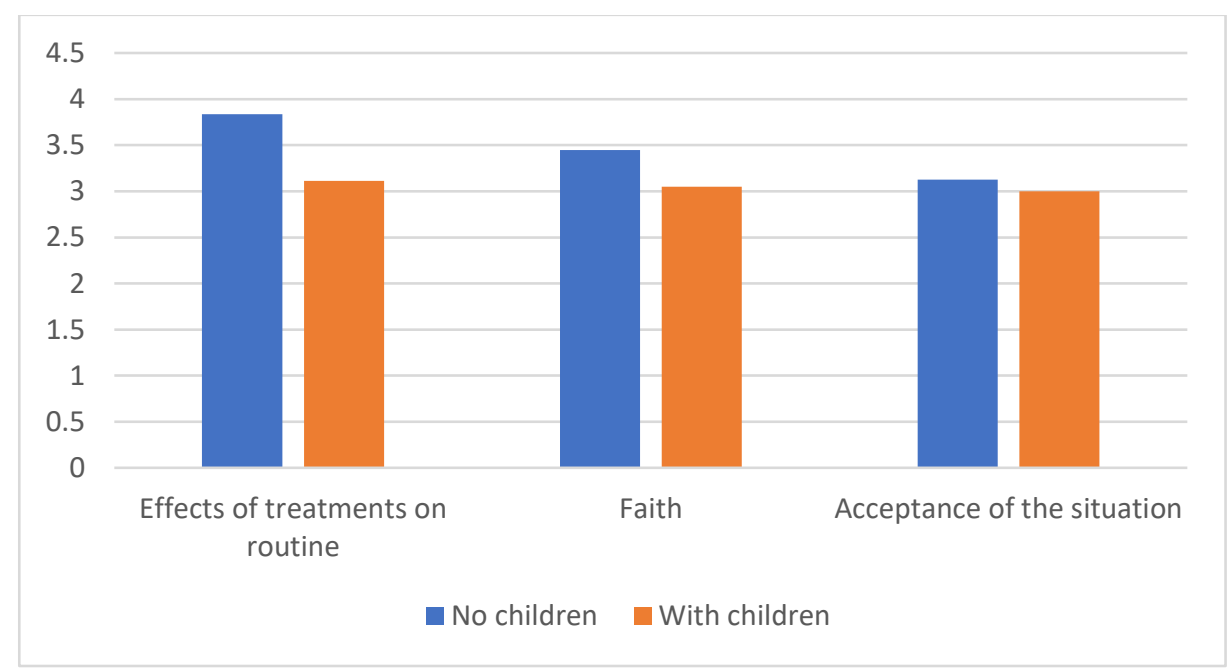

Figure 10: Differences in effects of treatments by children 
As shown in Table 19, women who have no children are more negatively influenced in comparison with women with children.

In addition, women with no children believe that difficulties in getting pregnant is a matter of faith, more than women with children.

Table 20: Differences between women with and without children in coping strategies

\begin{tabular}{lccc}
\hline & $\begin{array}{c}\text { No } \\
\text { children }\end{array}$ & $\begin{array}{c}\text { With } \\
\text { children }\end{array}$ & Total \\
\hline Crying & 45 & 44 & 89 \\
\cline { 2 - 4 } & $20.3 \%$ & $19.4 \%$ & $19.8 \%$ \\
\hline Drinking, & 13 & 8 & 21 \\
\cline { 2 - 4 } $\begin{array}{l}\text { eating } \\
\text { and } \\
\text { smoking }\end{array}$ & $5.9 \%$ & $3.5 \%$ & $4.7 \%$ \\
\hline Shopping & 5 & & \\
\cline { 2 - 4 } & $2.3 \%$ & $3.5 \%$ & $2.9 \%$ \\
\hline Optimism & 68 & 70 & 138 \\
\cline { 2 - 4 } & $30.6 \%$ & $30.8 \%$ & $30.7 \%$ \\
\hline Meditation & 16 & 25 & 41 \\
\cline { 2 - 4 } & $7.2 \%$ & $11.0 \%$ & $9.1 \%$ \\
\hline Routine & 11 & 15 & 26 \\
\cline { 2 - 4 } & $5.0 \%$ & $6.6 \%$ & $5.8 \%$ \\
\hline Do not & 64 & 57 & 121 \\
\cline { 2 - 4 } overcome & $28.8 \%$ & $25.1 \%$ & $26.9 \%$ \\
\hline As
\end{tabular}

As shown in Table 20, among women without children, they mostly cried (20.3\%), or did not overcome the crisis (28.8\%). However, $30.6 \%$ of women reported they were optimistic.

Similarly, among women with children, $19.4 \%$ reported they mostly cry, and $25.1 \%$ report they did not overcome the crisis. However, $30.8 \%$ of women reported they were optimistic.

Research Question 5: Does the patient's age affect how she copes with fertility treatments? To address fifth question, Pearson correlations were conducted in order to assess correlations between age and emotionally coping with their situation. As shown in Table 21, results show that younger women have more support, but they also more negatively affected from fertility treatments in comparison with older women.

Table 21: Correlations between emotionally coping strategies and age

\begin{tabular}{|l|c|}
\hline & Correlation with age \\
\hline Family support & $-.234^{*}$ \\
\hline
\end{tabular}




\begin{tabular}{|l|c|}
\hline Partner support & $-.321^{*}$ \\
\hline Effect of treatment on relationship & $-.281^{*}$ \\
\hline Effect of treatment on routine & $-.315^{*}$ \\
\hline
\end{tabular}

$* \mathrm{p}<.05$

\section{Themes Analysis}

After dividing the interview subjects into categories, the researcher decided to structure a model of five themes as following (see a sketch of one theme as an example further on):

1. Attempts to conceive and the attendant difficulties

2. Response to finding out about the problem

3. Choosing an appropriate solution and beginning treatment

4. Coping with the outcomes of the chosen treatment

5. Being in treatment.

sub-themes:

- The patients' support resources - These constituted six additional sub-themes: Israeli society, the family, the spouse (male/female), friends, work, and the medical system. For each subtheme there were two additional sub-themes-pro or con- meaning whether those support resources in fact support the patient or generate stress.

- Thoughts - The thoughts arising in a patient in the course of a treatment process could be either positive or negative (generating two additional sub-themes). Examples of negative thoughts might be that the anticipated pregnancy would never happen, or why for others it happens so easily. An example of positive thinking and faith: It will happen.

- Feelings - Feelings aroused in a patient following the treatment process could be positive or negative (generating two additional sub-themes).

Examples of negative feelings: frustration, despair, anger. Example of a positive feeling: hope.

- Actions - What a patient did in each situation: deciding to proceed to more advanced treatments, staying with the current treatment, or taking a break from treatment. The two additional sub-themes were advance or withdraw. 


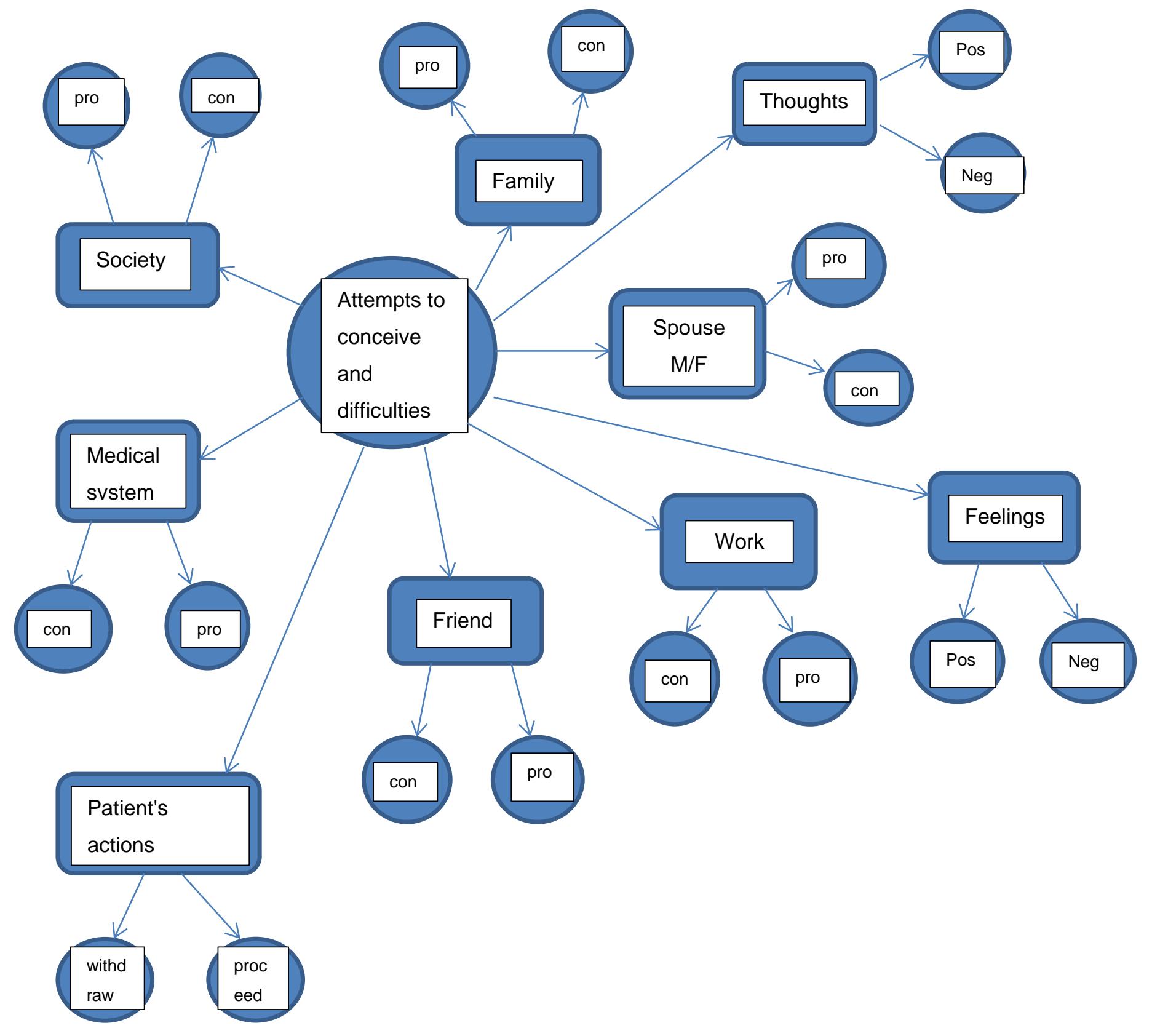

Figure 11: Theme model

\section{Attempts to conceive and the attendant difficulties}

Most people aspire at becoming parents one day, and believe that when they decide to conceive proactively, pregnancy will arrive within a month or two. Some find to their disappointment that in many cases, conceiving is not an easy and fast process and occasionally it takes a while until fertilization is successful. Some try for months or years, and the anticipated pregnancy does not arrive. This process is often accompanied by great deal of stress, especially when it does not happen immediately. 
Interviewees told that they did not think they would have a problem conceiving and the moment they want to conceive it will happen easily, following are the quotes:

L. Z. - "You think that nowadays you conceive easily and you are even told about contraceptive methods and within a second you can get pregnant and suddenly you find out that it is not really the case, we tried for about a year and it didn't happen so we decided to go to a gynecologist".

L. A. - "Truth to be told, I thought that this time I will also conceive with same ease as in last time but unfortunately it didn't happen, it's been eighteen months I've been trying to conceive which is pretty intensive and it would not happen".

H. C. - "I stopped taking contraceptive pills with the purpose of conceiving, I've been told it can cause irregularity in the monthly period for at least several months, but after a few months I did not get the period, I hoped that I was pregnant, but the pregnancy test was negative, so I went to a gynecologist for inquiry".

H. T. - "We didn't think there would be a problem, indeed I am considered to be a fertile woman so there is no reason not to".

S. B. - "I thought I will conceive easily and in one shot and that's it... and it wasn't like that".

C. L. - "In the previous times we had no problem to conceive, I can't say I was conceived immediately, it took time, but we did not get to treatments, and now when I wanted to, I saw it was harder and harder and it was not working. It doesn't work out and it has already been five years on and off that I keep trying".

G. Y. - "Right after we got married, we did not try immediately to bring children since I have studied, and it was important for me to complete the degree. We thought that when the moment comes, and we would want it will happen easily, but it wasn't like that and after almost a year of trying when we realized it doesn't work, we scheduled a doctor's appointment".

N. S. - "You say, why me, why doesn't it work out for such a long time?"

R. Z. - "When it didn't succeed, I was broken since I was sure I will conceive and be pregnant. I really felt this time it will happen, since I don't have a problem".

C. G. - "I was sure it will happen in the first IVF treatment, since there is nothing wrong with me". 
P. G. - "I was sure I will conceive immediately, since I have never tried, and I have no problem".

H. A. - "I thought the process will be fast and I will succeed in conceiving after one treatment, however, much to my surprise, it didn't happen".

\subsection{Support systems of the patient}

The support system of a patient which are: the society, the family, the spouse, friends, workplace and the medical system can be a source of support for a patient, however, can as well serve as a source of pressure and occasionally lack of support for her. Support / lack of support from these factors depends as well on whether a patient does or does not share these factors.

\subsubsection{Society in Israel}

Israeli society is conservative regarding family institution and childbirth. Fertility and birth occupy a key position and in Jewish perception the commandment to procreate is important and fertility is perceived as a blessing. The society assigns great value to family and children and it is founded upon pro-naturalistic ideology, meaning, ideology considering personal and social advantages in giving birth to children and raising them, and as a result adopts positions, beliefs and ways of operating that encourage childbirth. Combination between the social expectation for more than one child with awareness of biological clock, increase mental stress that women anyway are in.

The close environment, being such, levels pressure on the women on this subject whether by questions or by direct statements and the women cope with prying questions and occasionally bordering harassment. Some women are embarrassed and even hurt and they not always know how to react, and some try to justify the condition.

Most interviewees told that they experienced lack of support on part of the society, as they tell:

C. L. - "Sometimes they ask, don't you want another one? And this is hard for me".

G. Y. - "Anyone asking me, how long have you been married, I would lie and say, almost for a year, because I know the next question is how many children do you have? People just don't manage to cope with it". 
H. C. - "I have no patience for people who don't know and who are not sensitive who ask, and what about children, aren't you interested? Usually I shut up and don't know what to answer."

L. A. - "Sometimes I come across questions of why only one child? What about additional brother?"

N. S. - "It was hard for me to hear their remarks of, why aren't you married yet, and what joy children are".

K. G. - "I would receive remarks: what is going on? Is there someone? You know you don't have a lot of time if you want to have some children".

H. A. - "It is not easy to be in this situation especially when I am asked all the time: what about marriage and children?"

H. T. - "People who don't know me and see my wedding ring, ask: don't you want children? This is quite painful".

Whereas three patients felt the environment is actually a source of support for them:

L. Z. - "After the miscarriage they tell you that you need to wait for a year, it can be stress, it can be that you are stressed, and somehow it encouraged me".

R. Z. - "A friend at work took me for a talk and made it clear to me that family and children are forever while career ends. She shook me up a little and out of caring".

P. G. - "I had a fiend writhing right in front of me searching for a way without hurting me to offer me to go for single parenthood, it was clear to me that it was out of care".

\subsubsection{The family}

Close family as well as surrounding society puts psychological and mental pressure on women to give birth to children. The close family ties and expectation of both the mother on a man's side a woman's side, adds substantial pressure upon the couple. Additionally, women cope with tactless statements and prying by the close family. Some women consider this as invasion to their privacy, feel bad and keep quiet.

Most interviewees said that family was a source of stress for them, as they describe:

L. Z. - "I've got pregnant two months after the wedding and truthfully I wasn't ready for that full heartedly, I was simply under family pressure". 
G. Y. - "Our family didn't stop talking about how children are joy and why aren't we pregnant and we always tried to gently push them away and apologize that this is not the right time".

L. A. - "I sometimes would get remarks: your son is alone, aren't you going to give him a brother? He is bored alone".

N. S. - "The family started to wonder and give me hints regarding marriage and children".

R. Z. - "Once in a while my mother throws at me: what is up? You know you are not getting any younger as the years go by".

K. G. - "I wanted it to be from a spouse and the family also started to put pressure regarding the subject".

H. A. - "I find it difficult to see the faces or half-questions each time from the family".

S. B. - "I was hoping that with that the family will also not nag too much".

R. G. - "I am not in close contact with the family and they are not supporting, it's a little twinge in the heart".

Whereas three interviewees felt that family is a source of support for them, as they described:

K. L. - "Generally, the family knew we want another child, but have not put pressure on the subject and also have not been asking too much".

H. C. - "In the beginning the family didn't badger too much since they knew I need to graduate first".

P. G. - "The family encouraged me to wait for the right guy, that seemed logical to me at the time".

\subsubsection{The spouse}

Couples encountering difficulties in conceiving naturally encounter feelings of failure and anxiety should pregnancy linger; wonderings and questions begin arising as to why pregnancy delays and try and understand why it happens specifically to us. The revelation regarding it that not always happens immediately puts the couple system into a complex situation, the expectations, and pressures around the will to conceive might adversely affect intimacy.

Two female interviewees married to a spouse told that the spouse was a source of support for them, as told by them:

G. Y. - "After we felt it is time, we stated trying". 
L. A. - "We wanted another child, because we saw that our son is bored alone".

Opposite to them, three female interviewees with a spouse told that the spouse was not a source of support for them, as they describe:

L. Z. - "The spouse didn't want to have children right after the wedding, he wanted to wait a little".

K. L. - "Although my husband wanted another child, he was not as eager as me".

H. C. - "A year after the wedding I decided I want a child, my husband had mixed emotions". In interviewees with a female spouse, 4 of them had support from the spouse, as they said:

H. T. - "I wanted to experience motherhood and my spouse encouraged me".

R. G. - "A will arose in me to give birth to a child that would establish our intimacy and the spouse supported my decision".

N. A. - "We decided together that I will be the first to get pregnant".

L. H. - "We decided we want to conceive at the same time".

Whereas one interviewee with a female spouse described that at the beginning she did not receive support from the spouse as she describes:

S. B. - "After two years of intimacy we wanted to have a child, we had differences of opinions at the beginning regarding who will conceive first, since both of us wanted to be a mother".

\subsubsection{Friends}

Significant friendships have great measure of honesty and inclusion, and good friends help to be happy. The presence of some is positive, causes to study, develop and grow, while the presence of others sucks from one all positive energy.

As we grow apart from our friends in times of crisis, we send them a message we do not want to be in contact with them. Maintaining a relationship is a work requiring time and resources, however, it is most rewarding. Occasionally, best friends say things that might be interpreted as prying and hurting despite their good intention.

Most interviewees said that they felt lack of support on part of their best friends, as they tell: 
L. Z. - "We have many friends some have even married after we did, that we were in good relations with them and they already have children, it annoys me when they tell me: your life is good you sleep all night, be thankful".

K. L. - "In the beginning we had a lot of friends and slowly connection with them loosened, once in a while we see each other and then when they see the stomach popping out, they immediately say I see there is something".

G. Y. - "I have some friends from work, but I don't share with them, when they asked me at the beginning: do you have children? I answered rudely that is was not their business".

H. C. - "I told a good friend from work that we try and don't succeed, she said - luckily we don't have this problem, it happened for me with ease".

N. S. - "I have some friends who are my customers and when they speak about their children, they tell me - you will not understand because you have no children yet".

K. G. - " I have two good friends that have children and sometimes when we meet they tell me with excitement about their children and ask me - doesn't it make you want?"

P. G. - "My aunt is my best friend, however occasionally she allows herself to enter my private life and nag me about wedding and children".

H. A. - "I have some good childhood friends that remind me each time - despite your brilliant career you also need to think about starting a family".

R. G. - "We don't have so many friends".

Whereas three patients said that they received support from their friends, as they describe:

L. A. - "When I told my childhood friend that we try without success, she offered me to purchase from the pharmacy an ovulation kit".

R. Z. - "My childhood friend has gone with me through the hesitation whether or not to start the process".

H. T. - "We have mutual friends both of us that have gone through the process and are very supportive towards us".

\subsubsection{Workplace}

Work nowadays is an important component in people's life and their wellbeing. Everyone is interested in finding a job in a company that offers good and comfortable work environment, 
and naturally all employees look for a challenging and interesting work, with good people around them, professional and calm bosses, and adequate pay conditions. However, while planning the expansion of a family, brain and heart are troubled with the attempt to conceive and the mental state of an employee is not always lifted, which occasionally can cause problems in functioning at work.

Most interviewees except for one have regular work for several years, in stage of attempts to conceive it was irrelevant to include the workplace, therefore, in this stage, there is no reference to this subject.

\subsubsection{The medical system}

In this stage of attempts to conceive, there is still no application to the medical system. Two interviewees said that after few months of trying, they turn to medical help, but the doctor claimed it was not the time yet and that they should keep trying naturally.

L. Z. - "After 6 months of attempts I applied to a gynecologist and he said it was too early to begin an inquiry and recommended to try for another six months and if I do not get pregnant to come back to him".

H. C. - "I consulted my gynecologist who told me that I might not be calculating ovulation correctly".

\subsection{Thoughts}

When attempts to conceive fail and pregnancy does not occur, first doubts begin to pop up and negative thoughts run through the head that possibly something is not right and then wonderings begin of why doesn't it happen?

Most interviewees said that negative thoughts have arisen.

L. Z. - "Why doesn't the pregnancy, not coming easily? Maybe I make a big deal out of it and I should wait?"

K. L. - "It bothered me that it's not working, but we were too busy to delve into it so I cast it aside".

G. Y. - "When is it time to get stressed? The head starts running in all directions, what's wrong?" 
H. C. - "In the first year we were newlywed and there was no room for disappointment, we lived, we had fun and travelled but after a year of attempts thoughts have started, why just not me?

L. A. - "Expect for following month and also then pregnancy does not arrive and also in following month... movies start running in my head that maybe something is wrong".

N. S. - "Why is it that nothing in my life goes easily? How did I come to that situation?"

R. Z. - "Sometimes thoughts arise in me that create dark future that maybe I will not have children, why did I wait for so long?"

K. G. - "Fear it will not happen, fear of a long waiting period".

R. G. - "The natural, simple and obvious thing become unexpected, uncontrolled and unnatural".

H. T. - "Fear that the family will not accept our new familial situation".

L. H. - "Suddenly it is stressful, we are going to make a huge and bold step".

Whereas three interviewees had specifically positive thoughts:

H. A. - "Thoughts arose in me, whether to wait for an intimacy partner or to fulfil my desire for motherhood?"

S. B. - "Thought of going to a new path that will bring about the expected pregnancy and luckily there is medical-scientific possibility to help".

R. G. - "Hoping that the coming baby will manage softening our families".

\subsection{Feelings}

Attempts to conceive begin with excitement and expectation, after several months without success the worrying stage arrives, over time, worries become a sort of stress and lack of certainty causing the couples to apply for examination.

Most interviewees reported negative feelings following failed attempts to conceive:

L. Z. - "I was in shock, I thought it was so natural, there were many wars, to say it in simple words, many blows".

G. Y. - "I thought it would come the moment I would want it, I had a disappointment and a sense of failure". 
H. C. - "Negative feelings arise in full strength especially several months of expectation and disappointment".

L. A. - "I felt constant frustration and pressure, when will I be pregnant already?"

R. Z. - "I felt fear from the unknown, is this the right time?"

K. G. - "I felt uncertainty, is this really what I want?"

P. G. - "I felt sadness regarding current situation".

H. A. - "I had fears, is this going to prevent me from realizing myself?"

S. B. - "With the excitement there was a slight fear of what will happen?"

H. T. - "I felt a little stressed to make this move".

Whereas two interviewees said that they had rather positive feelings:

K. L. - "Despite everything, I tried to be optimistic and calm".

N. S. - "I felt excitement with starting the process".

\subsection{Patient's actions}

Failure in recurring attempts to conceive put a patient in front of two options: to advance or to withdraw.

Most patients decided not to wait and apply to a gynecologist / fertility doctor for inquiry, as they describe:

L. Z. - "We decided to go to a gynecologist for inquiry".

G. Y. - "I decided to go to a gynecologist because I had a feeling that something is wrong".

H. C. - "After more than a year that we tried, we went to a gynecologist".

N. S. - "I went to a gynecologist to start the process".

K. G. - "I pondered a lot but eventually I scheduled an appointment with a fertility doctor".

P. G. - "I decided to go to a gynecologist to hear about the process".

S. B. - "We scheduled an appointment to start the process in a hospital".

H. T. - "We scheduled an appointment with a gynecologist to start the process".

L. H. - "We looked for a good professional doctor to go to".

Whereas five interviewees decided not to go forward at this stage: 
K. L. - "I wanted to go to a gynecologist for inquiry however, my husband asked that we try naturally for few more months".

L. A. - "We wanted to go to a gynecologist but decided to continue trying alone, if in a few months does not happen we will go".

R. Z. - "I scheduled an appointment with a gynecologist and in the last minute I had cold feet and didn't arrive to my appointment".

H. A. - "I scheduled few times an appointment with a gynecologist but cancelled every time".

R. G. - "We scheduled an appointment with a private doctor and a moment before we went to him, we cancelled the appointment because we heard negative critiques about him".

In summary: the process of attempts to conceive begins with great expectation, suspicion that something is wrong awakens as early as after few months of failed attempts to conceive and the expectation turns into disappointment and frustration.

\section{Response to finding out about the problem}

When more than a year goes by in in attempts to conceive without success, one applies to a gynecologist for examination. Mostly an assessment is made of fertility state of a woman and her spouse (comprehensive blood tests, PAP, uterus X-ray, sperm check). The procedure opens with examining a woman's condition and should a problem be found with her she will as well be the last and only one to be examined. A man will be reached only should checking the woman ends up with nothing.

Knowing that there is a disruption and that there is a fertility problem results in severe reactions of shock, morning, denial, anger, repression or an emotional outburst, sadness, and depression. Most interviewees told that during the examination it was revealed that for some the problem was in them, for some in the spouse and for some no problem was found at all and the inquiry continued. As put in their words:

L. Z. - "I remember after taking all the tests, I received a phone call from the doctor asking us to come to a meeting and then he told me he understands why I cannot conceive, it is due to my husband having a problem, his sperm is not good."

K. L. - "I decided to go to the doctor for examination because I saw that there is a problem and it takes time, we took all the possible tests but no specific problem was found". 
G. Y. - "I had polycystic ovaries, the sonar showed water in the left ovary so the doctor sent me to uterus X-ray, where they found both my tubal obstruction, tried tuboplasty, with one it was successful and the other remained blocked."

H. C. - "The sonar I took revealed polycystic ovaries and the sperm test my husband took was not good also."

L. A. - "I took a lot of blood tests and even uterus X-ray and everything was in order, my husband's sperm test was also good."

N. S. - "The sonar revealed water in the ovary, so I did a uterus X-ray and then laparoscopy and my ovary was taken out".

R. Z. - "The doctor referred me to all sorts of tests, I was diagnosed with endometriosis".

K. G. - "In the blood tests I took I had a slightly high FSH."

H. A. - "My AMH test was extremely low."

Opposite to them, four interviewees told that they knew they will experience difficulty in conceiving and that they have mentally prepared themselves to the process. As they describe:

P. G. - "Truthfully, I mentally prepared myself it will take years for me to become a mother if I will ever be a mother at all."

S. B. - "I knew I would have to undergo treatments to become a mother."

L. H. - "I knew it will not happen naturally."

N. A. - "I've taken into consideration it can happen."

\subsection{Support systems}

Occasionally a concern arises from a discussion regarding the subject with family members, friends, the workplace, or professionals. All these intensify should preliminary tests reveal a fertility problem in one or both spouses and occasionally it is not even possible to trace the source of the problem.

\subsubsection{The Israeli society}

In country where everybody asks continuously - "when will you have children?" or "why are you not pregnant?" and actually instead for the subject of childbirth be intimate it became a public interest in which everyone has the right to express their opinion and give advice even though they are not required. 
Most interviewees told that they had a difficulty due to remarks made by the environment, as they described:

L. Z. - "Everyone around me got pregnant and gave birth and only I wait and when they wish me "I wish you the same soon" it makes me cry."

G. Y. - "I don't like to be pitied, I learned to answer with sarcasm that it will come when it comes."

H. C. - "Someone told me not long ago that's it you finished partying, you flew, you had a good time, you had fun, now the time has come to work on children".

L. A. - "I was told I'm lazy that my son does not have a brother yet."

N. S. - "I was asked more than once - couldn't you find someone to marry with? What is wrong with you?"

R. Z. - "I was asked how is it possible that you are not married yet and you still don't have any children?"

K. G. - "I have been asked why did you wait until now? This is not a simple process."

R. G. - "I got a response - maybe it is worthwhile to think about it a little longer?"

N. A. - "It feels the environment looks at us with disapproval".

L. H. - "The close environment disapproves, asks questions, criticizes and judges, try to explain how complexed the step is".

Whereas, three interviewees actually gained support from the environment, as they tell:

K. L. - "I was told you already have children; you don't need to freak out."

P. G. - "I was told go for it, later it will be too late, and you will be sorry."

H. A. - "Those who know that I intend to get pregnant say - wow, how brave you are."

\subsubsection{The family}

Knowing that there is a difficulty in conceiving whether the problem is clear or not, puts the family in a situation that they really want to help however they do not know how to do it and what to say. They occasionally give advice, criticize, and make concerned remarks, they think everything is allowed and it is hard to ignore them, some women become offended by that, as they describe: 
G. Y. - "Most annoying is that someone in the family gives birth, and then all the family feels sorry for me and wishes the same for me soon."

H. C. - "The family suspects there is a problem, every once in a while they ask well, what is going on? Any news?"

N. S. - "It bothers me that I am compared with neighbors that got married and already have children."

R. Z. - "My mother hopes I will get married and bear children like everybody else and I feel I disappoint her."

K. G. - "There was objection on the part of my mother in the beginning, she was not for it."

H. A. - "My sister thought I am making a mistake and stopped talking to me."

S. B. - "At first the family didn't want to get involved and that stings in the heart."

H. T. - "The family knew, however not everyone supported."

R. G. - "The family opposed to all of it."

Whereas, five interviewees described that they have received support from the family:

L. Z. - "My mother said - don't worry, all is in due time, that calmed me down a little."

K. L. - "My mother told me look at what you have and not what you don't have."

L. A. - "The family sees that for a long time I'm only with one child, they realize there might be a problem, but they don't ask".

P. G. - "It took her some time, but my mother was convinced that otherwise I will remain with no children, so she decided to help me in this journey."

L. H. - "The truth is that the family was very surprised, however my sister supported us."

\subsubsection{The spouse}

Many a woman decides to compartmentalize the spouse from the process in order to "protect them", however, by doing so they increase the distance between them and the sensation of partnership in the process as they make them redundant. This experience awakens in them a sensation of embarrassment and helplessness and makes them put all their effort into something else. Men are naturally less overt regarding their feelings; they might struggle with them, so they do not have to deal with the difficulty and express it.

As three interviewees who have a male spouse describe: 
L. Z. - "As soon as we discovered the problem is in him, he was embarrassed, it took him time to enter the process."

K. L. - "It took a few months until my husband agreed to take the sperm test, he took it hard".

G. Y. - "I started the inquiry alone, my husband preferred in this stage to wait with his test."

Whereas two interviewees with a male spouse felt supported by him:

H. C. - "My husband really wanted me to conceive naturally, he was upset for both of us."

L. A. - "My husband tried to cheer me up that it will come out all right and that it will happen eventually".

Despite the fact that interviewees with female spouse know they will need treatments in order to conceive, for them it also appears a scary, stressful and shaking journey.

Four of them had support from the spouse as they describe:

S. B. - "My spouse promised to be by my side throughout the whole journey."

H. T. - "I was a little afraid of the process however my spouse encouraged me to trust her and that it will be all right."

R. G. - "I received enormous support from my spouse to begin the journey towards motherhood".

L. H. - "We were excited to begin the journey in parallel while supporting one another".

Whereas N. A. told that in the beginning she has not received from her spouse the support she expected to get:

"My spouse suddenly got scared from the process and said don't you think it is too early? Maybe we should wait a little longer?"

\subsubsection{Friends}

On many occasions friends have no knowledge of what to say and how to help, at times they have difficulty to express empathy, make tactless remarks and as well offend at times. Some, however, not understanding, try in their way to be empathetic and mainly supportive... as the interviewees tell:

L. Z. - "I told a very good friend of mine and she said oh, poor you... luckily we don't have this problem, with me it happened easily".

G. Y. - "A friend asked me - a third child, for what? And what if it is a girl again?" 
H. C. - "My friend told me she was conceived by mistake and that now it is not the right time for her".

N. S. - "My friends tell me about their children and that hurts".

K. G. - "A good friend asked me - shouldn't you wait a little longer?"

P. G. - "One of my friends thought it was a mistake".

S. B. - "My childhood friend frowned at me when I told her I'm starting a process".

Whereas five interviewees told that they received support from their friends:

K. L. - "A good friend said you are not the only people who have a problem, there are many solutions today".

L. A. - "A friend of mine encouraged me and said - don't worry, all in due time".

R. Z. - "A good friend offered to accompany me during the entire process".

H. A. - "Some of my friends supported my decision".

L. H. - "Despite saying it is difficult, our friends supported our decision to conceive in parallel".

\subsubsection{Work}

In the stage of infertility inquiries, patients are required to undergo tests that occasionally might collide with working hours. Most patients do not tell about the problem in this stage at work and therefore, in order not to miss work hours / be absent from work, patients try to route the tests so it does not harm working routine and sometimes justify why they are late, as they describe:

L. Z. - "There were days that I came late to work, I would say didn't feel well".

K. L. - "I didn't tell at work because I heard the environmental responses of my subordinates undergoing treatments and I did not want to be in that place, so I took the tests privately".

G. Y. - "I tried not to miss working days during the tests and inquiries, sometimes it was not easy".

H. C. - "I made an effort routing the tests to my free days or during evening hours in order for them not to notice".

K. G. - "Sometimes the tests fell on working days, so I took sick leave days and the boss didn't like it". 
P. G. - "I decided not to tell at the moment therefore I had to juggle with the tests so it does not harm my work".

H. T. - "Occasionally I would come to work late and had to make excuses...".

N. A. - "I didn't tell at work, because I was afraid that they will not understand".

L. H. - "It was difficult to maneuver between work and the tests without them noticing... I had to find excuses".

Whereas six patients felt they received support from the working place:

L. A. - "In the beginning at work they understood when I was occasionally late, I tried it does not happen often".

N. S. - "I have my own business, so I had flexibility with the tests at work".

R. Z. - "I told the manager I was starting the treatments, at the beginning she was supportive and said she will do everything in her power to help me."

H. A. - "I told my partner I was starting with the process, he was pretty much supportive".

S. B. - "It is an advantage I am a business owner, I did the tests when it was convenient for me".

R. G. - "In the beginning we managed to time the tests with work".

\subsubsection{The medical system}

Frequently patients apply to private doctors who are impatient regarding giving answers to questions, scarce with explanations and give little information. Mostly patients are restrained regarding that due to the simple reason that this is a good doctor with excellent successes. On the other hand, there are doctors giving a lot of information that makes them confused and they feel lost in the medical system. Occasionally doctors give tests without explaining the reason and there are even cases in which there is no referral to test from some reason and in retrospect it appears that actually a preliminary and more comprehensive test would have indicated a problem that could have been discovered earlier.

As they describe the lack of support:

L. Z. - "The doctor was very direct and said indifferently that in light of the sperm problem we will not succeed to conceive naturally, at that moment I felt like a 500 tons' rock fell on me". 
K. L. - "We left the doctor confused with a lot of forms and tests to take, we didn't know how to process all that information".

L. A. - "I cope with overweight and used to the lovely "advice" of all doctors in that subject".

R. Z. - "The doctor told me with a severe face that in my condition I will need IVF treatments and that I shouldn't waist time".

K. G. - "The doctor was a little impatient towards me and didn't let me ask all the questions I wanted to, he said go to the nurse, she will explain to you".

P. G. - "I came across many obstacles from the medical system, be it improper answers to a test I took, making appointments to tests and their availability and the exhausting bureaucracy of the system".

H. T. - "We felt that the doctor doesn't really care, and the responses are robotic and automatic without any sentiment, although he was highly recommended".

N. A. - "The doctor didn't refer us to genetic testing, said that in my condition it is irrelevant".

L. H. - "The doctor didn't bother sending me to PAP test, claiming I don't need it since I am not in high risk group, the medical team was also a little sceptic about us and we felt they found it difficult to support us".

Whereas four patients felt they received support from the system:

G. Y. - "The doctor was considerate and agreed to postpone my husband's sperm test for now".

N. S. - "I went to the nurse with all the forms and she received me with a smile, sat with me patiently and explained the entire process".

H. A. - "After I left the doctor with lots of forms the nurse gave me a sense of confidence that she will accompany me along that journey, and that calmed me down".

S. B. - "Although the doctor didn't say much, on the other hand we felt the clinic staff is very inclusive and willing to help".

\subsection{Thoughts}

When the problem is already known, or the problem cannot be figured out and there is already a "headline" of infertility, there is a fear of the unknown, uncertainty and an expectation that the problem will be resolved. Not always it is known what is coming and therefore occasionally as a result of that negative thoughts arise, as they describe: 
L. Z. - "I realized that from this point it is not going to be as easy and natural as I have dreamt. I couldn't stop asking myself: why me of all people? How is it possible that I planned my life for me, and it just doesn't go?"

K. L. - "At that night many thoughts run through my head - it was unimaginable there could be problems, problems? It doesn't really happen to healthy people like us, problems are for people with problems, how is it possible?"

G. Y. - "Sometimes there were thoughts of will we survive the difficulties and succeed in having a family?"

N. S. - "Ohh... the thoughts, the worries, the fears. The main question did I make the right choice?"

K. G. - "I would think what did I do wrong, why don't I deserve the whole package (a spouse and a child), I don't want to go through the process alone and raise a child alone, it doesn't seem fair to me towards the child that he will not have a father."

P. G. - "I had thoughts that really bothered me as early as at the stage of misgivings, such as life without a spouse, without a father figure, how to do it alone (logistically and financially), it was stressful".

H. A. - "I have a strong will to bear a child, but this is not the way I have imagined myself starting a family, how come treatments now?"

N. A. - "We had thoughts regarding the procedure and all the people and procedures involved in it".

R. G. - "We had concerns regarding how the environment will accept that".

Whereas five patients had positive thoughts, as they describe:

H. C. - "Despite being a little afraid, I had thoughts that despite everything, it will happen soon".

L. A. - "In the beginning I was thinking, well, if there is no problem it means there is still a chance it will happen naturally?"

R. Z. - "I tried to tell myself that it doesn't matter, and the main thing is being a mother, therefore I thought about the possibility of shared parenting, so that the child has a father".

H. T. - "I had thoughts about a new way, thoughts of hope, thoughts of success and thoughts about a family". 
L. H. - "We trusted the medical system giving us the opportunity to become a mother".

\subsection{Emotions}

A condition of near crying, lack of enjoyment from things usually enjoyable, sleeping or eating problems, and nervousness due to the fertility problem and the inability to conceive, is a natural reaction. Infertility causes intense feelings of envy and anger towards the fertile world, including friends, neighbors, brothers and sisters. Coping with such negative and extreme feelings is difficult. Some women suffering from infertility feel awful about themselves as a result of those emotions feelings and their presence brings about feelings of guilt and shame. As most of them describe:

L. Z. - "I felt my world became dark, I called my mother and told her the verdict while I was sobbing".

K. L. - "Mainly I felt sadness, as there is no apparent problem".

G. Y. - "At this stage every friend, neighbor, aunt got pregnant and I didn't. On one hand I tried to be happy for them and on the other hand it hurt and I found myself jealous of them and later I felt bad about myself for feeling this way".

L. A. - "It was a surprise we have not expected, we were disappointed".

R. Z. - "I didn't sleep all night; I could not stop crying all day. I took the process and the distresses to a personal place, and therefore I felt extremely lonely".

K. G. - "I felt I was going to the sperm donation really heavyheartedly, I waited for someone to rescue me from that at the last moment, like in the movies".

P. G. - "I felt bitter, why can't I easily realize the dream I had in life? I was quietly wiping a tear without anyone noticing".

S. B. - "The fact that I am healthy yet needing to go through medical procedures was difficult for me".

Whereas four interviewees had positive feelings, as they describe:

H. C. - "We were in shock at the beginning, but the desire for a child has overcome it all".

N. S. - "In the beginning I had mixed feelings, I felt a little confused, a little overwhelmed, but above all very excited".

H. T. - "Knowing I am going to be a mother and build my family really excited me". 
R. G. - "I was tremendously excited, and I had hope it will happen fast".

\subsection{A patient's actions}

At this stage, a patient has an option to begin fertility treatments or go for a second opinion from another doctor and there are those who are even interested in waiting a little longer and not begin with the treatments. Occasionally fear and stress cause them to back down and at times they are interested in trying naturally a little longer, maybe it will happen...

Interviewees who decided to advance describe:

K. L. - "I decided to start with the treatments despite the fact that my husband opposed and suggested we wait".

G. Y. - "We decided to begin with the process and made a doctor's appointment".

H. C. - "After thinking about it together we decided to make a doctor's appointment to start with the process".

N. S. - "I decided to have courage and begin the journey".

P. G. - "I decided to go for it till the end".

H. A. - "Despite the concerns I decided to go to the treatments and begin".

S. B. - "We went for it, we knew we were doing the right thing".

H. T. - "We decided to begin treatment in order to make our dream of becoming a family come true".

R. G. - 'We went back to the doctor to start the treatments".

L. H. - "We went for it wholeheartedly".

Whereas five patients decided to withdraw at this stage, as they tell:

L. Z. - "We decided to go to a private doctor to get his opinion before we enter the treatments".

L. A. - "At this stage we decided to try a little longer independently, and if it does not work then we will go back to the doctor".

R. Z. - "I got cold feet from the process and also from the criticisms I got from the family".

K. G. - "I went for a second opinion of another doctor before entering the process".

N. A. - "I got a little scared of it all, I wanted to think about it a little longer". 
In summary, from the moment the fertility problem was revealed, the fear and stress that patients feel increase, along with it there are excitement and expectation the proposed treatment will bring about the awaited result quickly.

\section{Choosing an appropriate solution and beginning treatment}

Fertility treatments are given according to the problem revealed following various tests. In cases the problem is not clear the treatment is done by trial and error, meaning rendering different treatments in order to receive the desired result. Regardless the source of the problem, in any case a woman is the one who needs to undergo the invasive medical procedures. This is the beginning of a journey which end is indefinite.

L. Z. - "In the beginning the doctor suggested treatment with insemination, I did 3 such cycles and later another 3 with hormones".

K. L. - "The doctor suggested we start treatment with Ikaclomin pills and that we will also do an insemination".

G. Y. - "We started doing inseminations with Ikaclomin, I have undergone 3 such cycles".

H. C. - "We started treatment with Ikaclomin and insemination, I have gone through 2 such cycles".

L. A. - "The doctor proposed to start with hormone injections and inseminations, however we preferred in the beginning not to do insemination, but rather it will be more natural".

N. S. - "In the beginning the doctor proposed hormones with inseminations, I have gone through 2 such cycles".

R. Z. - "I started with a treatment of Gonal and insemination and after few months I had another such treatment".

K. G. - "Despite the fact that the doctor proposed I start with medication treatment with Gonal, I wanted to do a natural cycle and insemination. I did one like this and later 2 cycles with Gonal".

P. G. - "In the beginning I went through natural cycles and inseminations, in total 2 of these".

H. A. - "I started a treatment cycle of hormones and insemination". 
S. B. - "We decided to do one cycle of ovulation follow-up and insemination and later I advanced to treatment with Ikaclomin and insemination".

H. T. - "In the beginning I have gone through 2 natural cycles and inseminations".

N. A. - "In the beginning I did 2 treatment cycles without hormones and inseminations and later I advanced to treatment with Ikaclomin, in total 3 treatments".

L. H. - "In the beginning I have gone through natural cycle and insemination, 3 such cycles".

\subsection{Support systems}

In the beginning of the medication treatment a patient has a dilemma whether to choose and tell that she undergoes treatments to the family/ friends/ near environment/ working place or the spouse regarding the difficulties she goes through, or wait until the treatments are a success. Does it help talking or whether it is better to hide and the position of the medical system towards her.

\subsubsection{Society in Israel}

There is social expectation that everyone becomes a parent at one point or another, encouragement to expand the family at almost any cost. There is an unequivocal encouragement, direction, and push towards a course of fertility treatments. There is big public investment in order to guarantee for everyone the right to become a parent. There is a social message that a woman should undergo treatments, even if agonizing, if you will have a child.

Most interviewees gained lack of support and pressure from the society as they describe:

L. Z. - "As the time goes by and they see that I don't have children they keep bothering with questions why can't you get pregnant, so what is your problem if I may ask?"

K. L. - "I was asked, why are you anxious regarding having another child?"

G. Y. - "When I was asked regarding the subject and I hesitated in answering I was told all right, at least do you undergo treatments?"

L. A. - "My son was told do you want brothers? Of course you do, tell your father and mother to get you brothers."

R. Z. - "I came across environmental responses of - wow... this must be hard, and all the more so alone".

K. G. - "I was told don't think children are easy, what do you need it for? Accept your destiny". 
H. A. - "I was told at your age there is a slight chance the child will be healthy".

S. B. - "Around us there was amazement, questions were asked and mainly we were judged regarding the process we go through".

R. G. - "Mainly we were criticized, and attempts made to explained to us how much the step is complex and complicated and maybe we should think about it a little longer".

Whereas three interviewees told they received support from the society, as they describe:

H. C. - "When they realized I still have no children they said - well, presently there are many innovative treatments, don't you worry".

N. S. - "Some people told me don't give up on motherhood, it's worth it".

P. G. - "I was told why don't you do that? Why don't you try?"

\subsubsection{The family}

Women undergoing treatment find it difficult to have remarks from the family, they feel it causes them stress although it stems from good intentions and a desire to help. When the people around are being involved in the treatment process, they begin to defend the patient with a bond of silence (whispers or concealing regarding someone pregnant) out of a consideration that it would be less difficult.

Most interviewees received support from the family, as they describe:

L. Z. - "The family started asking until we told them and then the questions started, well did the results arrived? Did you go to the tests? Go to such and such Rabbi..."

K. L. - "My mother asked so what is the problem, did you ask? What did the doctor say? Oh, what does he know anyway".

H. C. - "They told me you are stressed, as soon as you relax and stop thinking about it, it will come, it's just in the head".

L. A. - "We told the family we are going through treatments, they said they understood it by themselves and didn't want to pry".

R. Z. - "My sister was glad I had finally started on the process and wanted to be involved in it."

H. A. - "despite the difficulty my mother supported me during the treatments."

P. G. - "My cousin supported me and told me, you are the one to be left later with no children and you will be the one to pay the price, nobody else." 
K. G. - "In the beginning my mother tried to discourage me from the process, however when she realized I am determined she offered to help me financially with the treatments."

L. H. - "I told my sister we are going through treatments and she promised to support us in everything".

Whereas three interviewees were not supported by the family as they describe:

G. Y. - "I didn't tell the family because they would take it hard with tears and dramatization and I don't want it eventually I will have to support them instead of them supporting me."

N. S. - "Friday night same blessings and same comparisons to the neighbors who got married, it's not easy".

N. A. - "My mother knows we started the treatments and told me, I'm not sure you are doing the right thing".

\subsubsection{Spouse}

Fertility treatments make the natural process of conceiving saturated with medical interventions, tensions, and uncertainty. The natural, intimate process between spouses on the way to parenting turns into a medical procedure: technical, cold, exposed, controlled by the medical staff involved in all. Intercourse takes place according to instructions; numerous invasive medical tests intensify the sensation that the intimate and personal borders are breached.

As four interviewees describe the difficulty and lack of support from the spouse:

L. Z. - "My husband preferred thinking there is no problem, or that if he ignores it the problem will go away. I felt he expects me to support him while I am barely holding up".

K. L. - "Sometimes it is artificial, suddenly there is a specific date a specific day and specific time to do something and then he is such an outsider, it is hard for him because it is artificial."

G. Y. - "He has difficulty with not being spontaneous of the process of conceiving, doing it because it is ovulation day and we should and this is mechanic, with no desire at times".

L. A. - "At first it wasn't easy, it felt like a task that needs to be done according to an order and not like something done with love".

Whereas H. C. had support and she described that - "despite the fact that it was not simple we knew we were doing it because we have a common purpose and we are doing all we can to achieve it". 
Amongst interviewees with a female spouse, although there were no difficulties of that sort, there was a difficulty in the fact that the process of conceiving occasionally damages their intimacy and causes tensions as there is an involvement of personnel in the process and that is involved in continuous unpleasant procedures. As two interviewees describe:

S. B. - "The routine tests accompanying the treatment are unpleasant (vaginal ultrasound), sometimes my spouse said I was exaggerating".

N. A. - "The IUI process was significantly painful and that caused me distress and, in some way, interrupted our life routine".

Whereas three interviewees told that the female spouse supported them despite the difficulty:

R. G. - "I was embarrassed each time to be exposed by different doctors performing the IUI, I was flinching, and the spouse has always been patient".

H. T. - "All these procedures are involved with physical discomfort, what comforted me was my spouse by my side".

L. H. - "I hated the speculum, only the thought of it made me flinch every time, my spouse would calm me".

\subsubsection{Friends}

Friends try to express sensitivity to the situation, some of them give ideas and advises which are not always accepted positively by the patients, and some of them hide pregnancies in order not to harm. Some of the patients gradually try to lessen social gatherings as they feel they are being pushed aside and they already do not have a common discussion subject with the friends, as the interviewees tell:

L. Z. - "My friend told me she was pregnant, I cried with excitement for her and I was particularly hurt that after two hours I got my period in the toilet at work after first insemination while she got pregnant when she just stopped taking pills".

K. L. - "I have a friend who knows, but is not included in the process, when I have good news I will tell her. She will not understand anyway, she already told me once - it's not a disaster, just a negative answer".

L. A. - "My friends know about the process and the most annoying sentence is in a day of negative Beta - never mind, next time". 
H. C. - "Suddenly I realized that many of my friends who have children suddenly were lost out of sight".

N. S. - "I have some clients who are good friends who asked me what do you need it for? So you did not conceive, never mind."

R. Z. - "I told a friend from work I started treatments and her response was - nice, I don't know maybe out of embarrassment".

K. G. - "I felt I really don't have so much to talk about with my friends, they proudly tell about their children and I feel I don't belong".

P. G. - "I had difficulty in social meetings, it required a lot of mental strength from me of showing everything is all right and everything is normal while I know it is not".

R. G. - "In a meeting with friends when everybody talks about their children I feel an outsider".

L. H. - "Less meetings with friends, they are busy with their things and we are with ours".

Whereas four interviewees received support from friends as they describe:

G. Y. - "I told a good friend of mine who knew to support me and keep my secret".

H. A. - "My friend told me, maybe you are too stressed and offered to give me an Acupuncture."

S. B. - "A good friend gave us an advice to be patient for as long as possible because sometimes the process is prolonged".

H. T. - "One of my friends told me - what good will it do if you worry? This time don't expect so you will not be disappointed".

\subsubsection{Work}

Fertility treatments are an extremely stressed time period in which one is to be very concentrated and at the same time it requires lots of peace of mind. The treatments require daily arrival for tests for a few days consecutively in the morning, not easy to combine it with work that starts at early hours of the morning, which causes a patient to arrive late to work and at times miss some working day due to a medical procedure. As they describe:

L. Z. - "I told at work I'm going through treatments, I felt like everyone walks on eggshells and it made me more nervous".

K. L. - "There was a difficulty to combine between the treatments and a demanding work where there is no consideration and understanding, I had a will not to show weakness". 
H. C. - "In the beginning I completely hid the fact that I am going through treatments. I spent many days off in order not to tell".

L. A. - "I have no problem to be absent for treatments, but the employer forces me to work extra hours without payment, I leave work late".

R. Z. - "At work they are aware of the fact I started treatments and when everybody knows they interfere; they think they know what is good for me and this makes damage and eventually the treatment does not succeed".

K. G. - "My position was reduced following the treatments, I had to take additional job due to that".

H. T. - "No doubt the maneuvers between the tests and work harms work and the conduct during work especially when I am assigned more tasks".

N. A. - "I have terrible overload at work, pressing and demanding manager and this is hard because she doesn't accept my being late in the morning".

L. H. - "Days I was absent from work I had to make up for in another day, I felt sometimes powerless and difficulty to concentrate at work".

Whereas five interviewees received support from their working place:

G. Y. - "In the beginning I would look for excuses and tell I didn't feel good and I would take a sick leave, to my surprise they were understanding".

N. S. - "When I started the treatments I managed to maneuver with the work, I would just stay later sometimes".

P. G. - "In the beginning I made up excuses to my being late in the mornings, later I simply told, and I was surprised I was in fact supported".

H. A. - "There were tests I did privately early in the morning or after working hours and it really made it easy for me as far as work is concerned".

R. G. - "In the beginning we managed to maneuver between the tests and procedures and the activities of our business".

\subsubsection{The medical system}

There are cases in which a doctor prescribes a treatment protocol without giving the patient early preparation regarding the essence of the treatment and its possible implications and 
occasionally the patient has a difficulty to digest or contain the situation. Bureaucracy of the Health Maintenance Organizations at times mounts difficulties for patients regarding tests or authorizations which they claim that there is no indication to do. As the interviewees describe:

L. Z. - "In most cases I paid privately also for tests I asked for and I was not authorized to perform in the HMO, and also to go to the doctor, meaning that if it's not private you can wait for four months and privately already in the following week I would see a doctor".

K. L. - "In the beginning I had the impression the doctor would send me straight to treatments without any deep knowledge regarding my fertility condition, he told me try at first some cycles like that and if it will not work than we will consider additional tests or additional options".

H. C. - "The doctor told us: there is a very easy solution to the problem - insemination (IUI) and the chances of success are very high because you are a young couple and in one or two cycles you are pregnant, ah, and you should take these pills without explaining possible side effects, ah, you should also take blood tests and ultrasound ahh, and also buy this injection that before the insemination you should inject in the stomach and you can go to the nurses in the HMO it's not a big deal".

L. A. - "The doctor decided on medical treatment with injections, said it is very user friendly, just attach to the needle, find the right dosage and inject. What are the side effects I asked, he said why would there be any side effects? Everything will be all right".

N. S. - "It was difficult for me that the Hysterosalpingography was hard and they didn't give me antibiotics right away because I was hospitalized a few days later with an inflammation in the uterus and received antibiotics through the vain".

K. G. - "I found the fact that I would have to inject hormones difficult to process and the nurse didn't have the patience to explain to me regarding the process of injecting, therefore the doctor decided I will do the first cycle without hormones".

P. G. - "The insemination process (IUI) was difficult and painful, the doctor had difficulty getting in with the catheter and I screamed with pain, the doctor said why are you screaming, it doesn't hurt."

H. T. - "Sperm bank were good in the beginning, little less afterwards. There was lack of synchronization, partial/wrong information at the critical moments before insemination, when it is already heavy emotional burden". 
R. G. - "In the beginning they forgot to prepare a notice of obligation for release of the dose and the Sperm Bank did not agree releasing the dose."

N. A. - "You cannot say the staff are liberal, some have mental blocks regarding a couple of two women who want to have children, there is already a tremendous mental pressure due to the treatments".

L. H. - "Once they tripped us up with insemination, we had to run about in order to release the sperm dose and then arrive to the clinic for the procedure".

Whereas four interviewees felt they did receive support from the medical system, as they describe:

G. Y. - "After a few failed cycles of insemination, the doctor sent me to do Hysterosalpingography, there was one Fallopian tube obstructed and the doctor managed to release the obstruction, we were optimistic, because he said that now we have greater chance with the treatments".

R. Z. - "I was very scared from the injections in the beginning, how do I administer the injections alone? I went every night to a nurse to administer the injection to me and she was very patient and empathetic. A week later I already administered the injections to myself".

P. G. - "In the first insemination I was pretty scared and I was embarrassed with the situation and the nurse held my hand and calmed me down".

S. B. - "I was treated by a private doctor, despite the fact that he was very practical, he was always available for any question and that gave a good feeling".

\subsection{Thoughts}

The waiting period from end of treatment till pregnancy test is accompanied by continuous thoughts, anxieties, and wonderings maybe yes, maybe no and if no, why, what is going on there. It is hard to wait for the expected pregnancy test, as the interviewees tell:

H. C. - "After a treatment didn't succeed, I immersed into negative thoughts, I had difficulties picking myself up, the fear of infertility was very strong".

L. A. - "All day long you think that maybe this time, maybe this time and I wish... and it is a little difficult to function this way".

N. S. - "The thought I have no control over what is happening even though I am doing all I that can be done as far as I am concerned". 
R. Z. - "I thought that life isn't fair and probably I should go through all this in order to become a mother. Life is trying to teach me a lesson".

K. G. - "In the first treatment I have been through I tried to be optimistic, but I didn't really succeed, and it is possible that because of that I did not conceive, I simply transmitted that it wouldn't succeed".

P. G. - "After a failure I had thoughts of why do I deserve it, or why I don't deserve it?"

H. A. - "I had thoughts and hesitations between motherhood and money and every time I think that's it, this will be the last treatment".

S. B. - "I thought I will get pregnant after one treatment and that's it... but it wasn't like that".

H. T. - "The period after the treatment and until the pregnancy test is filled with tension and worries and then the thoughts begin..."

L. H. - "There were thoughts of if it does not work, what's next?"

Whereas five interviewees had positive thoughts, as they describe:

L. Z. - "I was always the backbone of the family, outward as well and despite the crises I have to be strong and not reveal it outward".

K. L. - "The expectation is very difficult, suddenly everything is connected with why yes and why no and when it is negative, I try being optimistic and not to break down".

G. A. - "I was scared whether it will not be successful for me, however I had positive thinking and faith it will happen".

R. G. - "I had hope that the treatment would succeed, and I will not have to continue for a long time".

N. A. - "Every negative thought that came up was suppressed with positive thought or humor, I tried not to deal with it".

\subsection{Emotions}

The emotions accompanying patients in this stage span between expectation the treatment would work and disappointment when the period arrives, and they realize the treatment was not successful. For some patients receiving the first negative answer is very powerful, because they do not believe the treatment can fail. As they describe: 
L. Z. - "As much as I tried and I really tried everything when I received the first negative, I felt like the sky fell over me, here I felt it is not going to be easy".

G. Y. - "Mainly I was disappointed, the wait for the pregnancy test was nerve wracking."

H. C. - "Especially after the first negative I was upset, hurt, cried, I really mourned".

N. S. - "Not easy, as much as I laugh outside, from within I feel a vortex of emotions".

R. Z. - "In the first time when the answer was negative I felt I can't breathe, that I drown in pain and can't deal with it, but mostly I felt alone".

K. G. - "I felt that I will be pregnant within a month and I didn't believe the treatment can fail".

P. G. - "The first negative is more painful, more surprising and darker than anything you can imagine".

H. T. - "There was a tremendous disappointment with receiving the first negative, I had to find strength to keep going".

R. G. - "There is a sense of uncertainty, helplessness and sorrow..."

N. A. - "The expectation the treatment would work gives me sensations of encouragement and cheerfulness, however the moment you receive the negative answer (especially the first) and realize the treatment failed these sensations are replaced at once with bitter disappointment and despair".

L. H. - "The period until the Beta test I was nervous - every little thing annoyed me and I was also troubled however when I received a negative answer I was very disappointed and had no motivation at all".

Whereas four interviewees had positive feelings, as they describe:

K. L. - "There were several hours that it is not nice, unpleasant and even annoying, but not more than that".

L. A. - "Expectation it would happen and then came the disappointment when it does not happen, but the will is strong, so you do whatever is needed".

H. A. - "There were ups and downs in mood, the expectation for a positive Beta is frustrating, however at the same time it gave me hope". 
S. B. - "I tried my best to continue the routine as much as possible and not immerse too much".

\subsection{Patient's actions}

After few treatments that were unsuccessful, patients face two options - to move to more complex treatments (such as IVF), or to continue with current treatment and not go to the next level due to being afraid of the hormones and their side-effects / not continue with any treatment at all at that moment in order to think about it and replenish strengths. Most interviewees chose to move forward with the treatments as they describe:

L. Z. - "The doctor said that after 3 cycles with hormones you get IVF, you receive it from the government after the treatments didn't succeed. The doctor filed a request and I got an approval to start IVF".

H. C. - "Due to the fact I had a thin mucosa after the treatment with Ikaclomin the doctor suggested to move to injections with Gonal".

L. A. - "After 2 cycles with hormones I asked to move forward to IVF, but the doctor said we will try one more cycle and if it won't work we will move on to IVF".

N. S. - "After 3 treatments of injections the doctor recommended to move on to IVF and filled in a request".

K. G. - "After 2 cycles of Gonal I asked to move on to IVF and the doctor filled in a request".

P. G. - "Since there was a difficulty in the insemination to enter with the catheter to the Cervical Canal the doctor suggested we move on to IVF and I agreed".

H. A. - "After one treatment cycle with hormones we decided to move on to IVF due to high FSH".

S. B. - "After two natural cycles the doctor suggested that we move on to treatment with Ikaclomin".

R. G. - "After a number of treatment cycles with Ikaclomin I asked the doctor to move forward with the treatment and he suggested we move on to treatment with Puregon".

L. H. - "After 3 treatment cycles without hormones the doctor recommended that we start treatment with Gonal".

Whereas five interviewees decided not to move forward to more complex treatment or they have asked for a break, as they tell: 
K. L. - "The doctor suggested we move on to treatments with injections, I hesitated a little in the beginning because I read and heard about their side effects and I asked for another treatment cycle with Ikaclomin".

G. Y. - "I went through a number of cycles with injections, the doctor suggested we move on to IVF. I was stressed because I was told it is scary because you inject a lot of hormones and there could be an ovarian torsion, therefore we preferred to continue with the injections for at least two more cycles".

R. Z. - "After one cycle of Gonal and insemination I did a break of few months and returned again for treatment. When Endometriosis was diagnosed, I was suggested to move on to IVF and I even got approved, but before I started I got cold feet because I was really afraid of the big amount of hormones and also from getting fat".

H. T. - "After 2 natural cycles the doctor suggested to move on to hormones, however I was really afraid especially of the side effects, I asked the doctor to continue with few more natural cycles".

N. A. - "After 2 natural cycles the doctor suggested me to move on to treatment with Ikaclomin, however I didn't want drugs, I preferred to take a break in order to think about it". In summary, receiving initial treatment protocol is occasionally experienced as a shock and lack of ability to process the situation and the information. Waiting for results of the treatment is mostly difficult and nerve wracking, and knowledge that the treatment has failed brings about a sensation of despair and frustration.

\section{Coping with the outcomes of the chosen treatment}

Fertility treatments can end in one of two ways: getting the menstrual cycle which indicates failure of the treatment and brings about disappointment and frustration or pregnancy which symbolizes its success and brings happiness and joy. Occasionally pregnancy ends with a miscarriage in its early stages or later, which causes greater anguish from the actual knowledge that such a desired pregnancy that great mental and physical effort was put into it, ending with a miscarriage. Big part of pregnancies stopped developing even prior to clinical evidence of a pregnancy (chemical pregnancy). Following that, most patients have coping difficulty. As the interviewees describe: 
L. Z. - "We started the first IVF treatment, in the first time it didn't succeed and here I really felt the depression like I have taken a blow, it was hard for me however I didn't relate to that, like throwing it aside, I took the treatments with humor".

K. L. - "I went through few treatment cycles with Gonal which didn't succeed. I had to pull myself together, the difficulty went beside me, meaning I'm not inside that point, there is some difficulty and I put it to the side, it is next to me not with me for good and bed".

G. Y. - "I went through 6 cycles with hormones and insemination that didn't succeed, there were many disappointments and my coping was some sarcasm that it will come when it will come, there is not too much to cope with but believe and say everything will be all right and believe it will succeed".

H. C. - "When we've already been before IVF treatment I was conceived, however unfortunately in the $19^{\text {th }}$ week I had an abortion due to Klinefelter's syndrome. I had difficulty making a decision to end the pregnancy, it shattered me to pieces, it was very hard for me to cope mentally - I applied to a psychologist for counselling".

L. A. - "After first treatment cycle I was conceived but the embryo didn't have pulse and I had to have an abortion, I didn't feel like doing daily tasks, it is not simple to overcome. I went to alternative treatments (hot pools) which is very relaxing, and mainly getting out a lot, not to stay at home, go out with friends, go to the family, get the pressure out".

N. S. - "I was conceived in the second IVF but in the $21^{\text {st }}$ week the embryo didn't have pulse, I went through a stillbirth it was very difficult for me, I went into the car in a storm and drove don't know where... all the way I cried and cried. Work really helps me, I like to bake and watch TV, that relaxes me".

R. Z. - "After one IVF cycle I had a chemical pregnancy and the waiting until the Beta will zero down was exhausting. It felt like the experience in the process is slightly similar to the experience of people dealing with depression in certain aspects. When it is difficult for me I detach myself and rest and then find the strengths to move on".

K. G. - "I went through 5 failed IVF treatments and 2 natural pregnancies that didn't succeed, in one I got Prostaglandin and in the second a miscarriage, it was very difficult for me. After every treatment that doesn't succeed, I try to make it up for myself be it smoking, eating mainly things that are forbidden in pregnancy, drinking alcohol and that motivates me for the next treatment". 
P. G. - "After the $3^{\text {rd }}$ IVF treatment cycle there was pregnancy however I lost it in the $7^{\text {th }}$ week there was simply no pulse. I had to go through an abortion, I was depressed and anxious, I did not stop crying it is frustrating and despairing. Sometimes I feel I need to unburden, share, feel sorry for myself for a moment with someone else but I am scared and so ashamed to share".

H. A. - "I was treated in a public hospital and went through 5 unsuccessful IVF cycles. I had many breaking moments when the treatments did not succeed. I did acupuncture and reflexology because I was stressed and that was slightly relieving".

S. B. - "After a cycle of Ikaclomin and insemination I was conceived, a week after the Amniocentesis my water broke, and I had an abortion in Tel Hashomer Hospital. Afterwards I had infection and I was hospitalized again. I had difficulty overcoming the loss, I tried to be busy and do everything on automatic".

H. T. - "I went through 3 cycles with hormones and inseminations and I had about 2 or 3 chemical pregnancies. No doubt it is painful, knocking over and frustrating, however I look at the bright side which is I can conceive and this is extremely important".

R. G. - "After a third cycle of Ikaclomin there was a chemical pregnancy, I had to find lots of mental strengths, I tried to focus on myself and the path before me and made an effort not to see anything else".

N. A. - "After third cycle of Ikaclomin I was conceived but it was an ectopic pregnancy, I was in the hospital and got an injection, I forgot its name the one that you mustn't get pregnant 3 months afterwards, it was difficult to hold the pain, so I tried not to deal with it". L. H. - "After second cycle of Gonal I was conceived but in the $9^{\text {th }}$ week I was treated with Prostaglandin that didn't work and eventually I had to have an abortion and then a surgical hysteroscopy because there was a residue of placenta. I had difficulty after the abortion, I took a week off from work, because I couldn't cope mentally. I ate, I watched TV series and mostly rested and occupied myself with things I like".

\section{1 - Support systems}

Many a patient feels ambivalent regarding sharing with family / friends / the close environment / work colleagues and even the spouse what they go through. On one hand, they desperately need support and understanding and on the other hand they might come across 
frustrating or hurting responses. As they are in the midst of a crisis, they are especially being hurt by responses perceived by them as indifferent or insensitive.

\subsubsection{Israeli society}

There is a tremendous pressure from the society on women to do everything for a baby even when the chances are extremely low. Despite the fact that it expresses sorrow and support for failure of treatment or loss of pregnancy, at the same time they push to continue despite the difficulty, to such an extent that there is no cultural possibility to choose avoidance of treatment or end it. As the interviewees tell:

L. Z. - "They look at me with pity, it hurts".

K. L. - "I was told what are you crying for? There are more difficult cases".

G. Y. - "I was told when you will not think of it it will come and that makes me very angry".

H. C. - "I was told, Ohh, so sad, what would you do now? What can I do? It is difficult for me".

N. S. - "They tried to cheer me up and say - believe that anything that happens is for the best, think that if something didn't develop there must be a reason and that really offended me". P. G. - "Some of the people who heard I went through a miscarriage said - don't worry, it happens to a lot of women, it didn't really comfort me".

R. G. - "Some people told me don't take it too hard, this is not even a pregnancy, there are many women who were slightly late and they didn't even know they were pregnant and later got their period as usual".

N. A. - "Some people told me, well, all right, so wait for 3 months it's not a big deal you are young, try again".

Whereas seven interviewees told they received support from society:

L. A. - "I was suggested to go abroad and take a break in order to relax and replenish strength".

R. Z. - "I was given advices and told stories about girls who succeeded after so and so treatments and that has given me motivation".

K. G. - "Some people encouraged me to continue despite the difficulty."

H. A. - "I was told, don't give up, every negative makes the sought-for yes closer." 
S. B. - "I was told, take a deep breath for yourself and organize for yourself a schedule back to treatments, don't wait for months".

H. T. - "I was suggested to ask the doctor for Thrombophilia inquiry and additional tests".

L. H. - "I was told they say the body is very fertile in a month following a miscarriage".

\subsubsection{The family}

The family not always knows how to behave during treatments / loss of pregnancy. They do not know whether the preference is raising the subject and asking or to respect the privacy of patients. They are not sure whether it is desired they encourage, advice or comfort as occasionally it might be interpreted as prying or even as hurting due to the sensitive condition in which the patients are. As they tell:

G. Y. - "The family asked what happened to you that you got so fat? And then touched my stomach and asked if I was pregnant".

H. C. - "It was difficult for me to go to family meetings I didn't want them to pity me".

N. S. - "I didn't want their pity looks and to be treated as I am unfortunate I reduced the meetings with them".

R. z. - "My little sister announced she is pregnant, I felt like the whole world is closing on me, I couldn't stop crying for a week".

K. G. - "Despite my mother knowing I am in this experience she still is tactless of telling me about everyone in the family who is pregnant".

P. G. - "My mother told me you shouldn't be jealous, your time will come too, here so and so waited for several years and at the end it came for her, I really felt upset".

H. T. - "They try to encourage me with statements of never mind, chemical isn't even a pregnancy and that hurt me badly".

R. G. - "We seldom met, we had nothing to talk about."

L. H. - "My family are not the most inclusive on the face of the earth."

Whereas six interviewees told they received support from their family:

L. Z. - "My mother said to me don't break down, get up and pull yourself together and one day it will happen". 
K. L. - "My mother said she believes that things happen for a reason and it's a matter of time, we shouldn't lose hope".

L. A. - "My mother said, apparently it wasn't all right, it's better it happened now and not in a later stage".

H. A. - "My mother said stress and disappointment influence absorption capability, limit the time of upset".

S. B. - "I told my mother I've been through an abortion and to my surprise I got from her hugs, affection and support".

N. A. - "After the miscarriage my mother tried to approach, however not knowing how to do it".

\subsubsection{The spouse}

Occasionally, each of the spouses receives differently the disappointment that at that month the expected pregnancy did not happen as well. It is not always easy to break down near the other party, or share the hard feelings without sounding accusing, disappointed, frustrated, despaired or scared, and thus sensation is created that the communication was damaged and it is not possible to discuss the truly burdening matters with one another and it is easier to share it with others. As four interviewees describe:

L. Z. - "He doesn't really understand that it is hard for me, he helps with everything but concentrates more on how it is being hard for him".

K. L. - "As far as he is concerned, one month more or one month less is not that important, as far as he is concerned the hormones, the drugs, the follow-ups and the tests is not that bad, it's something to get through".

H. C. - "My husband couldn't talk, he just introverted and found it difficult even to comfort me".

L. A. - "My husband is very closed and introverted and he really wants me to be pregnant already, although he does not open emotionally, I know it is hard for him too".

Whereas one interviewee told that the male spouse supported her:

G. Y. - "Cheering one another that all will be all right".

Amongst interviewees that have female spouse, most received support, and inclusion from the female spouse as they tell: 
S. B. - "My spouse was always there for me along the entire process, comforting, hugging and calming".

H. T. - "The spouse is very supportive and hugging, encouraged me to continue despite the difficulty".

R. G. - "The spouse tried to soften and round corners and cope efficiently with the entire situation".

N. A. - "The spouse expresses a lot of empathy for my pain and difficulty, very inclusive". Whereas one interviewee told she did not receive the support she expected to receive from the spouse:

L. H. - "Despite the fact that the spouse is supportive and helpful, however it was hard for me that she regarded the situation lightly".

\subsubsection{Friends}

At this stage some friends hide the fact that they are pregnant in order not to bring sorrow or that they are afraid of ill-luck, some tell about it without knowing it is extremely painful and hurtful especially when the patients struggle to conceive. At times a certain distance is created even up to disconnection and there are friends who still support and encourage in moments of crises. As they tell:

L. Z. - "Lately she avoided me and I didn't understand why, when the whole group of friends set a meeting she arrived with a fifth month belly going in front of her, apparently everybody knew but me because they didn't want me to get hurt".

K. L. - "Friends tell me cheer up, everything is all right with you, but if everything is all right how come there is no pregnancy?"

H. C. - "My best friend, the one that goes through this journey with me maybe more than my husband, found out she was pregnant and told me. Of course I was glad for her, however I was sad for me for the injustice for how much she was upset because she didn't plan the pregnancy. And me? I fight like crazy, I felt like my soul is burning, unfortunately I lost my journey companion".

N. S. - "One friend told me what do you need it for, you wreck your life and your body." R. Z. - "My best friend is already in the sixth month and all during her pregnancy didn't stop sharing me in every detail and this hurt me because I felt I try to suppress and she kept 
reminding me again and not out of bad intention but out of a habit that we shared everything with each other - gradually I grew apart from her".

K. G. - "A friend called to tell me, I beat you, I couldn't understand what she was talking about and I asked her in what? And she answers, say congratulations, I'm pregnant! I was in shock".

H. A. - "I used to go to events, baby celebrations. Until one day a "friend" put me at a table with all the gorgeous babies and I thought I would pick her eyes out. You knew I am after an unsuccessful cycle; you could be more considerate. Anyway, she is no longer part of my life (and that is for the better) but since then I took distance a little and went only to the people who understand me".

S. B. - "After the miscarriage I was gone through it was hard for me to go to meetings with friends because regardless of how supportive they are, they will give you the feeling of some misfortunate one, and this feeling will accompany you like a cloud during entire evening".

N. A. - "I usually go to such events... once in a while, when it feels that the only interest will be on the subject of children and I will be expected to be the nice aunt to everybody else's little ones, then I choose not to go".

Whereas six interviewees told they received support from the friends:

G. Y. - "A friend advised me to take some air, breath deep and move on".

L. A. - "A good friend took me for an alternative treatment that really relaxed me".

P. G. - "A friend encouraged me, nothing from what you did could prevent the miscarriage from happening".

H. T. - "A friend encouraged me that it is all right to be sad but most important is not to look back because this is not where I am going".

R. G. - "A friend tried to encourage me and told me that with her the first Beta was low and then it raised a little and the doctor said there is still a chance and today she has a child".

L. H. - "This is not easy, but you can't run from it, the friends didn't let me sink into depression and sadness".

\subsubsection{Work}

Not all employers accept the fact that their employee is in treatments, due to absences from work, possible hospitalizations, etc. On the other hand, the patient is occasionally forced to 
work under pressure and stay overtime after absences due to the treatments. According to law, absences from work due to fertility treatments considered as sick days anchored in law if a patient gives advance notice. As they tell:

L. Z. - "As treatments went on I found it difficult to miss work days, there was no flexibility because I studied and my boss didn't always receive it with understanding, especially when I didn't give advance notice".

K. L. - "I refused a promotion at work that requires more responsibility which equals more pressure, it is already hard for me to maneuver with the tests and treatments".

L. A. - "Despite supporting me in a personal aspect, in the professional aspect my manger has difficulty letting go when I took a sick day, I got sour face more than once".

R. Z. - "Generally I received support, but I was given the feeling that they do a favor for me, that they give me a day off out of obligation and not wholeheartedly, sometimes I had to make sure I had a substitute and it wasn't easy with the burden of treatments".

K. G. - "I am not happy with my work and I am concerned that my unstable mental state influences getting conceived, on the other hand I am afraid to get kicked out from work".

H. A. - "The pressure at work is sometimes really bothering and sometimes I feel nervous and about to explode".

R. G. - "There were times I had to cancel work and somehow that harms because we are less invited".

L. H. - "I feel burnout at my workplace, and I want to quit, however on the other hand I can't do that in the midst of treatments, I and in a complicated dilemma".

Whereas seven interviewees told they received support from the working place:

G. Y. - "I managed to maneuver with shifts, sometimes I would say I had a test because the boss is very strict".

H. C. - "Meantime I didn't let know at work that I am in treatments, I managed to handle somehow, I still hesitate to tell or not to tell, a little fear for my workplace".

N. S. - "I actually like going to work because that keeps me busy, keeps my thoughts off between one test and the other, including waiting for the Beta, it occupies me positively". P. G. - "I always said I had errands and I will arrive later. I completed the hours or stayed longer or arrived in the day off so they will see as less absences as possible". 
S. B. - "Luckily my position is flexible, and I reduced working hours."

H. T. - "I tried to be above and beyond and nearly not be absent at all, I felt obligated to the workplace".

N. A. - "In the first months I managed and then they started to realize I am not just missing work, they conducted a hearing to me and I had to tell I am in treatments, gladly they accepted with understanding".

\subsubsection{The medical system}

The medical system at times creates hardships for a patient, especially bureaucratic ones, which make the process harder. Some patients do not receive empathy and sensitivity from the system, which causes patients to be tossed around and frustrated.

As they tell:

L. Z. - "They came and said listen, you have a delay because the lab technician, which happens once a million, dropped your embryos, I said what now, I will not wait two more month, they said indifferently there is nothing to do about it, they were not even sorry".

H. C. - "Because my husband was in a different HMO we had to run around and do the sperm enhancement in one place and the insemination in another and if we wanted one place we needed to pay a substantial sum".

R. Z. - "The HMO did not enable me to do continuous egg extraction, but demanded a break of 45 days between one extraction and the next. The meaning is skipping a treatment cycle. In my age every cycle is significant".

K. G. - "In the last embryo transfer the doctor who did the transfer said, well, it doesn't look like it is going to succeed, there is not too much to hope for, and that naturally cracked me." P. G. - "The doctor forgot to file a request for IVF treatment and therefore I couldn't start a treatment, unfortunately I had to wait for the next month".

H. A. - "One of the professors told me indifferently, your chances ae low and egg donation is your option, you waste precious time, think it all over, there is no point to continue".

H. T. - "We went to a super professional doctor; however, he is unavailable, doesn't answer his messages and when we do reach him he gives me a feeling I am bothering him and claims he is busy". 
S. B. - "Sometimes there is a complex bureaucracy of the medical system, to chase after approvals sometimes in the last minute and that is extremely stressful".

L. H. - "Lots of frustration with the medical system, I went to alternative treatment".

Whereas six interviewees felt they have received support from the medical system, as they describe:

K. L. - "The doctors look at things one way and the nurses in a different way and this combination is very important, sometimes I leave the doctor and ask what does he want? And the nurses have more patience and that is much more important".

G. Y. - "I felt I am in good hands medically, the discussions, the smiles, it's very encouraging".

L. A. - "Once a doctor around here told me, what are you worrying about, you will still have lots of children, and that is something that was imprinted".

N. S. - "I have received a lot of mental support from nurses in the clinic, they embraced me".

R. G. - "The nurses were very kind and looked for creative solutions to the problem I had".

N. A. - "The procedure was very long and despite that the doctor was very patient and gentle and kept caring for my wellbeing".

\subsection{Thoughts}

A thought that a treatment will bear success brings hope that is proven false as soon as the pregnancy test is negative, which causes disappointment. Uncertainty of the process and its outcome brings about frustration and there are patients who in advance have negative thoughts so that they are not disappointed, as they tell:

L. Z. - "It felt like you are going to war that you don't know what to expect from, meaning it could last two more months, one year, two years, three years, 10 more years, no one can really tell you when it will happen, feeling of helplessness".

G. Y. - "When it is negative even when there were good indications you ask yourself, what is the reason it didn't work, and you are in a kind of uncertainty".

H. C. - "Since the miscarriage the jealousy makes me mad, I said to myself, wow what a mean nasty wife I had become, what is going on with me? What have I turned into? It is hard." 
L. A. - "I felt lack of control, that everything is taken away from me, what I am aloud to eat, to drink, when to have sex with my husband. All day long I am being stubbed, examined, I have no longer control over myself and no privacy".

K. G. - "The most difficult thing is the uncertainty if it is ever going to work, if I will not see a negative for once and finally succeed. Eventually I blame myself".

H. A. - "The thought and the heartbreak every month all over again, why for others does it come so easily? I try to have high hopes and expectations so that I am not disappointed".

S. B. - "The most difficult thing is there is no way of knowing when the journey will end and how it will end. The thoughts of why did it happen? God, I can't figure it out".

H. T. - "The uncertainty of when it will finally come, ends me off, the wonderings and this hallucination that I have no fertility problem and it still does not succeed freaks me out".

R. G. - "When I was told of a negative - they did tell me in ultrasound that the mucosa is nice and ready, and I have a few pretty follicles and still it didn't work. Why do I of all people deserve this?"

N. A. - "I felt ashamed, this exposure to the whole world, I'm touched, looked at, observed, I am exposed..."

L. H. - "Why did I have to crush and hear there is no pulse? Why is it so hard and long? It is frustrating".

Whereas four interviewees had positive thoughts:

K. L. - "It is a little frustrating because there are moments of crisis, but they passed because I try to think positively".

N. S. - "I know I will have a child; I am aware it is sometimes hard to imagine especially now when I received a negative answer after a long and hard treatment, and it is a little difficult to see ahead".

R. Z. - "I am glad because I know the goal is good and worth it all and sometimes, I feel sad because I go through the process alone and that is not simple".

P. G. - "When another fertilization failed after it took me 3 hours to go to sleep and the pillow was soaking wet, I realized that besides trying and trying I can't do much more". 


\subsection{Feelings}

The medical drug treatment that patients receive might cause to some of them mood fluctuations and when the expected pregnancy does not come after many treatment attempts and after a long time of expecting, hoping and waiting, there is a feeling of pain, emptiness, loneliness and frustration that enhance when loss of pregnancy occurs.

L. Z. - "The hormones dosage affected me, I was swallowed, nervous, frustrated. I cried without reason, I got scared from myself. I don't know myself this way".

H. C. - "Entire nights I was so agitated from sorrow, feeling of emptiness, failure and also a feeling that the body betrays you not to mention fluctuations in mood because of the hormones".

L. A. - "Sadness and happiness at the same time and it is not simple and not controlled due to the hormones and yes, there are more moments of crying and sadness".

N. S. - "When the doctor said there is no baby's heartbeat, I said naively that maybe the device is broken and that he gives me another place the check it, it shattered me".

R. Z. - "That is exceedingly difficult. I never felt so lonely, it's frustrating and I am angry with myself for wasting time".

K. G. - "I felt stupid that I was so happy and made plans and already told everybody and suddenly there is no pulse... simply a heartbreak".

P. G. - "There were many moments it felt like a dark hole of despair, hardship and loneliness. I feel like I am fighting alone and that is not easy".

H. T. - "Chemical pregnancy brings high hopes, in the beginning there is proper double and there is a belief that it will still be all right and then comes the slap on the face and again all the dreams go down the drain and the heart shatters".

N. A. - "Sometimes an uncontrolled crying due to the hormones, not feeling like doing things, sometimes I close for a day or two with bitterness and also a sensation of miss that it doesn't happen".

L. H. - "18 days since the insemination I decided I will not do a pregnancy test out of despair; I have a fear that if I am pregnant I will have a miscarriage again". 
H. A. - "When going through treatments you must believe it will be all right, but when you only see failures one treatment after another and the doctors don't know what to tell you, a feeling of certain helplessness".

S. B. - "A challenging and complex process, exhausting mental powers accompanied by many tears, disappointment and sometimes frustration, the hormones simply freak out and sometimes there is no control".

Whereas three interviewees describe positive feelings:

K. L. - "There are moments of mood going down, it is a matter of few hours and I need to pull myself together. There is no other choice, this is a process and it ends".

G. Y. - "I have moments I feel sad anyway, I am emotionally overflown, but generally I am a positive and optimistic person".

R. G. - "There are mood fluctuations, anxiety and tension, I cried for two days for each failure, however the goal is worth it all".

\subsection{Actions of a patient}

Fertility treatments are mentally exhausting, however despite that, most patients have chosen to continue or move forward with the treatments or they changed doctors in order to try another treatment and some have chosen to take a temporary break from treatments in order to regain strengths for the following. As they describe:

L. Z. - "I wanted to continue with the treatments, I really wanted it happens already".

K. L. - "I decided to move forward to IVF because I have the right to use those treatments and be somewhere else".

G. Y. - "After 6 cycles with Gonal F that didn't succeed we decided to move forward to IVF although I was really scared from the procedure".

L. A. - "We decided to continue despite the difficulty".

R. Z. - "Despite the fact that this is very difficult for me I continue with all I have because I don't have a lot of time".

P. G. - "Despite the difficulty I keep trying and not giving up".

H. A. - "I decided to change to a private doctor who will go along with my wishes".

S. B. - "It is hard to overcome the loss however I knew I have to move on". 
H. T. - "We decided to try another hospital out of a thought that there might be another way". Whereas five interviewees have chosen to take a temporary break from treatments, as they describe:

H. C. - "I had a feeling of guilt with my body and I had to deal with this grief which caused a crisis in our relationship, so I decided to take a break in order to care for myself".

N. S. - "I took a break of few months in order to mentally recover from what I've been through".

K. G. - "After what the doctor told me, I wanted to dig in inside my bed and not to get out of it. I found it difficult to continue".

R. G. - "I felt mentally exhausted and that I need rest".

L. H. - "It was hard for me to recover after the miscarriage and I decided to take a break in order to fill up the batteries".

In summary: the patients find themselves in demanding fertility treatments route which dictates new daily routine including medical drugs, tests and procedures (IUI / egg extraction and embryo transfer) that can end with failure of treatment or with pregnancy that is occasionally abnormal and can cause upset and disappointment.

\section{Being in a treatment}

Fertility treatments become more complex, cumbersome, and exhausting and they might take a long time and cause some patients a despair. The treatments require a lot of patience and difficulty in maintaining continuation of life routine due to a tendency to put fertility treatments at the center of life. Longing for a child and the sense of helplessness causes many a woman to address a "supermarket of treatments" (different and peculiar diets, holly people, alternative medicine) out of a desire to verify that they have indeed done all within their power to conceive, as they tell:

L. Z. - "I really tried, and I can tell you I also went to Rabbis and went to all sorts of healers who told me to drink things and eat things, whether to keep a diet and truthfully I didn't conceive".

K. L. - "Life revolves around the treatments, blood tests and ultrasound, egg extraction with full anesthesia a complete day of disconnection from life, from work... these treatments cost 
me a lot of money that I didn't always have, but I kept going again and again, because as far as I was concerned it was a bright spot for me".

G. Y. - "We gave up other things, our priorities have changed, not flying abroad, no house renovations. All our resources were directed to the treatments".

H. C. - "We decided to try everything with the purpose of succeeding - Acupuncture, Pipelle, all types of hormones existing in the market, but nothing helped".

L. A. - "At some stage I was desperate, I drank Vitex tea, I drank Wheatgerm, I ate beetroot (one of my most hateful things), I read Psalms, I told the girls to prey for me and I asked for the blessing of a godfather".

N. S. - "Hard demanding treatments, damaging daily routine and as a result I felt high levels of stress and distress, harm to my self-image and the daily functioning".

R. Z. - "You constantly are in the midst of a hormonal protocol that disrupts who you are, all life circles around the extractions, the transfers, the tests. On the road there were moments of crisis and despair, but it passed, because I felt I was in a mission, the children I took care of were sort of comfort".

K. G. - " The treatments were a journey. I decided I try everything - I visited Rabbis in Zefat, a witch doctor, I had cupping glass done to my uterus, I was at color diagnostician, psychotherapy, went to a health farm to clean my soul".

P. G. - "Total enslavement, I had no life besides working at it all day long. Even if it was sitting for hours in ques".

H. A. - "In my case all my life circles around the treatments, they manage me completely - I couldn't plan in advance working abroad because I will not fly with injections and I don't know when the fertilization day will be and the IVF currently is more important to me than anything else".

S. B. - "I was so crazed about conceiving and I didn't notice what it was doing to me and the effect of hormones is stronger than in pregnancy and that makes everything more complex, in order to function you should tell yourself it is not you, it is the hormones".

H. T. - "I devoted myself to the treatments, both out of lack of time and lack of desire to deal with other things. My life circled around the fertility treatments, the hormones, injections and waiting for phone calls from the nurse". 
R. G. - "Expecting a child took over all areas of life and was expressed also in daily things, my life was on hold".

N. A. - "I had a tendency for crying, lack of enjoyment from things naturally enjoyable, sleeping problems and nervousness".

L. H. - "This race took over my life, I would find myself dealing only with that, thinking only about that, nothing is interesting, and nothing matters anymore".

\subsection{Support systems}

The physical, technical, and emotional difficulties accompanying fertility treatments naturally increase the need for support from the people around. Despite that, occasionally, when treatments take long time they are in fact accompanied by loosening of significant social and interpersonal relationships (such as family, friends, work colleagues and close environment), and also intimacy might loosen as well following the continuous stress that might damage intimacy performance".

\subsubsection{The society in Israel}

The pressure on women to conceive increases even more as all the means are at their disposal and all they must do is use them, while ignoring the difficulties, suffering and the high cost they have to go through. Women who are in the midst of the process feel they are destined to sink into an abyss of despair if they do not give birth to a child, as they tell:

G. Y. - "If you are stressed take Rescue, it is not as bad as you think, it was hard for me to hear that".

H. C. - "Get back to the treatments why are you still crying? This is not yet a baby, you didn't have the time to get attached to it".

K. G. - "I was afraid to give up, because I was afraid to be invisible, not to be part of the society".

R. G. - "I was asked, are you committed to the treatment? It does not fail if you are stricter regarding nutrition".

Whereas 11 interviewees were encouraged by society not to despair, as they describe:

L. Z. - "Don't give up, you will be a mother. A lot of women give up and let go and conceive, all is for the best". 
K. L. - "The hard part is just the anticipation of an answer, breathe and accept it with love with the hope that this is a one-time process".

L. A. - "No way you despair and give up, you are allowed to rest a little, breathe and return with replenished strengths".

N. S. - "Believe and believe it will come, the road is hard but it is worth everything and you forget every moment of pain / disappointment".

R. Z. - "I was given a message that if I am strong and determined enough I will succeed".

P. G. - "I felt it was too heavy on me and I thought of giving up they told me, take a break don't despair, you have waited for so long, wait for two more months".

H. A. - "Every time I was about to give up I was encouraged not to despair and continue with the treatments because eventually it succeeds".

S. B. - "Take a vacation abroad / shopping, it helps to fill up the batteries".

H. T. - "Ask for another treatment protocol, that might help."

N. A. - "Think positive it will be positive, as soon as you are a mother you will forget the hardships".

L. H. - "Think that every treatment is a light at the end of a tunnel, that gets you closer to your goal".

\subsubsection{The family}

Overtime, the couples become more and more isolated, they might stop sharing with the family in order to not raise expectations and hopes that would put them before a new round of questions, it is possible they will avoid family events, which might expose them or raise hurtful feelings. Sometimes family members drift apart and feel hurt from the lack of inclusion.

L. Z. - "We stopped sharing with the family treatment appointments, so they do not develop expectations. It adds to the pressure when they know, because they should be updated, and they are disappointed for you".

G. Y. - "I really hated that everyone tried to cheer me up and say all the time, it is going to be all right, I wanted to shout at them, who said it is going to be all right?" 
H. C. - "The most difficult thing for me to hear was - eventually everyone succeeds, they tried to help in their limited and not necessarily useful way".

L. A. - "We gave minimal information to the family, anyway every time I saw someone pregnant in the family I would cry to my husband and he wouldn't know what to do with me. Even though I already have a child it still hurts".

N. S. -"It was extremely hard for me to go to family events, all the brothers and sisters in law with children are a reminder to the fact you haven't succeeded yet and that hurts. so, I avoided going".

R. Z. - "Meetings with the family were hard because my life is in a loop between despair and hope and it is difficult to put a smile and relaxation on your face the whole time."

P. G. - "I asked them to stop pitying me because I am not poor, however, to do it in the right dosage... nobody really understood, they were offended".

S. B. - "When the family made an inappropriate remark, I responded to them, they should take into consideration and with understanding the fact that their advice will not always be accepted, it offended them a little, but what could I do?"

H. T. - "They (the family) should exhibit patience towards my alternating moods, I tried to explain to them that it is not them, it's me, they were offended".

R. G. - "I moved away from the family because it helps me retain my sanity and does not take additional energy out of me".

N. A. - "The family didn't show interest, didn't ask, didn't offer help and didn't support in any way whatsoever, and I was really hurt by that, as far as I am concerned, they exhibited indifference".

L. H. - "The family didn't accept my unwillingness to come to events in which there were children".

Whereas three interviewees were supported by the family, as they describe:

K. L. - "I have a great supportive family however there are difficulties which are only mine that you can't share with anyone else and no one can understand, help or make them easier". K. G. - "I asked not to come to family events because it is hard for me. It was difficult for them to understand, however in most cases they respected my will". 
H. A. - "Indeed, my mother would come with me to the extraction, however I saw on her face that it is difficult for her that it doesn't work out".

\subsubsection{The spouse}

Spouses are required to deal with difficult feelings of pain, disappointment, guilt and more, and at the same time to deal with the demanding treatments and all energies are channeled to conceiving. At times it is even very difficult to raise for discussion options such as taking a break from treatments or giving up intercourse when "it is needed" however not desired, as these options might be interpreted as lack of "real" of sufficient desired for a child. The man sees and suffers for the wife and "absorbs" her anger and frustrations, which are not typical to his spouse and occasionally instead for them being a source of support and relief for one another, the difficulties might cause distancing, disagreements and mutual accusations, as the interviewees tell:

L. Z. - "I drifted and I took my husband with me, the moment I failed I wanted more, I couldn't see anything but conceiving until my husband took me for a talk and told me, enough let's take a break, I answered: is that what interests you? I will be a mother and I don't care about anything else".

K. L. - "Normally he is super supportive, caring and what not, however since we started the treatments it is difficult for him that it did not come naturally, it created in him some sort of remoteness from the process".

G. Y. - "For him everything was cool, everything has a solution, like he can't understand what the problem is. He doesn't understand deeply what I experience mentally and physically".

L. A. - "I was hormonal, and it was difficult for him to contain my angers and my crying outbreaks because it is not typically me".

Whereas H. C. described the situation from a positive viewpoint, as she describes:

"I slowly realized it is difficult for him and he simply chooses to deal with it differently than me, so I lowered my expectations. Generally speaking, he makes an effort, we went to a vacation together to refill our batteries".

Opposite to them, all those interviewees with a female spouse reported on support and inclusion from the spouse, as they describe:

S. B. - "My spouse is quite aware of my feelings and very inclusive, pampers me after every unsuccessful treatment". 
H. T. - "There were once in a while disagreement, but not a big deal. In general, she has lots of patience despite all my outbreaks, she is simply inclusive and takes everything with love".

R. G. - "Support from the spouse and her love in the difficult moments held me also mentally".

N. A. - "The spouse pulls me together at times of crisis, gets me on my feet and gives me strengths to continue".

L. H. - "There were anxieties and fears in the beginning, however we are strong together and overcome every obstacle in our way, we do not give up easily".

\subsubsection{Friends}

Friends tend to disappear as the way prolongs and becomes more complex and not out of their fault, as it is difficult for them to cope with a friend who changes in front of their eyes and becomes someone else who is motivated, with a "tunnel thought" directed only towards one direction. Conversations are no longer same conversations, areas of interest are not similar anymore and there is nothing to talk about going out. This way, women narrow the social circle they usually get support from. As they describe:

L. Z. - "On the way to work I met a childhood friend we haven't meet in years, she stood before me, raised her hand and pat my stomach and said - a boy or a girl? I burst into tears as I never did".

K. L. - "I was the one drifted apart from everyone, I see on that Facebook they meet for a birthday and they don't even invite me to come".

G. Y. - "While you are in the process it feels like everybody is suddenly pregnant except for you, and what about the questions? As if it is not enough they also ask, as if we don't want to".

H. C. - "I lost some friends because of the nerves and my mental state, they couldn't contain what I go through".

N. S. - "I didn't go to meetings with friends because no matter how supportive they are there is always the feeling of how misfortunate and it accompanies like a cloud entire evening".

K. G. - "Slowly relations with friends loosened, they are busy with their families and they don't have the patience to hear me complains and natter". 
R. Z. - "A friend told me she is pregnant, I congratulated her, but she said she decided not to keep it. Why, why are you telling me at all? I felt hurt".

N. A. - "A friend I went to school with told me she is pregnant and that ripped my heart especially that a few days earlier I got my period".

H. A. - "Friends of mine who got married and have children organized a party and told me, come if you want to, on one hand I feel distanced and lonely and on the other hand I don't want to sit there and have pain like a blow to the stomach".

L. A. - "Mostly I went to social meetings, every time I heard another friend of mine is pregnant, I let myself hurt a little but I made an effort to think positively".

S. B. - "After unsuccessful last extraction I really sank into depression and I hated myself and with some good friends I managed to recover and move on".

P. G. - "When we had meeting with friends we had an agreement - we do not speak about pregnancies, births and what is between them (about sex yes)".

H. T. - "Friends encouraged me that without these treatments it will not be realized, even if it comes with difficulty".

R. G. - "Good friends cried with me regarding every negative and laughed from my bad jokes regarding the treatments".

L. H. - "Meetings with friends makes us feel good because there is good atmosphere, the support is being felt and they give tips for continuation of treatments".

\subsubsection{Work}

Many an employer is very supportive in the beginning and throw promises that they will help as much as they can. However, when work accumulates and it starts to hurt in the pocket they start backing down from their promise and when they find out they cannot fire the employee as women labor law protects her, occasionally employers look for excuses like mistakes of distraction, or not managing with the tasks etc., in order to fire the employee or alternately to worsen the conditions, (like not awarding promotion), so that the patient leaves on her own will. As they describe:

L. Z. - "I was summoned for a talk with the in-charge who told me I am distracted and not focused on work and later she said, and now forgive me I have to go to my children". 
K. L. - "I really wanted a career change however I was afraid to do it during treatments, I realized it will require additional energies that I felt is more important to invest in the process".

G. Y. - "I was supposed to get a promotion at work, but eventually they decided not to give it to me due to my frequent absences and I am thinking about leaving".

H. C. - "My boss saw me occupied with my phone at work and took me for talk and said, why are you in your phone all day long? Keep it at home. I told her I have a life so she said there are people here with children and I can understand, but you?"

L. A. - "At work despite the substantial pressure time does not move, I look constantly at the phone with tension and it is hard to focus and I have to manage my tasks, my boss already reprimanded me once".

R. Z. - "When I told my manager I will not come on Sunday because I have an extraction she asked - can't you do it on Tuesday? (my free day)".

K. G. - "Lately my manager fights with me on every turn and says I cause problems and he can't do it anymore, says that either I work there or not, he acts like he is doing me a favor that he gives me a sick day for an extraction".

P. G. - "When I decided to share with my boss that I am in treatments he said, tell me so I can train someone in your place".

S. B. - "There were days when I was distracted whether because of side effects or the thoughts of what will be and I couldn't perform at work".

H. T. - "Just when I started additional IVF cycle I was supposed to go to a job interview, I called and said I will not come. I gave up, I don't want to be under pressure in a new job". N. A. - "A new manager arrived who didn't like this issue that I am in treatments and really harassed me and therefore I had to leave the working place with a very bitter feeling".

L. H. - "I was fired from work with claims that I was not concentrated. Due to all the thoughts and the mess in my head, I started to make mistakes and I wasn't there".

Whereas three interviewees felt they were supported by the workplace as they describe:

N. S. - "I feel I am all the time on the verge of crying, however work gave me strengths to continue because otherwise I couldn't function". 
H. A. - "My partner helped me in the days I've been through extractions and enabled me to go home early when I felt I am not concentrated enough".

R. G. - "I had the possibility to reduce some of the work pressure due to the stress of treatments".

\subsubsection{The medical system}

The exhausting bureaucracy of the medical system occasionally lays difficulties before patients, occasionally patients come across insensitivity from the caregivers and the physical dependence upon doctors at times has heavy mental toll.

As they describe:

L. Z. - "I was frustrated already, and the doctor couldn't explain to me why it does not succeed and what can be done so it does happen".

K. L. - "I came across an antipathetic ultrasound technician who examined me unwillingly, at the end of the examination she through in the air, your follicles are still small, and that upset me all day long, I was stressed because of her that I inject hormones for nothing".

G. Y. - "I called the clinic to make an appointment with the doctor and the secretary said there are no appointments, I begged to see the doctor because otherwise I would miss a month of treatments she answered indifferently, there is no possibility, only if there will be a cancellation".

H. C. - "I would come in the morning for tests and around me pregnant women were sitting, it was not easy, my heart was broken".

L. A. - "The doctor doesn't want to move me to IVF, insists that we continue with inseminations, and I really want to move to IVF because I have no more strength for disappointments".

R. Z. - "After the extraction the nurse in recovery saw that only 3 eggs were extracted and said, what is that? Is that what you managed?"

K. G. - "The secretary called me in the day of the transfer and announced me indifferently that I have no reason to come and the embryos didn't make it, asked me to make a doctor's appointment even didn't let me react and immediately hanged up".

H. A. - "The nurse who took my blood suddenly told me, I see that you are here for a while, I want to tell you that you are wasting your time and effort, children are ungrateful. I see that 
you suffer from the treatments and I had to tell you I think the effort is not worthwhile for you".

S. B. - "I've been through extraction of 18 eggs, two embryos were transferred and when I called to check how many cryopreserved embryos remained they told me none, wished me luck and hanged the phone. After I screamed it turned out they were wrong, and I have 7 cryopreserved embryos".

R. G. - "It once happened to me that I did tests and they didn't get back to me to give me instructions, needless to say I was stressed, and it was very difficult to reach them on the phone. I had to miss a treatment cycle".

L. H. - "There were times it was hard to get the clinic on the phone, so we were forced to physically arrive to the clinic".

Whereas four interviewees felt that they were supported by the medical system, as they tell:

N. S. - "I went to update the nurse that I start another cycle of IVF and I was in a good mood, suddenly the tears came I don't know why, maybe because I saw many pregnant women and then the nurse offered me a comforting hug".

P. G. - "My doctor told me in the most difficult moments remember my words - it will be fine in the end".

N. A. - "Most of the communication was done through the nurses who were very professional, sympathetic and answered every question pleasantly".

H. T. - "I was broken from the treatments and the nurse offered that maybe I should take a break this month in order to recover and offered a psychosocial support, although I refuses the support, I took the break because I needed it".

\subsection{Thoughts}

The longer and more complex is the process, thus the difficulty increases and projects on every possible field. Within that difficulty patients often feel that no one can understand or perceive the intensity of pain and the feeling of void and absence, even if she tries to explain. Some try to be optimistic and some find it difficult and have thoughts that the anticipated pregnancy will never happen. 
L. Z. - "I feel I have no strengths for another treatment. I feel that the pregnancy is distant, and I want to believe it will happen so much, I want to be a mother so much, to hug and kiss my small boy or girl".

H. C. - "When it fails thoughts arise in me of why us of all people? Is this the universe's test of our intimacy? The race for a child, are we strong enough to survive it?"

L. A. - "Every time I begin to give up I try to see the positive in life, I have a child and his biggest dream is a brother / sister and he keeps asking when will we have a baby? I restrain myself from crying in front of him, try to cheer myself up that I still have more time but time goes by and hope slowly runs out".

R. Z. - "After a negative answer, the first thing I thought of is that I will have to go back to injections. The moods, sicknesses. Generally, hormones put me into bad situations".

K. G. - "The head is filled with thoughts, what will happen if they extract a small number of eggs, what if it doesn't succeed again? Harsh feelings that you can't see the end of it".

P. G. - "The main problem I find difficult is the stress of time, the fear there is no time left and if it does not succeed now it will never happen at all and every failure makes me sink into terrible depression".

S. B. - "The lack of success, the sensation that something is wrong. The fear I will not realize motherhood".

H. T. - "When it goes on and on and you go through endless craziness and disappointments, how can you go through that for so long and remain sane?"

R. G. - "Disappointment with myself, with reality, with the incompetence. The intensity of downfall, the breakdowns I experienced every time I wasn't conceived".

N. A. - "Emotional vortex that I have inside, feels as if it isn't me, as if the body and the soul are fighting each other and it stresses me".

L. H. - "Dealing with all sorts of side effects that I experience in every hormonal treatment is always extreme and the soul simply gets exhausted on the way to achieve the goal".

Whereas few interviewees had positive thoughts, as they describe:

K. L. - "The hope and attempt to be optimistic and positive and with positive energies, however with maximal caution so I am not disappointed. It is different because I already have 
two children at home therefore the perception is different from people who have no children, I am more aware".

G. Y. - "I try to be positive and happy and maintain normal life, but there are occasional downfalls. Now when I have children I am in a different place, not as stressed as before". N. S. - "As long as there is a chance however small I can't give up. This is my focus in life right now".

H. A. - "Overtime I realized there are women who simply fail or who give up after years of suffering or turn to an egg donation so there are no guarantees. It is possible that the journey would end without a child and I will have to decide when I quit".

\subsection{Emotions}

Fertility treatments naturally awaken a renewed hope to conceive and succeed, however they are as well demanding and hard, damaging and interfering with the life routine. As a result, the patients might feel high and unfamiliar levels of stress, anxiety, anger, damage to selfimage and in the daily functioning. They often experience an emotional roller coaster spanning between optimism and hope and between depression and disappointment. As they describe:

L. Z. - "I feel I am being tossed from one pole to the other, after a treatment there is hope and then you are happy and optimistic, as the days go by when the Beta date is approaching you start already to be stressed and nervous and after the negative answer the disappointment is great, pain and crying and then all over again - happy, stressed and nervous, sad".

G. Y. - "When the period comes there is great disappointment and then in the beginning of the treatment there is again a full month of expectation".

H. C. - "I cry from every possible thing including a TV program, the body becomes extremely sensitive to everything. My husband asked me why? I yell at him - I don't know". L. A. - "Sometimes a feeling of frustration, feelings of anger, sadness and happiness at the same time. I had mixed emotions".

N. S. - "Depression exchanged with a bursting laughter in seconds. I started to laugh about me crying for nothing and for about ten minutes I laughed and cried at the same time".

R. Z. - "There are swings in mood which are sometimes uncontrolled, I get easily nervous from a silly thing or suddenly cry for no reason". 
K. G. - "There is an emotional tossing between hope and disappointment and despair once again".

P. G. - "There is no optimism already and you no longer believe it happens to everyone at the end, right now I feel nothing but nerves when girls complain about their children and how difficult it is for them".

H. A. - "There is high emotional price, I felt like a roller coaster moving between hope and disappointment and which contains strong emotional downfalls".

H. T. - "Sometimes it felt like a crazy roller coaster, the extreme transition between despair and hope is exhausting".

R. G. - "The treatments take a lot of emotional resources, hopes shattering again and again with arrival of the period."

N. A. - "When I received another negative Beta for the collection, I burst into crazy crying, an outburst of frustration and despair".

Whereas three interviewees reported on positive feelings, as they tell:

K. L. - "There is a difficulty, it breaks and shatters inside again every time when the answer is negative, however the easiest thing is to break down and give up".

S. B. - "In every treatment there is a renewed excitement eventually proven false. Overtime, each failure strengthened me".

L. H. - "It hurts to get another negative, sometimes I feel broken and finished however I let myself fall and despair but only for a moment".

\subsection{Patient's actions}

Sometimes the process of treatments becomes a thing that is unstoppable and the patients find themselves in a repeating cycle of treatments and continue treatment after treatment without end with the thought that as you fail more, you have more confidence regarding success next time. Opposite to them, there are patients who are exhausted and choose to take a break and only one patient decided to completely give up treatments. As they describe:

K. L. - "We decided that if it won't succeed in 3 treatment cycles I will stop and reconcile with what I have today and that makes the process more bearable".

G. Y. - "In the meantime, I still continue, I don't lose hope". 
R. Z. - "I will not stop until I 1 know I did everything in order to realize my motherhood despite the financial, mental, emotional and physical difficulty and despite of being alone in this".

K. G. - "I don't think about taking a break until I am conceived, I will keep on fighting".

L. A. - "I want to continue with the treatments, however my husband suggested we take a break. Meantime I hesitate".

S. B. - "At some point the treatments become sort of a routine and there is no choice but get used to it, to keep on and believe".

H. T. - "I am going on with the thought that a child will close our family circle".

L. H. - "I continue on the path and say that maybe this time this is the right timing and there is a chance I will succeed and maybe next time it will happen".

Five interviewees have chosen to take a break, as they describe:

L. Z. - "Indeed, I was into the treatments and it is a sort of addiction, because there is a dynamic of going on and on until it succeeds and I had difficulty to stop. At some point, it caused us to argue and fight and we almost got divorced because of that, so I decided to take a break in order for us to be able to recover".

H. C. - "The changes in mood, hoping again and get disappointed again is hard for me. My head does not work for a few months, it is so hard I don't function. I decided to take a few months' break".

N. S. - "I feel that I have to take a break of a few months, I had a miscarriage of tweens in the $10^{\text {th }}$ week".

P. G. - "I decided to take a break for a month because I felt that my body is already exhausted from the treatments".

R. G. - "We decided to take a break in the treatments for a month, the side effects of hormones are hard to me".

N. A. - "The disappointing results sometimes lower the mental strength required for success, therefore it is very important to take a break every once in a while, in order to clear the head and organize the thoughts".

Whereas H. A. decided to give up as she told: "After going through many disappointments and countless negative Betas I decided to give up this longed-for dream. Right now this is the 
situation and that is my feeling, maybe it will change in the future but right now I give up, just like that".

In summary: following the hormonal treatments there are occasional rises in the nervousness, and the tendency for sadness and crying, while these changes in moods occasionally cause not only distress but also damage to relations with spouses, colleagues, friends, family members and the environment, especially when duration of the treatments prolongs. 


\section{Chapter 5: Discussion}

For a long time, fertility treatments are a subject of ongoing discussion on when, where, why and who should use them. A significant part of this argument examined the use of ART (Assisted Reproductive Technologies) in order to form families that exceed the traditional model of a heterosexual couple and their children. (Nazem et al, 2013). The topic of fertility and birth in Israel is of great social and cultural importance, many studies show that in the Israeli society birth rates and marriage rates are higher compared to other countries (VinerUman, 2012). Another indication is that Israel is the leading country in the world per capita, According to data from the Ministry of Health In 2016 there were 41,143 IVF cycles. the number of in vitro fertilizations is also high relative to the size of the population. The current study was designed to understand the emotions and coping strategies of women undergoing fertility treatments. The research population consisted of fertility patients from 3 different family structures: Married women, single women and with female spouses (lesbians). The research questions have examined whether the family structure has an impact on women's coping with fertility treatments, Is the dyad support system for women fertility treatments necessarily more stable compared to that of single women or lesbians? Does the type of treatment influence women's cope with fertility treatments? Do women with children cope differently than women without children? And Does the age of the patient have an impact on her coping with fertility treatments? The research tools were questionnaires and personal interviews. The study was an integrated study which Quantitative and qualitative methods were used.

The quantitative study included the following findings:

\section{Family structure}

The first hypothesis was that the patient's family structure would have an effect on her coping with fertility treatments. The findings show that a significant correlation was found between marital status of the patient in a way she copes with the treatments. The correlation was examined in several parameters, when the first parameter examined Actions during trying to get pregnant by marital status, it was found that although most women consulted fertility specialists, rates were different by marital status. Higher percentage of women with female partner contacted fertility specialists, in comparison to single and even lower percentage among married women. possible explanation to the differences can arise from the central role attributed to parenting in most cultures in general and in Israel in particular, luck of success to 
achieve pregnancy entails a social stigma and threatens the self-esteem of the women and men due to their inability to fulfill the perceived role. Sometimes it is not easy to acknowledge the fact that one of the spouses has a fertility problem and thus, feeling of embarrassment prevents them from seeking professional help and they tend to wait for a while. Although reproduction traditionally has been regarded as an aspect of marriage, single persons and lesbians also have interests in having and rearing offspring even if they are not married or partnered to persons of the opposite sex. To do so they turn to a fertility doctor as their only way to fulfil their dream of parenthood (Kaplan, 2012).

Additional parameter examined the correlation between the patient's marital status and the accompanied treatments in attempting to conceive. It was found that most single women underwent more treatments compared to married women and lesbians and a high percentage of these women have performed IVF treatments- these treatments range from ovulationinducing fertility drugs to invasive procedures such as Intrauterine Insemination (IUI) and Assisted Reproduction Technology (ART). it was found in the literature that single women who seek fertility treatments are usually older women in their late 30s who choose to become single mothers due to the ticking biological clock, the desire to fulfill motherhood and the understanding that the awaited man will not arrive. Studies supporting the findings of this study like Hashilini-dolev's study (2013) it was mentioned that female fertility is age dependent, meaning that fertility decline begins in their early 30 s and increases significantly in their late 30s. the profound effects of women's age on fertility are reflected in the results of advanced fertility treatments such as ovarian stimulation with gonadotropins and IVF. Older women are less responsive to gonadotropins and produce a lower number of follicles despite higher gonadotropin doses and therefore require a greater number of treatments. Accordingly, Israeli policy is that women ages 39 years and older can be offered access to IVF as initial therapy. Gliecher et al (2019) indicated that many single women mainly in their 40s seek fertility treatments to conceive. Since natural fertility declines after this age, IVF treatments in these women arise significant medical, moral, and social ethical issues. These women have a decrease in egg yield and egg quality. Another study supporting the study's findings is an article by Armstrong \& Akande (2013) which states that contemporary literature supports that IVF is the best chance for older women to become pregnant and that choosing an ovulation induction and IUI can waste valuable time and reducing the chance of success with IVF. In this context, another parameter that examined years of fertility treatments by marital status revealed that while most women in the study tried to conceive through fertility treatments 1-2 
years, $20 \%$ of single women tried to conceive even longer, 2-3 years. since fertility treatments cannot compensate for the decline in natural fertility and poor ovary reserve that occurs with delayed childbearing (Liu \& Case, 2011).

While in Hourvitz's study (2009), it has been argued that biological ovarian aging can occur independently of chronological age and today as opposed to the past we encounter with reduced ovarian reserve even in younger women, approximately $10 \%$ of women will have decreased ovarian function in their early to mid-30s. It has been suggested that a critical number of follicles rather than a critical age will determine menopause.

Examining testing following attempt to get pregnant by marital status, it was found that while higher percentage of married women conducted PAP tests, lower percentage was found among women with female spouse. There is a myth that lesbian women do not need PAP smears because they do not have sex with men, However recent data strongly supports that transmission of sexual disease can be passed on between women, even if neither of them has ever had sexual contact with a man. Moreover, some lesbian women had heterosexual relationships in the past and despite these fact and the recent findings, luck of awareness among lesbians as well as health professionals regarding the possibility of STD and hence do not refer them for this test nor emphasize its importance (Curmi et al, 2014).

\section{The impact of infertility on spousal relationships}

The second research hypothesis was that the support system for married women is more stable compared to that of lesbians and single women. The findings show that while married women reported more emotional support from the family compared to lesbians and single women, women with a female spouse reported higher partner's support and greater involvement following reproductive treatments compared to married women. That is, the hypothesis was not confirmed. In general, it seems that aspects that influence heterosexual relationships have the same influence upon same-sex couple, however, Studies have shown that the stress of parenthood negatively affects relationship's quality and radiates marital happiness among heterosexual couples. Studies support the research findings as the study by Tao et al (2012) indicated that fertility is related to marital problems and conflicts in relationships and has serious consequences on the social and mental well-being of those involved. This can be problematic since the couple's relationship is seen as the most important source of support in the context of fertility treatments. Peterson et al (2009) also indicated that when spouses experience infertility, they encounter changes in their family, social, and personal relationships. The cumulative weight of these unexpected changes can produce fertility-related stress in one 
or both spouses and how the couple responds to these pressures has implications for both spouses. Faria et al. (2012) also indicated that in most cases, infertility has great impact on emotional condition of the spouses, which creates emotional disturbances of an individual or on the intimacy and might contribute to development and/or worsening of problems in married life.

Despite the higher partner's support and involvement during treatments among same-sex couples compared to that of the heterosexual couples, results also showed that married women reported higher relationship satisfaction following reproductive treatments. In contrast to the study's findings, study by Borneskog et al (2014) that examined the quality of relationships among lesbian and heterosexual couples undergoing reproductive therapy found that lesbian couples reported stable relationships and high satisfaction with their relationship even after unsuccessful treatment compared to the decline in relationship quality among heterosexual couples. It has been suggested that same-sex couples may be more effective than their heterosexual counterparts in their ability to navigate conflicts and work harmoniously on shared tasks. Some suggest that women are better support providers than men and female spouses provide better support, which may explain the low level of conflict among lesbian couples. another suggestion explaining the decline in relationship satisfaction might be an expression due to a pressure by social expectations to form a traditional nuclear family with children.

According to Getz (2012), women experiencing infertility can often feel trauma, craziness, and loneliness, however, men often tend to ignore upon a discussion of feelings regarding infertility. Men are often bothered by the fact that they are infertile and thus disappointing their family

The most common coping strategy of both couples in the sample following failure of reproductive treatments are: Shared recreation, nevertheless, it is interesting to note that among married women $12.6 \%$ reported that both women and their spouses faced their feelings in different ways (their own way). Many studies attempted to explain the psychopathological mechanisms of psychological distress in infertile couples, they assumed that coping and attachment strategies are different according to gender, as there is difference in infertility perception between women and men. Women perceive their infertility as a threat and loss more than men, which intensifies their distress (El-Kissi, 2013). As indicate by O'Donnell (2009)- men indicate that they can overcome their feelings on their own. Furthermore, couples can report on a feeling of threat to the relationship occurring simultaneously. As infertility can be quite a stressing experience, couples usually make various behavioral or emotional efforts 
to manage, change or regain control over their lives. the gender difference might allegedly be the extent of emotional support each of the spouses can offer to the other. Men respond to infertility in the same way as they do in respect to other problems, on the other hand, women perceive infertility as a different problem of life and some even compare it to cancer. It has been argued that the effect if infertility on women is more "direct" as opposed to men who experience with infertility is "indirect" through their relationships with their spouses (Ying et al, 2015).

Feelings following attempts to get pregnant by marital status revealed that the most dominant feeling reported by women in this study following attempts to get pregnant is frustration, In addition, women reported strong feelings of stress, pain and disappointment whereas, the least experienced feelings are hope, sadness , and loneliness. Although infertility is not a disease, the treatment of this condition requires medical interventions which might put women at a risk of depression symptoms especially after failure of a treatment and the emotional effects can be like those of a severe chronic disease. Fertility treatments that offer more and more people a possibility of becoming parents are involved in complex mental coping: they are demanding and difficult, complex, and cumbersome for execution, damaging for self-image, spouse relations and the fabric of life. This crisis is called in literature - Reproductive Trauma (Kapln, 2015). When fertility treatments are proceedings for a long period of time, administration of intricate medical drugs according a predetermined program - all these add to the pressure of diagnosis. Psychological influence of hormones women intake during fertility treatments were have not been thoroughly researched, even though fluctuations in mood and depression are known as side effects of ovulation induction medical drugs and that women undergoing ovulation induction often complain about psychological problems. most common consequences include turmoil, frustration, depression, anxiety, guilt, hopelessness, and feelings of worthlessness in life (Lake, 2012). As compared to infertile, men, barren women express higher distress regarding infertility and tend to initiate more in a pursuit after treatment possibilities. These data indicate that women are more willing to undergo the required treatment for achievement of parenthood (Lopes et al., 2013).

It was also found that about half of total sample reported they did not need any mental assistance while almost all women with female partner did not need mental assistance in comparison with married women and single women. The majority of female spouse shared their feelings with their spouse in comparison with their heterosexual counterparts. A study by Ginor 2015 who examined the emotional effects of infertility and fertility treatment among 
married couples revealed that heterosexual couples are both in a state of emotional need and therefore find it difficult to be attentive to each other and support each other and it is often possible to see reactions of interiorization and anger towards a spouse . however, like women, men suffer from coping with fertility treatments both physically and psychologically. While there are many evidences of emotional adjustment of women to infertility, there are no systematic studies focusing on psychological adjustment of men to infertility and the treatments accompanying it and most of the studies regarding psychological aspects of infertility focused only on effect of infertility amongst women (Mikkelson et al., 2012).

In contrast, Chachamovich's (2009) study of quality of life among men and women suffering from infertility that does not support the study findings suggested that infertility may be stressful, but the couple's shared status brings close mutual support for their thoughts and feelings and thus has a positive effect on their relationships.

\section{Family's support}

Among all women, encouragement and empathy were rated as the most common type of emotional family's support while married women gained the highest rate of empathy. This finding can be supported by the fact that In Israel the traditional family structure remains dominant, parenthood is viewed positively, and heterosexual marriage is considered the most legitimate framework for having children (Shechner, 2013). therefore, lesbian as well as single mothers by choice belong to marginalized minority groups in the society. Childbearing outside of the traditional family model is frequently greeted with ambivalence of family and friends and insufficient support (Ben-Daniel et al., 2007). some families exhibit disappointment because the woman will not be fulfilling their dream and giving birth within the traditional marital system (Nevo,2015). On the other hand, about half of sample reported they had no emotional support from close family with higher rate among women with female partner. The emotional impact of infertility creates problems not only to spouses, but rather to their family members and friends as well, and therefore social support can be a critical component of the way a woman adapts to the unexpected stress of infertility, especially as most women expose their infertility to others, and to a larger extent than men. Many people want to be useful and supportive, however they do not know how. Despite their good intentions and their efforts, they usually say and do the wrong thing, whether hurting a person or hurting themselves. One of the main reasons for family and friends to find it difficult to help is that they know so little about the emotional aspects of infertility, starting with the shocking diagnosis and the demanding treatment to disrupting daily experiences. This 
emotional overload can cause one to become depressed, angry and filled with guilt, and family and friends often add to the pain with good intentions nondeliberate remarks and actions (Mahlstedt, 2009).

Appreciated familial support can influence the way a woman experiences stress of infertility both directly and indirectly. Findings of the study insinuate that perceived support from a family might assume a more direct role in relieving the stress of social infertility. perceptions regarding the support a family provides not only reduce social problems related to infertility, but as well are indirectly useful in four fields of infertility stress (worrying regarding relations, sexual worry, rejection due to lifestyle without children and need for parenthood) (Martins et al., 2014).

\section{Coping strategies by marital status}

Surprisingly, among all women, the most common coping strategy to failure in reproductive treatment is being optimistic, however, women also report they cry and get sad, while married and single women cry the most. In Adler's viewpoint, when a person/couple faces fertility problems, they face challenges in all five fields of Adler's life missions which are: self, love/sexuality, community, work, and spirituality. when one runs into low satisfaction in missions of life, he/she is expected to show signs of depression, anxiety, and sense of low selfesteem (Siedlecki, 2013). Considering the traumatic nature of infertility, as well as the impact it has upon the five life missions of Man, it seems that there is a direct impact upon the mental health of a person, men and women struggling with infertility, find themselves dealing with multiple challenges. All those challenges might bring about an excessive chronic pressure that might lead to anxiety disorders as well as depression (Tompson, 2013).

As infertility is a stressful factor with low control, women can do truly little and even nothing in order to actively change the nature of the situation. As a result, passive coping styles and emotion-focused strategies which include efforts to focus on something else than the stress source and relieve the anxiety can also be suitable. Optimism was found as a problem-focused coping tactic. In general, problem-focused coping is the best, as it removes the pressure, deals with the root of a problem, and supplies a long-term solution. However, it is not always possible to employ problem-focused strategy, for example in a case when sense of loss accompanies fertility patients, those strategies will not be particularly useful, as coping with the sense of loss requires emotion-focused coping. Moreover, problem-focused approach will not work in any situation where remove the stress is beyond one's control. They operate better when one can control the source of stress .while coping strategies themselves can influence 
directly the stress related to infertility, they can as well be used as mediator variables between social support and stress (Martins et al., 2011).

It was found that after IVF treatment failure, women tend more to use the following coping strategies: confrontation (meaning, women wanted to find out more details about the problem and then to learn something new in order to cope with the problem), optimism (meaning, women tried to think positively and hoped the situation would improve) and relying on oneself (meaning, women kept their emotions to themselves and wanted to be alone in order to think things through) as efficient coping strategies (Lee et al., 2014).

Differences in effects of treatments by marital status also were examined, it was found that social encounters affected most of the women in the sample during their treatments with a slightly higher tendency in single women. while fertility treatments realize the yearning for motherhood, they as well emphasize an inability of a woman to become a mother. Research literature regarding this impact indicates that the feeling of "damaged identity" following fertility problems causes stress, social isolation, frustration, and conflict with the environment. women who suffer from infertility probably tend to alienate from social events with presence of little children. All of these can cause strong emotional reactions, meeting friends who have children is more difficult and it contains feelings of jealousy and anger about the situation created, Added to the inner psychic experience is also the social stigma associated with the absence of children, especially at the ages of relatively older ages. This may in some cases lead to confinement at home and avoidance of going out to social gatherings, hiding the situation, or even attempting to misrepresent the reluctance of children (Haelyon ,2010). Infertility might cause couples and individuals to withdraw and feel detachment from their community, their friends, and social events and thus they become more and more isolated, which might lead to feelings of depression. According to Getz (2012), women, such as single women by choice, who have experienced lack of empathy and support and lack of sensitivity on the part of close friend, might brought about breaking of multiple friendships.

It was also found that the daily routine of single women is slightly more affected than for married women and women with female partner. during fertility treatments, especially require performing of hormonal tests and Ultrasound which being performed every few days, depending on the treatment plan, response to treatment and the instructions of the attending physician between tests. frequent visits for tests and procedures accompanied can cause changes in the current lifestyle end even disrupt it. fertility treatments had repercussions on various areas of life, such as marital relations and social relations, sense of well-being, general 
quality of life and workplace behavior. this often involves absence from work. Women often do not share with their boss they are undergoing fertility treatment, they are forced to justify their absence which causes friction in their workplace (Mookim et al, 2010).

single women believe that it was a matter of fate while married women and women with a female partner have accepted the situation somewhat more than single women. fertility treatments had repercussions on various areas of life, such as marital relations and social relations, sense of well-being, general quality of life and workplace behavior. According to Richard Lazarus (1984) cognitive appraisal theory accepting the situation is Secondary appraisal which involved in emotions like those related to coping with stress factor or with the stress it manufactures. Statements such as: "I can do it if I do my best", "I will try whether or not my success chances are high", and "if I fail in this way, I can always try another approach" - implies a positive secondary appraisal. Opposite to that, statements like: "I cannot do it", "I know I will fail", "I will not do it since no one believes I can" and "I will not try as my chances are low" - implies a negative secondary appraisal. Harpaz indicates in her paper from 2010 that processes of secondary appraisal are influenced by intensity of threat, factors comprising the stress situation, the psychological structure of a person and coping resources at his disposal.

\section{The emotional effects of treatment, especially of IVF}

The third study hypothesis was that IVF treatment was positively correlated with coping. The findings support the hypothesis and show that women undergoing IVF treatments were significantly more emotionally affected than women undergoing medical therapy and IUI. Moreover, among women who conducted IVF, they mostly did not overcome the crisis. Although the literature notes that many women who undergo fertility treatments experience significant distress, IVF is an alternative treatment after failure of fertility treatments. Yaun's study (2013) indicated that IVF treatment is psychologically and emotionally stressful. The stress before, during and / or after IVF treatment is multidimensional. There is the source of chronic stress caused by the threat of permanent infertility and loss of hope. Another source of stress is the threat of treatment itself such as oocyte retrieval and the possibility of failure in each of the different stages of treatment. Oocyte retrieval and waiting period for pregnancy test found the most stressful stages in IVF cycle. Malina \& Pooley (2017) also noted in their study that IVF is a time-consuming and invasive treatment. Despite the relatively low chance to achieve pregnancy in one IVF cycle, many women entering treatment have unrealistic 
expectations about the treatment's success. IVF for all its painful and invasive procedures is an oppressive treatment. Since IVF is sometimes the last treatment option in terms of fertility treatments, patients need to face the possibility that they will never achieve the goal of life's important parenting. As a result, IVF treatment poses a threat to a person without children who may cause symptoms of anxiety. Furthermore, IVF is a low control pressure factor since patients have little control over its progression and outcomes. As the treatment progresses and its uncontrollability continues, feelings of depression may emerge. Domar's (2016) study also indicated that many IVF patients report symptoms of depression even before starting their treatment cycle, which probably reflects the consequences of recurrent invasive treatments repeated incessantly. Most IVF patients state that the treatment is more stressful psychologically than physically, and the main reason why patients discontinue treatment is psychological burden of the IVF process rather than financial burden as it used to be. A systematic review of 27 studies focusing on women's emotional adjustment to various stages of IVF treatment was conducted in 2007. The survey examined symptoms of mood and anxiety in women undergoing IVF treatments / embryo transfer found a moderate rise in depression and anxiety indexes was observed during entire treatment cycle.

However, findings also revealed that level of faith or acceptance of the situation were not different between women according to type of treatment. maybe one explanation for this can be found in the fact that the Israeli society attributes heroism to mothers of all ages, at any price, any health condition, and any financial, family or emotional condition, it is therefore hard to resist the track of grueling treatments. The only track that wins the support and social legitimacy is the state of fertility treatments and therefore couples and individuals make significant efforts to bring a child into the world, even though the treatments are expensive, exhausting and dangerous and at later ages are of lower odds of conceiving (Hashiloni-Dolev, 2013).

Studies reveal that the main sources of pressure of fertility patients are impact of infertility upon their social lives, their social health, and their relationship with their spouses. Stress level in fertility patients tends to grow as the treatment is more intensive and as the duration of treatment prolongs. Therefore, IVF patients are expected to experience more pressure than patients who are in early stages of their infertility assessment. Additionally, medical drugs that are employed for treating infertility might contribute to depression symptoms (Domar, 2015).

An article by Molgora et al (2019) indicated that some studies reported that the emotional distress experienced by fertility patients does not depend on the ART technique (type of care or number of previous treatments). However, according to some of the authors stress can 
affect patients' decision to discontinue treatment. In most cases, patients arrive at IVF after unsuccessful attempts to conceive with conventional fertility treatments and a sense of failure that accompanies any unsuccessful experience, in a never-ending series of unpleasant treatments, tests and feeling that nothing is progressing, for which IVF treatment is their last resort and hope to conceive.

\section{Secondary infertility}

The fourth research hypothesis was that coping was negatively correlated if the couple already has children. It was found that women with no children are affected more negatively than women with children and did not overcome the crisis. According to the WHO definition, when a woman is unable to bear a child, either due to the inability to become pregnant or the inability to carry a pregnancy to a live birth following either a previous pregnancy or a previous ability to carry a pregnancy to a live birth is called Secondary Infertility. While the prevalence of infertility in the US is approximately $15 \%$ to $17 \%$, Secondary infertility may comprise $80 \%$ of these cases, though reports vary. Many people assume primary infertility is more common than that of secondary infertility, but according to a review published in 2018, secondary infertility is the most common form of female infertility. Couples experiencing secondary infertility may be more likely to put off seeking help. They may also find that friends, family, and even doctors decry their fertility struggles because they have one kid or even more, it should be enough. Secondary infertility is caused by the same problems that lead to primary infertility. Some couple think that the fact that succeeded to conceive in the past guarantees they will conceive again on their own. sadly, there are no fertility guarantees, for anyone (Benksim et al, 2018).

In a 2011 study by Bradow, much of the research on infertility and the psychological effects of diagnosis and its treatment focus on primary infertility. Experiences of women and couples coping with secondary infertility has been neglected both from research aspect and the possible difference of the psychological consequences. Naturally, women without children who wish to conceive experience greater mental distress due to fertility problems than women who have already given birth (or adopted) children, since women that have children have already fulfilled at least part of their desire to parent, nevertheless, Secondary infertility can be just as devastating as primary infertility. It is important to recognize that it is important for people not only to form a family, but to have the family size they want. Secondary infertility treatment is usually the same as that of primary infertility. White \& McQuillan (2006) assumed that since couples or individuals with secondary infertility have already a biological 
child they do not suffer as much as those who suffer from primary infertility when deciding to stop trying to conceive, However, they did not find a significant difference in distress between primary and secondary infertility. This study only assessed the possible difference between the distress when couples decided to stop trying to conceive and not what the couples experienced during diagnosis and treatment. what seems to be missing in the literature is an assessment of the experiences of couples who already have children during the diagnosis and treatment of secondary infertility. In the literature reviewed, the studies appear to focus solely on primary infertility, or do not differentiate between primary and secondary infertility.

It was also found that women with no children believe that difficulties in getting pregnant is a matter of faith more than women with children. Gameiro (2015) also pointed out that patients who find it difficult to accept their infertility and have no children are more likely to experience anxiety and depression when being notified that treatment has failed.

Additional finding examined differences between women with and without children in coping strategies yield similar results among the two groups. both women they mostly did not overcome the crisis with slightly higher tendency in women who have no children, nonetheless, many reported they were optimistic with slightly higher tendency in women with children. Childlessness was found to result in perceived role failure, with social and emotional consequences for both men and women, and often resulted in social stigmatization of the couple and particularly in women. Infertility places women at risk of social and familial displacement, and women clearly bear the greatest burden of infertility

\section{The connection between a woman's age and coping}

The fifth research hypothesis was that women's age is positively associated with motivation to deal with fertility treatments. Results showed that younger women have more support, but they are also more negatively affected by fertility treatments compared to older women. The literature found two articles discussing the difficulties associated with fertility treatments among younger compared to older women, but no articles were found to discuss differences in coping among younger women compared to older women.

In a study by Marino (2010) conducted in Australia examined fertility treatments and young women using them. It was found that nearly a quarter of women in their early 30 s reported difficulty to conceive and more than a quarter reported a loss of pregnancy. This implies that quite a few young women have significant difficulties in trying to conceive. 
While social changes have led to women delaying their childbearing, advances in reproductive techniques have led to increase of options for fertility treatment and ART. Unfortunately, this may give women false optimism that they can pursue their careers and try waiting for their right man with the expectation that ART will help them to conceive if they have difficulty conceiving later. Although chance of success diminishes with age, IVF still offers higher pregnancy rates and live birth rates than conventional fertility treatments, although in many cases IVF cannot compensate for delays in childbearing (Liu \& case, 2011). An article by Gourounti et al (2012) found that patient's age significantly related to fertilityrelated stress, anxiety, and depressive symptomatology. The results of the study indicated that older women had higher levels of stress, anxiety and depressive symptoms associated with infertility than younger women.

\section{As part of the qualitative research findings were as following:}

The study included 5 themes derived from interviews with the interviewee

1. Attempts to conceive and the attendant difficulties- The first theme said that most people believe that when they decide to become pregnant that will happen shortly, but some find to their disappointment that in many cases it is not an easy and fast process, sometimes it takes quite a while for it to occur and sometimes accompanies this process a lot of stress and on the opposite side there is a pressure from the society, the family and the surroundings to have children. The literature talks about attempts to conceive and chances of conception in the first year. Kouhung \& Hornstein (2016) indicated that the concept of reproductive capacity has been shown to be useful for determining the proper parameters in the study of fertility potential. European prospective study investigated 346 women from the general population who were trying to conceive, it was found that the cumulative chances of conception for the total group at the end of 1, 3, 6, and 12 months were $38 \%, 68 \%, 81 \%$ and $92 \%$, respectively. The researchers estimated that after 6 months, $50 \%$ of the remaining couples were infertile or sub fertile. Anwar's (2016) article also indicated that the chance of conceiving depends on the length of sexual exposure, frequency of relationships and age of the couple. Only $5 \%$ of couples conceive after a year and a half or two years.

For lesbians and single women, attempts to conceive are expressed through attempts to conceive through the various fertility treatments. The interviews show that patients assume 
that they will have no problem conceiving because they are "fertile" and have not experienced problems in the past.

2. Response to finding out about the problem - searching solution said that after a year of unsuccessful attempts to conceive, appeal to fertility specialist to assess fertility status. In some patients the problem is revealed in them, some with spouse and in some there has been no problem found and clarification continues. Knowing that there is a fertility problem causes severe reactions of shock, mourning, denial, anger, repression or emotional outburst, sadness, and depression. Occasionally, fear arises from discussing the issue with family, friends, workplace or professionals. The literature describes infertility as a global problem affecting people around the world whose cause and importance may vary depending on the location and socioeconomic status. Deyhoul et al (2017) indicated that 10-15\% of couples worldwide suffer from infertility problems when about half of the cases, infertility is attributed to men. Estimates show that $30-45 \%$ of the men are infertile, in $30-45 \%$ of cases the woman is infertile and in $20-30 \%$ of the cases are related to a combination of other factors. A wealth of studies has demonstrated the physical, psychological, and emotional impact of infertility. Hocaoglu's (2018) study found that infertility causes personal, family, and social problems and in fact this is a devastating health issue since infertile couples or individuals often exhibit stress, anxiety, depression, feelings of guilt, fear, loss of social status and despair. However, a significant number of infertile couples or individuals choose to hide the problem and avoid sharing the fertility problem with their families and their relatives, which leaves them no support. In the study by Deka-Kumar \& Sarme (2010) it was found that the stress of not fulfilling the desire for a child is associated with emotional turmoil such as anger, depression, anxiety, relationship problems and feelings of worthlessness. Rooney \& Domar's (2018) study indicates that infertility is a life crisis affecting patients worldwide. Infertility patients experience a huge amount of emotional turmoil as a result of their diagnosis, the risk of depression, anxiety and distress is high for these patients. Since Biblical times, it has been hypothesized that stress may impair fertility. This raises one of the most fascinating questions: Does infertility cause stress or does stress cause infertility? The answer so far is unclear. the relationship between stress and infertility may not have a clear cause and effect. A European study found that anxious women took longer to conceive and were more likely to have an abortion than women with lower anxiety levels.

While heterosexual couples could get the diagnosis of infertility after one year of unprotected intercourse, lesbians and single mothers by contrast could be diagnosed after several failed 
rounds of IUI cycles. In a paper by Campo-Englestein \& Lo "expanding the clinical definition of infertility, so that social infertile individuals will be included as well as couples", they suggested that infertility diagnosis should be available to anyone who during a twelve months period hold the willing to conceive but cannot due to social or physiological limitations. meanwhile, the statistics about infertility is based on the reports of married heterosexual couples ignoring the single or lesbians women who cannot have children for reasons that are sometimes related to their bodies.

3. Choosing an appropriate solution and beginning treatment - The third theme said that fertility treatments are given according to the problem that has arisen from the various tests and in cases where the problem is not clear the treatment is carried out by trial and error. Regardless the source of the problem, the woman has to undergo the treatments. waiting for the results of the treatment is often difficult and nerve-wracking and knowing that the treatment fails brings a sense of despair and frustration. Kuohung \& Hornstein's (2016) study also indicated that after identifying the cause of infertility, treatment aimed at correcting reversible factors and overcoming irreversible factors can be given. In addition, the couple advises lifestyle changes to improve fertility such as quitting smoking, lowering caffeine intake, and appropriate timing and frequency of intercourse. therapeutic interventions for treating infertility of men and women can be involving medication, surgery and / or procedures such as artificial insemination or IVF. A wealth of articles have been written on the emotional effects of fertility treatments, in Nahrin et al's study (2017) it is noted that women (more than men) almost always experience a greater degree of emotional distress due to infertility, she may feel embarrassed each month that the pregnancy attempt fails. treatment of infertility is known as "emotional roller coaster" because of the ups and downs associated with the process - for every month when pregnancy does not occur, many hours are filled with hope followed by disappointment or, at best, despair.

4. Coping with the outcomes of the chosen treatment - the forth theme said that fertility treatments could end in failure thus causing disappointment and frustration or pregnancy which indicates the success of the treatment and causes joy and happiness, but sometimes pregnancy ends in miscarriage in the early stages or later what causes aggravation and difficulty coping. There is a significant debate in the literature there is significant debate regarding the number of treatment cycles couples should perform. The article by Smith et al (2011) indicated that transition to IVF after 3 failed IUI cycles resulted in faster pregnancy than performing 6 IUI cycles before IVF. Data from the Society for Assisted Reproductive 
Technology (SART) showed that cumulative pregnancy rates in fertility treatments stabilized after 3-4 cycles of treatment. However, the pregnancy rate for each treatment cycle slowly decreased from a maximum of $30 \%$ for couples undergoing one treatment cycle to about $20 \%$ for couples undergoing 6 or more cycles.

brandes et al (2011) is indicated that while the average incidence of clinical abortion varies between $10 \%$ and $15 \%$, abortion rates after fertility treatments range from $18 \%$ to $30 \%$. The mechanism that stands at the base of this increased risk is not entirely clear, in general, important risk factors identified to miscarriage are women's age, previous pregnancy loss, obesity and smoking. Others researches like Yang et al (2016) found that after adjusting risk factors such as women's age and recurrent miscarriages, pregnancies after treatments have higher rates of miscarriage than natural pregnancies among infertile couples. In the research of Rafaat et al (2015) it was found that a history of infertility and fertility treatments increases the likelihood of an ectopic pregnancy and prevalence higher than 2-3 times than in the general population. Fertility treatments can also result in heterotopic pregnancy which is an ectopic pregnancy along with intrauterine pregnancy. spontaneous heterotopic pregnancy is extremely rare (1.30000 pregnancies) and its incidence has increased due to various fertility treatments. After IVF prevalence ranges from 1-3\% of all clinical pregnancies.

However, Pezeshki et al (2000) found that infertile women who conceived after fertility treatments did not show an increased risk of miscarriage compared to women who conceived spontaneously.

5. Being in treatment - The fifth theme talked about the fact that prolonged fertility treatments cause despair, become the center of life, thus damaging the existing routine of life, which causes weakening of significant social and interpersonal relationships. Patients become motivated with "one-way tunnel thinking". infertility is described in the literature as affecting women's mental health from physical, emotional, sexual, spiritual and economic aspects. Ezzell et al (2016) mentioned that the more demanding and invasive medical treatments are, so the reported symptoms of anxiety and depression are higher. Each treatment cycle that passes brings with it a rollercoaster rides of emotions such as anger, betrayal, guilt, sadness and even hope. With every friend announcing being pregnant and every pregnant woman passing by the street, the anxiety and stress of patients intensifies. According to an article by Kaplan (2012) Fertility treatments can aggravate the intensity of stress and difficulty. Research shows that emotional difficulty reaches a peak between the second- and third year of fertility treatments process. each month pregnancy is measured again and turns the waiting 
process a track of ups and downs, the stressful waiting and anticipation that the period will not appear, while a great disappointment when it comes again and again. It is also said that men may feel helpless trying to comfort their spouse and feel frustrated that they cannot solve their fertility problems. Sometimes the couple is grappling with the issue of when to "give up" and stop medical treatment after failing. Sometimes there is a discrepancy between the couple as for their desire to quit or continue and may cause accusations and anger between them.

\section{$\underline{\text { Collecting of findings }}$}

Both in questionnaires and interviews it was found that:

Women with female spouses reported higher support from their spouse in comparison to married women. In addition, among married women it was found that $12.6 \%$ reported that each spouse copes with the situation in its own way.

Women who have no children are more affected than women with children, despite the treatment's difficulties, since women who already have children are less stressed and its being a source of comfort.

Most of the women expressed frustration, pain, and disappointment when the treatments were failed, but despite the difficulty they tried to maintain optimism.

Women undergoing IVF treatment were more emotionally affected than women who treated with hormone therapy and IUI.

It was also found that when the fertility problem was revealed, reactions of most women were: shock, anger, denial, emotional outburst, sadness, and depression. Some women thought they would have no problem conceiving.

\section{Additional themes findings}

The patient's support systems who are: the society, family, spouse, friends, workplace and medical system can be a source of support for them, but on the other hand can also be a source of stress and sometimes lack of support for them. Fertility treatments are demanding, difficult, abusive, and interfering with life routine. patients experience high levels of stress, anxiety, anger, and extreme mood swings which can also affect relationships with their spouse, colleagues, friends, and family especially when the duration of treatment is prolonged. 


\section{Additional questionnaire findings}

The family structure affects women's coping with fertility treatments and is measured by several categories (concomitant treatments for attempts to conceive, referral to fertility specialist, related tests and duration attempts to conceive). It was also found that half of the sample did not receive any mental assistance while women with a female spouse reported they had no need for assistance compared to married women and single women.

Young women received more support but were also negatively affected by fertility treatments compared to older women.

Additional findings in the interviews may be since qualitative research is characterized by great flexibility and therefore there may be additional variations and possibilities. another possible explanation - most women who were interviewed felt comfortable telling in detail about their adversity, it is possible that early acquaintance with these women may have made them be more frankness.

\section{Chapter 6: Conclusions}

Most patients who seek fertility treatments are married women after failed attempts to conceive a spontaneous pregnancy, while women with female spouse and single women who seek fertility treatments, infertility is not necessarily a reason referring to treatment, but the social context which does not allow getting pregnant naturally (called in the literature- Social Infertility), the way these women conceive is through fertility treatments with sperm donation. The study showed that fertility treatments are a source of psychological distress that have devastating consequences for patients' psychological well-being. The support systems available to patients such as the society, family, friends, and even the spouse do not always know how to behave during treatments or during pregnancy loss. they do not know whether to give an encouragement, advice or comfort that can sometimes be interpreted as snooping and even hurting because of the sensitive situation in which the patients are in. Also, from the medical staff's view, despite the empathy that they express to the patients suffering, these patients sometimes have complaints that are difficult to deal with. Most patients can cope with the multiple requirements of the treatments and believe that they do not need professional support. Many patients receive referral for mental support only in a current crisis situation, which may lead to the concept of referral in the form of "punishment" as an indication that 
they need to be able to cope more positively with treatments. However, about $20 \%$ of patients experience significant distress which cost a heavy price for their coping resources.

\section{Practical recommendations}

Taking measures to prevent psychological problems that may affect treatment's success is a critical issue, not only to reduce psychological symptoms, prevent anxiety and depression, but also to improve physical health and successful pregnancy. Psychological counseling should be offered at every stage of fertility treatment and not only when treatment fails. Early preparation is needed regarding the medical procedures that couples and individuals are supposed to undergo along with the possible consequences.

It is important to identify and to bring into focus high-risk patients who exhibit significant psychological distress and refer them for professional mental assistance. Couples and individuals should be assessed in the context of psychological factors and findings as an integral part of the fertility treatment protocol.

\section{Study limitations}

Aside theoretical and practical findings and conclusions, it is important to describe the study limitations.

One of the great challenges in assessing the level of distress of women with infertility is the accuracy of self-report measures. Women may pretend to be more mentally healthy than they really are. Also, they may have heightened feelings of hope or optimism before starting fertility treatment, which is when most distress assessments are conducted.

The medications used in treating infertility include Clomifen, Letrozole, and gonadotropins, which are related to phenomena such as anxiety, depression, and irritability. Thus, in assessments of women's symptoms during treatment, it is difficult to distinguish the psychological impact of infertility from the side-effects of the drug.

Because the State of Israel funds an almost unlimited number of fertility treatments, the findings of this study may be unique to Israel. However, this potential limitation is offset by the insights gained by studying the coping strategies of women who are in fertility treatment over long periods of time.

\section{Future studies}

Current study and its findings raise several possible future research directions derived from current research limitations, from broadening the field of research to additional fields and from 
refinement and deepening of the research. Following are several recommendations for future research directions:

1. Current study examined the Jewish population. It is recommended that a similar continuation study is conducted by using a sample of additional populations such as the orthodox population as well as the Arab population in Israel.

2. Women's coping with fertility treatments should be examined according to education and socio-economic status.

3. Current study was held in Israel, which generously finances fertility treatments. Other countries with no funding and no participation in the high treatment cost should be examined.

4. Current study focused on women undergoing fertility treatments and their coping strategies, this finding opens a door to studies examining the impact of fertility treatments on male/female spouses and their coping strategies.

5. Coping with fertility treatments was examined amongst young women as opposed to older women in general. It should be examined how these women cope with the treatment according to age groups. 


\section{Chapter 7 : References}

A pregnancy diary- Information about sperm donation issues (2014). Retrieved February 3,2017, from: http// www.y-herayon.co.il

All drugs in fertility treatments. Retrieved December,11,2016 from: http://www.caremed.co.il.

American Society For Reproductive Medicine. (2012) Age and Fertility, a guide for patients. American Society For Reproduction Medicine. (2014). Polycystic Ovary Syndrome (PCOS). Fact Sheet from Reproductivefacts.org.

Anisman, A., \& Merali ,Z. (2009). Understanding stress: Characteristics and Caveats. PubMed, 23, 241-249.

Armiger, B. (1997). Ethics in nursing research: Profile, Principles, Prospective. Nursing research, 26,5,330-333.

Aulette, J.R. (2010). Defining families. Retrieved July,29,2017, from Pearson catalogue \& instructor Resources internet site: http:// www.catalouge.pearsoned.co.uk>catalog.

Bar-Sela L. (2003). A contemporary view on family; The family's revolution. In Liora B.S (Eds). Family and acknowledgement (volume 2). Ministry of education.

Benyamini y, Gozlan M, Kokia E. Women's and men's perceptions of infertility and their associations with psychological adjustment: a dyadic approach. Br. J. Health Psychol., 14 (2009), pp. 1-16, 10.1348/1359107008X279288.

Benksim A, Elkhoudri N, Addi RA, Baali A, Cherkaoui M. (2018). Difference between Primary and Secondary Infertility in Morocco: Frequencies and Associated Factors. Fertil Steril. 12(2):142-146.

Beutler, L., Booker, K., \& Peerson, S (1998). Experiential treatments: Humanistic, ClientCenterd, and Gestalt approaches. Comprehensive clinical psychology, 6, 163-181.

Bhongade, M.B., Prasad, S., Jiloha, R.C., Ray, P.C., Mohapatras, S., \& Konar, B.C. (2015). Effect of psychological stress on fertility hormones and seminal quality in male partners of infertile couples. First international Journal of Andrology, 47,336-342.

Bilney, C., \& Pillay, S. (2015). Stress and Coping during change, in eBook, Public sector organizations and cultural change (chapter 6, pp 127-141). Springer publishing. 
Bogenscheider, K., Little, O., Oomps, T., Benning, S., \& Cadigan, K. (2015). What is a family? Retrieved July,8,2017, from family impact handbook's internet site: http:// www.familyimpactsemenars.org.

Bradley, L.D. (2016). Overview of hysteroscopy (Electronic version). UpToDate.

Bunis, A.M. (2007). Families in sociological and anthropological perspective. Open university (digital addition).

Check, J., \& Schutt, R. K. (2012). Survey research. In J. Check \& R. K. Schutt (Eds.). Research methods in education. (pp. 159-185). Thousand Oaks, CA: Sage Publications. Cheng,L.Y., Lin,P.Y., Huang, F.J., Kung, F.T., Chiang,H.J., Lin,Y.J., \& Lan,K.C. (2015). Ectopic pregnancy following in vitro fertilization with embryo transfer: a single center experience during 15 years. Taiwan Journal of Obstetrics \& Gynecology, 54,541-545.

Christian, L.G. (2006). Understanding families: family dynamics. Retrieved August,10,2017, from Strong Bond Fact sheet internet site: http: // www.strongbonds.jss.org.au.>workers.

Collection of sperm by retrograde ejaculation. Retrieved March,17,2017, from: http:// medicine. Missouri.Edu/fertility.

Creswell, J. (2000). Qualitative inquiry and research design - Choosing among five traditions. London, UK: Sage.

Curmi, C, Peters, k., \& Salamonson, Y. (2014). Lesbians' attitudes and practices of cervical cancer screening: A qualitative study. BMC Women's Health, 14:153

Dai, L., \& Wang L. (2015). Review of family functioning. Open journal of social science, $3,134-141$

Domar,A.D.(2015). Psychological stress and infertility (Electronic version). UpToDate.

Domar,A.D.,Gordon, K., Garcia-Velasco, J., La Marca, A., Barreira, P., \& Beligotti, F. (2012). Understanding the perceptions of and emotional barriers to infertility treatment: a survey in four Europe countries. Human Reproduction, 27,1037-1079.

Donath,O. (2015). Pro- Natalizm and it's "cracks": Narratives of Reproduction and childfree lifestyle in Israel (Electronic version). ResearchGate publication ,11, 417-439.

Donath, O. (2011). What is a family anyway? Alternative definitions and social connections. From the "women who do not want children" forum. Retrieved from the web July,7,2017. 
http://isha.org.il > uploads>2014/09>1 > pdf.

Effroni,R.(2013). Emotional aspects of women undergo fertility treatments and IVF pregnancies. Retrieved March,20,2017, from Psychiatric service. Sorasky medical center website: http:// infant-mh.co.il.

El-kissi, Y., Ben-Romdhane,a., Haidar,S., Bannour, S., \& Ayoubi,-Idrissi,K. (2012). General psychopathology, anxiety, depression, and self-esteem in couples undergoing infertility treatment: a comparative study between men and women. European Journal of obstetrics \& Gynecology and reproductive biology, 167,185-189.

Elnashar,A.M. (2012). The possible role of natural cycle and modified natural cycle in IVF. Middle East Society Journal, 17,226-230.

Enrique,J.A., Howk,H.R.,\& Huitt, W.G.(2007). An overview of family development. Retrieved August,3,2017, from Educational Psychology Interactive's internet website: http:// www.edpsycinteractive.org/papers/family.pdf.

Families and households in Israel. (2017). Retrieved August,15,2017, from Central Bureau of Statistics Israel's internet website: http:// www.cbs-gov.il.

Fauser,CJM. B. (2016). Overview of evaluation induction (Electronic version). UpToDate.

Faria D. E. P. d, Grieco S. C, Barros SMO D. The effects of infertility on the spouses 'relationship. Revista da Escola de Enfermagem da USP.

Ferrira, M., Antunes, L., Duarte, J., \& Chaves, C. (2014). Influence of infertility and fertility adjustment on marital satisfaction. (Electronic version). Social and Behavioral Sciences, 171, 96-103.

Fertility and fecundity; The complete guide. Retrieved December 20,2016, from http:// www.poryut-guide.com.

Fertility treatments (the western and complementary aspect). (2015). Retrieved from Mavor, D. complementary and alternative medicine practitioner website: http://www.eshet$\underline{\text { marpe.com }}$

Fogeil-Bijaoui S. (2002). Families in Israel; between family and post-modern. In U. Shavit (and others) Eds. Sex, Gender, Politics 107-167. Hakibbutz Hamehud.

Folkman, S. (2013). Stress: Appraisal and coping. In M.Gellman \& J.R.Turner (Eds.) 
Encyclopedia of Behavioral medicine (pp.1-2). New-York: Springer Science \& Business media.

Frances, R., Coughlan, M. \& Crinin, P. (2009). Interviewing in qualitative research: the oneto- one interview. International Journal of therapy and Rehabilitation, 16,6.

Freedman, I. (2006). Ethics in Education research: The researcher, the interrogee and science. Research trails, 13,17-37.

Frydenburg, E. (2014). Coping research: Historical background, links with emotion, and new research directions on adaptive processes. Australian Journal of psychology, 66,82-92.

Gal, A. (2010). Infertility: When the problem is with the man. Retrieved January,27,2017, from Ynet's website: http:// www.ynet.co.il

Galliano, D., \& Pellicer, A. (2015). Potential Etiologies of Unexplained Infertility in females (Electronic version). Springer, chapter- unexplained infertility, 141-147.

Gameiro,S., Boivin, J., Dancet, E., de Klerk, C., Emery, M., Lewis-Jones, C., Thorn, P., Venden-Broek, U., Venetis, C., Verhaak, C.M., Wischmann, T., \& Vermeulen,N.(2015).

ESHRE guideline: routine psychological care in infertility and medically assisted reproduction - a guide for fertility stuff. Human reproduction,30,2476-2485.

Gates, G.J., (2015). Marriage and family: LGBT individuals and same-sex couples (Electronic version). The future of children ,25,67-87.

Gilbar,O., Plivazky, N., \& Gil, S. (2010). Counterfactual thinking, coping strategies, and coping Resources as predictors of PTSD Diagnosed in physically injured victims of terror attack (Electronic version). Journal of loss and trauma- international perspective on stress \& coping, 15,304-324.

Gilay-Ginor, H. (2015). Infertility counseling. Retrieved March,11,2017, from Hebrew psychology website: http:// www.hebpsy.net/articles.asp.

Ginsburg, E.S. (2016). Procedure for intrauterine insemination (IUI) using processed sperm (Electronic version). UpToDate.

Ginsburg E.S., \& Srouji, S.S. (2016). Donor Insemination (Electronic version). UpToDate. Godkar, O. (2010). New families in psychological perspective. Retrieved August,10,2017, from Hebrew psychology internet website: http:// bebpsy.net/articles.asp? id=2101. 
Goldberg, A.E., Gartrell, N., \& Gates, G. (2014). Research report on LGB parent families. Retrieved August,9,2017, from Williams institute-UCLA internet website: http:// williamsinstitute.law.ucla.edu>lgb-parent-families-July-2014.pdf.

Golombok S. Family Created by Reproductive Donation: Issues and Research- 2012. Society for research in child development, 7, 61-65.

Golombock, S., Zadeh, S., Lmrie, S., Smith V., \& Freeman,T. (2016). Single mothers by choice: Mother- Child relationships and children's psychological adjustment. Journal of family psychology, 30,409-418.

Gonadotropin- releasing hormone antagonist versus GnRH agonist in subfertile couples undergoing assisted-reproductive technology. (2016). Retrieved February,19,2017, from : http:// www.cochranelibrary.com.

Gourounti, K., Anagnostopoulos, F., Potamianos, G., Lykeridou K., Smidt, L., \& Vaslamatzis, G. (2012). Perception of control, coping and psychological stress in infertile women undergoing IVF (Electronic version). Reproductive Biomedicine online, 24,670679.

Graham, S. (2012). Choosing single motherhood? Single women negotiating the nuclear family ideal. In D. Cutas \& S. Chan (Eds), Family beyond the nuclear ideal (1 ${ }^{\text {st }}$ Edition), Chapter 7.

Graneheim, U. H., \& Lundman, B. (2004). Qualitative content analysis in nursing research: Concepts, procedures, and measures to achieve trustworthiness. Nurse Education Today, 24(2), 105-112.

Greenfeld, D., \& Seli, E. (2016). Same-sex reproduction: medical treatment options and psychological considerations. Current opinion obstetrics and gynecology, 28, 202-205.

Haelyon, H. (2010). The psychological needs of women undergoing IVF treatment (Electronic version). Journal of Reproductive Biotechnology and fertility-Sage, 1,20122018.

Hagela, S., Prasad, S., Kumaran, A., \& Kumar, Y. (2016). Stress and infertility: a review. International Journal of Reproduction, Contraception, Obstetrics and Gynecology, 5,940943. 
Harpaz,R. (2010). Coping processes dealing with stress and stressful life events. Retrieved from the web, Jun,6,2017, http:// www.articles.co.il/article/72451.

Hashiloni- Dolev, Y. (2013). The fertility revolution, Moshav Ben-Shemen: Modan Publishing House Ltd.

Heine, S. (2016). The rise of Single Motherhood in the EU: Analysis and Propositions. (Policy paper). Retrieved August,17,2017, from University of Pittsburgh internet website: http:// aei.pitt.edu/id/enprint/74547.

Heyd, D. (2011)."Fruitful and multiply": the basis of the ethics of procreation and genetic shape. Medical law and bioethics, 4,15-36.

Holly, R., Pasch, L., \& Rogers, K. (2015). Risk factors for Anxiety during Lesbian fertility treatment. Fertility and Sterility, 104, 355-356

Hornstein, M., \& Gibbons, W. (2016). Unexplained infertility (Electronic version). UpToDate.

Hudson, K. (2016). Coping complexity model: Coping stressors, coping influencing factors and coping responses (Electronic version). Journal of psychology online, 7, 300-309. Hydrosalpinx. Retrieved January 6,2017, from: http:// ASRM Reproductivefacts.org. In Vitro Fertilization: IVF. Retrieved December 6,2016, from: http:// www.health.gov.il/subjects/fertility/pages/IVF/aspx.

Inhorn, M., \& Patrizio P. (2015). Infertility around the globe: new thinking on gender, reproductive technologies and global movements in the 21 st century. Human reproduction updates, 21,411-426.

Israeli society of obstetrics and gynecology. (2010). Embryo transfer in IVF process. A fact sheets.

IVF guide: retrieved December 9,2016, from http:// www.Assah.org $>$ IVF $>$ Documents.

Kapell, B. (2014). "Mothers by choice, Single by chance": A qualitative exploration of becoming a single mother through the use of medically assisted donor insemination. A thesis for the master's degree, department of sociology, Calgary, Alberta.

Karekla, M., \& Panayiotou, G. (2010). Coping and experiental avoidance: Unique or 
overlapping constructs? Journal of Behavior therapy and experimental psychiatry, 42,163170.

Karniel,S. (2014). Integration of the qualitative and quantitative research in the researcher's toolbox. Heritage, 5, 257-284.

Kouhung, W., \& Hornstein, M.D. (2016). Causes of female infertility (Electronic version). UpToDate.

Kouhung, W., \& Hornstein, M.D. (2016). Evaluation of female infertility (Electronic version). UpToDate.

Krieken van R, Habbis D, Smith P, Hutchins B, Martin G, Maton k. Sociology 5th Edition. Structural change in family life 2014; 116-132

Kuohung, W., \& Hornstein, M.D. (2016). Overview of infertility (Electronic version). UpToDate.

Kuohung, W., \& Hornstein, M.D. (2016). Overview of treatment of female infertility (Electronic version). UpToDate.

Lake, P.P. (2012). Mood symptoms during fertility treatment: the role of hormones. Retrieved from path 2 parenthood website: http:// www.path2parenthood.org.

Lautradis,D. (2016). The possible effect of Hydrosalpinx fluid on Human embryos. Retrieved January,10, 2017 from ESHRE website: http:// www,eshre.eu>surgery>Loutradis.

Lazarus, R., \& Folkman,S. (2008). Stress, Appraisal and Coping (Digital Addition). University of Michigan, Springer publish company.

Lee, S.H., Wang, S.C., Kuo, C.P., Kuo, P.C., Lee, M.S., \& Lee M.C. (2010). Grief responses and coping strategies among infertile women after failed in vitro fertilization treatment. Scandinavian Journal of caring sciences, 24,507-513.

Leibzon,Y. (2017). The state is against adoption by same-sex couples. Retrieved August 13,2017 from Haaretz newspaper website: http:// www.haaretz.co.il.

Levesque, C., Lafontaine, M.F., Caron, A., Flesch, J.L., \& Bjornson, S. (2014). Dyadic Empathy, Dyadic coping, and relationship satisfaction: A Dyadic Model. Europeans Journal of Psychology, 10,118-134.

Liu, K., \& Case, A. (2011). Advanced Reproductive Age and Fertility. SOGC Clinical 
Practice guidelines, 269, 1165-1175.

Lo, W., \& Campo-Engelstein, L. (2018). Expanding the Clinical Definition of Infertility to Include Socially infertile individuals and couples. Reproductive Ethics (Electronic Version) 71-83.

Lofquist,D. (2011). Same-sex householders. Retrieved August,5,2017, from Census Bureau internet website: http:// www.census.gov>assbr 10-03.

Lopes, V., Canavarro, M.C., Verhaak, C.M, Boivin, J., \& Gameiro,S. (2014). Are patients at risk of psychological maladjustment during fertility treatment less willing to comply with treatment? Results from the Portuguese validation of the SCREENIVF. Human Reproduction, 29,293-302.

Mahlstedt ,P.P. (2009). Coping with infertility: How family and friends can help. Retrieved June,28,2017, from the national infertility association internet site: http: // WWW.resolve.org.

Mahmoud, M.K., Punukollu, D \& Mahmood, T. (2013). In Vitro Fertilization: Obstetrics, Gynecology and Reproductive Medicine, 23, 238-246.

Mahmoudi, R., Mortazvi, S.M.J., Safari, S., Nikseresht, M., Mozdaran, H., Jafari, M., Zamar, A., Hagani, M., Davari, M., Tabatabai, A., \& Solemani ,A. (2015). Effects of microwave electromagnetic radiations emitted from common Wi-Fi routers on rat's sperm count and motility. International Journal of Radiation Research, 13,363-368.

Martins, M.V., Basto-Pereira, M., Pedro, J., Peterson, B., Almeida, V., Schmidt, L., \& Costa, M.E. (2016). Male psychological adaption to unsuccessful medically assisted reproduction treatments: a systematic review. Human Reproduction Update, 22,466-478.

Martins, M.V., Peterson, B.D., Almeida, V.M., \& Costa, M.E. (2011). Direct and indirect effects of perceived social support on women's infertility-related stress. Human Reproduction, 8,2113-2121.

Martins M.V., Peterson B.D., Almeida V., Mesquita-Guimarães J., Costa M.E. (2014) Dyadic dynamics of perceived social support in couples facing infertility. Hum. Reprod. 29:83-89.

McLeod, S. (2015). Stress management. Retrieved, May,7,2017, from Simply psychology internet site: http://www.simplypsychology.org/stress-management.html. 
Mencaglia, L., Albuquetqe- Neto, L., \& Arias-Alvarez, R.A. (2013). Manual of Hysteroscopy: Diagnostic, operative and office Hysteroscopy. Retrieved November,30,2016, from: http:// websurg.com>get>pdf.

Mikkelson, A.T., Madsen, S.A., \& Humadian, P. (2012). Psychological aspects of male fertility treatment (Electronic version). Journal of Advanced Nursing, 69,1977-1986.

Moore,T.J., Asay, S.M. (2012). Understanding Families (Sage Publications). In family resource management (Chapter 2, pp. 25-43).

Neubauer, B., Witkop, T.C., \& Varpio, L. (2019). How phenomenology can help us learn from the experiences of others. Perspectives on Medical Education ,8, 90-97.

Operative Hysteroscopy. Retrieved November,25,2017, from Goldshmidth,H- MD- Director of Gynecological Endoscopy unit. (Rabin center). Internet site: http: $\underline{\text { www.hysteroscopy.co.il }}$

Orvieto, R., \& Patrizio, P. (2013). GnRH agonist versus GnRH antagonist in ovarian stimulation: an ongoing debate. Reproductive Biomedicine online, 26,4-8.

Parmegiani, L., Cognigni, G., \& Filicori, M. (2012). New advances in Intracytoplasmic Sperm Injection (ICSI). Retrieved from the internet March 15,2017, from http:// www.intechopen.com.

Parsons, T., \& Bales, R.F. (2007). The American Family: its relations to personality and to the social structure. In T\& F, Routledge (Eds), Family: Socialization and interaction process (Electronic addition). Great Britain.

Patton, M. Q. (Ed.). (2002). Qualitative Research \& Evaluation Methods (3rd ed.). Thousand Oaks, CA: Sage.

Paulson, R. (2016). In Vitro Fertilization (Electronic version). UpToDate.

Pelkonen, S. (2016). Frozen Embryo Transfer: Early pregnancy, Perinatal outcome, and health of singleton children. Retrieved from the internet March,21,2017, from: http:// www.Julkita.oulu.fi.

Peterson, B.D., Pirritano, M., Block, J.M., \& Shmidt, L. (2011). Marital benefit and coping strategies in men and women undergoing unsuccessful fertility treatments over 5-year period. Fertility and Sterility, 95,1759-1763. 
Peterson, B.D., Sejbaek, C.S., Pirritano, M, \& Shmidt, L. (2014). Are severe depressive symptoms associated with fertility-related distress in individuals and their partners? Human reproduction, 29 ,76-82.

Ponto, J. (2015). Understanding and Evaluating Survey Research. Journal of the advanced Practitioner in Oncology, 6(2):168-171.

Post-coital test. Retrieved November,8,2016, from: http:// Medical Forms/fertility/postcoital test/12-7-7.

Practice Committee of the American Society for Reproductive Medicine. (2016). Prevention and treatment of moderate and severe ovarian hyperstimulation syndrome: a guideline. Fertility and Sterility, 106,1634-1635.

Quant, H.S., Zapantis, A., Nishen, M., Beviliacqua, K., Jindal, S., \& and Pal, L. (2013). Reproductive implications of psychological distress for couples undergoing IVF (Electronic version). Springer, 30,1451-1458.

Rabbi Haeitan Elijah. (2013). Embryos reduction. Retrieved April 3,2017 from Puah institute internet site: http://www.puah.org.il>page.

Ray, P., Shah, A., Gudi, A., \& Homberg, R. (2012). Unexplained infertility: an update and review of practice. Reproductive BioMedicine online, 24,591-602.

Rights of single mothers (2008). Retrieved August,10,2017, from National Insurance Institute of Israel's internet site: http://www.btl.gov.il.

Rodin, S., Almog, O, \& Paz, D. (2008). Same-sex families in Israel. Retrieved September,3,2017, from the Guide to Israeli society's internet site: http://www.peopleil.org/details.axp?itemID=7543.

Schwerdtfeger, K.L \& Shreffler, K.M (2009). Trauma of pregnancy loss and infertility among mothers and involuntarily childless women in the United States. PubMed ;14(3):211-227.

Scheib.J.E., \& Hastings,P.D. (2012). Donor-Conceived children raised by Lesbian couples : Socialization and development in a new form of planned family. Retrieved July 25,2017, from parenting network's internet site: http://www.thespermbankofca.org/files/2012ScheibHastingsjurnal.

Shechner, T. (2013). Relations between social support and psychological and parental distress 
for lesbian, single heterosexual by choice and two-parent heterosexual mothers. (Electronic version). PubMed,80,283-292.

Shrestha, D., La, X., Feng, H.L. (2015). Comparison of different stimulation protocols used in In Vitro Fertilization: a review. Annals of translational Medicine, 3,1-7.

Sick days for fertility treatments. Retrieved December,14,2016 from: http://www.kolzchut.org.il.

Simonstein, F., Eizenberg-Mashiach, M., Revel, A., \&Younis, J.S. (2014). Assisted reproduction policies in Israel: a prospective analysis of in vitro fertilization- embryo transfer. Fertility and Sterility,102,1301-1306.

Sincero, S. (2012). Stress and cognitive appraisal. Retrieved May,6,2017, from Explorable internet site: http://explorable.com.

Shkedi, A. (2003). Words trying to touch: Qualitative Research- Theory and Application. 4, 69-80. Tel-Aviv- Ramot.

Song, S.H, Kim, D.S., Yoon, T.K., Hong, J.Y., \& Shim, S.H. (2016). Sexual function and stress level of male partners of infertile couples during the fertile period. BJU international, $117,173-176$.

Sperling, D. (2010). Commanding the "Be Fruitful and Multiply" directive: Reproductive Ethics, Law, and policy in Israel. Cambridge Quarterly of Healthcare Ethics, 19,363-371.

Stimpfel, M., Vrtacknik-Bokal, E., Pozlep,B.,\& Virant-Klun,I. (2015). Comparison of GnRH agonist, GnRH antagonist, and GnRH antagonist mild protocol of controlled ovarian hyperstimulation in good prognosis patients. International Journal of Endocrinology, 15,16

Stryar R. (2009). Social involvement edition, Society and welfare ,2.213-232.

Swerdloff, R.S., \& Wang, C. (2016). Causes of male infertility (Electronic version). UpToDate.

Swerdloff, R.S., \& Wang, C. (2016). Evaluation of male infertility (Electronic version). UpToDate.

Taylor,S.J.,\& Bogdan,R. (1984). Introduction to Qualitative Research Methods: The search for meanings, 2nd ed. New York: John Wiley. 
Termination of pregnancies by law. 1990-2013. Retrieved September 2,2017 from the Ministry of health website: http:// www.health.gov.il.

The ethical approach to gamete donations anonymity (2015). Retrieved October,15,2016.

From ethics committee's attitude of the Israeli Fertility Association (IFA) website: http://www.ayala.org.il>viewcontent.

Practice Committee of the American Society for Reproductive Medicine (2014). - Guidance on the number of embryos transfer. ASRM / SART, Birmingham. Alabama.

Toftager M., Bogstad J., Bryndorf T., Lossl K., Roskaer J., Holland, T., Preatorius L., Zedeler, A., Nilas L., \& Pinborg ,A.(2016). Risk of severe ovarian hyperstimulation Syndrome in GnRH antagonist versus GnRH agonist protocol: RCT including 1050 first IVF/ICSI cycles. Human Reproductive, 31,1253-1264.

The state is against adoption by same-sex couples. Retrieved July,16,2017 from Health news website: http://haaretz.co.il.

Tsai, H.C., Kuo, T.N., Chung, M.T., Lin M.Y.S., Kang, C.Y., \& Tsai Y.C. (2015). Acute abdomen in early pregnancy due to ovarian torsion following successful In VitroFertilization treatment. Taiwanese Journal of obstetrics \&Gynecology, 54, 438-441.

Vanorman, A.G., \& Schommegna, P. (2016). Understanding the dynamics of family change in the USA. Population Reference Bureau, Population bulletin Journal, 71, 2-14.

Verheyen, G. (2013). Practical management of IVF patients with TESE/ TESE/ PESA: Fresh and frozen samples. Retrieved December,18,2016 from Global Fertility Academy website: http://www.globalfertilityacademy.org.

Viner-Uman, A. (2012). Recommendations of the public committee to examine legislative series of fertility and birth in Israel. Retrieved August,21,2017 From Ministry of health website: http://www.health.gov.il.

Wang, C., \& Swerdloff, R.S. (2016). Treatment of male infertility (Electronic version). UpToDate.

Wischmann, T. (2013). "Your count is zero"- counseling the infertile man. (Electronic version) Pubmed-Human fertility 1-5.

Yenontan,L. (2016). The government regulates the sperm donation: a man can donate to nine 
women at the most. Retrieved November,5,2016 from Health news website:

http://haaretz.co.il.

Yaun, A., Zhuangzhuang, S., Linan, L., Yajuan, Z., \& Hongping, J. (2013). Relationship between psychological stress and reproductive outcome in women undergoing in vitro fertilization treatment: Psychological and neuro-hormonal assessment (Electronic version). Springer,30,35-41.

Yilmez, T., \& Oskay, U. (2016). The Copenhagen Multi-Centre Psychological Infertility (COMPI) fertility problem stress and coping strategy scales: A Psychometric Validation Study in Turkish fertile couples. International journal of caring science,9, 458-459.

Zelkowitz, P., Carrier, M.E., Whitley, R., Tulandi, T., \& Ells.C. (2003). Too involved, or not involved enough? Infertile couples' perceptions of social support. Fertility and Sterility, 104,355-356. 


\section{Chapter 8: $\underline{\text { Annexes }}$}

\section{Research questionnaire}

Dear participant, current questionnaire is part of a doctoral thesis dealing in support systems during fertility treatments. It is important to indicate that honest answers are important for success of the study. I would appreciate should you agree to participate in the study.

I would like to emphasize that the questionnaire is completely anonymous, its findings are confidential and intended for research purposes of current study only.

Thank you for your invaluable time and cooperation.

Limor Rimer

General questions:

1. Age:

2. Education:

- High school

- Diploma

- Academic degree- BA

- Second degree and above

3. Marital status:

- Married

- Divorced

- Bacheloratte

- Partnership

- Female spouse

Details of spouse/ female spouse

1. Age:

2. Education:

- High school

- Diploma

- Academic degree-BA

- Second degree and above

3. Years of marriage / cohabitation:

4. Number of children: 
5. How long have you been trying to conceive?

6. Tell me - what have you done since?

7. Elaborate if you can, which examinations have you been through?

8. Elaborate if you can, which treatments have you been through?

\section{$\underline{\text { Support }}$}

1. In what way does your close family support you?

2. How does your partner support you?

3. Have you and how were you assisted by support outside the family frame psychologist, social worker, support groups etc.

4. Describe your feelings during the treatments 
5. Have you and how do you share your feelings with the close environment and your partner? Give details.

\section{$\underline{\text { Partner and family }}$}

1. To what extent is the partner being an active partner to examinations, treatments and the days after?

2. How is the intimacy relationship with your partner being affected by the treatments?

3. How do you cope (as a couple) with unsuccessful fertility treatments?

4. What is the support system that you gain from your family members, to an unsuccessful treatment? Give details.

\section{Coping}

1. How do you overcome an unsuccessful treatment?

2. What do you do in case of an unsuccessful treatment? 
3. Where there moments when you blamed yourself or your partner for the failure of the treatment?

4. To what extent family / friend's get-togethers affect you?

5. Elaborate if you can, to what extent the treatments have affected your routine

6. To what extent do you believe that this is a matter of fate?

7. Have you and how do you accept the situation

8. Have you considered giving up? In which stage? 


\section{Coded questionnaire}

Dear participant, current questionnaire is part of a doctoral thesis dealing in support systems during fertility treatments. It is important to indicate that honest answers are important for success of the study. I would appreciate should you agree to participate in the study.

I would like to emphasize that the questionnaire is completely anonymous, its findings are confidential and intended for research purposes of current study only.

Thank you for your invaluable time and cooperation.

Limor Rimer

\section{General questions:}

1. Age:

2. Education:

- High school

- Diploma

- Academic degree- BA

- Second degree and above

3. Marital status:

- Married

- Divorced

- Bacheloratte

- Partnership

- Female spouse

Details of spouse/ female spouse

1. Age:

2. Education:

- High school

- Diploma

- Academic degree-BA

- Second degree and above

3. Years of marriage / cohabitation:

4. Number of children:

5. How long have you been trying to conceive? 
1. Less than a year

2. 1-2 years

3. 2-3 years

4. 3-4 years

5. 4-5 years

6. Over 5 years

6. Tell me - what have you done since?

1. Went to a fertility specialist

2. Various of fertility tests

3. Fertility treatments / IVF.

4. Alternative medicine (Acupuncture, Reflexology, act.)

7. Elaborate if you can, which examinations have you been through?

1. PAP smear

2. Hysterosalpingography

3. Hysteroscopy

4. Blood tests (Hypercoagulation, AMH, Karyotype, hormonal profile).

5. Ultrasound- follow up

6. Breast test / Mammography

7. Electrocardiogram

8. Elaborate if you can, which treatments have you been through?

1. IVF treatments / Embryo transfer

2. Clomid + IUI

3. Hormonal treatment (Gonal-f, Puregon, Menopur)

\section{$\underline{\text { Support }}$}

1. In what way does your close family support you?

1. Full support

2. Partial support

3. Try to cheer up / help

4. Aware of difficulty, but not helping 
5. Do not support at all

6. Not involved at all

2. How does your partner / Female partner support you?

1. Very supportive

2. Partial support (make an effort).

3. Barely support

4. Not supportive at all

5. Irrelevant (for single women)

3. Have you and how were you assisted by support outside the family frame - psychologist, social worker, support groups etc.

1. Did not need a support

2. Psychologist / social worker

3. Support groups (Facebook, internet, watsup, of the HMO

4. Alternative treatment.

4. Describe your feelings during the treatments

1. Stress / distress

2. Frustration / despair / helplessness

3. Disappointment / failure / lack of optimism

4. Fear / fright / uncertainty

5. Anger / envy / irritability / impatience

6. Difficulty / pain

7. Guilt / self-blame

8. Loneliness

9. Lack of self-control / emotional turmoil

10. Mood swings (hope- despair)

11. Crying / sadness / depression.

12. Hope / Faith/ optimism / expectation

5. Have you and how do you share your feelings with the close environment and your partner? Give details.

1. Do not share anything

2. Not sharing everything

3. Only my spouse / female spouse

4. Only a family member

5. Close friends

6. Talking about it / sharing it all

\section{$\underline{\text { Partner and family }}$}

1. To what extent is the partner being an active partner to examinations, treatments and the days after?

1. Full partner 
2. Partially partner

3. Very little / almost no

4. Not a partner at all

5. irrelevant (for single women)

2. How is the intimacy relationship with your partner being affected by the treatments?

1 . Was adversely affected

2. Was somewhat affected

3. Was hardly affected

4. Was not affected at all

5. On the contrary, the treatments strengthen our relationship

6. Irrelevant (for single women).

3. How do you cope (as a couple) with unsuccessful fertility treatments?

1. Crying / pain

2. Disappointment / frustration

3. Optimism / strengthening

4. Conversations / hearkening

5. Expectation / hope / faith

6. Each one copes in his own way

7. Irrelevant (for single women).

4. What is the support system that you gain from your family members, to an unsuccessful treatment? Give details.

1. Encouragement / Empathy

2. Hearkening / conversations

3. Giving advice

4. Strengthening

5. No support / not involved.

\section{Coping}

1. How do you overcome an unsuccessful treatment?

1. Crying / Sadness / disappointment.

2. Drink Alcohol / eat / smoke.

3. Going for shopping / movie

4. Optimistic / moving on

5. Meditation / conversations

6. Occupy me with work duties / routine.

7. Cannot overcome / difficulty in overcoming

2. What do you do in case of an unsuccessful treatment?

1. Crying / falling apart

2. I withdraw into myself / shut myself up / sleep.

3. Moving on to the next treatment.

4. going out / for a vacation.

5. Smoke, drink Alcohol / eat. 
6. Share, talk about the situation.

7. Remain optimistic.

8. Continue a normal daily routine.

9. Pause, timeout.

3. Where there moments when you blamed yourself or your partner for the failure of the treatment?

1. Not at all.

2. Blamed me

3. Blamed my spouse

4. I blamed both of us.

4. To what extent family / friend's get-togethers affect you?

1. Do not affect at all.

2. Low impact (slightly go)

3. Very much (avoided going).

4. Help, a positive effect.

5. Elaborate if you can, to what extent the treatments have affected your routine

1. Had no effect at all.

2. Not so influential.

3. Influence on a certain extent.

4. Constitute a large part of the daily routine.

5. Had a significant effect (considerably).

6. To what extent do you believe that this is a matter of fate?

1. I don't believe at all

2. I don't know / it's hard to tell.

3. So-so

4. Quite believe.

5. Believe strongly. 
7. Have you and how do you accept the situation

1. Did not accept (continue trying)

2. Haven't accepted yet.

3. Start to accept.

4. I accept the situation.

5. Not willing to accept / hard to accept.

8. Have you considered giving up? In which stage?

1. Not at all.

2. thought about it, took a pause.

3. After a certain number of treatments.

4. Almost after every failed treatment.

5. Considering now. 


\section{Interview Questions:}

1. Tell me about yourself if you please (age, marital status, residence, education level, workplace, years of marriage / cohabitating and number of children).

2. Please tell me about your current life and your desire for a child. (how long have you been trying to conceive? What drove you to turn to medical assistance?)

3. Tell me about your treatment process you are going through to achieve this desire (what kind of treatments you have undergone?)

4. Tell me about the difficulties you encountered during the process

5. How did you manage with the difficulty in general and with treatments in particular?

6. what was your family, society, your spouse / female spouse, workplace and friend's reaction through the whole process? What about the medical system, where there any obstacles during the process?

7. Can you describe your feelings before and during the treatments? (what kind of thoughts you had in mind before and during the treatment)

8. Tell me what kind of support and assistance you have received, like: psychologist, social worker and support groups.

9. Where there moments when you decided to take a recess of treatments, or give up?

Additional questions for married interviewees and with female spouse:

10. Tell me about the support you have received from your spouse / female spouse

11. How did you manage the treatments together as a couple? 
Informed consent

\section{I the undersigned:}

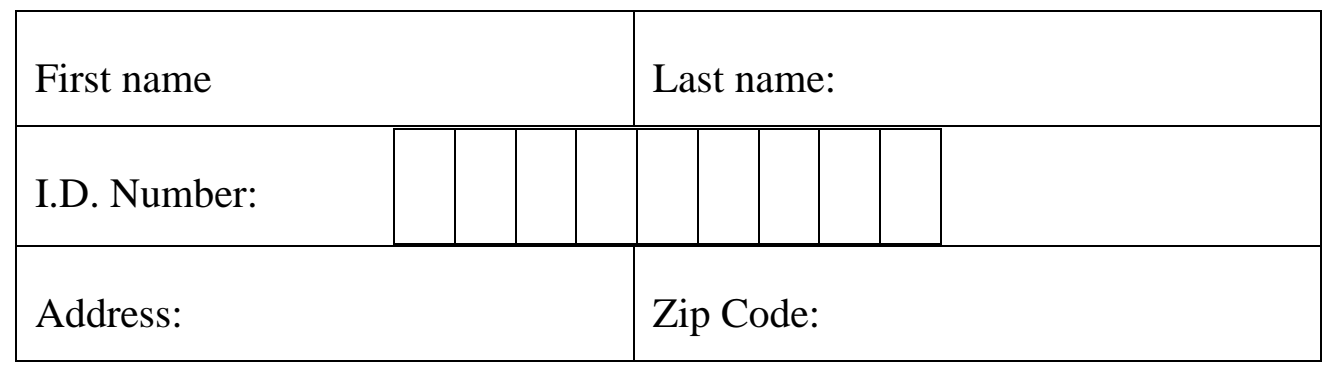

1.) Hereby declare that I give my consent to participate in medical experiment, as specified in this document.

2.) I declare that I was explained by:

Name of the explaining researcher:

2.1) That the main researcher (name of physician): Mrs. Limor Rimer received from director of the medical institution (Maccabi), an approval to conduct the study, according to the meaning thereof in The People's Health Regulations (Human Medical Experiments, 1980).

2.2) That the medical experiment is conducted on the subject: Impact of familial structure and support on women's coping with fertility treatments.

2.3) That I am free to choose not to participate in the medical experiment, and that I am free to end my participation in the experiment, at any time, and all that with no damaging my right to receive the common treatment

2.4) That in case of filling a questionnaire - I have the right not to answer all the questions in the questionnaire or part of them.

2.5) That it is promised to me that my personal identity will be kept secret by all those dealing and involved in the study and will not be published in any publication, including scientific publications.

3) I declare that I was given a detailed information regarding the medical experiment according to following specified subjects: 
3.1) General background and research importance. Fertility conceal within them multiple emotional aspects and they affect and have implications on other fields of life such as intimacy and social relations, workplace relations, sense of personal welfare and general quality of life.

3.2) Research purpose. The study will examine the way women undergoing fertility treatments cope and the support systems available to them within the family frame.

3.3) What is required from a participant in frame of the study. Filling an anonymous questionnaire after detailed explanation regarding the essence of the study from the lead researcher and expressing an informed consent.

3.4) Expected period of the length of time of participation in the study. 8-12 months.

3.5) Expected benefits for the participant or for others, as a result of the study. The research data will allow the understanding of the difficulties that women experience during fertility treatments. Additionally, we will try to evaluate whether there are sufficient support systems for these women and how can we enlist for the benefit of the process they undergo.

3.6) The discomfort that might arise as a result of participation in the study. The questionnaire includes inquisitive questions and responding might cause discomfort.

\section{7) Current study does not include data collection from a pool.}

3.8) Another relevant information (as given by initiator of the experiment). Should a need arise during the interview - the patient will be referred according to the recommended treatment.

4) I hereby declare that this consent is given with my free will and that I understood all the above. Additionally, I received a copy of this informed consent form, carrying a date and legally signed.

5) With my signature on this consent form, I allow the initiator of the medical experiment (through the lead researcher) access to my medical file, and as well to the institutional Helsinki Committee, the comptroller entity in the medical institution and the Ministry of Health a direct access to my medical file, for purpose of verification of the medical experiment methods and the clinical data. This access to my medical information will be done with confidentiality, according to confidentiality laws and regulations. 


\begin{tabular}{|c|c|c|}
\hline $\begin{array}{c}\text { Name of participant in the } \\
\text { medical experiment }\end{array}$ & $\begin{array}{c}\text { Signature of participant in } \\
\text { the experiment }\end{array}$ & date \\
\hline & & \\
\hline
\end{tabular}

Declare of the researcher / secondary researcher:

Following consent was received by me, that is after explaining the participant in the medical experiment all the above and I as well made sure that all my explanations were comprehended by them.

\begin{tabular}{|c|c|l|}
\hline $\begin{array}{c}\text { Name of the explaining } \\
\text { researcher }\end{array}$ & $\begin{array}{c}\text { Signature, stamp, and } \\
\text { license number }\end{array}$ & Date \\
\hline & & \\
\hline
\end{tabular}




\section{Research approval}

\section{Form 17}

Approval of the institution's director for research on existing data and questionnaires

Date: 27-May-2018

For Mrs Limor Rimer, Womens haelth care

Maccabi health services

Under discussion: approval for conducting research on existing data and questionnaires.

in accordance with your request dated $05 / \mathrm{May} / 2018$, it is hereby approved to conduct the srudy according to the application documents.

\section{Study details}

\section{Institutional application number}

0127-17-BBL

\section{The study's subject}

The effect of family structure and family support on women coping with fertility treatments

Initiator's name and its address

Mrs. Limor Rimer

Initiator's representative in Israel and its

Multi-central study in the country-yes address

\section{Study's documents}

\begin{tabular}{|l|l|l|}
\hline $\begin{array}{l}\text { Study's protocol- name/number } \\
\text { 0127-17-BBL }\end{array}$ & Version & $\begin{array}{l}\text { Date } \\
27 / \mathrm{March} / 2018\end{array}$ \\
\hline $\begin{array}{l}\text { Informed consent-name/number } \\
\text { Form 2 Hebrew }\end{array}$ & Version & Date \\
& 2 & $27 / \mathrm{March} / 2018$ \\
\hline
\end{tabular}

By virtue of the consent I received by the Ministry of health's general director, to approve as "director" for conducting study on existing data and questionnaires, in the medical institution , after the application was approved by institutional Helsinki's subcommittee on :23/may/2018 and after being convinced that the study is in accordance with the declarations of Helsinki's principles and human experiment procedure, I hereby approve the conduction of the study according to the following conditions: 


\section{Form 17}

Approval of the institution's director for research on existing data and questionnaires

\section{The approval's conditions}

1. The research will be conducted in accordance with the Helsinki declaration's principles and in accordance with the procedure's requirements for medical experiments in humans in Israel (procedure 14,2016). And the up to date international procedure's requirements.

2. The research will be conducted only after providing the participant or his legal representative an explanation and signing an inform consent form attached to the request (in appropriate cases).

3. Any changes, additions or deviations from the research plan, require the medical institution's Helsinki sub-committee's A written approval.

4. The main study researcher must provide to the Helsinki subcommittee a concluding report on the research's process and its results.

5. Extending the medical research's validity: three months before passing the approved period of the medical research. The main investigator must report the Helsinki subcommittee of the medical institution about the research process's progress. The committee will announce its decision regarding the research's continuation to the medical institution's director. The director will issue a new research's certificate.

6. At the end of the research's period, the main researcher will submit the Helsinki committee a summary report on the research' process and its results.

7. The approval is granted only to the main researcher and the medical institution indicated above and it is untransferable.

8. No information about the medical research should be published in the mass media, such as journalism, radio, television, the internet, except for publication in scientific journals or scientific conferences, and except for recruitment of the research's participants advertising.

9. Document's retention: all application documents, approvals and all documents collected during the medical research must be kept for at least seven years from the end of the research.

10. Additional restrictions: intermediate report is required after 180 days

The research is approved for 450 participants only

11. Approval validity: one year from the signing date. 
Sincerely

Director of the medical Institution

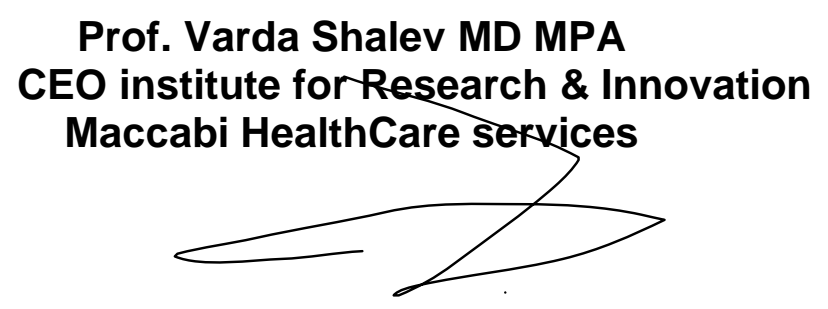

Copy: Chairman of the Helsinki committee Dr. Sasson Gizel -Ayala

Research initiator / his representative in the country (through the researcher) Mrs. Limor Rimer.

Department of clinical trials, department of pharmacy - Ministry of health 